\title{
Modulation of cardiac function by oxidized type I protein kinase $\mathrm{A}$
}

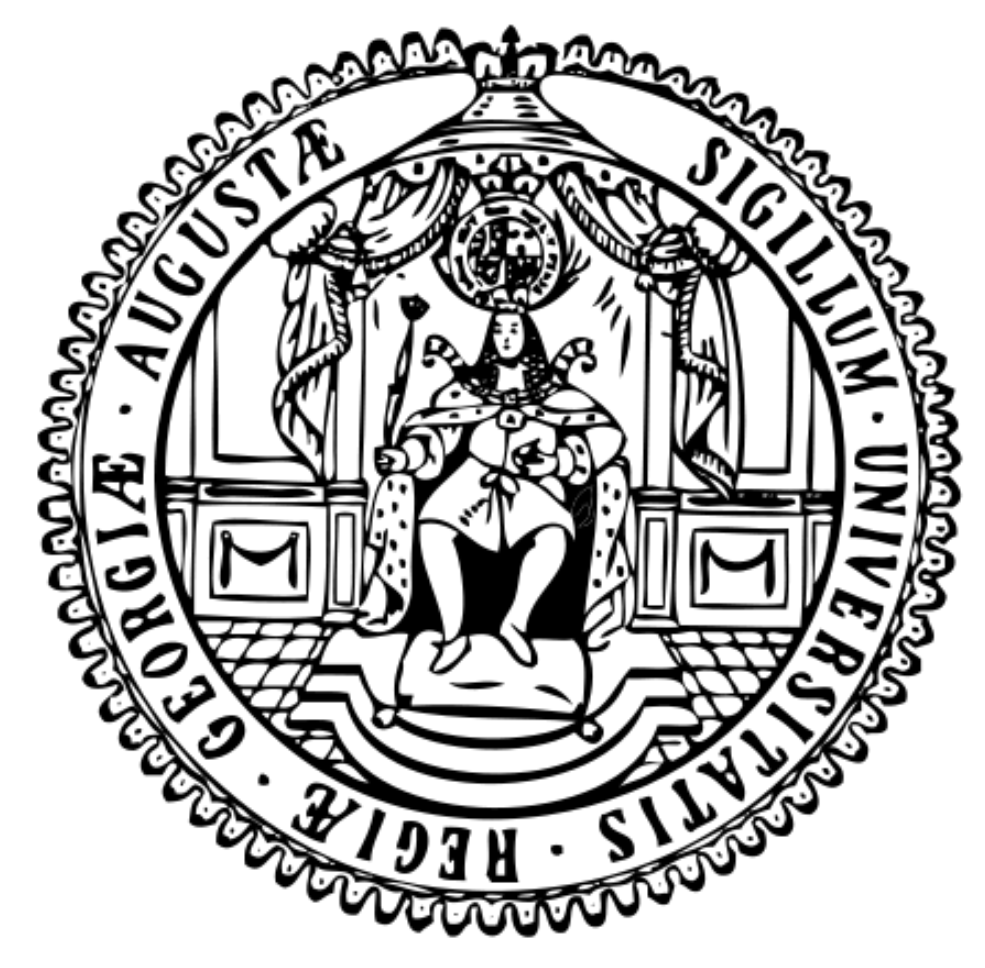

Doctoral Thesis

In partial fulfillment of the requirements for the degree "Doctor of Philosophy (PhD)" in the Molecular Medicine Study Program at the Georg-August University Göttingen.

Submitted by

M M Towhidul Islam

(Born in Dhaka, Bangladesh)

Göttingen, 2016 


\section{Declaration}

Here I state that the doctoral thesis entitled "Modulation of cardiac function by oxidized type I protein kinase A" has been written independently with no other sources and aids than quoted. Furthermore, this thesis has not been submitted completely or partially for another examination process neither in identical nor in similar form.

M M Towhidul Islam

March 2016 


\section{Committee Members}

\section{Prof. Dr. med. Lars S. Maier}

Supervisor

Director, Clinic and Polyclinic for Inner Medicine II, University Medical Regensburg and Department of Cardiology and Pneumology, University Medical Göttingen

Lars.Maier@klinik.uni-regensburg.de

\section{Prof. Dr. med. Stefan Wagner}

\section{Supervisor}

Clinic and Polyclinic for Inner Medicine II, University Medical Regensburg and Department of Cardiology and Pneumology, University Medical Göttingen

Stefan.Wagner@klinik.uni-regensburg.de

\section{Prof. Dr. Philip Eaton}

Supervisor

Department of Cardiovascular Biochemistry, King's College London

Philip.eaton@kcl.ac.uk

\section{Prof. Dr. Wolfram-Hubertus Zimmermann}

Thesis committee member

Director, Department of Pharmacology and Toxicology, University Medical Göttingen

W.zimmermann@med.uni-goettingen.de

\section{Prof. Dr. mult. Thomas Meyer}

Thesis committee member

Department of Molecular Psycho-cardiology, University Medical Göttingen

Thomas.meyer@med.uni-goettingen.de

\section{Prof. Dr. Dörthe M. Katschinski}

Extended thesis committee member

Director, Department of Cardiovascular Physiology, University Medical Göttingen

Doerthe.katschinski@med.uni-goettingen.de

\section{Prof. Dr. Michael Zeisberg}

Extended thesis committee member

Clinic for Nephrology and Rheumatology, University Medical Göttingen

Mzeisberg@med.uni-goettingen.de 


\section{List of publications}

NADPH oxidase 2 mediates angiotensin II-dependent cellular arrhythmias via PKA and CaMKII. (2014) Stefan Wagner, Christian Dantz, Hannah Flebbe, Azadeh Azizian, Can Martin Sag, Susanne Engels, Johanna Möllencamp, Nataliya Dybkova, Towhidul Islam, Ajay M. Shah, Lars S. Maier. Journal of Molecular and Cellular Cardiology, 75, 206-215.

Modulation of Cardiac Function by oxidized PKA. T. Islam, Dantz C, Daniel H, Moellencamp H, Armouch AE, Eaton P, Maier L, Wagner S. (manuscript under preparation)

CASK is an important regulator of cardiac excitation-contraction coupling. J. Mustroph, S. Gupta, A. Dietz, F. Bähr, T. Islam, A. El-Armouche, L.S. Maier, S. Wagner. (manuscript under preparation)

Sleep-disordered breathing is associated with increased CaMKII-dependent SR $\mathrm{Ca}^{2+}$ leak in human Atrial Cardiomyocytes. M.A. Drzymalski, S. Ripfel, S. Meindl, A. Biedermann, M. Durczok, T. Islam, S. Katz, M. Mendl, T. Weizenegger, B. Flörchinger, D. Camboni, S. Wittmann, J. Backs, C. Schmid, L.S. Maier, M. Arzt, S. Wagner. (manuscript under preparation)

Relationship between postmenopausal obesity and CaMKII via p38MAP kinase pathway in the atrium. T. Tsuneda, S. Neef, T. Islam, L. S. Maier (manuscript under preparation) 


\section{Acknowledgements}

Firstly, I would like to express my heartfelt gratitude to my supervisors Prof. Lars Maier, Prof. Stefan Wagner and Prof. Philip Eaton for their continuous support during my Ph.D. study. Lars, you have been a tremendous mentor for me. I always follow you as my idol. Phil, thanks for encouraging me on redox research and steered me to grow as a research scientist. A very special thanks to Stefan for his patience, motivation, and guidance throughout my PhD. Whenever I talked to you, I have learned something new. Your advice on both researches as well as on my career has been priceless. It was a big opportunity for me to have such cooperative and well-reputed mentors with me. I conveyed my deepest gratitude to Lars and Stefan for selecting me and gave me that opportunity.

Moreover, I would like to sincerely thank Prof. Zimmermann and Prof. Mayer for their insightful comments and encouragement, which incented me to widen my research from various perspectives. My special appreciation is for Prof. Katschinski for her caring and ever smiley attitude and for being with me in both good and bad time of my PhD. I should also mention Prof. Ali and Prof. Nikolaev for their endless inspiration. Thanks also go to Prof. Schäfer and Prof. Elisabeth for their cooperation. I am very grateful to Prof. Sossalla and Dr. Fischer as well for their immense support during last three years.

My deepest thanks go to Nataliya, Takayuki, Nico, Rajni, Xinbo in Göttingen and to Burgoyne, Ewald, Olena in London who provided me the opportunities to work with them. Moreover, many thanks to Anna, Angelika, Jonas, Shamin, Theresa, Jing and Satish for their cooperation in Göttingen. In addition, thanks to Asvi, Celine and Yemi who helped me a lot during my stay in London. I also thank to all my other lab mates both in Göttingen and Kings College, for all the fun we have had in the last three years, without their valuable support it would not be possible to conduct this research. I should also mention all my friends and country mates here in Göttingen with whom I feel extremely homely here.

I would especially like to thank all the expert hands from Timo, Thomas, Kim, Sarah, Sabrina, Marcel, Roland, Beate and Kirsten during the experiments. Also thanks to Brockmann, Daniela, Ilka, Jutta and Nina for their immeasurable help in the animal facility. Without all of their passionate participation and input, experiments could not have been completed.

I finish with Bangladesh, where the most basic source of my life energy resides: my family. I have an amazing family, unique in many ways, and the stereotype of a perfect family in many other ways. I would like to thank my parents and to my brother and sisters for supporting me spiritually throughout my life and also during this PhD. Heartfelt thanks to my wife Tajnin, who never let me feel alone especially during my tough time and my son Tahmeed, who makes me smile. Tajnin has cherished with me every great moment and supported me whenever I needed it. All of her support has been unconditional for all these years.

I am really grateful to all of you. 


\section{List of abbreviations}

\begin{tabular}{|c|c|}
\hline A & Ampire \\
\hline $\mathrm{AC}$ & Adenylyl cyclase \\
\hline ACE & Angiotensin-converting enzyme \\
\hline $\mathrm{AF}$ & Atrial fibrillation \\
\hline AHI & Apnea-hypopnea index \\
\hline AKAP & A-kinase anchor proteins \\
\hline $\mathrm{AM}$ & Acetoxymethyl ester \\
\hline AMP & Adenosine monophosphate \\
\hline AngII & Angiotensin II \\
\hline $\mathrm{AP}$ & Action potential \\
\hline APD & Action-potential duration \\
\hline APS & Ammonium persulfate \\
\hline ATP & Adenosine triphosphate \\
\hline AV & Atrio-ventricular \\
\hline AWThd & Anterior wall thickness in diastole \\
\hline BCA & Bovine calf serum \\
\hline BDM & 2,3-butanedione monoxime \\
\hline Bp & Base pair \\
\hline bpm & Beats per minute \\
\hline BSA & Bovine serum albumin \\
\hline BW & Body weight \\
\hline $\mathrm{Ca}^{2+}$ & Calcium \\
\hline $\mathrm{CaM}$ & Calmodulin \\
\hline CaMKII & $\mathrm{Ca}^{2+} /$ calmodulin-dependent protein kinase II \\
\hline cAMP & 3',5'-cyclic adenosine monophosphate \\
\hline $\mathrm{CaSpF}$ & $\mathrm{Ca}^{2+}$ spark frequency \\
\hline $\mathrm{CI}$ & Cardiac index \\
\hline CICR & Calcium-induced calcium release \\
\hline $\mathrm{Cm}$ & Membrane capacitance \\
\hline $\mathrm{CO}$ & Cardiac output \\
\hline CVD & Cardiovascular disease \\
\hline DAD & Delayed afterdepolarization \\
\hline $\mathrm{dd} \mathrm{H}_{2} \mathrm{O}$ & Double distilled water \\
\hline DMSO & Dimethyl sulfoxide \\
\hline DNA & Deoxyribonucleic acid \\
\hline dNTP & Deoxyribonucleoside triphosphate \\
\hline DTT & Dithiothreitol \\
\hline EAD & Early afterdepolarization \\
\hline ECC & Excitation-contraction coupling \\
\hline ECG & Electrocardiogram \\
\hline Echo & Echocardiography \\
\hline EDTA & Ethylenediaminetetraacetic acid \\
\hline $\mathrm{EF}$ & Ejection fraction \\
\hline EGTA & Ethylene glycol tetraacetic acid \\
\hline
\end{tabular}




\begin{tabular}{|c|c|}
\hline EPU & Electrical programmed stimulation \\
\hline ER & Early repolarization \\
\hline ETC & Electron transport chain \\
\hline $\mathrm{F}$ & Faraday \\
\hline FAS & Fractional area shortening \\
\hline GAPDH & Glyceraldehyde-3-phosphate dehydrogenase \\
\hline $\mathrm{H} / \mathrm{E}$ & Hematoxylin and eosin staining \\
\hline $\mathrm{HCl}$ & Hydrochloric acid \\
\hline HDAC & Histone deacetylases \\
\hline $\mathrm{HF}$ & Heart failure \\
\hline HR & Heart rate \\
\hline HRP & Horseradish peroxidase \\
\hline HW & Heart weight \\
\hline $\mathrm{Hz}$ & Hertz \\
\hline $\mathrm{I}_{\mathrm{Ca}}$ & L-type calcium current \\
\hline ICAM & Intercellular adhesion molecule \\
\hline ICD & Intercalated discs \\
\hline IF & Immunofluorescence \\
\hline $\mathrm{I}_{\mathrm{Na}}$ & Sodium current \\
\hline IV & Current-voltage \\
\hline $\mathrm{K}^{+}$ & Potassium \\
\hline $\mathrm{kDa}$ or $\mathrm{kD}$ & Kilodalton \\
\hline $\mathrm{KI}$ & Redox dead Cys17Ser knock-in mouse \\
\hline LTCC & L-type calcium channel \\
\hline LVH & Left ventricular hypertrophy \\
\hline LVIDd & Left ventricular inner diameter diastole \\
\hline LVIDs & Left ventricular inner diameter systole \\
\hline MAO & Monoamine oxidase \\
\hline MI & Myocardial infarction \\
\hline MTC & Masson's trichrome staining \\
\hline $\mathrm{Na}^{+}$ & Sodium \\
\hline NADP & Nicotinamide adenine dinucleotide phosphate \\
\hline NCX & Sodium-calcium exchanger \\
\hline NOS & Nitric oxide synthase \\
\hline NOX & NADPH oxidase \\
\hline OMP & Osmotic minipump \\
\hline OP & Operation \\
\hline OSA & Obstructive sleep apnea \\
\hline PBS & Phosphate-buffered saline \\
\hline PBST & Phosphate-buffered saline Tween 20 \\
\hline PCR & Polymerase chain reaction \\
\hline PKA & cAMP-dependent Protein kinase A \\
\hline PKA RI & cAMP-dependent Protein kinase A regulatory subunit I $\alpha$ \\
\hline PKA C & cAMP-dependent Protein kinase A catalytic subunit \\
\hline PKC & Protein kinase $\mathrm{C}$ \\
\hline PLB & Phospholamban \\
\hline
\end{tabular}




$\begin{array}{ll}\text { Prx } & \text { Peroxiredoxin } \\ \text { PVDF } & \text { Polyvinylidene difluoride } \\ \text { rcf } & \text { Relative centrifugal force } \\ \text { ROS } & \text { Reactive oxygen species } \\ \text { rpm } & \text { Revolutions per minute } \\ \text { RT } & \text { Room temperature } \\ \text { RV } & \text { Right ventricle } \\ \text { RyR2 } & \text { Ryanodine receptor 2 } \\ \text { SDS-PAGE } & \text { Sodium dodecylsulfate polyacrylamide gel electrophoresis } \\ \text { SEM } & \text { Standard error of mean } \\ \text { Ser } & \text { Serine } \\ \text { SERCA } & \text { Sarcoplasmic/endoplasmic reticulum Ca }{ }^{2+} \text { ATPase 2a } \\ \text { SOD } & \text { Superoxide dismutase } \\ \text { SR } & \text { Sarcoplasmic reticulum } \\ \text { TAC } & \text { Transverse aortic constriction } \\ \text { TBST } & \text { Tris-buffered saline Tween 20 } \\ \text { TEMED } & \text { Tetramethylethylenediamine } \\ \text { Thr } & \text { Threonine } \\ \text { TL } & \text { Tibia length } \\ \text { Tris } & \text { Tris (hydroxymethyl) aminomethane } \\ \text { Trx } & \text { Thioredoxin } \\ \text { TT } & \text { Transverse tubules } \\ \text { V } & \text { Volt } \\ \text { VCAM } & \text { Vascular cell adhesion molecule } \\ \text { VEGF } & \text { Vascular endothelial growth factor } \\ \text { VF } & \text { Ventricular fibrillation } \\ \text { VPR } & \text { Volume-pressure recording } \\ \text { VT } & \text { Ventricular tachycardia } \\ \text { WB } & \text { Western blot } \\ \text { WT } & \text { Wild type mouse } \\ \text { XO } & \text { Xanthine oxidase } \\ \alpha-\text { MHC } & \text { Myosin heavy chain alpha } \\ \beta_{1}-\text { AR } & \beta_{1} \text {-adrenergic receptor } \\ \beta_{2}-\text { AR } & \end{array}$


Abstract 
L-type $\mathrm{Ca}^{2+}$ channels (LTCC) are known to be involved in the dysregulation of intracellular $\mathrm{Ca}^{2+}$ handling in heart failure (HF) contributing to contractile dysfunction and arrhythmias. Beside alteration in subcellular localization, reduced LTCC function may be a consequence of altered posttranslational modification. It is known that cAMP-dependent protein kinase A (PKA) can phosphorylate LTCC resulting in increased $\mathrm{Ca}^{2+}$ current $\left(\mathrm{I}_{\mathrm{Ca}}\right)$. PKA-dependent LTCC phosphorylation has been shown to occur upon acute pressure overload partly compensating for reduced $\mathrm{Ca}^{2+}$ release from sarcoplasmic reticulum (SR). However, the mechanisms of PKA-dependent LTCC activation upon pressure overload are inadequately understood. Recent data suggest that PKA can be activated upon direct oxidation of its regulatory subunit (RI) without binding to its physiologic agonist, cAMP. In this thesis, I have investigated the pathophysiological relevance of this oxidative PKA activation for intracellular $\mathrm{Ca}^{2+}$ homeostasis, contractile function, and arrhythmogenesis upon pressure overload. Here, a novel PKA redox-dead knock-in mouse line was used harboring a RI point mutation that results in the exchange of cysteine 17 with serine (KI). Oxidation of cysteine 17 with consequent intersubunit disulfide bridge, RI-RI dimer, formation is inhibited. The later is a prerequisite for catalytic subunit release and phosphorylation of target proteins, i.e. oxidative activation of the kinase. At baseline, no alterations in $\mathrm{Ca}^{2+}$ handling (FURA-2-loaded isolated cardiomyocytes) or contractile function (echocardiography) are observed in these mice. However, NAPDH oxidase 2 (NOX2)-dependent oxidants stimulated by angiotensin II (AngII, $1 \mu \mathrm{mol} / \mathrm{L}$ ) could not result in RI dimer (Western blotting) formation in KI. In accordance, ICa and $\mathrm{Ca}^{2+}$ transient amplitude were significantly smaller in KI mice in the presence of AngII. Similarly, pressure overload by transverse aortic constriction did not result in RI dimer formation in KI. Moreover, compared to WT mice KI mice displayed significantly reduced $\mathrm{I}_{\mathrm{Ca}}$ and $\mathrm{Ca}^{2+}$ transient amplitude with a significantly impaired ejection fraction (echocardiography) upon pressure overload. Western blot analyses revealed that AngII and pressure overload-dependent LTCC phosphorylation at the PKA site were inhibited. Beside disturbed contractile function, KI mice displayed severe QTc prolongation and increased the propensity for ventricular arrhythmias (ECG and programmed ventricular stimulation), which results in significantly reduced survival rates upon pressure overload. In summary, oxidative activation of PKA appears to be important for adaptation of the heart during increased afterload. Thus, stimulation of oxidative PKA activation could be a potential therapeutic option for patients with cardiac diseases, like heart failure. 


\section{List of Contents}

1.0. Chapter1: Introduction 01

1.1. Overview 02

1.2. Cardiac excitation-contraction (EC) coupling 03

1.3. Cardiac hypertrophy 05

1.4. Contractile dysfunction and arrhythmias 06

1.5. Heart failure 08

1.6. Calcium channels 10

1.6.1. Cardiac voltage-gated calcium channel $\quad 10$

1.6.2. Sarcoplasmic reticulum calcium release channel, RyR2 recep- 16 tor

1.7. Regulation of EC coupling by serine/threonine kinases 17

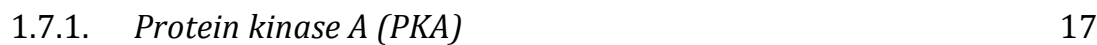

1.7.2 $\mathrm{Ca}^{2+} /$ Calmodulin kinase II (CaMKII) 24

1.7.3. Protein kinase C (PKC) 26

1.8. Oxidants 26

1.8.1. Sources of oxidants 28

1.8.2. How does the cell control oxidant detoxification? 32

1.8.3. Oxidative modifications of functionally important myocardial proteins and contractile dysfunction $\quad 34$

1.8.4. Redox-dependent gene transcription $\quad 35$

1.8.5. Oxidants can increase the propensity for cardiac arrhythmias 35

1.8.6. Oxidants promote hypertrophy 36

1.8.7. Oxidants induce apoptosis of cardiomyocytes 37

1.8.8. ROS production during ischemia-reperfusion and heart failure

1.9. Angiotensin II-mediated signaling in cardiomyocytes 39

1.10 Aim of the project $\quad 42$

2.0. Chapter2: Methods and Materials $\quad 43$

2.1. Experimental outline of the project 44

2.2. Generation of a novel 'redox dead' C17S RI knock-in (KI) mouse 44

2.3. gp91phox knockout (KO) mouse line 45

2.4. Genotyping of mice 45

2.5. Isolation of mouse ventricular cardiomyocytes 47

2.6. Whole-heart perfusion experiments 48

2.7. Mitochondrial and cytosolic ROS detection 48

2.8 Measurement of subcellular distribution of PKA RI using 50

2.9. Measurement of intracellular calcium in isolated ventricular myo$\begin{array}{ll}\text { cytes } & 51\end{array}$

2.10. Measurement of calcium sparks using confocal microscope 53

2.11. Evaluation of calcium channel function using patch-clamp experiments

2.1.2. Protein analysis of cardiomyocyte lysates and heart homogenates using western blot

2.13. In vitro cyclic adenosine monophosphate assay 59

2.14. In vitro histology experiments $\quad 60$ 
2.15. In vivo transverse aortic constriction of mice 61

2.16. In vivo osmotic minipump implantation 62

2.17. Blood pressure measurement 63

2.18. In vivo echocardiography 64

2.19. In vivo electrophysiological studies (EP studies) 64

2.20. Data analysis 66

$\begin{array}{ll}\text { Chemicals used in this study } & 67\end{array}$

$\begin{array}{ll}\text { Instruments used in this study } & 68\end{array}$

3.0. Chapter3: Results $\quad 69$

3.1. AngII induces cytosolic oxidant production 70

3.2. AngII induces mitochondrial oxidant production via NOX2 enzyme 71

3.3. Oxidative activation of type I PKA 72

3.4. Oxidation of PKA results in PKA-dependent LTCC phosphorylation 73

3.5. AngII-mediated cellular translocation of PKA RI subunits 74

3.6. Oxidized PKA RI is involved in AngII-dependent regulation of calcium signaling

3.7. Oxidized PKA RI is involved in the regulation of calcium channel gating

$\begin{array}{lll}\text { 3.8. WT and KI mice have showed similar cardiac function in vivo } & 80 \\ \text { 3.9. AngII induces cytosolic oxidant production in vivo } & 81\end{array}$

3.10. AngII-induced LTCC phosphorylation via PKA is absent in KI mice 82

3.11. AngII induces contractile dysfunction in mice lacking oxidative activation of PKA

3.12. AngII induces calcium channel dysregulation in mice lacking oxidative activation of PKA

3.13. Chronic AngII exposure does not increase the propensity for ventricular arrhythmias in mice

3.14. Oxidized PKA and heart failure

3.16. Type I PKA increases substrate phosphorylation upon pressure overload

3.17. TAC surgery severely impair contractile function in mice lacking oxidative activation of PKA

3.18. Calcium channel function is severely impaired in KI mice after TAC surgery

3.19. Pressure overload reduces the force of contraction of cardiomyocytes

3.20. KI mice have more arrhythmic propensity upon TAC surgery compared to WT

3.21. Human diseased patients show oxidative activation of PKA 


\section{List of Figures}

1.1. Schematic diagram of cardiac excitation-contraction coupling. 04

1.2. Contractile dysfunction and arrhythmogenesis in HF. 09

1.3. Transmembrane folding model structure of L-type $\mathrm{Ca}^{2+}$ channel. 12

$\begin{array}{ll}\text { 1.4. Cardiac } \mathrm{Ca}^{2+} \text { channel activation and availability. } & 14\end{array}$

1.5. $\quad \beta$-adrenergic modulation of cardiac L-type calcium channels via G-protein coupled receptors. 15

1.6. Model of the PKA RI $\alpha$ tetrameric holoenzyme. 19

1.7. Schematic diagram showing physiologic roles of PKA in EC coupling. 21

1.8. Schematic diagram showing activation pathways of type I protein kinase A. 24

1.9. Sources of ROS generation. 29

1.10. Schematic figure representing the major antioxidant system inside cells. 33

1.11. Schematic diagram showing role of ROS in EC coupling. 34

1.12. Reactive oxygen species (ROS) induces membrane excitability and ar-
rhythmia in cardiomyocytes via modulating calcium current.

1.13. Schematic diagram showing predicted role of AngII-mediated ROS in EC
coupling.

2.1. Schematic diagram showing experimental plans for the project. 44

2.2. DNA gel electrophoresis revealed different mice genotypes of PKA RI mouse line.

2.3. Oxidation of MitoSOX Red mitochondrial superoxide indicator to 2-hydroxy5-(triphenylphosphonium)hexylethidium by superoxide $\left(\mathrm{O}_{2}{ }^{*}\right)$.

2.4. Schematic diagram showing the procedure and protocol for whole cell $\mathrm{I}_{\mathrm{Ca}}$ measurement.

2.5. Schematic diagram showing the principle of competitive cAMP immunoassay.

2.6. The standard curve of supplied cAMP molecules obtained from immunoassay.

$\begin{array}{lll}\text { 3.1. } & \text { In vitro application of AngII increases cytosolic ROS production. } & 70 \\ \text { 3.2. } & \text { AngII-mediated mitochondrial ROS production. } & 71\end{array}$

$\begin{array}{ll}\text { 3.3. Oxidant-induced PKA RI dimer formation. } & 72\end{array}$

3.4. AngII-dependent LTCC phosphorylation is mediated by oxidative PKA activation.

3.5. Oxidant-induced translocation of PKA RI subunit.

3.6. AngII maintains $\mathrm{Ca}^{2+}$ transients via oxidative PKA activation. 75

3.7. Reduction of SR $\mathrm{Ca}^{2+}$ contents upon AngII. $\quad 76$

3.8. Spontaneous $\mathrm{Ca}^{2+}$ release does not depend on PKA oxidation. 77

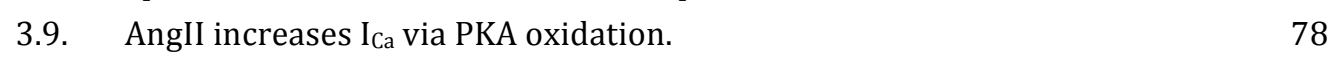

3.10. AngII increases open probability of calcium channel via oxidized PKA. $\quad 79$

3.11. Redox-dead PKA mice have a similar cardiac contractile function like WT at baseline.

3.12. Chronic AngII infusion induces oxidant production both in WT and KI. 82

3.13. Chronic infusion of AngII induces PKA RI dimer formation. 83

3.14. Chronic AngII-induced activation of PKA type I cause subsequent substrate phosphorylation.

3.15. Effects of AngII on CaMKII and PKC expression. $\quad 85$

3.16. Chronic AngII infusion alters cardiac function. 86

3.17. Oxidative PKA activation maintains $\mathrm{Ca}^{2+}$ channel function under chronic 
AngII infusion.

3.18. Oxidative PKA activation modulates $\mathrm{Ca}^{2+}$ transient but not $\mathrm{Ca}^{2+}$ spark upon chronic AngII infusion.

3.19. Chronic infusion of AngII to WT and KI mice do not induce arrhythmia. 91

3.20. Pressure overload induces ROS production in cardiomyocytes. 93

3.21. Pressure overload increases PKA RI dimer only in WT but not in KI mice. 94

3.22. Pressure overload-mediated PKA oxidation induces phosphorylation of downstream targets.

3.23. CaMKII and PKC expressions are not different in mice upon pressure overload.

3.24. In vivo echocardiography reveal impaired cardiac contraction upon pressure overload.

3.25. Pressure overload induces morphologic changes in the heart.

3.26. Redox-dead PKA mice have compromised calcium channel function after TAC surgery.

3.27. Pressure overload impairs calcium handling of isolated cardiomyocytes. 102

3.28 Pressure overload increases the arrhythmogenic vulnerability in KI mice. 103

3.29 Oxidation of PKA regulatory subunit at different human disease stages. 


\section{List of Tables}

2.1. Summary of PCR protocol for PKA RI mouse line. 46

2.2. Summary of PCR protocol for gp91phox mouse line. 46

2.3. Buffers and solutions used for mouse cardiomyocyte isolation. 47

2.4. The composition of solution used for fluorescence measurements. 50

2.5. The composition of solution used for epi-fluorescence measurements. 52

2.6. The composition of solutions used for patch-clamp experiments 55

2.7. The compositions of western blot lysis and sample buffers. 57

2.8. SDS-PAGE resolving and stacking gel compositions. 58

2.9. The composition of different buffers used in western blot. 58

2.10. List of antibodies used in this study. 59

2.11. The composition of solutions used in histochemistry experiments. 61

2.12. Representative echocardiographic parameters and their calculation formulas.

3.1. Summary of echocardiographic parameters of WT and KI mice at baseline condition (mean \pm SEM).

3.2. Summary of electrocardiographic parameters of WT and KI mice at baseline condition (mean \pm SEM).

3.3. Summary of echocardiographic parameters of WT and KI mice after two weeks of saline or AngII infusion (mean \pm SEM).

3.4. Summary of electrocardiographic parameters of WT and KI mice after two weeks of saline or AngII infusion (mean \pm SEM).

3.5. Summary of echocardiographic parameters of WT and KI mice after six weeks of sham and TAC surgery (mean \pm SEM).

3.6. Summary of electrocardiographic parameters of WT and KI mice after six weeks of sham and TAC surgery (mean \pm SEM). 


\section{Chapter 1 Introaluction}




\subsection{Overview}

The heart is central organ of the circulatory system, which pumps blood to different parts of the body using an intricate network of blood vessels, composed of arteries, veins, and capillaries. This pumping function is coordinated at the cellular level mostly by Calcium $\left(\mathrm{Ca}^{2+}\right)$, a critical physiological second messenger that mediates electrical activation, ion channel gating, excitation-contraction (EC), gene expression, hypertrophy and apoptosis of cardiac muscle. Intracellular $\mathrm{Ca}^{2+}$ concentrations are carefully regulated being at $\sim 100 \mathrm{nM}$ under resting conditions and increasing to $\sim 1 \mu \mathrm{M}$ during cardiac contraction, systole. However, localized $\mathrm{Ca}^{2+}$ concentrations are even higher inside the cardiomyocytes and can be reached up to $\sim 100 \mu \mathrm{M}$ during systole (Maier and Bers, 2002), (Bers and Guo, 2005). It is known that many factors can influence this highly regulated $\mathrm{Ca}^{2+}$ homeostasis. Strong evidence suggests that among these factors, exogenous and endogenous oxidants are crucially involved (Wagner et al., 2013).

Upon dysregulation, heart and blood vessels can initiate a variety of disease phenotypes, collectively known as cardiovascular diseases (CVDs). CVDs are the number one cause of death globally: more people die annually from CVDs than from any other reasons (World health organization, WHO). Among the CVDs, heart attack and stroke are usually acute events, which are mainly caused by a blockage that prevents blood from flowing to the heart or brain. However, the ultimate consequence of these diseases is heart failure, which means that the heart is unable to provide sufficient cardiac output to supply the metabolic demands of the organism. An estimated 17.5 million people died from CVDs in 2012, representing $31 \%$ of all global deaths. Of these deaths, an estimated 7.4 million were due to coronary heart disease and 6.7 million were due to stroke (WHO). Until now, two fatal pathways were reported to be responsible for this high mortality: 1) progressive decline in cardiac contractile function (pump failure) and 2) sudden cardiac death due to arrhythmias. The contraction abnormalities start at the cardiomyocyte and trabecular level, whereas arrhythmia can happen due to both $\mathrm{Ca}^{2+}$ handling and cellular ionic currents (Bers, 2001). It is projected that by 2030 , almost 23.6 million people will die from CVDs, mainly from heart disease and stroke (WHO). Thus, there is an ultimate need to identify 
the molecular mechanisms underlying to CVDs and HF. Therefore, this section will focus on the cellular basis of contractile dysfunction and arrhythmogenesis with particular emphasis on redox signaling in these settings.

\subsection{Cardiac excitation-contraction (EC) coupling}

$\mathrm{Ca}^{2+}$ is central to the regulation of cardiac contractile function. It links membrane excitation to cellular contraction in a process called excitation-contraction (EC) coupling (Reichel and Bleichert, 1959). In brief, EC coupling is initiated by depolarization of the sarcolemma of cardiomyocytes, which occurs during the upstroke of the cardiac action potential. The latter is mediated by rapid opening of cardiac voltage-gated $\mathrm{Na}^{+}$channels $\left(\mathrm{Na}_{\mathrm{v}} 1.5\right)$. These channels, however, undergo a short time-dependent (few milliseconds) conformational change that leads to the closure of the channel. In conjunction with the opening of transient outward potassium channels, the rapid closure of $\mathrm{Na}_{\mathrm{v}} 1.5$ results in a short repolarization (i.e. notch of the action potential), which is immediately followed by the opening of voltage-gated $\mathrm{Ca}^{2+}$ channels (LTCC). These channels stay open for a longer period (hundreds of milliseconds) allowing a substantial amount of $\mathrm{Ca}^{2+}$ ions to enter the cell and shaping the plateau phase of the action potential. If in close proximity-few nanometers, which is the case for the dyads (see below)-this $\mathrm{Ca}^{2+}$ influx triggers the opening of $\mathrm{Ca}^{2+}$ release channels of the sarcoplasmic reticulum (SR), named cardiac ryanodine receptors (RyR2). The $\mathrm{Ca}^{2+}$-influx-induced SR $\mathrm{Ca}^{2+}$ release (CICR) rapidly increases the cytosolic $\mathrm{Ca}^{2+}$ concentration to shape the $\mathrm{Ca}^{2+}$ transient, which is an increase in cytosolic free $\mathrm{Ca}^{2+}$ from about $100 \mathrm{nM}$ to about $1 \mu \mathrm{M}$ (Bers, 2002). The $\mathrm{Ca}^{2+}$ transient then activates the myofilaments, resulting in cellular contraction by binding $\mathrm{Ca}^{2+}$ to troponin $\mathrm{C}$. This $\mathrm{Ca}^{2+}$ bound troponin $\mathrm{C}$ in turn form cross bridges with Troponin $\mathrm{T}$, tropomyosin, and actin to produce cardiac contractile force (fig.1.1) (Bers, 2002).

At the end of the plateau phase of the cardiac action potential delayed rectifying potassium channels open. The resulting outward current leads to the repolarization of the membrane potential. Simultaneous time-dependent closure of LTCC and opening of inward rectifying potassium channels also contribute to the repolarization. Upon closure of LTCC, CICR stops, and two major $\mathrm{Ca}^{2+}$ elimination 
pathways remove the systolic $\mathrm{Ca}^{2+}$. The significant fraction of $\mathrm{Ca}^{2+}$ is taken up by

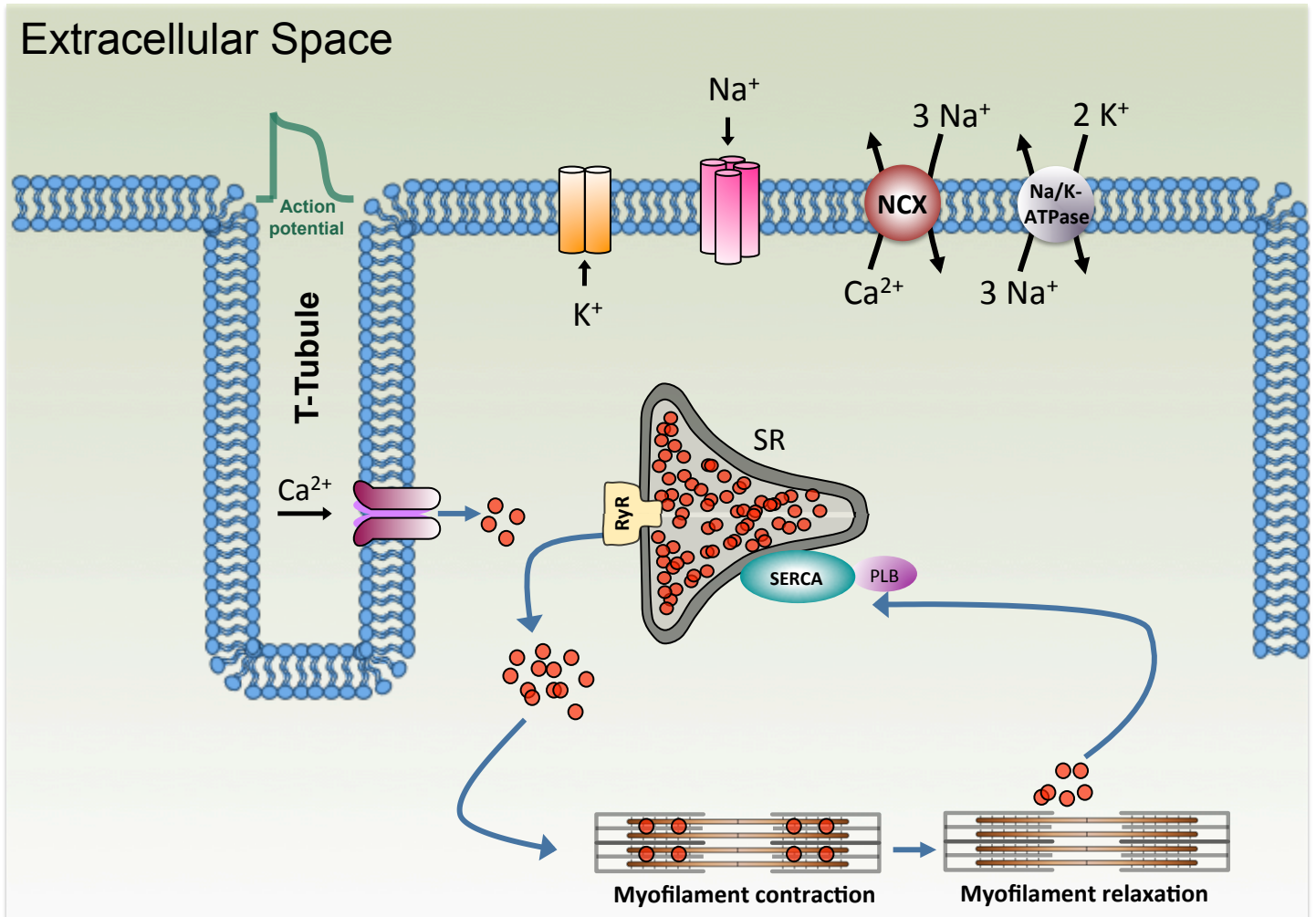

\section{Cytosol}

Figure 1.1. Schematic diagram of cardiac excitation-contraction coupling. $\mathrm{Ca}^{2+}$ ions enter through the L-type calcium channel into the cardiomyocytes and exits via sarcoplasmic reticulum $\mathrm{Ca}^{2+}$-ATPase (SERCA2a) and $\mathrm{Na}^{+} / \mathrm{Ca}^{2+}$-exchanger (NCX). Calcium entry mediates more $\mathrm{Ca}^{2+} \mathrm{re}^{-}$ lease from SR via RyR2 receptors. $\mathrm{Na}^{+}$ions enter through voltage-gated $\mathrm{Na}^{+}$channel and $\mathrm{NCX}$, while exits via $\mathrm{Na}^{+}-\mathrm{K}^{+}$ATPase in exchange for $\mathrm{K}^{+}$. Excess $\mathrm{K}^{+}$then exits the cell via $\mathrm{K}^{+}$channels. (modified from Wagner et al., 2013)

the SR $\mathrm{Ca}^{2+}$-ATPase (SERCA2a) into the SR, whereas smaller fraction exported into the extracellular space by the sarcolemmal $\mathrm{Na}^{+} / \mathrm{Ca}^{2+}$-exchanger (NCX) (Bers, 2002, (Bers, 2008; Fill and Copello, 2002). During steady-state, $\mathrm{Ca}^{2+}$ fluxes are balanced (Bers et al., 2003). Thus, the same amount of $\mathrm{Ca}^{2+}$ that entered the cell via LTCC is transferred out of the cell by NCX resulting in a stable diastolic SR $\mathrm{Ca}^{2+}$ content.

Organization of proteins to initiate CICR: Sarcoplasmic reticulums are sparse and flexibly organized with smaller saccular T-tubular (TT) enlargements at the cell surface and at junctions. The surface sarcolemma is physically continuous with the membrane of the T-tubule, and as such, the two combine to form the permeability barrier for $\mathrm{Ca}^{2+}$ between cytoplasm and the extracellular medium. At resting mouse heart, $\mathrm{Ca}^{2+}$ cycles between these compartments approximately 500 
times per minute. To maintain this $\mathrm{Ca}^{2+}$ cycle ion channels, pumps, and other proteins are specially organized on the membrane. For example, the density of LTCC is much higher in T-tubules compared to surface sarcolemma to continue this $\mathrm{Ca}^{2+}$ cycle. In cardiac muscle, these junctions are more apparent as dyads and can occur either at the surface sarcolemma or with the T-tubular membrane. RyR2 are organized in a distinct pattern on the SR, underneath the T-tubular membrane and organized along with LTCC. In mammalian ventricle, a typical cardiac couplon (ryanodine receptor clusters at TT and SR junctions) may have about 100 RyR2 and 10-25 LTCC (Bers, 2001).

This distinct organization of cellular organelles allows a rapid rise in cytoplasmic $\mathrm{Ca}^{2+}$ concentration, which is necessary for systolic contraction. For example, LTCC mediated 100 times increase of cleft $\mathrm{Ca}^{2+}$ from 0.1 to $\sim 10 \mu \mathrm{M}$, can be further increased to $\sim 100 \mu \mathrm{M}$ by RyR2 opening in the dyadic cleft, whereas global intracellular $\mathrm{Ca}^{2+}$ only reaches $\sim 1 \mu \mathrm{M}$ (at a later time). However, these local SR $\mathrm{Ca}^{2+}$ release events are synchronized, by action potentials and simultaneous activation of LTCC in all junctions, to produce a relatively homogenous increase in intracellular $\mathrm{Ca}^{2+}$ throughout the cytosol for sufficient force generation (Bers, 2008; Fill and Copello, 2002).

\subsection{Cardiac hypertrophy}

Cardiac hypertrophy is an adaptive process that helps the heart to generate sufficient force under increased workload and meet the needs of the body. In all cases, the increase in ventricular mass is paralleled by cardiomyocyte hypertrophy (Anversa and Kajstura, 1998; Soonpaa and Field, 1998). Ventricular hypertrophy can be either concentric or eccentric. Concentric hypertrophy, where the walls and cardiomyocytes thicken, but the chamber does not dilate, is especially common with pressure overload (i.e. when the heart cannot generate sufficient pressure to overcome elevated afterload). Eccentric hypertrophy, where the chamber becomes dilated and cardiomyocytes become elongated, is most common in volume overload (e.g. valvular insufficiencies) (Bers, 2001).

Ventricular hypertrophy occurs in response to numerous physiological and 
pathophysiological stimuli. In physiological settings, this can be considered as compensatory hypertrophy, where it increases cardiac function and stroke volume (and lower resting heart rate). In contrast, pathophysiological stimuli include hemodynamic load (pressure or volume) and neurohumoral stimuli (e.g. renin-angiotensin II, endothelin, adrenergic). At this point, the compensatory hypertrophic response may be inadequate, and further changes can become decompensatory, which contribute to the genesis of heart failure (HF) (Bers, 2001).

It has been reported that $\mathrm{Ca}^{2+}$ is involved in hypertrophic signaling via CaMKIICalcineurin-NFAT3 (Nuclear factor of activated T-cells) signaling pathway (Molkentin, 2000; Molkentin et al., 1998; Olson and Molkentin, 1999; Sussman et al., 1998). Under basal condition, calcineurin binds to the phosphorylated nuclear transcription factor NF-AT3. When calcineurin is activated by calcium bound calmodulin $\left(\mathrm{Ca}^{2+}-\mathrm{CaM}\right)$, it dephosphorylates NF-AT3, which is then translocated to the nucleus where it promotes gene expression. Transgenic over-expression of calcineurin or NF-AT3 in mice caused hypertrophy and HF, which can be blocked by inhibitors of calcineurin (cyclosporine and FK-506). Thus, cellular $\mathrm{Ca}^{2+} \mathrm{regu}-$ lation and its ability to regulate contractility play a central and interactive role in determining the ultimate cardiac phenotype. This is further revealed by the findings that enhancing $\mathrm{Ca}^{2+}$ transients and SR $\mathrm{Ca}^{2+}$-ATPase activity by SERCA2a gene transfer or Phospholamban gene ablation can inhibit hypertrophy and HF due to aortic banding (Minamisawa et al., 1999; Miyamoto et al., 2000).

\subsection{Contractile dysfunction and arrhythmias}

Cardiac electrical instability, e.g. due to hypertrophy, is responsible for increased arrhythmic vulnerability of heart tissue. This is one of the main causes of death after heart failure and myocardial infarction. Many of the cardiac events are proarrhythmogenic. For example, immediately after initial repolarization phase of the action potential, early afterdepolarizations (EAD) may occur due to increased late $\mathrm{Na}^{+}$current (late $\mathrm{I}_{\mathrm{Na}}$ ), This can consequentially prolong action potential (AP) duration (Sossalla et al., 2011; Wagner et al., 2015) (Wagner et al., 2011) (Hashambhoy et al., 2011). This long AP can inhibit $I_{C a}$ during cytosolic $\mathrm{Ca}^{2+}$ transient. However, subsequent reduction of $\mathrm{Ca}^{2+}$ can reactivate the $\mathrm{I}_{\mathrm{Ca}}$ 
again, which may also lead to EAD (January and Riddle, 1989). Moreover, elevated transient outward $\mathrm{K}^{+}$current can also augment EAD occurrences by reducing the voltage of the action potential plateau, which slows repolarizing currents and, at the same time, reactivates depolarizing $\mathrm{Ca}^{2+}$ currents via LTCC (Zhao et al., 2012). In addition, $\mathrm{Ca}^{2+}$ channel activation at more negative membrane potential (Em), inactivation at more positive Em and slower inactivation, all promotes the increase of $\mathrm{I}_{\mathrm{Ca}}$ window current. Because of this window current, $\mathrm{Ca}^{2+}$ channel conductance gets increased, which can cause depolarization, arrhythmia and heart failure as well (Pelzmann et al., 1998).

Beside early after depolarization, diastolic membrane instability can also lead to a triggered action potential. This late instability is called delayed after depolarization (DAD). The molecular reason for DAD is cytosolic and SR $\mathrm{Ca}^{2+}$ overloads that may cause spontaneous SR $\mathrm{Ca}^{2+}$ release. It has been reported that increasing SR $\mathrm{Ca}^{2+}$ raises the sensitivity of RyR2 to cytosolic $\mathrm{Ca}^{2+}$ (Tripathy and Meissner, 1996; Xu et al., 1998a; Xu et al., 1998b). Moreover, hyper-phosphorylated or oxidized RyR2 can also facilitate the $\mathrm{Ca}^{2+}$ release process (Belevych et al., 2012) (Sag et al., 2009). This elevated cytosolic $\mathrm{Ca}^{2+}$ can activate transient inward current mediated depolarization of the membrane and after contraction. Both of these, RyR2-mediated $\mathrm{Ca}^{2+}$ release and transient inward currents, are responsible for DAD. Ultimately, leading to a smaller $\mathrm{Ca}^{2+}$ transient amplitude and weaker cellular contraction and arrhythmia.

This RyR2-mediated disturbed $\mathrm{Ca}^{2+}$ handling is further augmented by NCX activity, which is increased due to diastolic $\mathrm{Ca}^{2+}$ overload, ultimately leads to a local depolarization and electrical instability. Like RyR2, modifications of NCX via either oxidation or kinase-mediated phosphorylation (which can also undergo oxidative activation) may play an important role in this regard (Foteinou et al., 2015; Nagy et al., 2014).

Moreover, activated CaMKII can also induce arrhythmia via modulating different ion channels. Interestingly, arrhythmia can be attenuated by the inhibition of late $\mathrm{I}_{\mathrm{Na}}$ (tetrodotoxin, ranolazine) or by CaMKII (Fukuda et al., 2005; Wagner et al., 
2006). Other reports suggested that $\mathrm{H}_{2} \mathrm{O}_{2}$-induced $\mathrm{Na}^{+}$overload develops more slowly and arrhythmia is less frequent in CaMKII-KO mice, while CaMKII $\delta_{C}$ overexpression had high $\mathrm{I}_{\mathrm{Na}}$ and showed more adverse effects (Wagner et al., 2011). These distinctly indicate that oxidative conditions can activate CaMKII, which alter $\mathrm{Na}_{\mathrm{v}} 1.5$ gating to mediate $\mathrm{Na}^{+}$overload, resulting in a pathologic outcome.

\subsection{Heart failure}

Heart failure (HF) is the condition when the heart stops to pump sufficiently enough to supply blood throughout the body. It has been found that cardiomyocytes have less positive (or even negative) force-frequency relationship in failing vs. non-failing hearts. Reduced twitch force and cardiomyocyte contractions are mainly responsible for this phenotype. These force changes are paralleled to changes in $\mathrm{Ca}^{2+}$ transient amplitudes and slowing of the rate of relaxation (Bers, 2001). Feldman et al. showed data suggesting that down-regulation of SERCA2a expression might mark the transition from hypertrophy to HF (Feldman et al., 1993). In HF, reduction in thyroid hormone results in decreased SERCA2a but increased PLB expression. Thus, significantly reduced SERCA2a: PLB ratio and profoundly depresses SR Ca ${ }^{2+}$ transport (Hamilton et al., 1990 \{Kiss, 1994 \#585; Ojamaa et al., 2000). It has been reported that myofilament $\mathrm{Ca}^{2+}$ sensitivity also decreased in $\mathrm{HF}$, which can reduce the force of contraction even further (Fan et al., 1997; Perez et al., 1999). In agreement with this Mukherjee et al., have been reported a decrease in peak $I_{C a}$ density during HF (Mukherjee and Spinale, 1998). This lower $\mathrm{I}_{\mathrm{Ca}}$ along with increased RyR2 spark frequency and NCX up-regulation (Studer et al., 1994) may also contribute to a lower SR Ca ${ }^{2+}$ content in HF. Marx et al. reported that this increase in spark frequency is due to hyperphosphorylation of RyR2 at PKA site as well as less phosphatase association with the RyR2 receptor (fig.1.2) (Marx et al., 2000).

Moreover, action potentials also get prolonged in HF (Bers, 2001). Smaller $\mathrm{Ca}^{2+}$ transients along with long AP in $\mathrm{HF}$, causes greater $\mathrm{Ca}^{2+}$ influx via $\mathrm{Na}^{+} / \mathrm{Ca}^{2+}$ exchanger (Dipla et al., 1999). However, this is only true at low heart rates when AP duration is unusually prolonged. This may also explain why the $\mathrm{Ca}^{2+}$ transients and contractile force are less depressed compared to control at low heart 
rates.

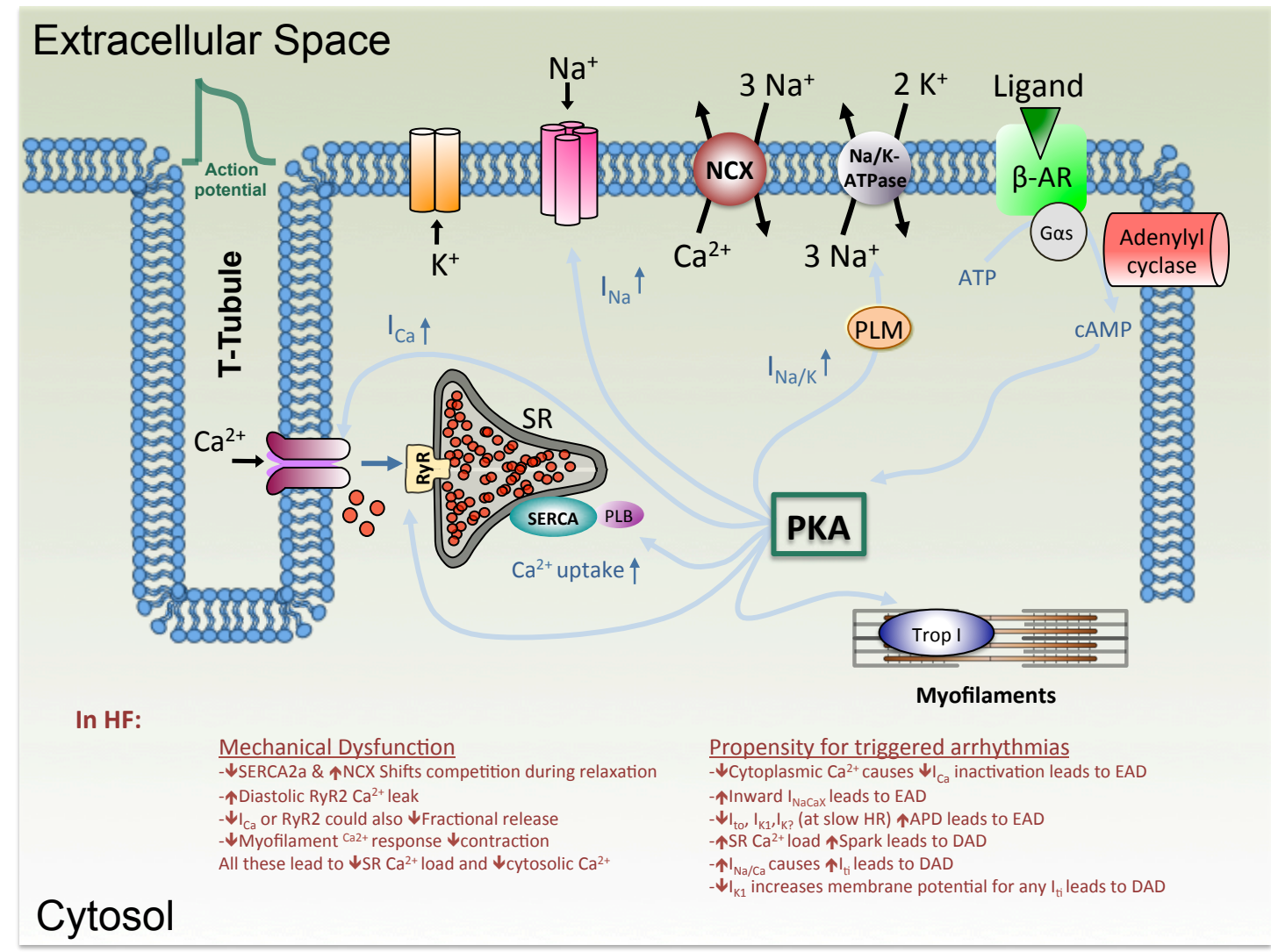

Figure 1.2. Contractile dysfunction and arrhythmogenesis in HF. The contractile dysfunction results from a reduction in SR Ca ${ }^{2+}$ content (due to reduced SR Ca ${ }^{2+}$-ATPase, increased $\mathrm{Na}^{+} / \mathrm{Ca}^{2+}$ exchange, and increased diastolic SR $\mathrm{Ca}^{2+}$ leak). Arrhythmogenesis may be triggered by either early or delayed afterdepolarizations (EADs or DADs). EADs are more likely at longer AP duration or due to mentioned factors. DADs are due to spontaneous $\mathrm{SR} \mathrm{Ca}^{2+}$ release. (modified from Wagner et al., 2013)

Thus, it seems likely that $\mathrm{Na}^{+} / \mathrm{Ca}^{2+}$ exchanger upregulation is an important factor in altered $\mathrm{Ca}^{2+}$ handling in HF. However, NCX function is critically dependent on the level of cytosolic $\mathrm{Na}^{+}$, which is, in turn, reliant on the activity of the $\mathrm{Na}^{+} / \mathrm{K}^{+}-$ ATPase. It has been reported that $\mathrm{Na}^{+} / \mathrm{K}^{+}$-ATPase expression is reduced in $\mathrm{HF}$ (Dixon et al., 1992; Schwinger et al., 1999; Semb et al., 1998), which could ultimately elevate cytosolic $\mathrm{Na}^{+}$(Pieske et al., 2002). This leads to less $\mathrm{Ca}^{2+}$ extrusion and greater $\mathrm{Ca}^{2+}$ influx via $\mathrm{Na}^{+} / \mathrm{Ca}^{2+}$ exchanger. Thus, elevated $\mathrm{Na}^{+}$can partly offset an even greater depression of $\mathrm{Ca}^{2+}$ transients and contractile function (Bers, 2001). In human HF, ventricular tachycardia (VT) is mostly initiated by nonreentrant mechanisms, like EADs or DADs, which is true for $100 \%$ of VT in nonischemic HF and 50\% in post-ischemic HF (Pogwizd et al., 1992; Pogwizd et al., 1998). In heart failure, low heart rates increased the probability that $I_{C a}$ has recovered from inactivation and prolonged the AP to induce EAD. However, at 
physiological frequencies, AP duration is not always so prolonged in HF. Additionally, DADs are also reported in HF. The key facets of HF that contribute to DAD-induced triggered arrhythmias are: 1) increased $\mathrm{Na}^{+} / \mathrm{Ca}^{2+}$ exchanger function, 2) reduced inward rectifier current, $\mathrm{I}_{\mathrm{K} 1}$ and 3 ) residual $\beta$-AR activity (to sufficiently increase $\mathrm{SR} \mathrm{Ca}^{2+}$ load for spontaneous $\mathrm{Ca}^{2+}$ release). Indeed, with the loss of $\beta$-AR responsiveness in very late-stage $\mathrm{HF}$, arrhythmias are less common (Bers, 2001).

\subsection{Calcium channels}

There are two types of calcium release channels, which play a prominent role in the excitation-contraction coupling: voltage-gated calcium channel and sarcoplasmic reticulum calcium release channel.

\subsubsection{Cardiac voltage-gated calcium channel}

Nowycky et al. characterized voltage-gated $\mathrm{Ca}^{2+}$ channels in dorsal root ganglion cells at 1985 and who divided them into three groups. L-type $\mathrm{Ca}^{2+}$ channels (LTCC), which have a large conductance ( $\sim 25 \mathrm{pS}$ in $\left.110 \mathrm{mM} \mathrm{Ba}^{2+}\right)$, long lasting openings (with $\mathrm{Ba}$ as the charge carrier), sensitivity to 1,4-dihydropyridines (DHPs) and activation at larger depolarizations (i.e. at more positive Em). T-type $\mathrm{Ca}^{2+}$ channels have, on the other hand, tiny conductance ( $\sim 8 \mathrm{pS}$ in $\left.110 \mathrm{mM} \mathrm{Ba}^{2+}\right)$, transient openings, insensitivity to DHPs, and activation at more negative Em. Ntype $\mathrm{Ca}^{2+}$ channels are neither $\mathrm{T}$ nor $\mathrm{L}$, are predominantly found in neurons and are intermediate in conductance and voltage dependence (Nowycky et al., 1985). However, these groupings are very superficial, and there are large differences among L-type channels. For example, $\omega$-conotoxin can strongly inhibit neuronal L-type $\mathrm{Ca}^{2+}$ channels, but not cardiac or skeletal muscle L-type channels. Besides, the activation and inactivation kinetics in skeletal muscle L-type channels are $\sim 10$-fold slower than in cardiac muscle. Moreover, there are also other $\mathrm{Ca}^{2+}$ current types distinguished by electrophysiological and pharmacological phenotype (e.g. P/Q \& R), which are more prominent in neurons and neuroendocrine cells (Bers, 2001). 
Cardiac muscle contains both $\mathrm{L}-$ and $\mathrm{T}$-type $\mathrm{Ca}^{2+}$ channels but not $\mathrm{N}$-type channels. $\mathrm{I}_{\mathrm{Ca}} \mathrm{L}$ is ubiquitous in cardiac myocytes, whereas cardiac $\mathrm{I}_{\mathrm{Ca}}, \mathrm{T}$ has localized distribution, like in atrial cells. It has been reported that T-type current is typically small or absent in ventricular cardiomyocytes but may be more prominent during development or in hypertrophy. This may reflect different functional roles, where $\mathrm{I}_{\mathrm{Ca}}, \mathrm{L}$ is more involved in triggering $\mathrm{SR} \mathrm{Ca}^{2+}$ release and refilling SR $\mathrm{Ca}^{2+}$ stores, rather than pacemaking activity (Bers, 2001). Throughout the thesis " $\mathrm{I}_{\mathrm{Ca}}$ " is used to indicate L-type $\mathrm{Ca}^{2+}$ current only, not the other $\mathrm{Ca}^{2+}$ currents.

Structure of calcium channel: The molecular composition of LTCC in cardiomyocytes includes the pore-forming $\alpha_{1 \mathrm{C}}$ (Cacna1c; referred to herein as $\alpha_{1 \mathrm{C}}$ ), beta $\beta$, aplpa2delta $\alpha_{2} \delta$, and gamma $\gamma$ subunits. The auxiliary subunits $\beta, \alpha_{2} \delta$, and $\gamma$ are involved in trafficking the pore-forming $\alpha_{1 \mathrm{C}}$ subunit to the sarcolemma and modulating the voltage dependence of channel gating (fig.1.3). $\alpha_{1}$ subunit consists of 4 homologous motifs (I-IV), each composed of 6 membrane-spanning $\alpha$-helices (termed S1 to S6) linked by variable cytoplasmic loops (linkers) between the S5 and S6 segments (fig.1.3). In total, ten $\alpha_{1}$ subunit genes have been identified and separated into four classes: $\mathrm{Ca}_{v} 1.1\left(\alpha_{1 \mathrm{~S}}\right), 1.2\left(\alpha_{1 \mathrm{C}}\right), 1.3\left(\alpha_{1 \mathrm{D}}\right)$, and $1.4\left(\alpha_{1 \mathrm{~F}}\right)$. Only the $\alpha_{1 \mathrm{C}}$ (dihydropyridine-sensitive [DHP-sensitive]) subunit is expressed at high levels in cardiac muscle. $\mathrm{Ca}_{\mathrm{v}} 2.1\left(\alpha_{1 \mathrm{~A}}\right), 2.2\left(\alpha_{1 \mathrm{~B}}\right)$, and $2.3\left(\alpha_{1 \mathrm{E}}\right)$ form P/Q-, N-, and possibly R-type channels, respectively, and are all found in brain (Bodi et al., 2005). In the heart, $\alpha_{1 C}, \alpha_{2} \delta$, and $\beta_{2}$ subunits have the molecular weight of 200 , 175 and $60 \mathrm{kDa}$ respectively (where the $\alpha_{2}-\delta$ split at about $150 \mathrm{kDa}$ as $\alpha_{2}$, and 30 $\mathrm{kDa}$ as $\delta$ subunit).

Activation and inactivation of calcium channel: Activation and inactivation of calcium channels prevent the breakdown of ionic gradients and determines action potential duration and the refractory period of excitable tissues (An and Zamponi, 2000-2013). $\mathrm{Ca}^{2+}$ channels can be activated and inactivated by changes in membrane potentials, Em (e.g. depolarization or hyperpolarization), binding of ligands (e.g. acetylcholine, ATP) or mechanical deformation (e.g. cell swelling). Cardiac $\mathrm{I}_{\mathrm{Ca}}$ is rapidly activated by depolarization, reaching a peak in $\sim 2-7 \mathrm{msec}$, depending on the temperature and Em. In contrast, inactivation is defined as a 
transition into a non-conducting state following channel opening, which depends on time-, Em- and cytoplasmic $\mathrm{Ca}^{2+}$ concentration (Bers, 2001). Inactivation rate is getting faster with stronger depolarization. Once inactivated, membrane repolarization is required for the channels to recover from inactivation. The rate of recovery from inactivation also depends on negative Em (faster at more negative Em).

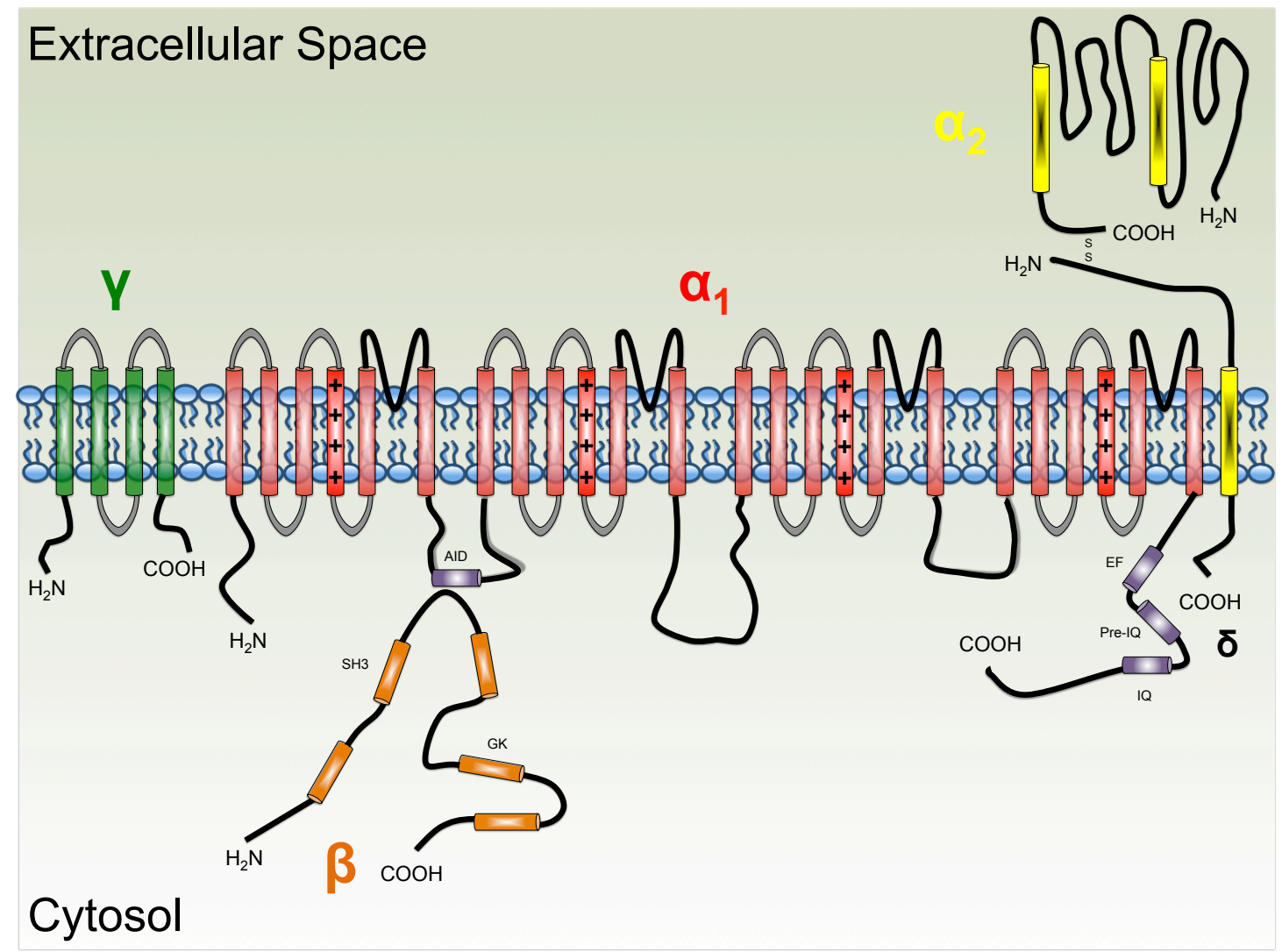

Figure 1.3. Transmembrane folding model structure of L-type $\mathrm{Ca}^{2+}$ channel. In the diagram, helices are depicted as cylinders; the lengths of lines correlate approximately to the lengths of the polypeptide segments. Pore-forming $\alpha 1_{\mathrm{C}}$ subunit illustrated as four homologous repeated domains (I-IV), each composed of six transmembrane segments. The cytoplasmic $\beta$ subunit is formed by two highly conserved domains, and the amino-terminal portion of the second conserved domain interacts with the I-II loop of $\alpha 1_{\mathrm{C}}$. The $\delta$ subunit has a single transmembrane segment with a short cytoplasmic $\mathrm{C}$-terminus and is linked by a disulfide bound to the extracellular, glycosylated $\alpha_{2}$ subunit. src homology 3 (SH3) motif, guanylate kinase-like (GK) motif, IQ motif, EF motif, $\alpha$ interaction domain (AID) are used to interact with other proteins and interacting partners of LTCC.

Based on work carried out in transient expression systems, the ability of voltagedependent calcium channels to inactivate appears to be an intrinsic feature of the $\alpha_{1}$ subunit since expression of this subunit alone produces inactivating currents (An and Zamponi, 2000-2013). Unlike in sodium and potassium channels, there have been no reports that showed a loss of inactivation following intracellular application of proteolytic enzymes such as pronase or trypsin, suggesting 
the possibility that inactivation of calcium channels could perhaps be fundamentally different from that of other voltage-gated channels and no involvement of cytoplasmic loop in channel inactivation (Bers, 2001). The apparent lack of involvement of a cytoplasmic loop suggested the possibility that calcium channel inactivation might occur via a pore collapse mediated by the S6 segment, similar to what has been proposed for slow inactivation of potassium channels (Durell et al., 1998; Liu et al., 1996; Ogielska et al., 1995). Taken together, the data of Berjukov et al. (Berjukow et al., 2001), Stotz et al. (Stotz et al., 2000), Zhang et al. (Zhang et al., 1994), Stotz and Zamponi (Stotz and Zamponi, 2001) suggested that all four S6 segments of the calcium channel $\alpha_{1}$ subunit contribute to inactivation. Furthermore, the domain I-II linker appears to be a key structure involved in this process (An and Zamponi, 2000-2013).

Moreover, calmodulin (CaM) that binds to the carboxy-terminal (1624-5 amino acids, a region between an EF-hand and IQ domain) of $\alpha_{1 C}$ subunit during diastole has been shown to involve in LTCC inactivation. During systole, this CaM will bind with high affinity to $\mathrm{Ca}^{2+}$, which have entered via LTCC or released by RyR2. Calcium bound CaM then interacts strongly with IQ domain of the $\mathrm{Ca}^{2+}$ channel to induce the $\mathrm{Ca}^{2+}$-dependent inactivation of $\mathrm{I}_{\mathrm{Ca}}$ (Bers and Guo, 2005). This act as an autoregulatory mechanism that limits $\mathrm{Ca}^{2+}$ entry under cytosolic $\mathrm{Ca}^{2+}$ overload (Choi, 1988; Orrenius et al., 1989; Orrenius and Nicotera, 1994).

Window $\mathrm{Ca}^{2+}$ current: During EC coupling, $\mathrm{Ca}^{2+}$ channels open and then gradually inactivated, resulting in a current that rises to a peak value from which it then decays. The overlapping of activation and inactivation of $\mathrm{Ca}^{2+}$ channel currents implied the existence of a steady state. This region of overlap is called as $\mathrm{Ca}^{2+}$ "window" current that exists near action potential plateau voltages (fig.1.4) (Brown et al., 1984; Cohen and Lederer, 1987; Josephson et al., 1984; Reuter and Scholz, 1977). Within this window, it is postulated that channel transitions may occur from inactivated to closed states (governed by the inactivation relation) and that channels may (re)open (governed by the activation relation) before inactivating again (Hirano et al., 1992). 
Interestingly, L-type $\mathrm{Ca}^{2+}$ window current has been proposed to be proarrhythmogenic. January and Riddle postulated that early afterdepolarizations result from the time- and voltage-dependent (re)opening of L-type $\mathrm{Ca}^{2+}$ channels within their window voltage range during the action potential plateau. This recovery of inward current shifts the balance of membrane currents toward depolarization (January and Riddle, 1989). When depolarization was initiated, additional L-type $\mathrm{Ca}^{2+}$ channels could then be recruited to open from a closed state(s), thereby augmenting the depolarizing $\mathrm{Ca}^{2+}$ current (Hirano et al., 1992) and mediates arrhythmia.

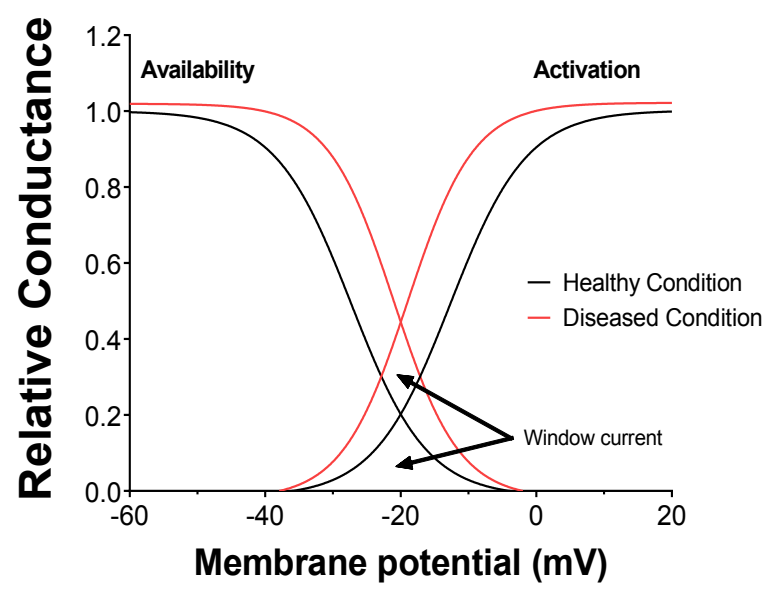

tential). (modified from Bers, 2001)
Figure 1.4. Cardiac $\mathrm{Ca}^{2+}$ channel activation and availability. $\mathrm{I}_{\mathrm{Ca}}$ availability is measured by depolarizing from $-90 \mathrm{mV}$ to the indicated Em for $1 \mathrm{sec}$ and then testing the remaining available $\mathrm{I}_{\mathrm{Ca}}$ at $\mathrm{Em}=0$ $\mathrm{mV}\left([\mathrm{Ca}]_{0}=1 \mathrm{mM}\right)$. The result is referred to as a steady state inactivation curve. Activation is measured by dividing the peak current by the apparent driving force (Em-Erev) according to Ohm's law $(\mathrm{G}=\mathrm{I} / \Delta \mathrm{V})$. Both curves are described by a Boltzmann relation. Black curves are for healthy and red curves indicating diseased condition (arbitrary data; Em: membrane potential, Erev: reversal po-

Regulation of calcium channel: Voltage-gated $\mathrm{Ca}^{2+}$ channels are responsible for translating action potential mediated electrical signal into the intracellular $\mathrm{Ca}^{2+}$ mediated signal (see above). Interestingly, LTCC can be regulated in its periphery because it forms large macromolecular complexes with several kinases, phosphatases, proteases and other proteins in the plasma membrane. It has been shown that CaMKII-mediated phosphorylation of $\beta_{2 \mathrm{~A}}$-subunit at T498 resulting in increased $\mathrm{I}_{\mathrm{Ca}}$ (Grueter et al., 2006). Moreover, PKA is known to phosphorylate S1928 in the $\alpha_{1 c}$-Subunit (fig.1.5) (Hudmon et al., 2005) (De Jongh et al., 1996; Yang et al., 2005). This phosphorylation causes a greater increase of $\mathrm{I}_{\mathrm{Ca}}$ at more negative potentials, which is due to negative shifts in the Em dependence of both activation and inactivation. This shift brings the Em-dependence of $\mathrm{I}_{\mathrm{Ca}}$ activation closer to the Em-dependence of $\mathrm{Ca}^{2+}$ channel gating current. 


\section{Extracellular Space}

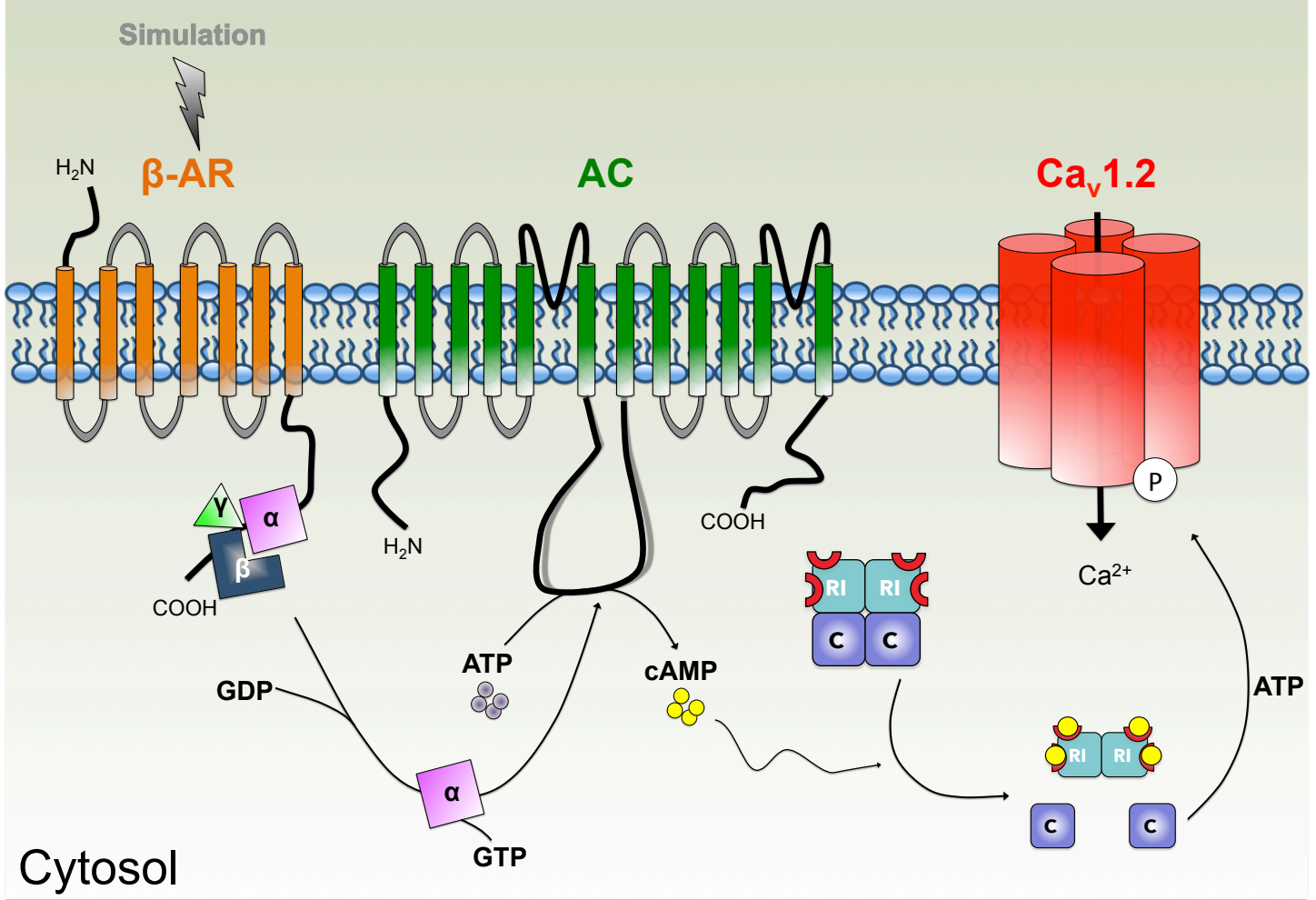

Figure 1.5. $\beta$-adrenergic modulation of cardiac L-type calcium channels via G-protein coupled receptors. Upon binding of agonist catecholamine to $\beta$-AR, stimulatory heterotrimeric $G$ protein get activated by inducing the exchange of guanosine diphosphate (GDP) for guanosine-5triphosphate (GTP) on $\mathrm{G} \alpha_{s}$. $\mathrm{G} \alpha_{s}$ dissociates from its $\mathrm{G}_{\beta \gamma}$ partner to stimulate the adenylyl cyclase (AC) present in the plasma membrane. In the AC catalyzes, the conversion of adenosine triphosphate (ATP) into cyclic 3,5-monophosphate (cAMP) that in turn activates the cAMP-dependent protein kinase (PKA) to phosphorylate $\alpha_{1 \mathrm{C}}\left(\mathrm{Ca}_{\mathrm{v}} 1.2\right)$ of LTCC.

In addition to Em shift, $\beta$-stimulation may increase $\mathrm{I}_{\mathrm{Ca}}$ by making the coupling between the charge movement and opening of the $\mathrm{Ca}^{2+}$ channels more efficient (Bers, 2001). However, in contrast to phosphorylation, the $\alpha_{1 \mathrm{c}}$-subunit has also been shown to be substrate for oxidation. Oxidation of -SH groups of $\alpha_{1 c^{-}}$ subunits lead to reduced peak I $\mathrm{C}_{\mathrm{Ca}}$ (Fearon et al., 1999; Gill et al., 1995; Goldhaber et al., 1989).

Interestingly, both CaMKII and PKA have shown to be activated by oxidants (see below). Thus, part of the oxidant-dependent regulation of $\mathrm{I}_{\mathrm{Ca}}$ may be mediated by oxidative activation of PKA or CaMKII. The relative contribution of each ICa regulatory pathway, however, is unknown, especially under pathophysiological conditions (Erickson et al., 2008) (Brennan et al., 2006) (Gopalakrishna and Anderson, 1989). The role of oxidant-activated PKA-dependent regulation of $I_{C a}$ 
is addressed in detail in this thesis (see below).

\subsubsection{Sarcoplasmic reticulum calcium release channel, RyR2 receptor}

The cardiac ryanodine receptor (RyR2) is a sarcoplasmic $\mathrm{Ca}^{2+}$ channel. It is a homotetrameric channel ( $\approx 2.2 \mathrm{MDa}$ ), a form of four RyR2 monomers (560 kDa) paired with four stabilizing proteins FKBP12.6. RyR2 is different from type 1 and 3 isoforms, which are predominantly expressed in other tissues. This channel is composed of 4 membrane-spanning subunits coupled to various regulatory proteins (Jorgensen et al., 1993). Calsequestrin, Triadin 1, and Junctin bind to RyR2 at the luminal SR membrane face, where they transmit information about SR $\mathrm{Ca}^{2+}$ content to RyR2 (Gyorke et al., 2004). In the cytoplasmic face, RyR2 is associated with PKA, CaMKII, protein phosphatases 1 and 2A, Calmodulin, and FKBP12.6 (Fill and Copello, 2002). RyR2 is closely localized with the LTCC in the t-tubule region, to maintain the $\mathrm{Ca}^{2+}$-induced $\mathrm{Ca}^{2+}$ release (CICR) of EC coupling. This CICR initiated when $\mathrm{Ca}^{2+}$ enter to the cell through LTCC, which in turn induces $\mathrm{Ca}^{2+}$ release from the SR via RyR2, leading to a substantial increase in cytosolic $\mathrm{Ca}^{2+}$ concentration. As a consequence systolic $\mathrm{Ca}^{2+}$ transient amplitude increases which ultimately activates the contractile system and generates the force for contraction.

Post-translational modifications of RyR2, via phosphorylation by CaMKII (at Ser2814/2815) and PKA (at Ser2808/2809), can mediate its activation. Recently it has been reported that kinases, which can modulate RyR2 function, can be oxidized and activated. Thus provide a unique mechanism where they indirectly enhance RyR2 function (Brennan et al., 2006; Eager and Dulhunty, 1998) (Maier and Bers, 2007).

In addition to the effector molecules (kinase and phosphatases), RyR2 function can also be modulated by redox-regulation. In the presence of oxidants, RyR2 monomer can form either disulfide bonds between any of its 21 free cysteines or other oxidized derivatives like S-nitrosylated products (Abramson and Salama, 1989) (Xu et al., 1998a) (Liu et al., 1994). It has been reported that at least 8 thiols per subunit need to be oxidized to mediate SR calcium release (Abramson and Salama, 1989; Boraso and Williams, 1994; Xu et al., 1998a). Moreover, S- 
nitrosylation of RyR2 at multiple cysteine residues can activate it, independently from phosphorylation (Burgoyne et al., 2012). The physiological consequences of these oxidative events are further explained by other groups where they have shown that oxidants (like 2,2'-dithiodipyridine, DTDP, $\mathrm{H}_{2} \mathrm{O}_{2}$ or glutathione deprivation) can increase RyR2 open probability $\left(\mathrm{P}_{\mathrm{o}}\right)$, which is responsible for increased $\mathrm{Ca}^{2+}$ loss from the SR. Consistently, reducing agents, such as Dithiothreitol (DTT), $\beta$-mercaptoethanol or antioxidants lead to decreased RyR2 activation (Anzai et al., 1998) (Terentyev et al., 2008) (Zable et al., 1997) (Kawakami and Okabe, 1998) (Mochizuki et al., 2007) (Yano et al., 2005).

\subsection{Regulation of EC coupling by serine/threonine kinases}

Ion channel gating controls EC coupling, which can be regulated by kinase and phosphatase-mediated posttranslational modifications.

\subsubsection{Protein kinase A (PKA)}

$\beta$-adrenergic signaling is critically involved in physiologic and pathophysiologic regulation of heart function to adapt to various kinds of stresses. Under threatening conditions (like a harmful event, attack, or threat to survival), the sympathetic nervous system causes secretion of catecholamine, especially norepinephrine and epinephrine for preparing the animal to react according to the situation. These physiologic comebacks are named as the fight-or-flight response. Among organs, the heart is mentionable because it has a remarkable capacity to react to altered physiologic demand by changing the rate at which it beats and the force with which it contracts, thereby changing its output. Contraction of heart is increased by sympathetic neurotransmitters-mediated activation of $\beta$-adrenergic receptors. Upon stimulation, these receptors increased cAMP production, which directly and indirectly (via activation of PKA and CaMKII) induces faster depolarization in sino-atrial node cells (which generate the action potentials that trigger cardiac contraction). Thus causes acceleration of heart rate (i.e., it has a "positive chronotropic effect") and stronger contraction (i.e., it has a "positive inotropic effect") and faster relaxation (i.e., it has a "positive lusitropic effect") in working cardiomyocytes (Eschenhagen, 2010). 
Initiation of $\beta$-adrenergic signaling: $\beta$-adrenergic receptors ( $\beta$-AR) are one kind of G-protein coupled receptors. The other form is $\alpha$ isoform. Receptor-associated G proteins consist of the $G_{\alpha}$ and the tightly associated $G_{\beta \gamma}$ subunits. There are many classes of $G_{\alpha}$ subunits: $G_{\alpha s}$ (G stimulatory), $G_{\alpha i}$ (G inhibitory), $G_{\alpha o}$ (G other), $\mathrm{G}_{\alpha \mathrm{q} / 11}, \mathrm{G}_{\alpha 12 / 13}$ etc. Both $\alpha$ and $\beta$-AR are subdivided into different subtypes namely $\alpha_{1}, \alpha_{2}, \beta_{1}, \beta_{2}, \beta_{3}$ and associated with various $G_{\alpha}$ proteins. $\alpha_{1}$ adrenergic receptor is coupled to $G_{\alpha q}$ involving in phospholipase $C$ signaling, $\alpha_{2}$ is coupled to $G_{\alpha i}$ proteins involving cAMP signaling, whereas, all the $\beta$ isoforms are linked to $G_{\alpha s}$ proteins also participating in cAMP signaling. Moreover, $\beta_{2}$ can also be coupled with $\mathrm{G}_{\alpha \mathrm{i}}$ proteins. The difference between $\mathrm{G}_{\alpha \mathrm{s}}$ and $\mathrm{G}_{\alpha \mathrm{i}}$ is that the former is involved in cAMP production whereas later one participates in cAMP degradation (Sprang et al., 2007).

Upon binding appropriate ligand to the receptor, the receptor has a conformational change and allows the $\mathrm{G}_{\alpha}$ subunit to be dissociated from $\mathrm{G}_{\beta \gamma}$ using the energy from GTP. Both $\mathrm{G}_{\alpha s}$-GTP and $\mathrm{G}_{\beta \gamma}$ can then activate different signaling cascades (or second messenger pathways) and effector proteins (Khafizov et al., 2009; Sprang et al., 2007). In the case of $\beta$-adrenergic receptor, after dissociation $\mathrm{G}_{\alpha \mathrm{s}}$-GTP moves to membrane bound adenylyl cyclase (AC). Using the energy of ATP, this activated AC then produces cAMP, which then acts as a second messenger inside the cell. The $\mathrm{G}_{\alpha}$ subunit will eventually hydrolyze the attached GTP to GDP by its intrinsic enzymatic activity, allowing it to re-associate with $\mathrm{G}_{\beta \gamma}$ and starting a new cycle.

Structure of PKA: cAMP-dependent protein kinase A (PKA) is one of the primary effectors of the $\beta$-receptors, which can be activated by cAMP. PKA is a homotetrameric protein composed of two regulatory and two catalytic subunits (fig. 1.6). To form a functional enzyme, homo-dimers of regulatory subunits bind to homo-dimers of catalytic subunits. There are two types of regulatory subunits, RI and RII. Two separate genes for each subunit encode $\alpha$ - and $\beta$-forms (Taylor et al., 1990), where $\alpha$ is predominant in the heart (Krall et al., 1999). In contrast four genes encoded for catalytic subunits, namely $C_{\alpha} / C_{\beta} / C_{\gamma}$ and PrKX (Beebe et al., 1990; Diskar et al., 2010), although $\alpha$ is the predominant gene expressed in 
the heart (Desseyn et al., 2000). However, each catalytic subunit consists of a small and a large lobe along with the active site, which forms a cleft between the two lobes. The small lobe provides the binding site for adenosine 5-triphosphate (ATP), and the large lobe provides a docking surface for peptide/protein substrates with serine/threonine residues, typically with an -R-R-X-S/T-X- motif (Kim et al., 2005). It has been reported that phosphorylation of catalytic subunit at T197 significantly increases the $\mathrm{k}_{\mathrm{m}}$ (Michaelis constant of the enzyme, which is inverse measure of affinity) of the enzyme toward substrates without significantly impacting on $\mathrm{k}_{\mathrm{c} a t}$ (measures the number of substrate molecules turned over per enzyme molecule per second) (Steichen et al., 2010).
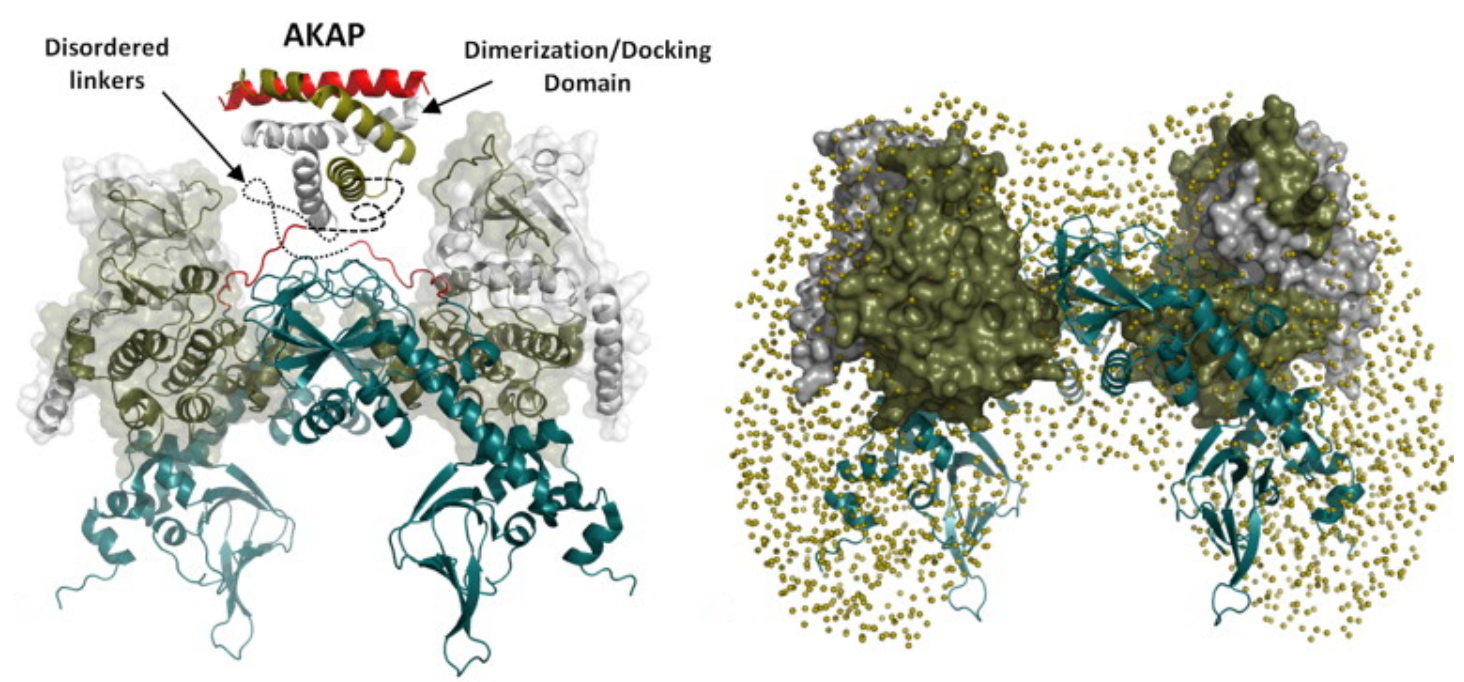

Figure 1.6. Model of PKA RI $\alpha$ tetrameric holoenzyme. Proposed positions of two PKA RI:C dimers correspond to the observed packing. Possible position of the dimerization/docking domain bound to the AKAP helix is illustrated using red color. Still unknown linkers between the DD domain and RI $\alpha$ CNB (cyclic nucleotide binding) domains are shown as dashed lines. $\mathrm{C}$ subunits are shown as tan colored surface, and R subunits are shown as the tan cartoon (right). (taken from Boettcher et al., 2011)

The regulatory subunits consist of a disordered linker region that connects the $\mathrm{N}$-terminal dimerization and docking domain (D/D) to the two 3', 5'- cyclic adenosine monophosphate (cAMP) binding domains (Taylor et al., 1990). The $\mathrm{D} / \mathrm{D}$ domain at the $\mathrm{N}$-terminal of the $\mathrm{R}$ subunits provides the interface for $\mathrm{R}$ subunit homo-dimerization, and is also the point of contact between PKA and Akinase anchoring proteins (AKAPs), scaffold proteins that tether PKA to specific subcellular locations to regulate localization, substrate specificity and catalytic activity of PKA (Newlon et al., 2001). 
Activation and downstream phosphorylation by PKA: According to the isoform of the regulatory subunits, two types of PKA have been described: PKA type I (for RI subunit) and type II (for RII subunit). To maintain the holoenzyme in the inactive state, RI and II tether to catalytic subunit through inhibitory domains presented either as a pseudo-substrate site in RI (Poteet-Smith et al., 1997) or the phosphorylatable Ser96 in RII (Carmichael et al., 1982). Upon $\beta$-adrenergic stimulation, adenylate cyclase augmented cAMP production. Increased binding of cAMP to RI or RII subunits (4 molecules of cAMP bind one subunit) result in the release of catalytic subunits and downstream target protein phosphorylation. It is known that PKA can phosphorylate and activated several $\mathrm{Ca}^{2+}$-cycle regulatory proteins, including LTCC (at S1928), RyR2 (at S2808), cardiac Na channel (at S526, S529), troponin I (at S22/23) and PLB (at S16) (fig.1.7) (Bers, 2001). Here in this thesis type I PKA means PKA holoenzyme containing $\mathrm{RI}_{\alpha}$ subunit, similarly, $\mathrm{RI}_{\alpha}$ subunit indicated as $\mathrm{RI}$.

Localization of PKA: Type I PKA is mainly localized in the cytosol, whereas type II appears to be primarily targeted to A-kinase anchoring proteins (AKAPs) that are localized to distinct subcellular compartments (Corbin et al., 1977; Scholten et al., 2007). There are significant uncertainties about target specificity of the PKA subtypes. For example, Burton et al. reported that PKA RII, which interacts with LTCC via AKAP 79, is not essential to modulate LTCC function. PKA RI, which has a 500-fold lower affinity for AKAP 79, can compensate the absence of PKA RII for LTCC modulation in skeletal muscle cells of RII ${ }_{\alpha} \mathrm{KO}$ mice (Burton et al., 1997). Jones et al. reported a similar type of results, where they had shown that cardiomyocyte-specific knockout of AKAP7, which anchored RII to LTCC, does not have any impact on $I_{\mathrm{Ca}}$ regulation (Jones et al., 2012). This implies that RI can counterbalance the role of RII at some extent.

Compensatory role of type I PKA: It has been reported previously that, RI especially the $\alpha$ isoform, has unique capacity to significantly compensate PKA activity in tissues where the other regulatory subunits are expressed, including brain, brown and white adipose tissue, skeletal muscle, and sperm, confirmed by gene knockouts of the three other regulatory subunits in mice (Amieux and McKnight, 
2002). Interestingly, in brown adipose tissue (BAT) increase in $\mathrm{RI}_{\alpha}$ subunit (typically scarce in BAT, RI beta is not expressed, and RII alpha is barely detected) as a compensation of RII beta KO, shows more avidity and affinity to cAMP compared to WT enzyme. KO is protected against many adverse effects of high-fat diet. Moreover, this isoform switched $\mathrm{RI}_{\alpha}$ elevates the metabolic rate and induces uncoupling protein in brown adipose tissue (Cummings et al., 1996).

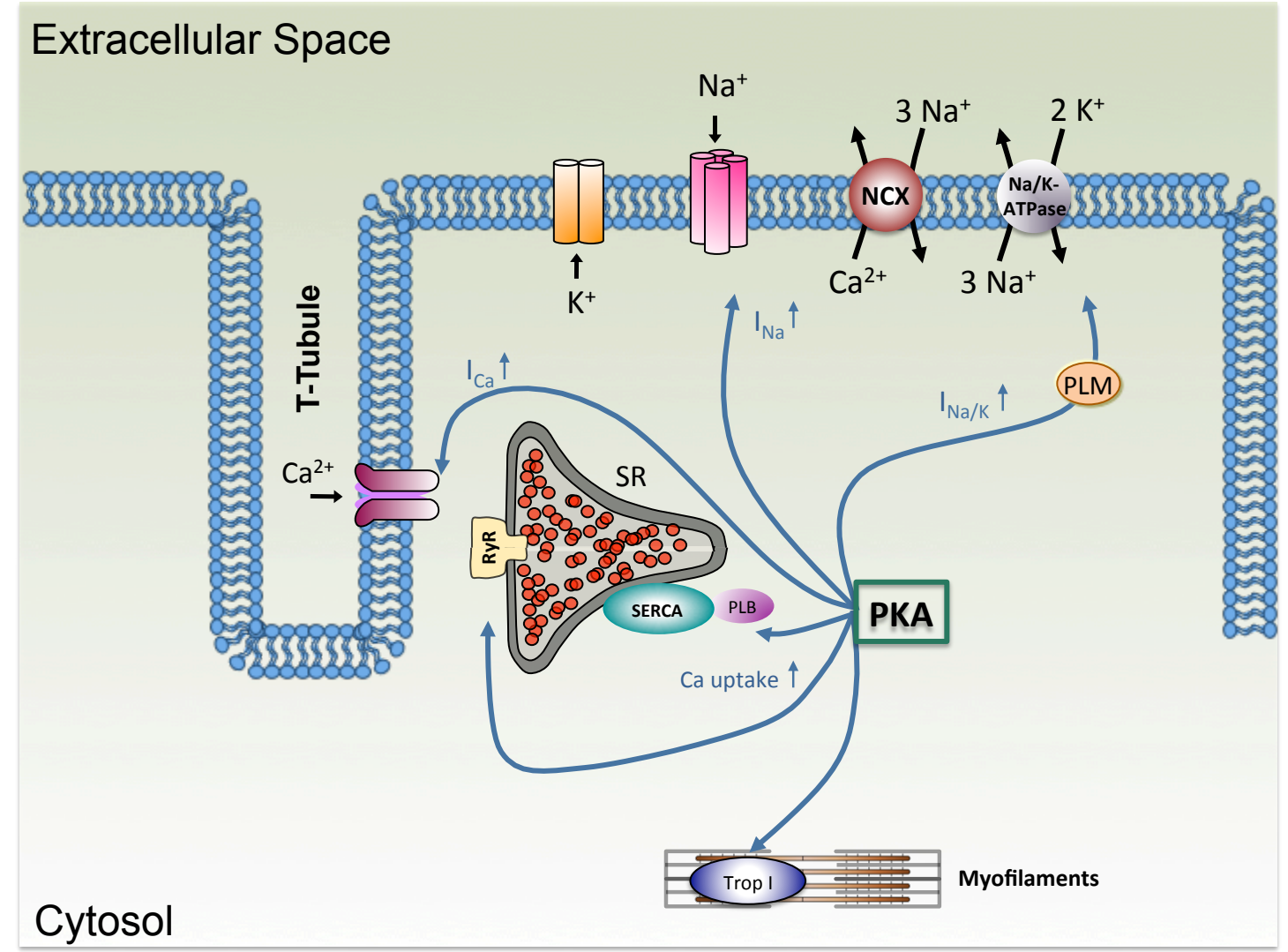

Figure 1.7. Schematic diagram showing physiologic roles of PKA in EC coupling. Upon activation, PKA can phosphorylate several targets of EC coupling for example LTCC, RyR2, cardiac Na channel, troponin I, PLB and modulates their functions. (modified from Wagner et al., 2013)

$\mathrm{RI}_{\alpha}$ subunits' compensatory role is further confirmed because it is only when catalytic subunit levels exceed RII subunit levels, that type I holoenzyme forms. Moreover, overexpression of RII completely eliminates RI holoenzyme (Clegg et al., 1989). Consistent with these data is that the rate of turnover of free $\mathrm{RI}_{\alpha}$ is also very high in cytosol at basal conditions (Orellana and McKnight, 1990). Its essential requirement was further proven by the knockout of $\mathrm{RI}_{\alpha}$ in mice, which results in early embryonic lethality due to aberrant cardiac morphogenesis (Amieux and McKnight, 2002) (which is may be due to uncontrolled catalytic subunit activation). 
Role of PKA in physiologic conditions: Phosphorylation of LTCC and RyR2 increases $\mathrm{I}_{\mathrm{Ca}}$ and CICR resulting in increased $\mathrm{Ca}^{2+}$ transient amplitude and contractile force (positive inotropic effect). It has been found that $\beta$-AR mediated PKA phosphorylation increases $\mathrm{I}_{\mathrm{Ca}}$ amplitude and shifts activation to more negative Em. Furthermore, current also inactivates quickly (owing to greater $\mathrm{Ca}^{2+}$ reflux and release). Thus there is only a little increase in $\mathrm{I}_{\mathrm{Ca}}$ during action potential (Bers, 2008). Moreover, it has been found that $I_{\mathrm{Ca}}$ density is unchanged in canine tachycardia-induced heart failure model. This unaltered $\mathrm{I}_{\mathrm{Ca}}$ is may be due to unchanged PKA function, as type I PKA can compensate type II PKA function (Burton et al., 1997). However, if only specific PKA type or target is altered (for example type I PKA), detrimental effects may happen (as mentioned is this thesis, where redox active PKA specifically alter LTCC).

Moreover, PKA-dependent PLB phosphorylation results in increased SERCA2a activity. The latter could increase $\mathrm{SR} \mathrm{Ca}^{2+}$ content and make more $\mathrm{Ca}^{2+}$ available for the consecutive CICR (positive inotropic effect). It also enhances the speed of $\mathrm{Ca}^{2+}$ removal thus stimulating relaxation (positive lusitropic effect). Moreover, PKA-dependent phosphorylation of troponin I reduces myofilament $\mathrm{Ca}^{2+}$ sensitivity, which further contributes to faster relaxation (positive lusitropic effect). (Ramirez-Correa et al., 2010) (Schulman and Greengard, 1978; Schworer et al., 1986). Another target of PKA is $\mathrm{Na}_{\mathrm{v}} 1.5$, which is phosphorylated at $\mathrm{S} 526$ and S529 (Murphy et al., 1996). In consequence of phosphorylation, $\mathrm{Na}_{v} 1.5$ trafficking to the sarcolemma is enhanced resulting in increased peak $\mathrm{I}_{\mathrm{Na}}$ amplitude. The larger peak $\mathrm{I}_{\mathrm{Na}}$ improves ventricular conduction velocity by faster AP upstroke (Scheuer, 2011). These all help to maintain the force-frequency-relationship of cardiomyocytes (Shattock and Matsuura, 1993). We have shown that beside beta-adrenergic stimulation, exposure of cardiomyocytes to AngII, which increases ROS production, results in increased $\mathrm{I}_{\mathrm{Ca}} \mathrm{I}_{\mathrm{Na}}$, and action potential upstroke velocity. These effects could be blocked by PKA inhibition (Wagner et al., 2014).

Role of PKA in pathophysiologic conditions: In contrast to the physiologic role of PKA, its significance on EC coupling during pathophysiological conditions is less clear. It has been shown that chronically elevated adrenergic agonist activity (as 
found in heart failure) leads to down-regulation of $\beta$-AR signaling due to negative feedback to this pathway (Bunemann et al., 1999; Lefkowitz, 1998; Summers et al., 1997) (Bohm and Lohse, 1994; Ungerer et al., 1993). Wang et al. believed that there is a time-dependent switch from the PKA-dominant pathway to the CaMKII-dominant pathway after receptor stimulation, where the sustained responses were largely PKA- independent, and were sensitive to CaMKII (Wang et al., 2004). Moreover, even though with this elevated adrenergic agonist level, failing hearts could not produce sufficient cAMP to maintain proper cardiac muscle contraction, indicating a miscommunication between the receptor and underlying coupling proteins (Lutz et al., 2001). As indicated before that $\beta_{1}$ receptors are responsible for cardiac cell surface and $\beta_{2}$ receptors are mediating T-tubular signaling (Zakhary et al., 2000). Interestingly, Nikolaev et al. showed a loss of ttubules with a massive redistribution of $\beta$-receptors in rat heart failure model. According to their report, during heart failure $\beta_{1}$ receptors keep staying on the cell surface, whereas $\beta_{2}$-AR rearranged from the T-tubules to the cell crest in failing cardiomyocytes and loss proper PKA localization (Nikolaev et al., 2010). Moreover, they have reported that in $\beta_{2}$-ARs in detubulated areas of failing cardiomyocytes produced diffuse cAMP signaling that propagated throughout the entire cytosol and losses its beneficial impacts on the cell (Nikolaev et al., 2010; Zakhary et al., 2000).

Therefore, it can be summarized that a paradoxical reduction of PKA-mediated signaling may happen during heart failure, despite the fact that $\beta$-AR agonists are present in large amounts in the blood and extracellular space. The inability of the heart to maintain physiologic PKA-dependent modulation of EC coupling may contribute to the disease progression.

Novel PKA activation pathway: Recently, an alternative PKA activation pathway has been reported, which is independent of beta-adrenergic stimulation and cAMP levels (Brennan et al., 2006). Oxidation of C17 and C38 of the RI homodimer could lead to intermolecular RI-RI disulfide bond formation, where C17 from one subunit makes an anti-parallel bridge to C38 of another subunit. Upon dimer formation, the catalytic subunits get released and can phosphorylate 
downstream targets, like PLB and troponin (fig.1.8) (Brennan et al., 2006).

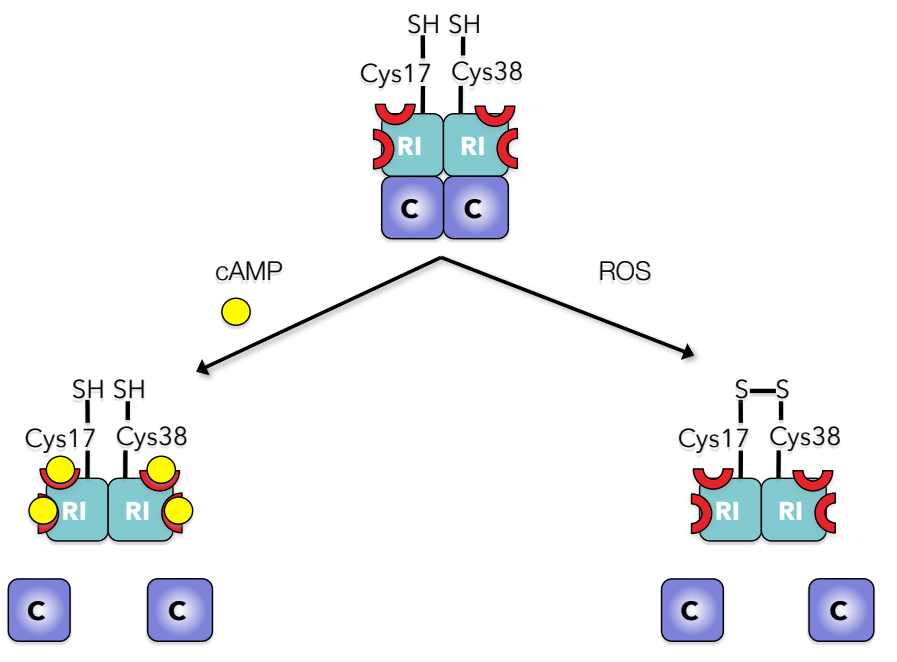

Figure 1.8. Schematic diagram showing activation pathways of type I protein kinase A. PKA can be activated upon binding of cAMP or oxidation of regulatory subunits. When four molecules of cAMP bind to the regulatory subunits, conformational changes happen, which releases the catalytic subunits. Even in the absence of cAMP, a similar phenomenon is observed upon oxidation of cysteine residues of these regulatory subunits.

This RI disulfide formation has been shown to be necessary for cAMPindependent PKA-dependent vasodilation (Burgoyne and Eaton, 2009). Recently PKA RI oxidation has also been shown to mediate vessel growth downstream of growth factor receptor activation. Mice expressing PKA type I, which is unable to undergo oxidant-dependent activation, suffered from impaired angiogenesis in models of hind-limb ischemia and tumor vascularization (Burgoyne et al., 2015). However, the role of oxidant-dependent PKA activation in the heart, specifically its role in the transition from hypertrophy to heart failure, is completely unknown and subject to investigation in the present study (see below).

\subsection{2. $\mathrm{Ca}^{2+} /$ Calmodulin kinase II (CaMKII)}

The stimulation of $\beta$-AR can act via a pathway, other than PKA, which involves the $\mathrm{Ca}^{2+} /$ Calmodulin kinase (CaMKII). CaMKII can be activated by a PKAdependent increase in $\mathrm{Ca}^{2+}$, or a PKA-independent manner via a guanine nucleotide exchange protein directly activated by cAMP (Epac) (Grimm and Brown, 2010). This enzyme is a homo-multimeric serine/threonine kinase and having four isoforms $(\alpha, \beta, \gamma$ and $\delta$ ). Among these, $\delta$ is the predominant isoform in heart. It plays a pivotal role in regulating cardiac performance and remodeling such as cardiomyocyte hypertrophy (Zhu et al., 2000), apoptosis (Zhu et al., 2003), and heart failure (Zhang et al., 2002). In the cell, CaMKII is activated upon binding of $\mathrm{Ca}^{2+}$-bound Calmodulin $\left(\mathrm{Ca}^{2+}-\mathrm{CaM}\right)$ to its regulatory domain. Resulting conformation changes enable the catalytic domain of the enzyme for substrate phos- 
phorylation. It can phosphorylate an array of essential proteins involved in cardiac EC coupling and $\mathrm{Ca}^{2+}$ handling. Such as the sarcoplasmic/endoplasmic reticulum $\mathrm{Ca}^{2+-A T P a s e}$ (SERCA2a) at S38 (Toyofuku et al., 1994) and its regulator, Phospholamban (PLB) at T17 (Le Peuch et al., 1979; Simmerman et al., 1986), ryanodine receptor (RyR2) at S2814/2415 (Wehrens et al., 2004) and sarcolemmal L-type $\mathrm{Ca}^{2+}$ channels (LTCC) (Grueter et al., 2006; Koval et al., 2010). Phosphorylation of $\mathrm{Na}_{\mathrm{v}} 1.5$ (Wagner, Dybkova et al. 2006, Hund, Koval et al. 2010) and RyR2 causes increased $\mathrm{Na}^{+}$entry as well as $\mathrm{SR} \mathrm{Ca}^{2+}$ depletion leading to $\mathrm{Na}^{+}$and $\mathrm{Ca}^{2+}$ overload (Ai et al., 2005; Maier et al., 2003; Wagner et al., 2011; Zhang et al., 2003). PLB phosphorylation resulting in an increased SERCA2a function and depending on the particular condition may counterbalance $\mathrm{SR} \mathrm{Ca}^{2+}$ depletion to some extent, (Davis et al., 1983; Sag et al., 2009; Simmerman et al., 1986).

In addition to substrate phosphorylation, CaMKII auto-phosphorylates itself at T286/T287, depending on species, which prevents re-association of catalytic and regulatory domains. This auto-phosphorylation slows the enzyme inactivation kinetics as well as ensures maximal kinetic activity by acting as a memory of activation. Interestingly, it has been shown that ROS can modulate CaMKII function in a similar way by oxidizing methionine 281/282 (M281/282) in the regulatory domain (Erickson et al., 2008). Upon oxidation of M281/282, the catalytic subunit cannot re-associate with the regulatory domain thus can continue its enzymatic activity.

It has been reported that treatment of cardiomyocytes with $\mathrm{H}_{2} \mathrm{O}_{2}$ increases the incidence of pro-arrhythmogenic $\mathrm{Ca}^{2+}$ sparks and pro-hypertrophic $\mathrm{Ca}^{2+}$ overload downstream of CaMKII activation (Wagner et al., 2011). In addition, AngIIdependent activation of NOX2, in vitro and in vivo, increased M281/282 oxidation and induced apoptosis, which was lost in M281/282V CaMKII knock-in (KI) mice (Erickson et al., 2008). Interestingly, Methionine sulfoxide reductase A, a reductant, can reduce CaMKII and stop these oxidized-CaMKII mediated phosphorylations. 


\subsubsection{Protein kinase C (PKC)}

$\mathrm{PKC}$ is a family of serine/threonine kinases, which have at least 12 isozymes and can control EC coupling at several stages. The activation mechanism varies among the subtypes. For example, isoform $\alpha, \beta$ I, $\beta$ II, and $\gamma$ are activated by $\mathrm{Ca}^{2+}$ and diacylglycerol (DAG); in contrast, $\delta, \varepsilon, \theta, \eta, \zeta$ and $\lambda$ are activated by distinct lipids (Dorn and Force, 2005). Moreover, a report has shown duel role of oxidants on PKC, where mild oxidation can reduce $\mathrm{Ca}^{2+} /$ phospholipid-dependent activation followed by an immediate activation, independent of $\mathrm{Ca}^{2+}$ and phospholipids (Gopalakrishna and Anderson, 1989). Upon excessive oxidant production, these intermediate effects diminished and the enzyme completely inactivated (Gopalakrishna and Anderson, 1987). The amount of oxidants along with many isoforms of PKC, make it difficult to find out the exact role of oxidized PKC in pathophysiologic settings. However, a recent report has shown that in stress conditions, like, myocardial infarction can increase the expression of several isoform of PKC, which ultimately causes cardiac dysfunction (Wang et al., 2003).

\subsection{Oxidants}

Oxidants are reactive molecules; produced under different physiological and pathophysiological settings. These are mainly reactive molecules containing oxygen (reactive oxygen species, ROS) in either their radical or non-radical forms. These oxidants are unstable molecules and can attack any biological molecules including protein (ex. fragmentation or aggregation), lipid (ex. lipid peroxidation) and DNA (destruction of sugar or base, strand breakage) (Pham-Huy et al., 2008). Oxidants can be generated enzymatically or by electrons that leak from the electron transport chain and are transferred to $\mathrm{O}_{2}$ to form the superoxide radical $\left(\mathrm{O}_{2}{ }^{*}\right)\left(\mathrm{O}_{2}+\mathrm{e}^{-} \rightarrow \mathrm{O}_{2}{ }^{*}\right)$ (Poole and Nelson, 2008). The addition of a second electron to $\mathrm{O}_{2}{ }^{{ }^{*}}$ can occur spontaneously or can be catalyzed by superoxide dismutase (SOD) to yield the more stable, and less reactive, signaling molecule hydrogen peroxide $\left(\mathrm{H}_{2} \mathrm{O}_{2}\right)\left(2 \mathrm{H}^{+}+2 \mathrm{O}_{2}{ }^{*-} \rightarrow \mathrm{H}_{2} \mathrm{O}_{2}+\mathrm{O}_{2}\right)$. Further electron addition to $\mathrm{H}_{2} \mathrm{O}_{2}$ via the Fenton reaction, where $\mathrm{Fe}^{2+}$ donates an electron to become $\mathrm{Fe}^{3+}$, produces the hydroxyl radical $\left(\mathrm{OH}^{*}\right)$, a strongly oxidizing molecule, which is highly reactive and is not considered as a viable signaling molecule (Shao et al., 2012). There are many other ROS, including the peroxyl radical (ROO*), hydrop- 
eroxyl radical $\left(\mathrm{HOO}^{*}\right)$, hydroxyl ion $\left(\mathrm{OH}^{-}\right)$and the hypochlorite ion $\left(\mathrm{OCl}^{-}\right)$. Moreover, oxidative signaling also involve nitrogen-containing molecules including the nitroxyl ion ( $\left.\mathrm{NO}^{-}\right)$, peroxynitrite ( $\left.\mathrm{ONOO}^{-}\right)$, dinitrogen trioxide $\left(\mathrm{N}_{2} \mathrm{O}_{3}\right)$, nitroxyl radical $\left(\mathrm{NO}^{*}\right)$, nitrosonium ion $\left(\mathrm{NO}^{+}\right)$, nitronium ion $\left(\mathrm{NO}_{2}{ }^{+}\right)$, nitrite ion $\left(\mathrm{NO}_{2}^{-}\right)$, and nitrate ion $\left(\mathrm{NO}_{3}^{-}\right)$(Pacher et al., 2007).

The production and quenching of oxidants are tightly maintained to control its role, as any deregulation may cause severe damage to the body (Maxwell, 1995). Interestingly, either excess use of drugs, which can produce oxidants (paracetamol associated hepatotoxicity), or administrations of high doses of antioxidants (vitamin E associated membrane damage) both are detrimental for the body (Maxwell, 1995) (Bjelakovic et al., 2007; Griendling and FitzGerald, 2003; Hennekens et al., 1996; Melov, 2002). The underlying mechanism may involve electron transfer reaction between free radicals and target molecules. During this transfer, the unpaired highly reactive electron of the free radical collects one electron from a non-radical target molecule. The target molecule becomes oxidized (electron donor), and the free radical is reduced (electron acceptor). If this reaction results in the formation of an unstable oxidized target molecule, this can now incorporate a highly reactive free electron to a new substrate. Thus a new radical is formed. If antioxidant follows this chain, then reactive free radicals can be produced continuously, which can ultimately lead to cellular damage. This is one of the mechanisms by which excess antioxidants may cause harm to the cell. For example, ROS scavengers, N-acetylcysteine (NAC), can undergo autooxidation before treatment, which effectively rendering them as oxidants (Chan et al., 2001) and may exert an opposite effect to that one intended (Kornfeld et al., 2015). Moreover, the fact that antioxidants are toxic is further confirmed by the finding that overexpression of the antioxidant, glutathione peroxidase (Gpx1) like protein GTPX-1, suppressed differentiation of multipotent hematopoietic progenitors in Drosophila (Owusu-Ansah and Banerjee, 2009).

On the other hand, if this reaction results in the formation of an oxidized target molecule with stable electrons, the chain terminates. The energy of the free radical is scavenged, and no further oxidation reaction is possible. The latter is the 
case for reaction of oxidants with physiologic antioxidant concentration (Maxwell, 1995).

Interestingly, the role of oxidants in cellular signaling is further evident from $\mathrm{H}_{2} \mathrm{O}_{2} . \mathrm{H}_{2} \mathrm{O}_{2}$ is produced from the highly reactive oxidant superoxide $\left(\mathrm{O}_{2}{ }^{*}\right)$ via a reaction catalyzed by superoxide dismutase (SOD). However, due to its high reactivity $\mathrm{O}_{2}{ }^{*}$ - has very limited diffusion distance, in the range of only a few nanometers, and scavenged rapidly. $\mathrm{H}_{2} \mathrm{O}_{2}$, on the other hand, is less reactive, small, and neutral. Besides, it is highly diffusible and able to cross cellular membranes to act as a signaling molecule. Thus, $\mathrm{H}_{2} \mathrm{O}_{2}$ can execute multiple physiological roles within the cell (Burgoyne et al., 2012; Wagner et al., 2013). On the other hand, at high concentrations, it may also be cytotoxic to the cell (Poole and Nelson, 2008). At high concentrations, $\mathrm{H}_{2} \mathrm{O}_{2}$ form more reactive hydroxyl radical $\left(\mathrm{OH}^{*}\right.$, via Haber-Weiss reaction or via Fenton reaction) by reaction with $\mathrm{H}_{2} \mathrm{O}$ or peroxynitrite ( $\mathrm{ONOO}^{-}$, by reaction with $\mathrm{NO}$ ). These products can also play a role in cellular signaling. Interestingly, $\mathrm{H}_{2} \mathrm{O}_{2}$-mediated oxidation of cysteine in proteins is reversible, like other regulatory posttranslational modifications such as phosphorylation. This duality of oxidants, involvement in both cell signaling and cell damage, is also observed in the heart. For example, oxidants are involved in physiological regulation of excitation-contraction coupling, the regulation of vascular tone, but also in the stress responses to hypoxia, ischemia, inflammation leading to arrhythmias and contractile dysfunction (Burgoyne et al., 2012; Madamanchi et al., 2005).

\subsubsection{Sources of oxidants}

There are several sources for oxidants within the cell. Among them, mitochondria are the primary source of cellular ROS. Under normal circumstances, $\mathrm{O}_{2}$ is the terminal electron acceptor at complex IV of the electron transport chain (ETC) where it is reduced to form $\mathrm{H}_{2} \mathrm{O}$. However, electrons can 'leak' at complex I and III where they can be transferred to $\mathrm{O}_{2}$ to generate $\mathrm{O}_{2}{ }^{*}$ - (Jastroch et al., 2010). In addition to ETC, monoamine oxidase (MAO) is located on the outer membrane of the mitochondria. It catalyzes the oxidative deamination of adrenaline (A), noradrenaline (NA), serotonin (5-HT) and other related monoamines to produce 


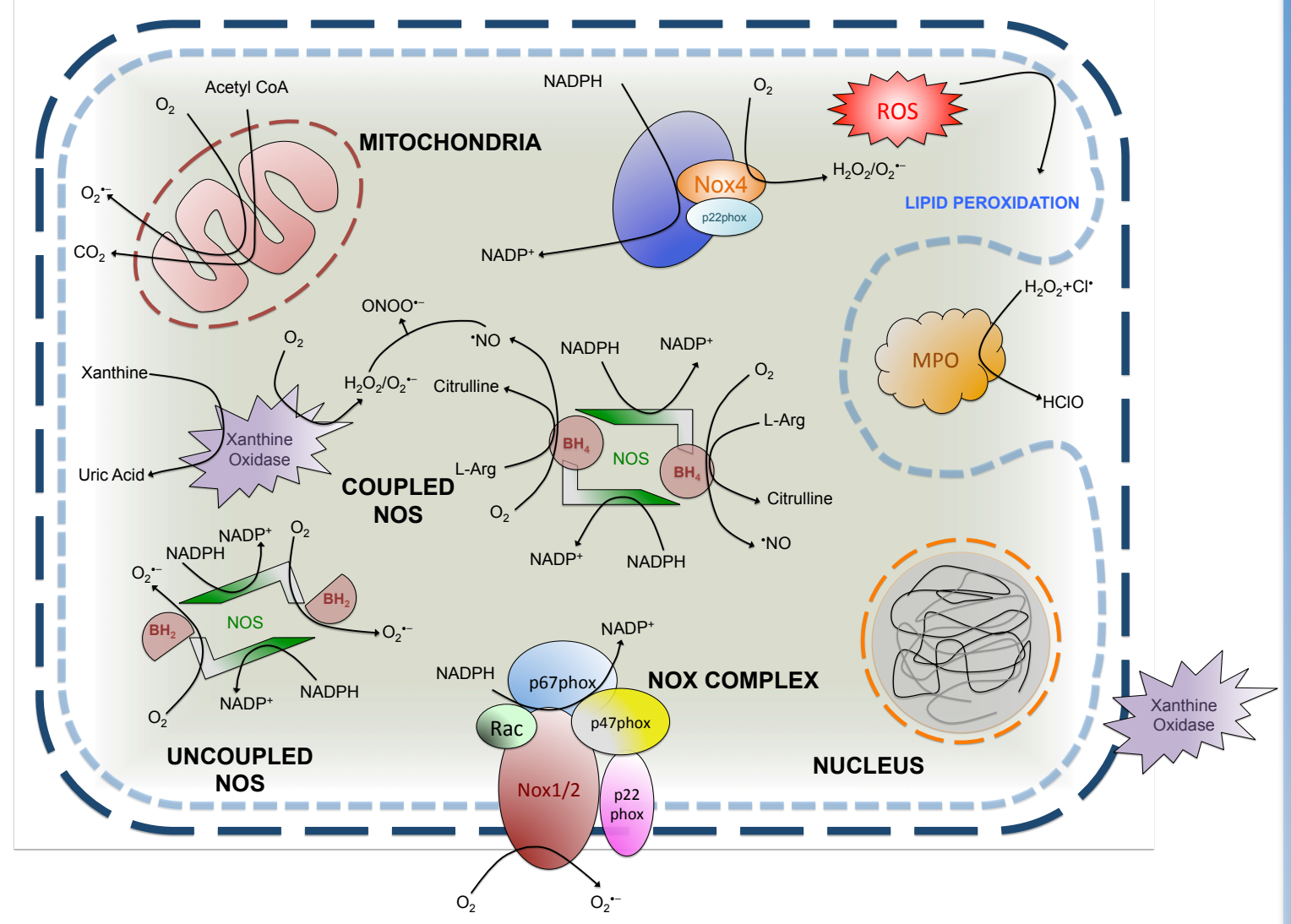

Figure 1.9. Sources of ROS generation. Mitochondrial electron transport chain leak electrons and pass directly to oxygen, thus generating superoxide radical $\left(\mathrm{O}_{2}{ }^{*}\right)$. This can also be formed by xanthine oxidase (XO), which catalyzes the oxidation of hypoxanthine and xanthine. In addition NOX enzymes utilize NADPH as an electron donor and catalyze the transfer of electrons to molecular oxygen to generate $\mathrm{O}_{2}{ }^{{ }^{*}}$ and/or $\mathrm{H}_{2} \mathrm{O}_{2}$. Nitric Oxide synthases (NOS) generate ${ }^{*} \mathrm{NO}$ and Lcitrulline from arginine and $\mathrm{O}_{2}$. In the absence of tetrahydrobiopterin (BH4) or L-arginine, uncoupled NOS enzymes resulting in the production of $\mathrm{O}_{2}{ }^{*-}$ rather than ${ }^{*} \mathrm{NO}$. In addition, activated monocytes secrete heme-containing myeloperoxidase(MPO), that uses $\mathrm{H}_{2} \mathrm{O}_{2}$ as a substrate to generate products, like hypochlorous acid (HOCI) that can oxidize lipids and proteins. Within cell membranes, ROS can trigger lipid peroxidation, a self-propagating chain reaction that can result in significant tissue damage.

$\mathrm{H}_{2} \mathrm{O}_{2}$ as a by-product (Tipton et al., 2004). It has been reported that mice lacking mitochondrial manganese superoxide dismutase (SOD2) die within 10 days with dilated cardiomyopathy, implicating the role of mitochondrial ROS in disease condition (Li et al., 1995). Similarly, SS31, (Bendavia), shows a cardioprotective effect in response to IR damage in multiple animal models of myocardial infarction (MI) and heart failure (HF), including in mice, rats, guinea pigs, rabbits, and sheep. This is a four amino acid synthetic peptide (phenylalanine-d-argininephenylalanine-lysine), selectively reaches the inner mitochondrial membrane, scavenges ROS through a dimethyltyrosine group and reduces mitochondrial ROS production (Cho et al., 2007; Dai et al., 2013; Kloner et al., 2012). Moreover, Overexpression of catalase in the mitochondria (mCat) also reduces mitochon- 
drial oxidative damage, increases the lifespan of mice, and protects against angiotensin II-induced cardiac hypertrophy, fibrosis, and HF (Dai et al., 2011; Schriner et al., 2005). Thus, implicating the role of mitochondrial ROS in disease conditions.

Another important source of oxidant is the NADPH oxidase (NOX). Unlike the mitochondria, where oxidants are created as a byproduct, NOXes are enzymes specifically designed to produce ROS. They mostly generate $\mathrm{O}_{2}{ }^{*}$ by transferring electrons from NADPH to flavin and ultimately to molecular $\mathrm{O}_{2}\left(2 \mathrm{O}_{2}+\mathrm{NADPH} \rightarrow\right.$ $2 \mathrm{O}_{2}{ }^{*}{ }^{-}+\mathrm{H}^{+}+\mathrm{NADP}^{+}$) (Burgoyne et al., 2012). The NOX is a multi-subunit enzyme complex composed of membrane spanning flavocytochrome $b_{558}$ (consisting of flavin, the haem-binding gp91phox, and p22phox), the cytosolic regulators (p47phox, p67phox, p40phox) as well as the small GTPase (Rac 1, 2) (fig.1.9) (Ago et al., 2004).

Distinct combinations of these subunits make different NOX isoforms named as NOX1, NOX2, NOX3, NOX4, NOX5, dual oxidase 1 (Duox1), and Duox2, which are expressed selectively depending on tissue type (Zhang et al., 2013). For example, NOX1, NOX2, NOX4, and NOX5 are expressed in the cardiovascular system (with NOX5 only found in higher mammals). Within the cardiovascular system, both NOX2 and NOX4 are expressed in endothelium and cardiomyocytes. NOX2 is located predominantly on the plasma membrane and activated by hormones, growth factors and cytokines (angiotensin II, insulin, platelet-derived growth factor (PDGF) and tumor necrosis factor $\alpha(T N F \alpha)$ ). In contrast, NOX4 is a constitutively active intracellular enzyme, where its activity depends on its abundance (Lassegue et al., 2012). NOX1 is predominately located in vascular smooth muscle cells (VSMC) and Nox5 is in VSMC, fibroblasts and endothelial cells (Zhang et al., 2013).

Compared to other NOX enzymes, which mainly produced superoxide $\left(\mathrm{O}_{2}{ }^{{ }^{-}}\right)$, NOX4 can produce $\mathrm{H}_{2} \mathrm{O}_{2}$ with its special E-loop structure, which differs significantly from that of NOX2. E-loop contains a highly conserved histidine moiety that may hinder superoxide egress and provide a source of protons, allowing 
dismutation to form $\mathrm{H}_{2} \mathrm{O}_{2}$ (Takac et al., 2011). This may imply distinct roles of NOX isozymes inside the cell. This is further revealed in NO signaling, where $\mathrm{O}_{2}{ }^{*}$ produces peroxynitrite and disrupt NO signaling. However, $\mathrm{H}_{2} \mathrm{O}_{2}$ does not undergo this reaction and instead may be even enhanced NOS activity and signaling (Cai et al., 2001).

Beside NOX and mitochondria, cytosolic xanthine oxidase (XO) is another important source of oxidants. Xanthine oxidase involves in purine catabolism to produce uric acid from hypoxanthine and xanthine. This reaction reduces molecular oxygen to yield superoxide (Berry and Hare, 2004). Studies have shown that XO expression, as well as activity, is increased in cardiomyocytes isolated from failing hearts (Cappola et al., 2001; Ekelund et al., 1999; Saavedra et al., 2002; Stull et al., 2004). In contrast, in rodent or canine heart failure models, inhibition of XO activity with allopurinol or oxypurinol improved myocardial function, decreased myocardial oxygen consumption and ameliorated myocardial contractility (Ekelund et al., 1999). Moreover, ascorbate treatment resulted in the same benefits that were seen with allopurinol (Saavedra et al., 2002). These confirm that the effects resulted from inhibition of XO are generated from ROS. Moreover, it was reported that prolonged inhibition of XO with allopurinol in rodent models of post-infarction heart failure could improve contractile function, and prevent ventricular remodeling (Cappola et al., 2001; Mellin et al., 2005; Stull et al., 2004). These effects are, may be, the results from allopurinol-mediated XO inhibition and a resultant decrease in ROS levels.

Besides, cellular nitric oxide synthase (NOS) produces nitric oxide (NO) in an uncoupling reaction from L-arginine, which is also an important cellular oxidant (Wagner et al., 2013). There are three main isoforms of NOS: neuronal NOS (nNOS), endothelial NOS (eNOS), and inducible NOS (iNOS). A variety of cell types express these isoforms and sometimes expressing even more than one isoform. The cardioprotective roles of NOS enzyme are revealed by the administration of intravenous non-selective NOS inhibitors, which in experimental coxsackie B3 viral myocarditis, increased cardiac damage and mortality (Hiraoka et al., 1996; Lowenstein et al., 1996). However, in heart, NO inhibits L-type $\mathrm{Ca}^{2+}$ chan- 
nels (Mery et al., 1993) but stimulates sarcoplasmic reticulum (SR) $\mathrm{Ca}^{2+}$ release (Eu et al., 2000; Petroff et al., 2001; Xu et al., 1998a), leading to variable effects on myocardial contractility. Studies have also shown that heart failure is associated with induced iNOS gene expression in the ventricular myocardium. Moreover, iNOS protein is present in ventricular cardiomyocytes from patients with end-stage heart failure secondary to dilated cardiomyopathy, ischemic heart disease, or valvular heart disease (Haywood et al., 1996). Thus, it is still under investigation to find out the exact role of NOS expression in cardiac disease conditions.

\subsubsection{How does the cell control oxidant detoxification?}

Cardiomyocytes harbor strong antioxidant system to avoid detrimental effects of oxidants. This system contains enzymes, which are located in different subcellular compartments. Superoxide dismutase (SOD), for instance, rapidly converts $\mathrm{O}_{2}{ }^{*}$ - into $\mathrm{H}_{2} \mathrm{O}_{2}$. Due to SODs high abundance and rapid catalytic rate, $\mathrm{O}_{2}{ }^{*}$ - diffusion is very limited. There are three isoforms of SOD; copper-zinc SOD (SOD1) located in the cytosol, manganese SOD (SOD2) located in the mitochondria, and copperzinc SOD (SOD3) located in the extracellular space (Faraci and Didion, 2004). In addition, several $\mathrm{H}_{2} \mathrm{O}_{2}$-neutralizing peroxidases exist as antioxidant including catalase, glutathione peroxidase (Gpx1), and peroxiredoxin (Prx). Catalase is located in cytosolic peroxisomes, where it produces $\mathrm{H}_{2} \mathrm{O}$ and $\mathrm{O}_{2}$ as a by-product from $\mathrm{H}_{2} \mathrm{O}_{2}$ (Mates et al., 1999). In contrast, Gpx1 is localized in the cytosol, which catalyzes the oxidation of glutathione (GSH) to detoxify $\mathrm{H}_{2} \mathrm{O}_{2}$. As a result, the thiol group of GSH is oxidized to form an intermolecular disulfide bridge with another GSH molecule to generate GSSG. The oxidation of GSH prevents the unintended oxidation of amino acid residues of proteins and enzymes, which is named as scavenging (Mates et al., 1999). It is noted that GSH is the most abundant intrinsic radical scavenger inside the cell. Importantly, GSH and GSSG levels are strictly controlled by the glutathione reductase (Gsr), which uses NADPH to reduce GSSG into GSH (fig.1.10). Thus, the level of GSH to GSSG in different subcellular compartments can be utilized as a measure of the redox potential of that specific compartment (Aller et al., 2013), which may indicate the likelihood of proteins to become oxidized or reduced in distinct compartments. 


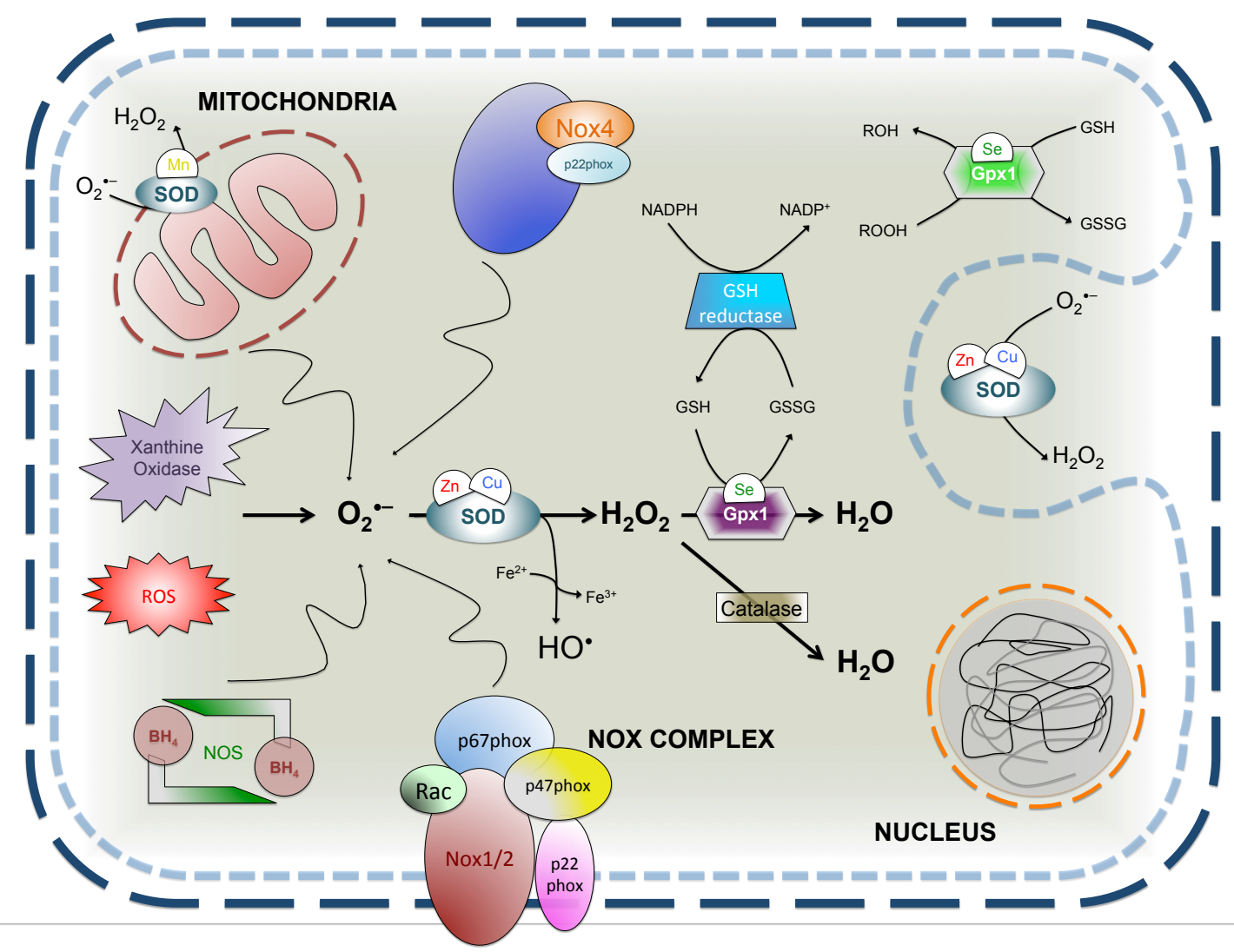

Figure 1.10. Schematic figure representing the major antioxidant system inside cells. ROS are usually generated by electron transport chain (ETC) leakage and NOX family proteins, which are neutralized by antioxidants including SOD family proteins, catalase, GSH and Trx systems. (modified from Lipid Peroxidation and Antioxidants in Arterial Hypertension. Teresa Sousa, Joana Afonso, António Albino-Teixeira and Félix Carvalho. Lipid Peroxidation, August 2012, doi: 10.5772/50346.)

Peroxiredoxin (Prx) is another cytosolic enzyme that catalyzes the detoxification of $\mathrm{H}_{2} \mathrm{O}_{2}$ by oxidation of an intrinsic thiol residue (Wood et al., 2003). Therefore, it is not only an enzyme involved in detoxification of oxidant but also a scavenger itself. Oxidized Prx is then reduced by thioredoxin (Trx) via a thiol-disulfide exchange (R-S-S-R + R'-S- $\rightarrow$ R-S- + R'-S-S-R), which results in the oxidation of Trx but restores the catalytic activity of Prx. Oxidized Trx is then reduced by thioredoxin reductase (TrxR) using NADPH and FAD (Holmgren, 1995). Thus, it is implicated that cell maintains a chain of oxidoreductase reactions to stepwise and gently reduce the large oxidative potential of free radicals.

Beside these active enzymatic detoxification strategies, cardiomyocytes also use non-enzymatic scavengers like vitamins (A, C and E), flavonoids, uric acid, bilirubin, albumin, ceruloplasmin, transferrin and others to control intracellular redox potential (Chow et al., 1999; Mari et al., 2009; Maxwell, 1995; Padayatty et al., 
2003).

\subsubsection{Oxidative modifications of functionally important myocardial pro- teins and contractile dysfunction}

It is known that $\mathrm{Ca}^{2+}$-handling proteins are important substrates for oxidation. (Maxwell, 1995). The mechanisms are incompletely understood but may involve direct oxidation of the protein or upstream serine/threonine kinases or phosphatases. The latter indirectly influences the function of $\mathrm{Ca}^{2+}$-handling proteins by changing their phosphorylation status (Brennan et al., 2006).

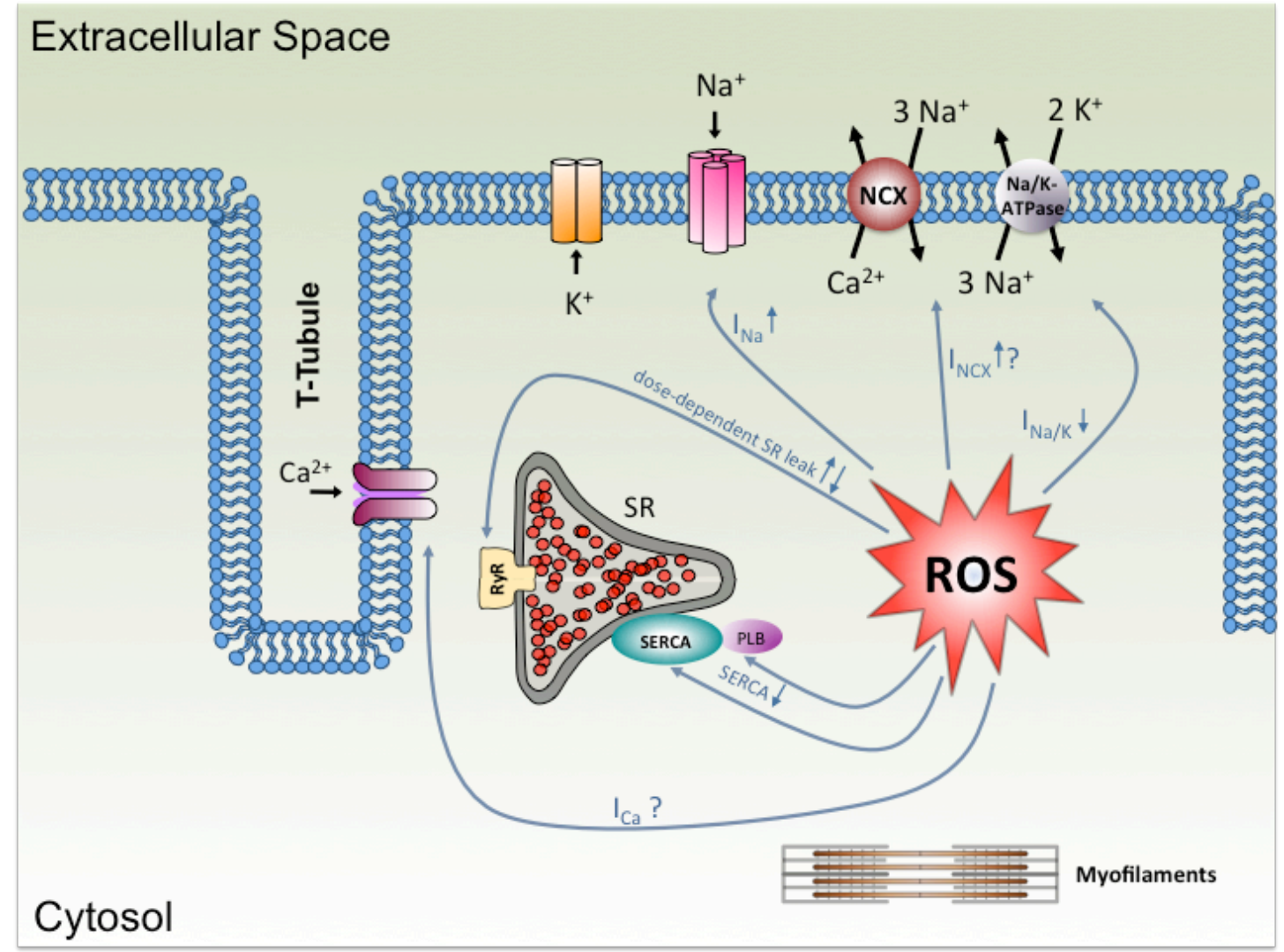

Figure 1.11. Schematic diagram showing role of ROS in EC coupling. Upon production, ROS can modify several targets of EC coupling for example LTCC, RyR2, cardiac $\mathrm{Na}^{+}$channel, SERCA2a, PLB directly or indirectly to modulate their functions. (modified from Wagner et al., 2013)

Interestingly, this direct oxidation may happen to proteins' reactive cysteine thiols. Most of the cysteine thiols are unreactive at physiological $\mathrm{pH}$ because of their high acid dissociation constant $\left(\mathrm{p} K_{\mathrm{a}}\right)$ of $\sim 8.5$. However, their reactivity not only depends on $\mathrm{pH}$ but also on the surrounding tertiary structure of the protein. If basic amino acid residues such as arginine, histidine or lysine are in close proximity to a cysteine, its $\mathrm{p} K_{\mathrm{a}}$ can be lower rendering it more likely to be reactive at physiological $\mathrm{pH}$. 
Thus within a protein, very few cysteine thiols are deprotonated (-RS-) and could be the substrate for oxidation by free radicals (Burgoyne et al., 2012). It has been suggested that ROS can decrease LTCC (Favero et al., 1995; Gill et al., 1995; Goldhaber et al., 1989; Hu et al., 1997; Lacampagne et al., 1995), SERCA2a (Kukreja et al., 1988; Morris and Sulakhe, 1997; Scherer and Deamer, 1986; Xu et al., 1997) and $\mathrm{Na}^{+} / \mathrm{K}^{+}$ATPase (Kim and Akera, 1987; Kukreja et al., 1990; Shattock and Matsuura, 1993; Xie et al., 1990) function upon oxidation. In contrast, ROS can increase RyR2 (Abramson and Salama, 1989; Boraso and Williams, 1994; Xu et al., 1998a) and NCX (Goldhaber, 1996; Kato and Kako, 1988; Reeves et al., 1986; Santacruz-Toloza et al., 2000) activity (fig.1.11).

\subsubsection{Redox-dependent gene transcription}

During each cycle of excitation-contraction coupling, $\mathrm{Ca}^{2+}$ diffuses into different compartments. Diffusion of $\mathrm{Ca}^{2+}$ into the nucleus can activate gene expression, which is known as excitation-transcription coupling (ET coupling). These pathways are rather slow and allow the cardiomyocyte to adapt to chronic stress that ultimately causes expression of different regulatory and effector proteins (Bers, 2001). In addition to $\mathrm{Ca}^{2+}$, oxidants are also mentioned as a mediator of gene expression. For example, oxidation of cysteine residues in Kelch-like ECHassociated protein 1 (Keap1) changes its conformation, thus releasing associated nuclear factor-erythroid-2-related factor 2 (Nrf2) and facilitating its translocation to the eukaryotic nucleus. This free Nrf2 then binds to the antioxidant response element (ARE) located in the promoter region of genes to up-regulate transcription of various proteins (Gorrini et al., 2013; Soriano et al., 2009). Moreover, $\mathrm{H}_{2} \mathrm{O}_{2}$ signaling is also involved in gene regulation. Vascular endothelial growth factor (VEGF) increases cellular $\mathrm{H}_{2} \mathrm{O}_{2}$ downstream of VEGF receptor 2 and NOX4 activation. There it directly activates extracellular signal-regulated kinase (ERK1/2), a well-established mediator of growth factor signaling that results in the up-regulation of genes involved in endothelial cell proliferation (Ushio-Fukai, 2006).

\subsubsection{Oxidants can increase the propensity for cardiac arrhythmias}

Recently it has been reported that stretch-induced NOX2 activation potentiates 
$\mathrm{Ca}^{2+}$ release. This process involved RyR2-mediated increase in $\mathrm{Ca}^{2+}$ spark in the cytosol, which can ultimately lead to arrhythmia (Prosser et al., 2011). Inhibition of direct oxidative activation of RyR2 may give protection against arrhythmia. Evidence showed that nNOS- (which is colocalized to RyR2) mediated RyR2 Snitrosylation could protect XO-dependent thiol oxidation and heart diseases. Similar results showed, AngII-mediated oxidative hyper-phosphorylation of RyR2 could lead to aberrant $\mathrm{Ca}^{2+}$ release and arrhythmogenic waves (Prosser et al., 2011).

Other reports have shown that $\mathrm{H}_{2} \mathrm{O}_{2}$-induced oxidation of CaMKII causes $\mathrm{Na}^{+}$ overload (Wagner et al., 2011). As mentioned, this $\mathrm{Na}^{+}$overload can activate NCX to act in reverse mode and increase cytosolic $\mathrm{Ca}^{2+}$ concentration. This increase in $\mathrm{Ca}^{2+}$ concentration may initiate arrhythmia inside the cell. Moreover, Song et al. showed that oxidized CaMKII could modulate LTCC and increase the peak $\mathrm{I}_{\mathrm{Ca}}$ which is pro-arrhythmogenic to the cell (fig.1.12) (Song et al., 2010).

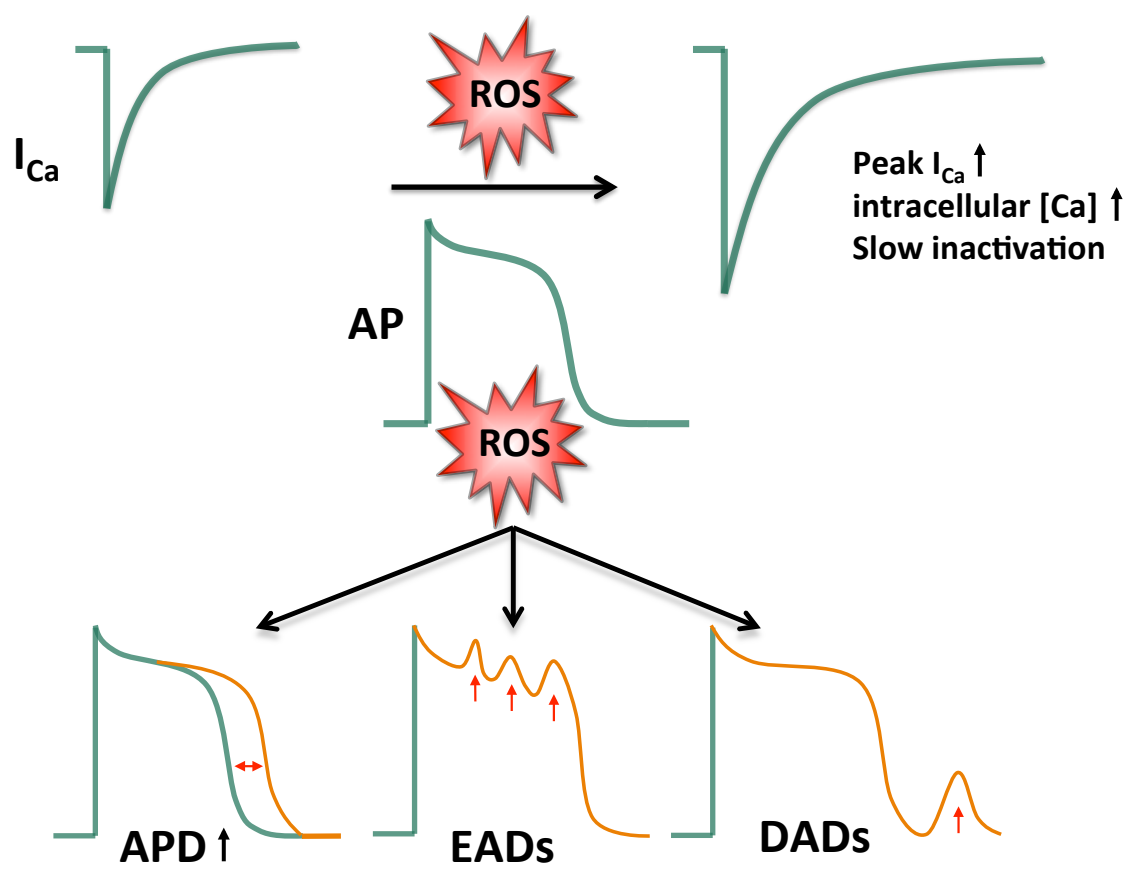

Figure 1.12. Reactive oxygen species (ROS) induces membrane excitability and arrhythmia in cardiomyocytes via modulating calcium current. Intracellular $\mathrm{Ca}^{2+}$ concentration is increased by ROS-mediated change in peak $\mathrm{I}_{\mathrm{Ca}}$. This leads to prolongation of the action potential duration, early afterdepolarizations (EADs), and delayed afterdepolarizations (DADs).

\subsubsection{Oxidants promote hypertrophy}

It has been shown that pharmacological inhibition or genetic deletion of mito- 
chondrial MAO-A can reduce ROS and apoptosis. Thus, improve LV dilation and contractile dysfunction in pressure overload-induced mouse model (Kaludercic et al., 2010). Moreover, chronic AngII infusion for four weeks or transgenic mice with cardiomyocyte-specific $\mathrm{G}_{\mathrm{q}}$ overexpression is responsible for mitochondrial ROS production. This ROS lead to subsequent protein oxidation, mitochondrial DNA damage and ultimately leads to heart failure. In accordance, mice with mitochondrial-targeted catalase overexpression showed significant protection (Dai et al., 2011). Thus, implicating the role of oxidants in cardiac hypertrophy. NOX2 is also involved in AngII-induced hypertrophy, which is further confirmed by NOX2 knockout mice, where AngII-mediated hypertrophy was absent (Hingtgen et al., 2006) (Bendall et al., 2002).

\subsubsection{Oxidants induce apoptosis of cardiomyocytes}

Cell death is the ultimate pathway for cellular defense, which as a consequence leads to severe cardiac injury or heart failure (Dorn, 2009). Oxidants can directly activate this apoptotic pathway via a $\mathrm{Ca}^{2+}$-dependent opening of the mitochondrial permeability transition pore (MPTP) or indirectly by promoting B-cell lymphoma 2 (Bcl-2) associated X protein/death promoter (Bax/Bad) translocation to mitochondria. Bax/Bad translocation is mostly mediated by p53, which is activated by oxidant-induced DNA damage. Upon activation, p53 induces Mouse double minute 2 homolog (MDM2, an E3 ubiquitin ligase) to degrade an apoptosis repressor that interacts with Bax to inhibit apoptosis (Foo et al., 2007). Evidence has been shown that mice deficient of this repressor can develop more rapid pressure overload-induced heart failure and larger infarct size after ischemia-reperfusion (Donath et al., 2006). In addition, oxidants can also activate apoptosis signaling kinase 1 (ASK-1), p38 Mitogen-activated protein kinases and c-Jun N-terminal kinase that lead to activation of the mitochondrial death pathway (Remondino et al., 2003). This is in agreement with reports showing that either pressure overload (Yamaguchi et al., 2003) or MI (Erickson et al., 2008) or AngII-stimulated NOX2-derived ROS is involved in the activation of ASK-1 and apoptosis (Hirotani et al., 2002). 


\subsubsection{ROS production during ischemia-reperfusion and heart failure}

During acute coronary syndrome, insufficient coronary blood flow results in ischemia and the restoration of blood flow cause reperfusion. At the cellular level ischemia leads to hypoxia and reperfusion is associated with re-oxygenation (Fitzpatrick and Karmazyn, 1984). It has been shown that while free radicals are generated during hypoxia, vast amount of oxidants are produced during reoxygenation by the respiratory chain and other enzymes like xanthine oxidase and nitric oxide synthases (Puett et al., 1987; Shlafer et al., 1987; Zweier and Talukder, 2006), which can ultimately damage myocardium. This damage causes functional alterations of heart that includes depressed contractile function, decreased coronary flow, and altered vascular reactivity.

Moreover, during EC coupling, physiologic contraction itself can increase a local and transient rise in oxidant concentration (Prosser et al., 2011). However, extensive, persistent and ubiquitous increase of these oxidants can lead to contractile dysfunction and heart failure (Ide et al., 2000; Ide et al., 1999; Kim et al., 2006; Mulrooney et al., 2009; Sag et al., 2011; Sag et al., 2013; Timolati et al., 2006; Tokarska-Schlattner et al., 2006). Evidence show NOX2 expression levels are increased in the heart early after acute myocardial infarction (MI) in both humans (Bers, 2008) and animal models. (Bers et al., 2003) Part of this increase in NOX2 expression is due to inflammation associated migration of neutrophils and mononuclear cells into the heart (Ambrosio et al., 1986; Lefer et al., 1990; Przyklenk and Kloner, 1989; Rowe et al., 1984; Werns et al., 1985). In the later phase of MI, progressive hypertrophy and fibrosis occur in the remote myocardium, which leads to LV dilation and functional deterioration. Interestingly, both p47phox-/- and NOX2-null mice have reduced adverse post-MI remodeling during four weeks time period, showing that NOX2 is involved even in the later phase of this process (Looi et al., 2008) (Doerries et al., 2007). Moreover, inhibition of kinases, which are downstream to ROS, can also provide beneficial effects. For example, MI-mediated detrimental effects can be blocked by CaMKII inhibitors or by antioxidants (Erickson et al., 2008; Koval et al., 2012; Purohit et al., 2013). 


\subsection{Angiotensin II-mediated signaling in cardiomyocytes}

The renin-angiotensin system is involved in the pathogenesis of different cardiac diseases (Brilla et al., 1990; Hanatani et al., 1995; Weber et al., 1993). Upon its production from the juxtaglomerular cells of the kidney, renin is released primarily into the blood, where it proteolytically cleaves angiotensinogen (produced in the liver) to form the decapeptide Angiotensin I (AngI). AngI can be subsequently cleaved by angiotensin converting enzyme (ACE) and generates the octapeptide AngII within the pulmonary circulation (Campbell, 1987; Johnston, 1992). In addition to the circulating RAS, many other tissues can also synthesize AngII in the presence of angiotensinogen, renin, and ACE, which includes heart, vasculature, kidney, and brain. Thus, explaining the paracrine and intracrine effects of AngII (Campbell, 1987; Johnston, 1992; Phillips et al., 1993; Vinson et al., 1995).

The main effector of renin-angiotensin system pathway is angiotensin II (AngII). It can initiate vasoconstriction and hypertrophic responses upon binding to its receptors, named $\mathrm{AT}_{1}$ and $\mathrm{AT}_{2}$. Both of these are G-protein-coupled seven transmembrane receptors (Nicholls et al., 2001). While it has been reported that $\mathrm{AT}_{1}$ is responsible for growth and proliferation, $\mathrm{AT}_{2}$ exerts the opposite effects (Wen et al., 2012). More recently, $\mathrm{AT}_{1}$ antagonists were found to be effective at repressing cardiac hypertrophy in hypertensive patients (Thurmann et al., 1998). However, although enhanced $\mathrm{AT}_{2}$ levels may be compensatory by decreasing the cardiac output and normalizing blood pressure, further increase in $\mathrm{AT}_{2}$ may have a detrimental effect on the heart leading to hypertrophy (Harada et al., 1998; Kim et al., 1995; Susic et al., 1996). Interestingly, AngII-mediated cardiac hypertrophy followed by cardiac remodeling is characterized by cardiomyocyte loss, proliferation of interstitial fibroblasts, and collagen deposition, leading to decreased contraction and increased the risk of heart failure (Anversa et al., 1996; Bishop, 1998; Colucci, 1997; Swynghedauw, 1998).

At the molecular level, AngII receptors couple to the $G_{\alpha q}$ protein-phospholipase $C$ (PLC) pathway, in which multiple second messengers such as phosphatidylinositol 4,5-bisphosphate (PIP2), inositol-1,4,5-trisphosphate (IP3), diacylglycerol and $\mathrm{Ca}^{2+}$ are included. This pathway can activate PKC and modulate membrane 
ion channels. AngII increases the delayed rectifier potassium current, $I_{k s}$ and shortens AP duration in a concentration-dependent manner. The $\mathrm{AT}_{1}$ receptor blocker valsartan and PKC inhibitors attenuate this AngII-potentiated $\mathrm{I}_{\mathrm{ks}}$, indicating that the enhanced $\mathrm{I}_{\mathrm{ks}}$ is mediated via a PKC signaling cascade (Zankov et al., 2006). Moreover, $I_{\mathrm{Ca}}$ is activated by AngII in either a PKC-dependent (Aiello and Cingolani, 2001) or -independent manner (Ichiyanagi et al., 2002).

However, AngII also has $G_{\alpha q}$ independent signaling pathways. It is well documented that AngII can mediate some of its effects via directly stimulating NOXmediated ROS production (Zhao et al., 2011). Moreover, Nishida et al. demonstrated an indirect pathway where $\mathrm{AT}_{1}$ stimulation by AngII activates $\mathrm{G}_{\alpha 12 / 13}$ proteins, which in turn cause Ras homolog gene family/Rho-associated protein kinase (Rho/ROCK)- mediated Ras-related C3 botulinum toxin substrate 1 (Rac1) activation. Rac, one of the small GTP-binding proteins, promotes the production of ROS by activating NOX (Nishida et al., 2005).

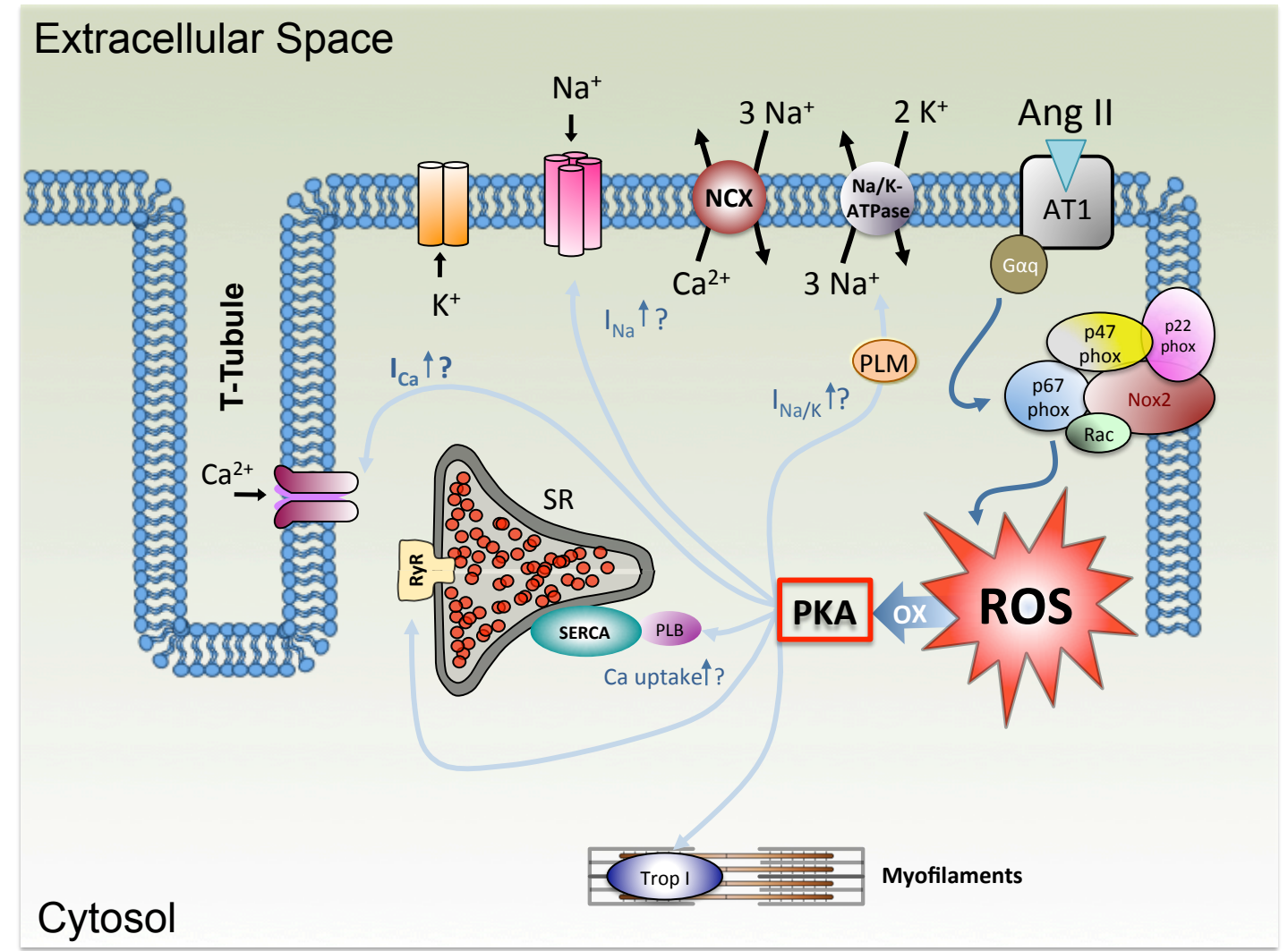

Figure 1.13. Schematic diagram showing predicted role of AngII-mediated ROS in EC coupling. Upon production, ROS can modify PKA. Therefore, may activate any of the signaling downstream to PKA. (modified from Wagner et al., 2013)

This AngII-mediated ROS can induce ventricular arrhythmias via CaMKII- 
dependent pathway, which can be blocked by CaMKII inhibitor KN-93 (Ichiyanagi et al., 2002; Zhao et al., 2011). This oxidized CaMKII can also activate p38MAPK pathway to induce apoptosis (Palomeque et al., 2009). Accordingly, mice lacking functional NOX (p47-/-mice) and mice with CaMKII inhibition (AC3I mice) have higher resistance to cell apoptosis induced by AngII (Swaminathan et al., 2011).

CaMKII signaling is also involved in AngII-mediated hypertrophy. It has been demonstrated that nuclear CaMKII activated by envelope IP3 receptor-mediated $\mathrm{Ca}^{2+}$ release can cause histone deacetylase (HDAC) phosphorylation and nuclear export. This relieves HDAC-dependent suppression of cardiomyocyte enhancer factor 2-driven transcriptions and contributes to hypertrophy (Anderson et al., 2011). Another mechanism of AngII-mediated hypertrophy could be the elevation in blood pressure after AngII exposure. Shear stress from elevated blood pressure can up-regulate AngII receptors (Ruiz-Ortega et al., 2001), which further strengthening the link between hypertension and vascular remodeling. It was found that AngII infusion for two weeks can lead to hypertension and VSMC hypertrophy in rat (Lombardi et al., 1999). Evidence consistent with a central role of AngII in the pathophysiology of heart failure also comes from the fact that the AngII receptor blockers or ACE inhibitors are clinical medications used to treat high blood pressure and heart failure (Wen et al., 2012). Interestingly, as mentioned before, PKA can also be activated by oxidation, in this thesis, the role of AngII-mediated PKA activation and downstream signaling is further investigated (fig.1.13). 


\subsection{Aim of the project}

The focus of this $\mathrm{PhD}$ thesis is to investigate the role of oxidant-dependent activation of PKA for contractile function under physiologic conditions. Moreover, the importance of redox-activated PKA for the transition from hypertrophy to heart failure is evaluated. Two different in vivo models are used: the angiotensin II-induced cardiac hypertrophy model and the chronic pressure overloadinduced hypertrophy and heart failure model.

The hypotheses to be evaluated are:

1) Redox-dependent PKA is involved in the regulation of LTCC function, which is important for excitation-contraction coupling,

2) Angiotensin-dependent regulation of $\mathrm{I}_{\mathrm{Ca}}$ includes oxidant-dependent activation of PKA type I,

3) Redox-dependent PKA is required for adaptive increases in $I_{\mathrm{Ca}}$ during pressure overload, and

4) The lack of PKA-dependent stimulation of $I_{\text {Ca }}$ upon chronic pressure overload could lead to enhanced left ventricular remodeling. 


\section{Chapter2 \\ Methods and Materiats}




\subsection{Experimental outline of this project}

Genetically modified mice and their WT littermates were enrolled in different experiments of this project to answer the hypothesis. In vitro experiments were done with isolated ventricular cardiomyocytes from these mice. Moreover, KI and WT mice were also enrolled for in vivo experiments, where they underwent transverse aortic constriction or chronic AngII infusion. To investigate contractile function and arrhythmias in vivo, echocardiography, and programmed electrical stimulation was performed two or six weeks after surgery, respectively. In parallel experiments, ventricular cardiomyocytes were isolated for further in situ measurements (fig.2.1). The following scheme summarizes the project in brief:

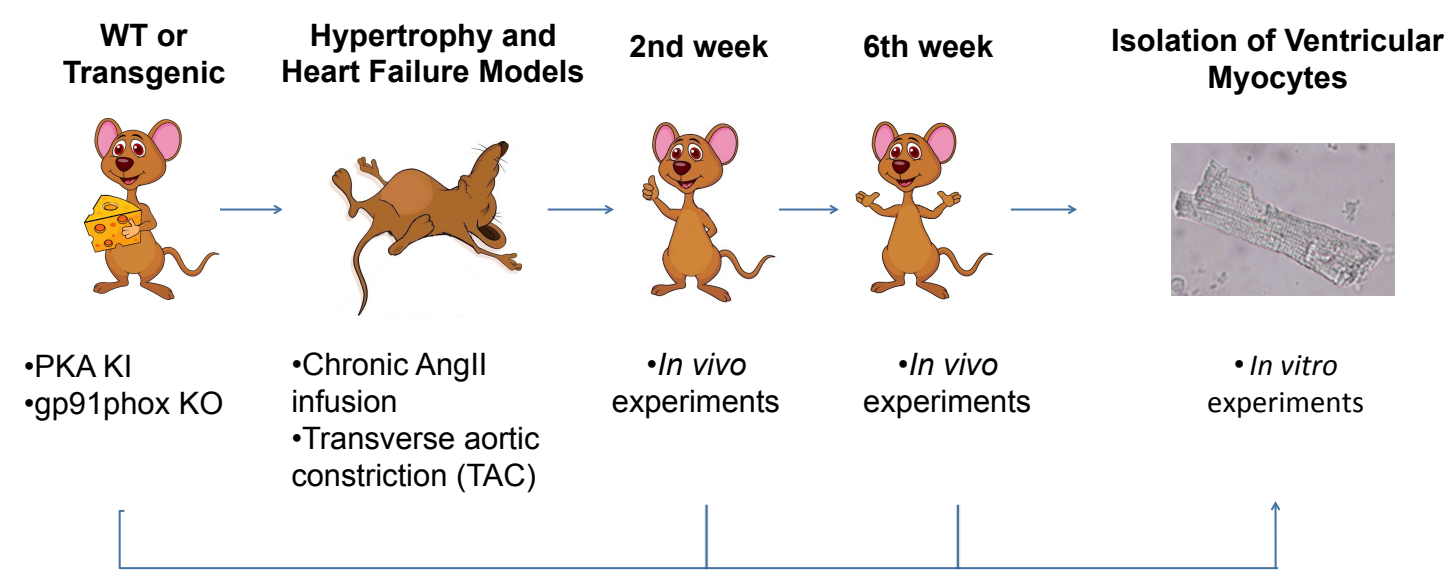

Figure 2.1. Schematic diagram showing experimental plans of the project.

\subsection{Generation of a novel 'redox dead' C17S RI knock-in (KI)} mouse

At the beginning of the project, Prkar1 gene region of the murine genome was specifically amplified by PCR. These amplified sequences were then used to introduce a substituted mutation using site-directed mutagenesis at position 17 of exon 2 to replace cysteine to serine (KI). An FRT-flanked neomycin selection marker was inserted close to the mutation, which allows selection of transfected embryonic stem cells and also favored homologous recombination with the chromosome. After screening by Southern blotting to identify if homologous recombination had occurred followed by validation of the positive clones, clones were used to transfect embryonic stem cells to generate chimera. The chimeras were directly bred with a Flp deletor for in vivo deletion of the selection marker. 
As the embryonic stem cells always go germline, chimeras were directly bred to the deletor in order to obtain germline transmission and deletion of selection marker at the same time. Pure C57BL/6 mice were used by Taconic Artemis to create this KI mouse line.

Breeding pairs were set up with heterozygous male and female mice at 6-8 weeks of age. According to Mendel's law, half of the newborn were WT and homozygous, KI and rest half were heterozygous for this genetic mutation (Het). All the male and female mice were enrolled on the experiments at 11-13 weeks of age. Gestation lasted for $\sim 3$ weeks and then the mice were weaned at 3-4 weeks of age.

\section{3. gp91phox knock out (KO) mouse line}

Mice lacking the catalytic subunit of NOX2, gp91phox, were used in this study. These mice had been generated in the lab of Prof. Ajay Shah, King's College London (Bendall et al., 2002). Because the gene is located on the X-chromosome, homozygous male mice were bred with heterozygous female mice at the age of 68 weeks. According to Mendel's law, half of the newborn were WT and homozygous or heterozygous, respectively. All the mice were enrolled in experiments at the age of $11-13$ weeks.

\subsection{Genotyping of mice}

Mouse ear and tail clippings were collected during mouse ear tagging and further used for genomic DNA isolation. DirectPCR lysis reagent Tail (Peqlab, Germany) was used to isolate DNA. $200 \mu \mathrm{l}$ of this reagent along with $2 \mu \mathrm{l}$ of proteinase $\mathrm{K}$ solution were mixed with clipped tissue. The mixture was vortexed and incubated at $55^{\circ} \mathrm{C}$ on a shaker (700 rpm) overnight. Next morning, samples were heated $45 \mathrm{~min}$ at $85^{\circ} \mathrm{C}$ to inactivate the enzymes. Samples were then cooled and quick centrifuged to sediment the hair. After collecting the supernatant, it was combined with PCR master mix to amplify the DNA using the following protocol in PCR thermocycler (table. 2.1).

Amplified DNA was separated on 1.5\% agarose gel in TBE buffer at 120V for 85 minutes. After gel electrophoresis, expected DNA bands were visualized with 
ethidium Bromide. For PKA mouse line, DNA fragments were located at $385 \mathrm{bp}$ for WT, $548 \mathrm{bp}$ for KI and both $385 \mathrm{bp}$ and $548 \mathrm{bp}$ for heterozygous genotypes (fig.2.2).

Table 2.1. Summary of PCR protocol for PKA RI mouse line.
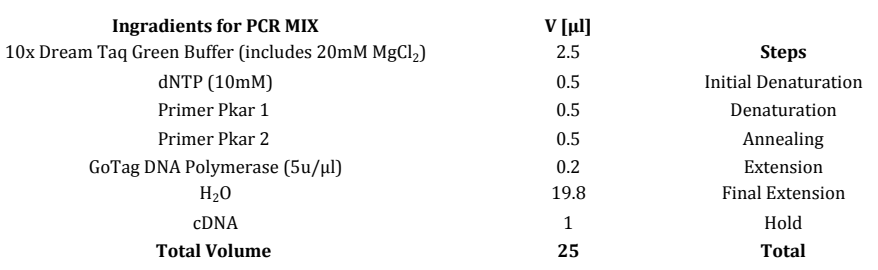

$\begin{array}{ccc}\begin{array}{c}\text { PCR protocol } \\ \text { Temparature }\left({ }^{\circ} \mathbf{C}\right)\end{array} & \text { Time } & \text { Cycles } \\ 95 & 5 \mathrm{~min} & 1 \\ 95 & 30 \mathrm{sec} & \\ 60 & 30 \mathrm{sec} & 35 \\ 72 & 2 \mathrm{~min} & \\ 72 & 10 \mathrm{~min} & 1 \\ 4 & & 1 \\ & \mathbf{2} \text { hours } & \mathbf{3 8}\end{array}$

Primers were: forward primer (Primer 1) 5'- GCTTTCCTTTACCAAGCAGG - 3' and Reverse primer (Primer 2) 5'- GTCTGTGAGTCACACTGACC -3'

$\begin{array}{llllllllllll}\text { Ladder } & \text { Het WT } & \text { Het } & \mathrm{H}_{2} \mathrm{O} & \text { WT } & \text { WT } & \text { KI } & \text { WT } & \text { KI } & \text { KI } & \text { WT }\end{array}$

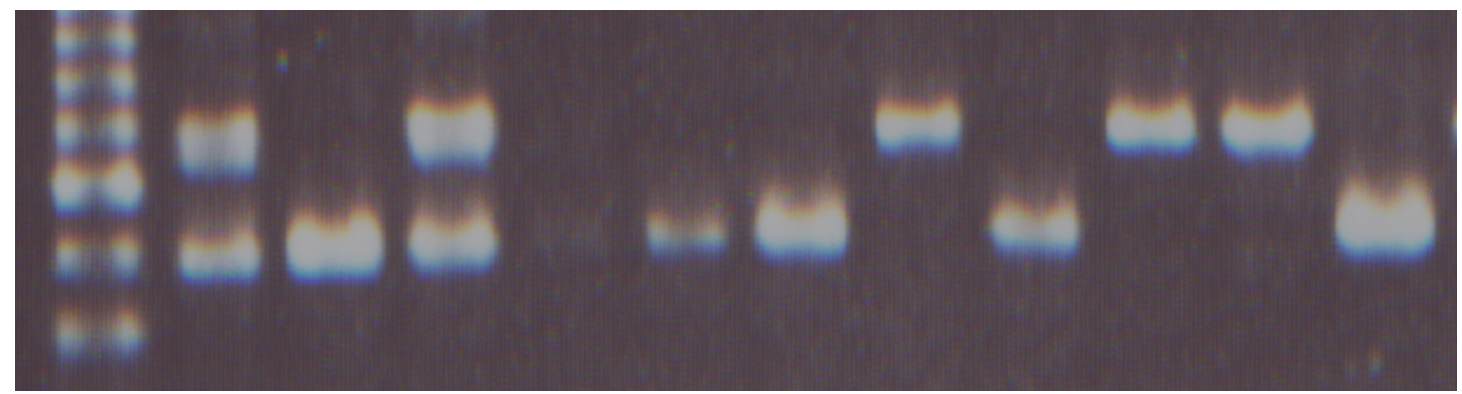

Figure 2.2. DNA gel electrophoresis revealed different mice genotypes of PKA RI mouse line.

Similar extraction protocols were used for gp91phox mice to do the PCR analysis. Afterward similar steps were used to detect the bands to distinguish between the genotypes (table.2.2).

Table 2.2. Summary of PCR protocol for gp91phox mouse line.

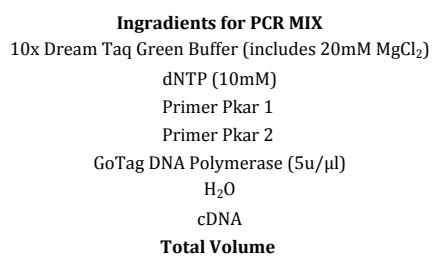

$\mathbf{V}[\boldsymbol{\mu l}]$
2.5
0.5
0.5
0.5
0.2
19.8
1
$\mathbf{2 5}$

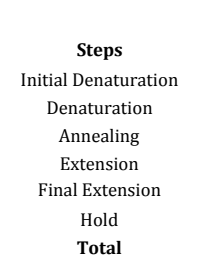

PCR protocol
Temparature $\left({ }^{\circ} \mathrm{C}\right)$
95
95
60
72
72
4

$\begin{array}{cc}\text { Time } & \text { Cycles } \\ 5 \mathrm{~min} & 1 \\ 30 \mathrm{sec} & \\ 30 \mathrm{sec} & 35 \\ 2 \mathrm{~min} & \\ 10 \mathrm{~min} & 1 \\ & 1 \\ \mathbf{2} \text { hours } & \mathbf{3 8}\end{array}$

Primers were: Primer 1:5'-AagagaAactcctctgctgtGaA- 3', Primer 2:5'-CGCTCTGGaAcCCCTGAGAAAGG-3' and Primer 3:5'-GTTCTAATTCCATCAGAAGCTTATCG-3'

Fragments were located at $240 \mathrm{bp}$ for WT, $195 \mathrm{bp}$ for KO and both bands $240 \mathrm{bp}$ and $195 \mathrm{bp}$ for heterozygous genotypes. 


\subsection{Isolation of mouse ventricular cardiomyocytes}

Langendorff-perfusion technique was used to isolate mouse ventricular cardiomyocytes. Before isolation, the perfusion apparatus was washed two times with distilled water and then with isolation buffer (table.2.3) for at least 5 minutes.

Table 2.3. Buffers and solutions used for mouse cardiomyocyte isolation. Cardiomyocyte isolation buffer, $\mathrm{pH} 7.42$ at $36.5^{\circ} \mathrm{C}$

\begin{tabular}{|c|c|}
\hline Chemicals & $\begin{array}{c}\text { Working } \\
\text { Concentration }(\mathrm{mM})\end{array}$ \\
\hline $\mathrm{NaCl}$ & 113 \\
\hline $\mathrm{KCl}$ & 4.7 \\
\hline $\mathrm{KH}_{2} \mathrm{PO}_{4}$ & 0.6 \\
\hline $\mathrm{Na}_{2} \mathrm{HPO}_{4} \times 2 \mathrm{H}_{2} \mathrm{O}$ & 0.6 \\
\hline $\mathrm{MgSO}_{4} \times 7 \mathrm{H}_{2} \mathrm{O}$ & 1.2 \\
\hline Phenol-red & 0.032 \\
\hline $\mathrm{NaHCO}_{3}$ & 12 \\
\hline $\mathrm{KHCO}_{3}$ & 10 \\
\hline HEPES & 10 \\
\hline Taurine & 30 \\
\hline Glucose & 5.5 \\
\hline BDM & 10 \\
\hline $\mathrm{dd}_{2} \mathrm{O}$ & to $1000 \mathrm{ml}$ \\
\hline \multicolumn{2}{|l|}{ Digestion buffer } \\
\hline 1x Tyrode & $20 \mathrm{ml}$ \\
\hline Liberase TM & $1.5 \mathrm{mg}$ \\
\hline Trypsin 10x, 2.5\% & $111.2 \mu \mathrm{l}$ \\
\hline $10 \mathrm{mM} \mathrm{CaCl}_{2}$ & $25 \mu \mathrm{l}$ \\
\hline Stop solution 1 & volume $(\mu \mathrm{l})$ \\
\hline Isolation buffer & 2250 \\
\hline Bovine calf serum (BCS) & 250 \\
\hline $10 \mathrm{mM} \mathrm{CaCl}_{2}$ & 3.125 \\
\hline Stop solution 2 & volume (ml) \\
\hline Isolation buffer & 23.75 \\
\hline Bovine calf serum (BCS) & 1.25 \\
\hline Total volume & 25 \\
\hline \multicolumn{2}{|l|}{ Calcium building solutions } \\
\hline 1. Solution $2(\mathrm{ml})$ & 5 \\
\hline $100 \mathrm{mM} \mathrm{CaCl}_{2}(\mu \mathrm{l})$ & 5 \\
\hline Final concentration [mmol/l] & 0.1 \\
\hline 2. Solution $2(\mathrm{ml})$ & 5 \\
\hline $100 \mathrm{mM} \mathrm{CaCl}_{2}(\mu \mathrm{l})$ & 10 \\
\hline Final concentration [mmol/l] & 0.2 \\
\hline 3. Solution $2(\mathrm{ml})$ & 5 \\
\hline $100 \mathrm{mM} \mathrm{CaCl}_{2}(\mu \mathrm{l})$ & 20 \\
\hline Final concentration $[\mathrm{mmol} / \mathrm{l}]$ & 0.4 \\
\hline 4. Solution $2(\mathrm{ml})$ & 10 \\
\hline $100 \mathrm{mM} \mathrm{CaCl}_{2}(\mu \mathrm{l})$ & 80 \\
\hline Final concentration $[\mathrm{mmol} / \mathrm{l}]$ & 0.8 \\
\hline
\end{tabular}

Mice were anesthetized in a gas chamber with isoflurane 2-4\% (Abbott, USA). After cervical dissection, the abdomen was opened, and the heart was carefully located by cutting the diaphragm from the abdominal side. The heart was removed by gently cutting all vessels at the heart base and then washed with isolation buffer. The ascending aorta of the heart was immediately cannulated to avoid blood clotting in small capillaries. Isolation buffer was directly perfused 
with a flow rate of $3 \mathrm{ml} / \mathrm{min}$ for 3 minutes at $37^{\circ} \mathrm{C}$. After that, perfusion with digestion buffer was continued for another 4-5 $\mathrm{min}$ at the same flow rate. Digestion buffer contained liberase and trypsin to facilitate tissue dissociation and cell harvesting.

After finishing the digestion, the heart was cut from the cannula and placed in a petri dish containing $5.0 \mathrm{ml}$ of digestion buffer including BCA, which stops all the enzymatic reactions. The heart was then cut into small pieces and pipetted several times gently to detach cardiomyocytes from each other. Cells were then passed through a nylon mesh to remove tissue debris. This was followed by stepwise (5 steps) increase of the external $\mathrm{Ca}^{2+}$ concentration by exposing the cells to solutions with increasing $\mathrm{Ca}^{2+}$ concentration (table.2.3). The solution was changed in every $7 \mathrm{~min}$ until $\mathrm{Ca}^{2+}$ reached to $0.8 \mathrm{mmol} / \mathrm{L}$ concentration. For each step, only sedimented live and viable cells were taken for the following step, other cells in the supernatant were carefully discarded.

\subsection{Whole-heart perfusion experiments}

In separate experiments, the Langendorff-setup was used to perfuse saline solutions containing AngII ( $1 \mu \mathrm{mol} / \mathrm{L})$ to mouse heart for $10 \mathrm{~min}$. For control experiments, only saline solution was used during perfusion. After that, the heart was removed, and ventricular tissue was quickly frozen for further protein analysis.

\subsection{Mitochondrial and cytosolic ROS detection}

Mitochondrial "leaky" electrons from electron transport chain interact with molecular oxygen to form superoxide anion, which is the predominant reactive oxygen species found in mitochondria. MitoSOX Red (from life technologies), a cationic derivative of dihydroethidum, was used in this project to selectively detect these superoxide molecules in actively respiring mitochondria (fig.2.4). Oxidation of this dye by superoxide results in 2-hydroxyethidium, which exhibited a fluorescence excitation peak at $\sim 400 \mathrm{~nm}$ and emission at around $590 \mathrm{~nm}$. Reactive oxygen species other than superoxide cannot form an oxidized product of ethidium with an excitation spectrum around 400nm. This makes MitoSOX Red precise to detect the change in mitochondrial superoxide production (Robinson et al., 2008). 


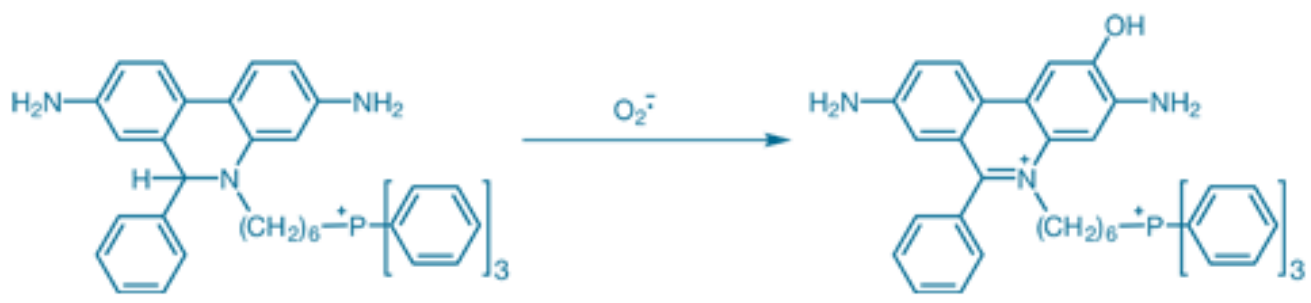

Figure 2.4. Oxidation of MitoSox Red mitochondrial superoxide indicator to 2-hydroxy-5(triphenylphosphonium) hexylethidium by superoxide $\left(\mathrm{O}_{2}{ }^{{ }^{-}}\right)$. (taken from thermofisher.com)

In contrast, cell-permeable CellROX orange (from life technologies) was used to detect cytosolic oxygen and nitrogen derived oxidants. ROS. In its reduced state, the dye is nonfluorescent or very weakly fluorescent; however, upon oxidation, it becomes brightly fluorescent, with excitation/emission maxima at 545/565 nm.

To quantify the ROS, freshly isolated ventricular cardiomyocytes were plated on laminin-coated glass chambers for at least 20 minutes. MitoSOX stock solution (5 mmol/L in DMSO) were prepared and kept in the freezer. This stock solution was used to make $5 \mu \mathrm{M}$ MitoSOX working solution with $\mathrm{Ca}^{2+}$-free Tyrode immediately before the experiment (table.2.4). Isolation buffer was carefully removed from the cell containing chambers, to add this working MitoSOX solution for next 20-30 more minutes. This allows the dye to enter the cell and interact with ROS. After incubation, the chamber was gently washed three times with Tyrode. Chambers were then mounted on a confocal microscope (Zeiss LSM 5 Pascal, 40x oil immersion objective, NA 1.3). MitoSOX was excited by laser light at $488 \mathrm{~nm}$ in confocal mode (pin hole size 1 airy unit). Emitted fluorescence was detected by a photomultiplier using a $560 \mathrm{~nm}$ long pass emission filter. Averages of four frames were used to record an image and scanning was further followed for continuous 15 minutes (1 image in every $\mathrm{min}$ ) for each cell. Increase in fluorescence intensity was analyzed using specified regions of interest (ROI) after exposure to either AngII $1 \mu \mathrm{mol} / \mathrm{L}$ or vehicle.

Cytosolic ROS was detected using CellROX orange dye, which can bind to cytosolic ROS but not to the mitochondrial or nuclear ROS. The CellROX dye was bought as a ready-to-use stock solution containing $2.5 \mathrm{mmol} / \mathrm{L}$ CellROX in DMSO. This stock was then diluted to a working concentration of $2.5 \mu \mathrm{M}$ in $\mathrm{Ca}^{2+}$-free 
Tyrode. Similar to MitoSOX, cells were loaded with the dye for 20-30 min in the dark. CellROX fluorescence was exited using a $555 \mathrm{~nm}$ laser on the stage of a confocal microscope (LSM Pascal 5). Emitted fluorescence was detected with a photomultiplier (560 nm long pass filter) as stated above.

Table 2.4. The composition of solution used for fluorescence measurements.

$\begin{array}{lc}\begin{array}{l}\text { Tyrode (Solution for fluorescence measurement), } \mathbf{p H} \\ \text { Corking Concentration } \\ \text { Chemicals }\end{array} \\ \text { (mM) } \\ \mathrm{NaCl} & 140 \\ \mathrm{KCl} & 5.4 \\ \mathrm{MgCl}_{2} & 1 \\ \mathrm{HEPES} & 5 \\ \mathrm{Glucose} & 10 \\ \mathrm{CaCl}_{2} & 1 \\ \mathrm{ddH}_{2} \mathrm{O} & \text { to } 1000 \mathrm{ml} \\ \text { Caffeine (if needed) } & 10\end{array}$

\subsection{Measurement of subcellular distribution of PKA RI using immunocytochemistry}

Mouse ventricular cardiomyocytes were plated, immediately after the isolation, on chamber slides ( 4 well glass slide, Nunc, USA), coated with laminin to ensure cellular attachment to the bottom of the slides. After $20 \mathrm{~min}$, the cardiomyocytes were stimulated for 10-15 min by external exposure to AngII (1 $\mu \mathrm{M}$, in Tyrode, table. 4). After that, cells were fixated to the slides by exposing them to $99 \%$ icecold ethanol for $20-30$ minutes at $-20^{\circ} \mathrm{C}$. After fixation, the cells were washed with 1X PBS (three times for five minutes) and blocked with 5\% BSA-PBST blocking solution either at room temperature for one hour in a shaker or overnight at $4^{\circ} \mathrm{C}$ to minimize nonspecific staining. After blocking, cardiomyocytes were washed with $1 \mathrm{X}$ PBS (three times five minutes each) and cells were subsequently incubated with primary antibody (monoclonal mouse anti-PKA RI, BD Transduction Laboratories, 1:100) diluted with antibody diluent (Dako) overnight at $4^{\circ} \mathrm{C}$. After washing with $1 \mathrm{X}$ PBS (six times five minutes), the cells were incubated with the fluorescent-labeled secondary antibody (goat anti-mouse Alexa 488 or goat anti-rabbit Alexa 555, 1:200, Invitrogen) diluted in antibody dilution buffer for 2 hours at room temperature in the dark.

Cells were then washed as before to remove unbound secondary antibodies and covered with VECTASHIELD HardSet mounting medium (Vector Laboratories) 
for further analysis. Fluorescence was excited on the stage of a confocal microscope (Zeiss Pascal 5) using laser light at 488 or $555 \mathrm{~nm}$ respectively. Emitted fluorescence was detected by a photomultiplier (emission filter: $560 \mathrm{~nm}$ long pass filter). For all experiments, pinhole size was set at 1 airy unit. Cells were frame scanned, and fluorescence intensity was analyzed in different regions of interest (ROI). For control experiments, no primary antibody was used.

\subsection{Measurement of intracellular calcium in isolated ventricu- lar cardiomyocytes}

Each cycle of contraction and relaxation is regulated by the transient rise and decrease of cytosolic $\mathrm{Ca}^{2+}$. These $\mathrm{Ca}^{2+}$ transients are underlying the myofilament interaction that leads to contraction. Inability to maintain proper $\mathrm{Ca}^{2+}$ transients can lead to severe impairment of contractile function on the organ level. To measure the $\mathrm{Ca}^{2+}$ transients, the fluorescent dye Fura-2, an aminopolycarboxylic acid, was used. Membrane permeable ester form of Fura-2, named Fura-2acetoxymethyl ester (Fura-2 AM), freely diffuses across the cell membrane, where esterases de-esterify the Fura- 2 molecules. With time, cells accumulate Fura-2 molecules, as the salt form cannot leave the cell, whereas the ester form still diffuses into the cytoplasm. Being a polar salt, Fura-2 can bind to free intracellular $\mathrm{Ca}^{2+}$, which ultimately changes its fluorescence properties. The $\mathrm{Ca}^{2+}$ bound Fura-2 has an excitation maximum at $340 \mathrm{~nm}$, whereas the free Fura-2 salt has its maximum at $380 \mathrm{~nm}$. The measurement of Fura-2 fluorescence emission at both excitation wavelengths allows rendering the signal independent from variations in dye concentration or cell thickness, The ratio of fluorescence emission at 340 and $380 \mathrm{~nm}$ excitation was used in the present study as a measure of intracellular $\mathrm{Ca}^{2+}$.

At first, $50 \mu \mathrm{g}$ of Fura- 2 AM dye was mixed with $44 \mu \mathrm{l}$ of DMSO to produce a stock concentration of $10 \mathrm{mmol} / \mathrm{L}$. This stock was then used to prepare working concentrations of $10 \mu \mathrm{mol} / \mathrm{L}$ by dilution using $\mathrm{Ca}^{2+}$-free Tyrode solution in the presence of $0.02 \%$ (w/v) pluronic acid (Molecular Probes, Eugene) (table.2.6).

Freshly isolated cardiomyocytes were plated on laminin-coated IonOptix glass 
chambers for 15 minutes, which allowed the cells to attach to the bottom of the chamber. The isolation buffer was removed and replaced with Fura-2 acetoxymethyl ester (AM) at a working concentration of $10 \mu \mathrm{mol} / \mathrm{L}$ for 20 minutes in the dark at room temperature. After dye loading, cells were mounted onto the stage of an inverted microscope (Motic AE31 inverted microscope, objective 40X, NA 1.4; Olympus).

The chamber was washed for 10 minutes using the perfusion system with normal $1 X$ Tyrode solution (table.2.5) with a flow rate of $80 \mathrm{ml} / \mathrm{h}$ at $37^{\circ} \mathrm{C}$. This allows de-esterification of the dye as well removal of the excess dye from the chamber. Temperature of the solution was maintained by an in-line solution heater (SF-28, Harvard Apparatus, USA). Bright field images of the cardiomyocytes were monitored using a MyoCam (voltage 20\% above the threshold) at 1 $\mathrm{Hz}$ to induce cell twitching.

Table 2.5. The composition of solution used for epi-fluorescence measurements.

\begin{tabular}{|c|c|}
\hline Chemicals & $\begin{array}{l}\text { Working Concentration } \\
\qquad(\mathrm{mM})\end{array}$ \\
\hline $\mathrm{NaCl}$ & 140 \\
\hline $\mathrm{KCl}$ & 4 \\
\hline $\mathrm{MgCl}_{2}$ & 1 \\
\hline HEPES & 5 \\
\hline Glucose & 10 \\
\hline $\mathrm{CaCl}_{2}$ & 1 \\
\hline $\mathrm{ddH}_{2} \mathrm{O}$ & to $1000 \mathrm{ml}$ \\
\hline Caffeine (if needed) & 10 \\
\hline
\end{tabular}

At steady-state condition, stimulation frequency was increased stepwise from 0.5 to $4 \mathrm{~Hz}$ to evaluate frequency-dependent cell shortening. During this time, Fura-2 was alternatively excited with light at wavelengths of 340 and $380 \mathrm{~nm}$ using a Hyperswitch (Ionoptix). The latter consists of a rotating mirror (250 Hz) that directs the ultra-violet light generated by the $75 \mathrm{~W}$ xenon arc lamp (Ushio, Japan) to excitation filters of $340( \pm 15 \mathrm{~nm})$ or $380 \mathrm{~nm}( \pm 15 \mathrm{~nm})$, respectively. The emitted fluorescence was detected by a photomultiplier (emission filter at 510 nm; IonOptix Corp, Milton, Mass) at a sampling frequency of $250 \mathrm{~Hz}$. The obtained fluorescence intensity values were background-subtracted, and the ratio of emitted fluorescence at excitation light of $340 \mathrm{~nm}$ and $380 \mathrm{~nm}\left(\mathrm{~F}_{340} / \mathrm{F}_{380}\right)$ was calculated.

$\mathrm{F}_{340} / \mathrm{F}_{380}$ correlated with cytosolic $\mathrm{Ca}^{2+}$ and was used to measure $\mathrm{Ca}^{2+}$ transient 
amplitude and diastolic $\mathrm{Ca}^{2+}$. Also, the decay of the $\mathrm{Ca}^{2+}$ transient was fitted to a single exponential. Its time constant, tau, was used as a measure of $\mathrm{Ca}^{2+}$ transient decay kinetic. For some experiments, rapid external application of $10 \mathrm{mM}$ caffeine resulted in the complete release of SR $\mathrm{Ca}^{2+}$. The resulting caffeine-transient was used as a measure of SR $\mathrm{Ca}^{2+}$ content.

\subsection{Measurement of calcium sparks using confocal microscope}

Calcium sparks are spontaneous SR calcium release events. Characterization of sparks has provided mechanistic insights into the development of a variety of cardiac diseases. Fluo-4 AM, a fluorine-substituted cell permeable acetoxymethyl ester analog of Fluo-3, is used to detect $\mathrm{Ca}^{2+}$ sparks in intact cardiomyocytes. Free unbound form of Fluo-4 is non-fluorescent, however, upon binding to $\mathrm{Ca}^{2+}$, excitation at $488 \mathrm{~nm}$ generates a strong fluorescence emission at $516 \mathrm{~nm}$. Moreover, the change in fluorescence emission is greatest for $\mathrm{Ca}^{2+}$ concentrations in the range of 10-1000 $\mathrm{nM}$, which renders the dye ideal for detection of cytosolic $\mathrm{Ca}^{2+}$ in cardiomyocytes.

Fresh mouse cardiomyocytes were plated on laminin-coated chambers for 15 minutes to allow the cells to attach to the bottom of the chambers. After that, isolation buffer was removed and replaced with $10 \mu \mathrm{M}$ of Fluo-4-AM dye dissolved in $\mathrm{Ca}^{2+}$-free Tyrode in the dark for 15 minutes. The acetoxymethyl ester dye (AM) entered to the cell and was hydrolyzed in the cytosol to free salt, which can bind $\mathrm{Ca}^{2+}$ ions. Following dye loading, the buffer was exchanged for normal Tyrode solution containing $1 \mathrm{mM} \mathrm{Ca}^{2+}$ at room temperature. This resulted in washing out of excess Fluo-4-AM; however, only the polar Fluo-4 salt remains in the cytoplasm.

$\mathrm{Ca}^{2+}$ sparks were recorded using a laser scanning confocal microscope (LSM 5 Pascal, Zeiss) with a $40 \times$ oil-immersion objective. Fluo-4 AM was excited via an argon laser at $488 \mathrm{~nm}$ and emitted fluorescence was collected through a $515 \mathrm{~nm}$ long-pass emission filter. Fluorescence images were recorded in the line-scan mode with 512 pixels per line, pixel time $0.64 \mu$ s (1040 lines per sec), pixel size $0.07 \mu \mathrm{m} \times 0.07 \mu \mathrm{m}$, at 1 airy unit. Cells were electrical field-stimulated at $0.5 \mathrm{~Hz}$ and scans were acquired immediately after stopping the electrical field stimula- 
tion. After subtraction of background fluorescence, Fluo-4 fluorescence intensity was normalized to diastolic fluorescence intensity $\left(\mathrm{F}_{0}\right)$ as $\mathrm{F} / \mathrm{F}_{0}$. Line scan images were imported into Image J software, and $\mathrm{Ca}^{2+}$ sparks were automatically detected by mathematical algorithms using the Sparkmaster plugin (Picht et al., 2007). After manual verification, $\mathrm{Ca}^{2+}$ sparks were analyzed according to frequency (normalized to cell volume and scan rate as sparks $\mathrm{x} \mathrm{pl}^{-1} \mathrm{x} \mathrm{s}^{-1}$ ), $\mathrm{Ca}^{2+}$ spark amplitude in $\Delta \mathrm{F} / \mathrm{F}_{0}, \mathrm{Ca}^{2+}$ spark width at half maximum (full width half maximum FWHM, $\mu \mathrm{m}$ ), and $\mathrm{Ca}^{2+}$ spark duration at half maximum (full duration half maximum, FDHM, ms).

\subsection{Evaluation of calcium channel function using patch-clamp experiments}

Whole-cell patch clamp technique was used to evaluate voltage-gated $\mathrm{Ca}^{2+}$ channel activity in real-time by measuring macroscopic $\mathrm{Ca}^{2+}$ current. In this method, current can be recorded through multiple channels simultaneously, over the entire cell membrane. This method has more precision than any other cell biology techniques with temporal resolution $<1 \mathrm{~ms}$, which makes it unique to probe the molecular physiology, pharmacology, and biophysics of this channel protein.

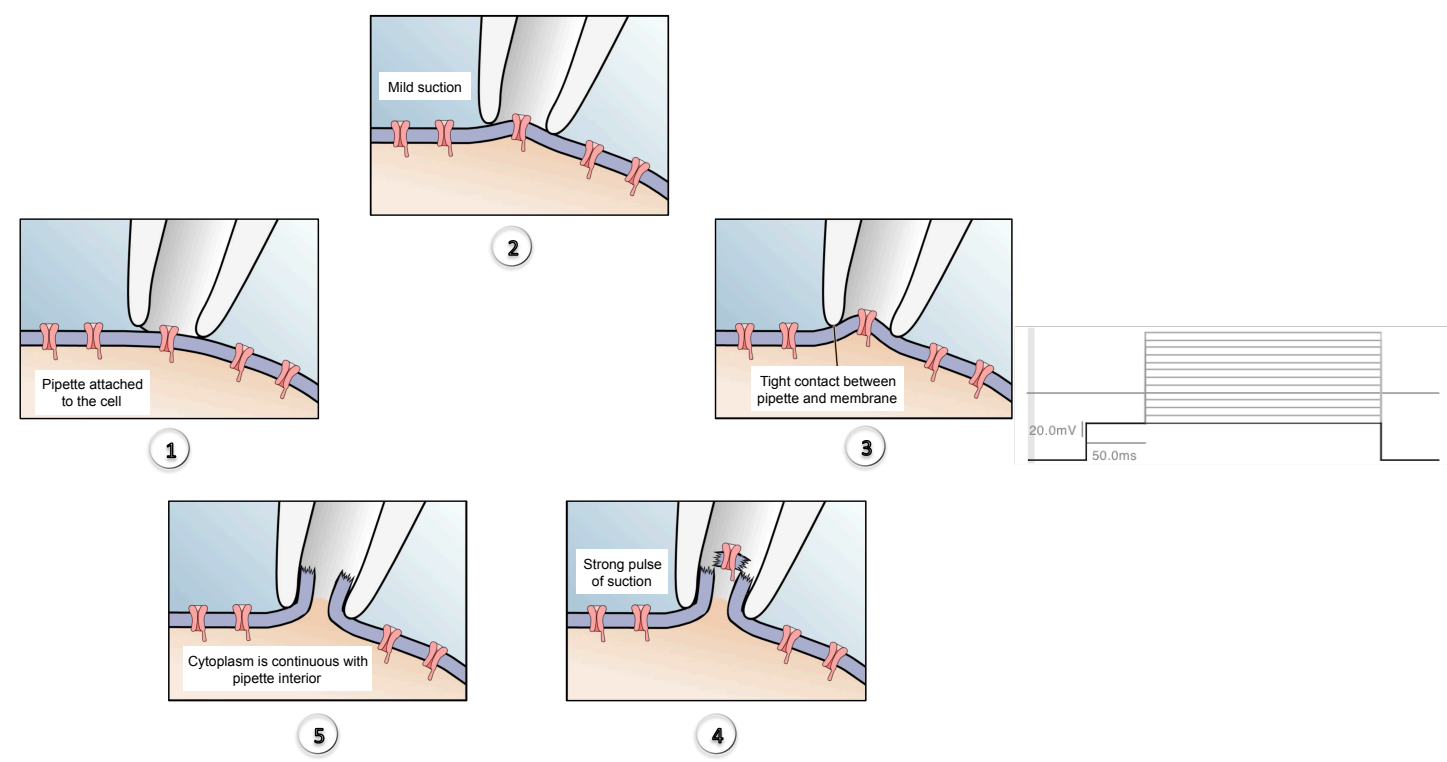

Figure 2.5. Schematic diagram showing the procedure and protocol for whole cell $I_{C a}$ measurement. In the left panel, diagrams show the principle of whole cell patch technique, where a sharp pipette is used to attach to the cell membrane $(1,2,3)$. By applying a rapid suction with this pipette, the cell is carefully ruptured (4), so that all the cytoplasmic contents can get mixed with pipette solution (5). Thus, allowing the measurement of $\mathrm{Ca}^{2+}$ currents at single cell level. Right panel shows the voltage-step protocol used for $\mathrm{Ca}^{2+}$ current recordings. 
ly good quality recordings with minimal or no leak currents were used for further analysis.

Current-voltage (I-V) relationship was determined by increasing the voltage stepwise from $-40 \mathrm{mV}$ to $+80 \mathrm{mV}$ in $10 \mathrm{mV}$ steps from a holding potential of -90 $\mathrm{mV}$ (fig.2.6). Each measurement pulse (duration 200ms) was preceded by a $50 \mathrm{~ms}$ pre-pulse to $-40 \mathrm{mV}$ to inactivate the voltage-gated sodium currents. All currents were acquired at room temperature and normalized to membrane capacitance, $\mathrm{C}_{\mathrm{m}}$.

\subsection{Protein analysis of cardiomyocyte lysates and heart ho- mogenates using western blot technique}

Western blot technique was used to determine the expression and posttranslational modifications of $\mathrm{Ca}^{2+}$ regulatory proteins. Two different types of samples were used for this technique: isolated cardiomyocytes or whole mice hearts. In addition, human atrial appendage biopsies obtained from patients, who had undergone aortocoronary bypass grafting, was also used. These patients suffered from atrial fibrillation (AF) and obstructive sleep apnea (OSA). For AF patients, mild (paroxysmal) and severe (permanent) AF were analyzed separately. Paroxysmal AF means recurrent episodes that stop on their own in less than 7 days; however, permanent AF means ongoing long-term episodes. For the OSA patients, the severity of the sleep disorder is quantified by measuring the apnea-hypopnea index (AHI), which is defined as number of events reported per hour.

Isolated cardiomyocytes were exposed to $100 \mu \mathrm{M} \mathrm{H}_{2} \mathrm{O}_{2}$ for 10 minutes, to evaluate the effect of $\mathrm{H}_{2} \mathrm{O}_{2}$ on $\mathrm{Ca}^{2+}$ handling proteins in cardiomyocytes. After that, cells were centrifuged, and pellets were lysed using lysis buffer (table.2.7). An equal volume of sample buffer containing either $\beta$-mercaptoethanol or maleimide was directly added to the samples. Samples were then heated at $55^{\circ} \mathrm{C}$ to heat denatured target proteins as well as for maximal maleimide binding. SDSPAGE was performed afterward to detect the target proteins (table. $2.8 \& 2.9$ ). 
Table 2.7. The compositions of western blot lysis and sample buffers.

Lysis buffer

Chemicals

Tris- $\mathrm{HCl}, \mathrm{pH} 7.4$

$\mathrm{NaF}$

$\mathrm{Na}_{3} \mathrm{VO}_{4}$

Leupeptin

Pepstatin A

Aprotinin

Benzamidine

PMSF

EDTA, pH 7.2

$\mathrm{MnCl}_{2}$

Calpain1 inhibitor

Calpain2 inhibitor

$\mathrm{ddH}_{2} \mathrm{O}$

Sample buffer, $2 \mathrm{x}$

Chemicals
Tris- $\mathrm{HCl}, \mathrm{pH} 6.8$
SDS
Glycerol
Bromphenol Blue
$\beta$-mercaptoethanol/Maleimide
$\mathrm{dd}_{2} \mathrm{O}$

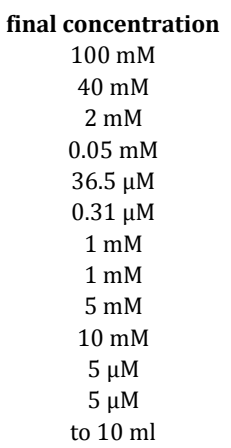

final concentration

$100 \mathrm{mM}$

$4 \%$

$20 \%$

$2 \mathrm{mg} / \mathrm{ml}$

$10 \% / 100 \mathrm{mM}$

to $20 \mathrm{ml}$

Maleimide was used to block the free cysteine so that they cannot form disulfide bond during the subsequent steps of western blot. This allows detecting the amount of oxidized as well as reduced proteins in the sample, which is in contrast to conventional western blot technique, where $\beta$-mercaptoethanol is used to deliberately reduce all the proteins in the sample. Thus, maleimide-treated samples give an opportunity to assess oxidative stress-induced protein modifications in the cell with just small modification in this sample preparation, without changing any other western blotting steps. For example, type I regulatory subunit of PKA can form intermolecular dimer upon oxidation, which can be visualized in this method. Thus, oxidized type I PKA showed a band around 100kD and reduced one at $50 \mathrm{kDa}$.

In the case of frozen heart samples, tissue was pulverized and suspended in sample lysis buffer in $10 \% \mathrm{w} / \mathrm{v}$ ratio. Lysed samples were immediately mixed with $2 x$ sample buffer containing either $\beta$-mercaptoethanol or maleimide; respectively for reducing and non-reducing western blot analysis. Samples were then heated for $10 \mathrm{~min}$ at $55^{\circ} \mathrm{C}$ and kept in the freezer for further electrophoresis and blotting steps. These prepared samples were then run on SDSpolyacrylamide gel at $100 \mathrm{~V}$ to electrophoretically separate proteins on the basis of their molecular weight. Gels were prepared in house using the following composition (table.2.8) or bought ready to use ones from Bio-Rad. 
Table 2.8. SDS-PAGE resolving and stacking gel compositions.

\begin{tabular}{|c|c|c|c|c|c|c|c|c|}
\hline \multirow{3}{*}{ Components } & \multicolumn{4}{|c|}{ Resolving gel } & & & \multicolumn{2}{|c|}{ Stacking gel } \\
\hline & \multicolumn{2}{|c|}{$7.5 \%$ gel } & \multicolumn{2}{|c|}{$10 \%$ gel } & \multicolumn{2}{|c|}{$15 \%$ gel } & \multirow{2}{*}{ Component } & \multirow{2}{*}{$\begin{array}{c}5 \% \text { gel } \\
5 \mathrm{ml}\end{array}$} \\
\hline & $10 \mathrm{ml}$ & $20 \mathrm{ml}$ & $10 \mathrm{ml}$ & $20 \mathrm{ml}$ & $10 \mathrm{ml}$ & $20 \mathrm{ml}$ & & \\
\hline Rotiphorese Gel $30(\mathrm{ml})$ & 2.5 & 5 & 3.33 & 6.66 & 4.45 & 8.9 & Rotiphorese Gel $30(\mathrm{ml})$ & 0.83 \\
\hline $4 \times$ Tris/SDS, pH8.8 (ml) & 2.5 & 5 & 2.5 & 5 & 2.5 & 5 & $4 \times$ Tris/SDS, pH $6.8(\mathrm{ml})$ & 1.25 \\
\hline $\mathrm{ddH}_{2} \mathrm{O}(\mathrm{ml})$ & 4.9 & 9.8 & 4.1 & 8.2 & 2.45 & 4.9 & $\mathrm{ddH}_{2} \mathrm{O}(\mathrm{ml})$ & 2.86 \\
\hline $10 \%$ APS $(\mu \mathrm{l})$ & 100 & 200 & 100 & 200 & 100 & 200 & $10 \%$ APS $(\mu \mathrm{l})$ & 50 \\
\hline TEMED $(\mu \mathrm{l})$ & 10 & 20 & 10 & 20 & 10 & 20 & TEMED $(\mu \mathrm{l})$ & 5 \\
\hline
\end{tabular}

After electrophoresis, proteins were transferred to nitrocellulose and/or PVDF membrane by semi-dry blotting technique. Once transferred, membranes were blocked with $5 \%$ bovine serum albumin or with $3 \%$ normal goat serum for 1-2 hours at room temperature. Desired primary antibody was diluted in same diluent as used for blocking and incubated overnight at $+4^{\circ} \mathrm{C}$ with gentle shaking.

Table 2.9. The composition of different buffers used in western blot.

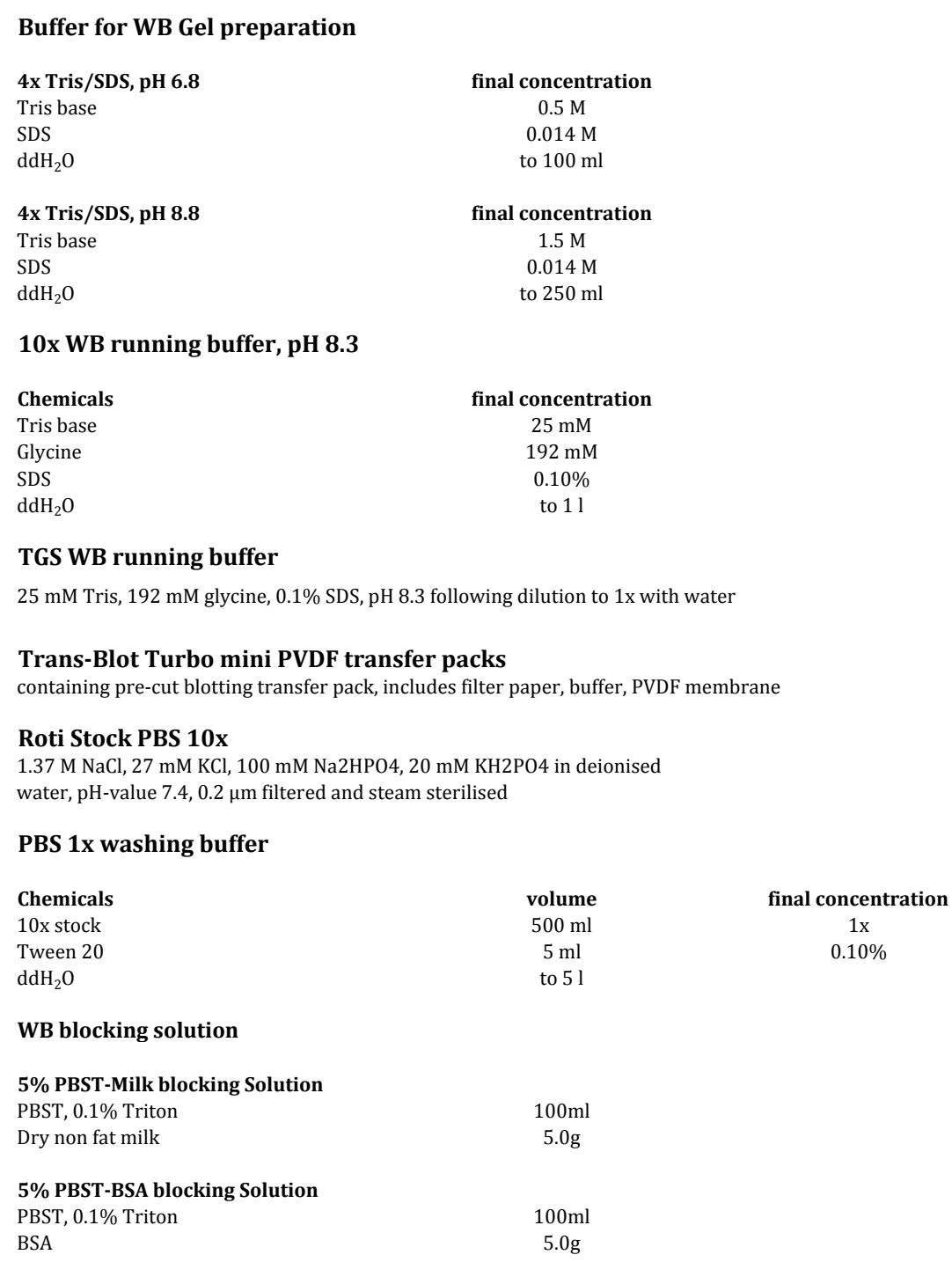

Next day, membranes were washed and incubated with horseradish peroxidaseconjugated secondary antibodies for 1-2 hours (table.2.10). Following washing, 
membranes were exposed to peroxidase substrates to detect the signals. Bands were analyzed by densitometry and normalized to housekeeping proteins.

Table 2.10. List of antibodies used in this study.

$\begin{array}{ll}\begin{array}{l}\text { Primary antibody } \\ \text { Antibody name }\end{array} & \text { Host } \\ & \\ \text { PKA RI, 610165 } & \text { Mouse } \\ \text { PKA C, 610980 } & \text { Mouse } \\ \text { PKC } \alpha \text {, ab32376 } & \text { Rabbit } \\ \text { Phospholanban, 05-205 } & \text { Mouse } \\ \text { p-Phospholamban Ser 16,A010-12 AP } & \text { Rabbit } \\ \text { CaMKII, PA5-22168 } & \text { Rabbit } \\ \text { p-CaMKII Thr 287, MA1-047 } & \text { Mouse } \\ \text { LTCC, AB5156 } & \text { Rabbit } \\ \text { RyR2, HPA020028 } & \text { Rabbit } \\ \text { p-RyR Ser 2808, A010-30 AP } & \text { Rabbit } \\ \text { SERCA 2a, MA3-919 } & \text { Mouse } \\ \text { GAPDH, BTMC-A473-9 } & \text { Mouse } \\ \text { a/b-Tubulin, 2148 } & \text { Rabbit } \\ & \\ \text { p-LTCC Ser 1928, A010-70 } & \text { Rabbit } \\ \text { Secondary antibody } & \\ \text { Peroxide conjugated Goat Anti Mouse Ab, 115-035-062 } & \\ \text { Peroxide conjugated Horse Anti Mouse Ab, 7076 } & \\ & \\ \text { Peroxide conjugated Goat Anti Rabbit Ab, 111-035-144 } & \\ \text { Peroxide conjugated Goat Anti Rabbit Ab, 7074 } & \end{array}$

\begin{tabular}{ccc}
\multicolumn{3}{c}{ Dilution } \\
\\
$1^{\circ} \mathrm{Ab}$ in $5 \%$ BSA PBST blocking solution & $2^{\circ} \mathrm{Ab}, 1 \%$ BSA PBST blocking solution & Company \\
3000 & 20000 & BD Bioscience \\
3000 & 20000 & BD Bioscience \\
3000 & 20000 & Abcam \\
15000 & 20000 & Merck Millipore \\
5000 & 10000 & Badrilla \\
10000 & 10000 & Invitrogen \\
4000 & 10000 & Invitrogen \\
5000 & 20000 & Merck Millipore \\
15000 & 30000 & Sigma-Aldrich \\
5000 & 10000 & Badrilla \\
20000 & 30000 & Invitrogen \\
30000 & 30000 & Biotrend \\
2500 & 20000 & Cell Signaling \\
& & \\
$3 \%$ Goat serum blocking solution & $3 \%$ Goat serum blocking solution & \\
10000 & 20000 & Badrilla \\
& & \\
& as mentioned above & Jackson Laboratory \\
& 5000 & Cell Signaling \\
& & Jackson Laboratory \\
& as mentioned above & Cell Signaling
\end{tabular}

\subsection{In-vitro cyclic adenosine monophosphate assay}

Cyclic adenosine monophosphate (cAMP) was analyzed with a competitive colorimetric immunoassay for quantitative determination of intracellular cAMP in tissues according to manufacturer protocol (fig.2.6, Enzo). Briefly, frozen heart tissue was ground in liquid nitrogen and homogenized in 0.1M HCL, which stabilizes cAMP and inhibits endogenous phosphodiesterase as well.

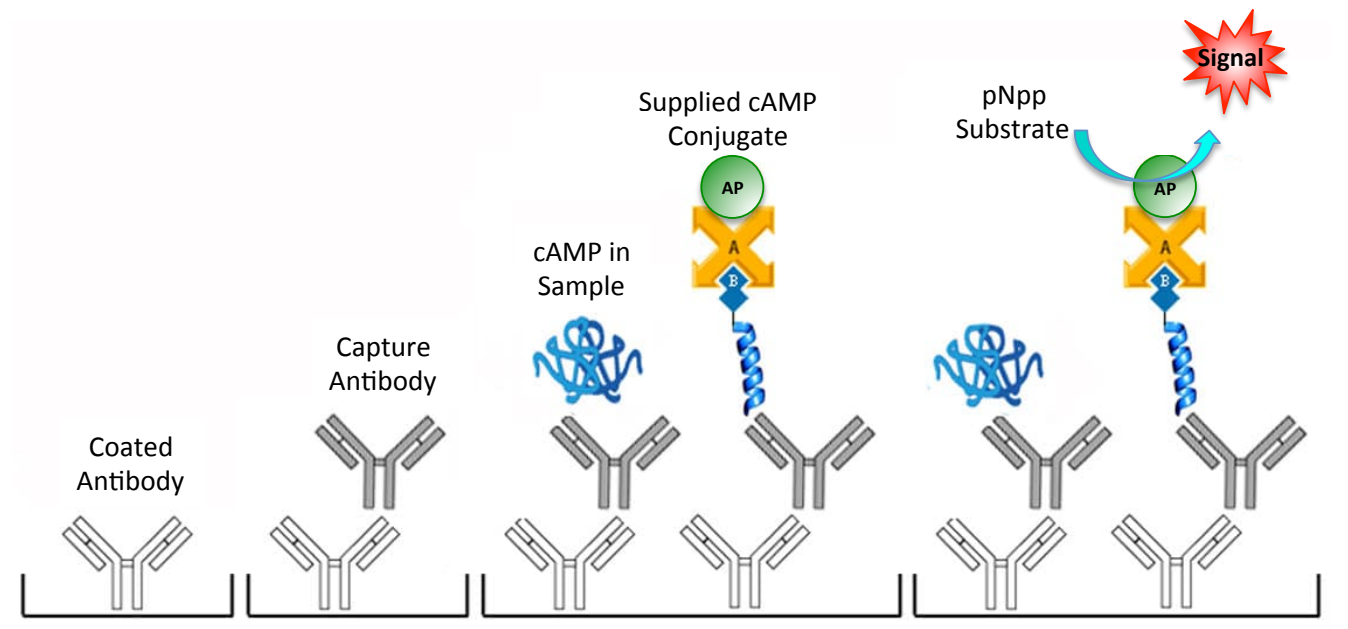

Figure 2.6. Schematic diagram showing the principle of competitive cAMP immunoassay.

Clear supernatants were collected after centrifugation of this homogenate (at $10,000 \mathrm{rpm}$ or $9391 \mathrm{xg}$ for $10 \mathrm{~min}$, at $4^{\circ} \mathrm{C}$ ). After that, neutralizing reagent, $0.1 \mathrm{M}$ $\mathrm{HCl}$, blue conjugate and yellow antibody was added to different wells as mentioned by the manufacturer (fig.2.8, Enzo). Samples along with the reagents were 
pipetted on the microwell plate and then mixed in a shaker for 2 hours at room temperature. After repeated washing steps, the substrate (p-nitrophenyl phosphate) was added to each well to initiate the color reaction catalyzed by alkaline phosphatase. Stop solution was added 1 hour after adding the substrate. The plate was then directly used to measure the absorbance of light at $405 \mathrm{~nm}$ to quantify the amount of color formed, which is indirectly proportional to the amount of cAMP in the tissue sample. The actual amount of cAMP was then calculated by using a standard curve generated from known cAMP concentrations (fig.2.7).

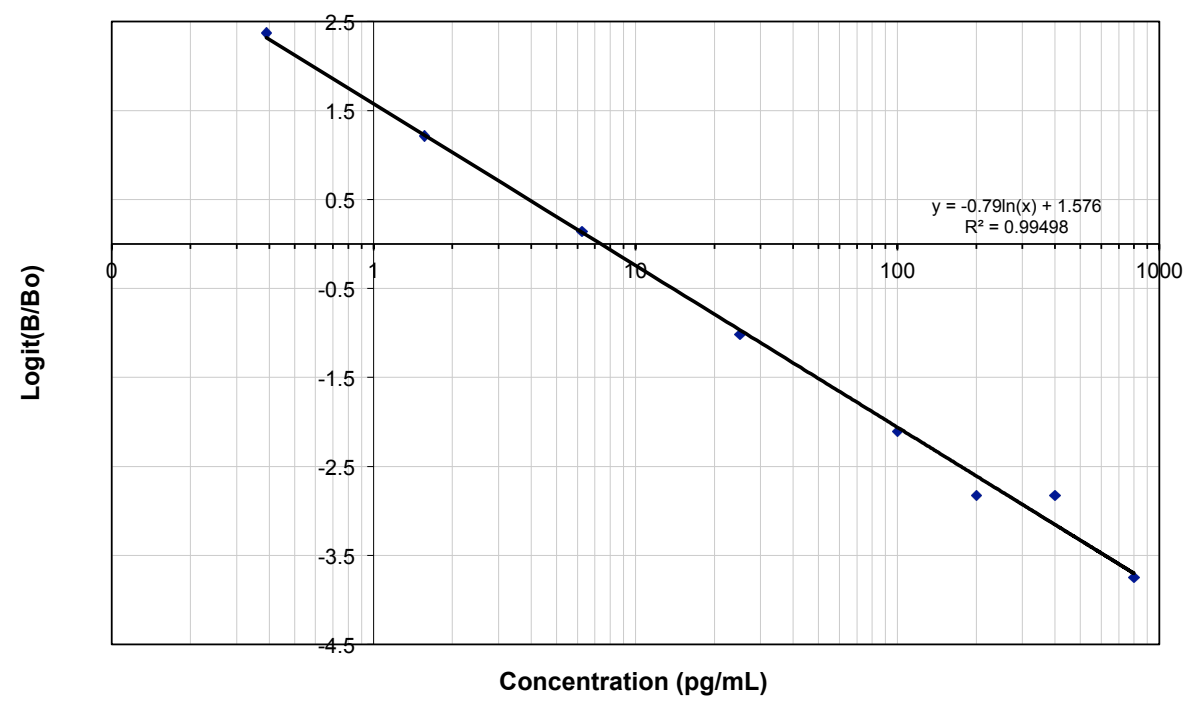

Figure 2.7. The standard curve of supplied cAMP molecules obtained from immunoassay.

\subsection{In-vitro histology experiments}

Histochemical experiments were performed on $5 \mu \mathrm{m}$ thick paraffin-embedded cross sections of whole hearts from six weeks TAC or AngII mice. Then, cardiac fibrosis and morphology were examined using Masson's trichrome (MTC) and Hematoxylin-Eosin (H/E) staining on these tissue sections. Briefly, for MTC staining, after xylene- and graded alcohol (100-50\%)-mediated deparaffinization, slides were fixed with Bouin's solutions (table.2.12). After washing with $\mathrm{ddH}_{2} \mathrm{O}$, slides were incubated with Weigert's iron working solution followed by Biebrich-Scarlet-Acid-Fuchsin solution. After that, slides were sequentially incubated in phosphomolybdic-phosphotungstic acid solution, aniline blue solution, and acetic acid solution. Finally, slides were dehydrated and fixed with Histomount for later analysis (table.2.11). 
Table 2.11. The composition of solutions used in histochemistry experiments.

\begin{tabular}{|c|c|}
\hline Histochemistry solutions & \\
\hline Bouin's Fixative & $\mathrm{ml}$ \\
\hline Saturated Picric acid & 750 \\
\hline Formalin & 250 \\
\hline Glacial acetic acid & 50 \\
\hline Weigert's Iron Working Solution & $200 \mathrm{ml}$ \\
\hline Hematoxylin & $\begin{array}{c}1 \mathrm{~g} \text { in } 100 \mathrm{ml} 95 \% \\
\text { Ethanol }\end{array}$ \\
\hline $\mathrm{FeCl}_{3}, 29 \%$ solution & 4 \\
\hline $\mathrm{HCl}$ (concentrated) & 1 \\
\hline $\mathrm{H}_{2} \mathrm{O}$ & 95 \\
\hline Biebrich-Scarlet-Acid-Fuchsin & $\mathrm{ml}$ \\
\hline 1\% Biebrich-Scarlett & 90 \\
\hline $1 \%$ Acid-Fuchsin & 10 \\
\hline Glacial acetic acid & 1 \\
\hline Phosphomolybdic-Phosphotungs & ution \\
\hline Phosphomolybdic acid (g) & 5 \\
\hline Phosphotungsten acid (g) & 5 \\
\hline $\mathrm{H}_{2} \mathrm{O}(\mathrm{ml})$ & 200 \\
\hline Anilinblue-Solution & \\
\hline Anilinblue (g) & 2.5 \\
\hline Glacial acetic acid (ml) & 2 \\
\hline $\mathrm{H}_{2} \mathrm{O}(\mathrm{ml})$ & 100 \\
\hline $1 \%$ Acetic acid & $\mathrm{ml}$ \\
\hline Glacial acetic acid & 1 \\
\hline $\mathrm{H}_{2} \mathrm{O}$ & 99 \\
\hline
\end{tabular}

\subsection{In-vivo transverse aortic constriction of mice}

Transverse aortic constriction, TAC, is a common method to provoke pressure overload-induced cardiac hypertrophy and heart failure in mice, which mechanistically mimics human aortic stenosis (Rockman et al., 1991). In this method, a knot is made onto the transverse aorta located between the origin of the right innominate and left common carotid arteries. This initially leads to compensated hypertrophy of the heart, which is often associated with a temporary enhancement of cardiac contractility (Toischer et al., 2010). However, the response to the long-term hemodynamic overload becomes maladaptive, resulting in cardiac dilatation and heart failure.

Mice were anesthetized by intraperitoneal injection of medetomidine $0.5 \mathrm{mg} / \mathrm{kg}$, midazolam $5 \mathrm{mg} / \mathrm{kg}$ and fentanyl $0.05 \mathrm{mg} / \mathrm{kg}$. Then hair remover (Veet, Unilever) was used to shave the fur from the neckline to mid chest level. The mouse was then placed in a supine position on a heating pad in order to maintain body temperature. Anesthetic depth was monitored by observing the respiration rate and the toe-pinch reflex of the mouse. A small skin incision was made above the 
upper thoracic inlet to lift up the thyroid and open the muscle surrounding the trachea. A cut was made to the sternum to find the aortic arch. A double knot was made against a 26G needle between the left and right carotid artery. Muscle and skin were sewed up with a suture to close the opening. Sham-operated mice, in which the aortic arch was exposed, but not ligated, were also examined. After finishing the operation, a subcutaneous injection of atipamezole $2.5 \mathrm{mg} / \mathrm{kg}$, flumazenil $0.5 \mathrm{mg} / \mathrm{kg}$ and buprenorphine $0.05-0.1 \mathrm{mg} / \mathrm{kg}$ was applied to each mouse to antagonize the anesthesia and maintain analgesia. Moreover, pain management was controlled by daily treatment with $1.33 \mathrm{mg} / \mathrm{ml}$ metamizole in the drinking water starting two days before the surgery, which continued for seven consecutive days. Three days after surgery, the pressure gradient in the transverse aorta was determined using pulsed wave Doppler imaging.

Echocardiography was performed at three different time points: before the surgery, at two and six weeks after the surgery. In addition, after six weeks of TAC, few mice underwent programmed electrical stimulation (see below) or in vitro electrophysiological experiments. Hearts were also harvested at this time point (after six weeks of TAC) for further processing. During tissue harvest, hearts were rapidly excised and weighed, after that atria and ventricles were snap frozen at $-80^{\circ} \mathrm{C}$. Furthermore, tibia length and wet lung weight were also collected.

\subsection{In vivo osmotic minipump implantation}

The ALZET minipumps from DURECT Corporation are working by osmotic displacement. It has three concentric layers: a rate-controlling semipermeable membrane, an osmotic layer, and an impermeable drug reservoir. Water enters to the pump across the outer, semipermeable membrane due to the presence of a high concentration of sodium chloride in the osmotic chamber. The entry of water causes the osmotic chamber to expand, thereby compressing the flexible reservoir and delivering the drug solution through the delivery portal (Theeuwes and Yum, 1976).

In this project, Alzet osmotic minipumps (OMP), model 2002, were used to continuously infuse Angiotensin II (AngII) (1 $\mathrm{mg} \mathrm{kg}^{-1} \mathrm{day}^{-1}$ ) to mice for 14 days. Mice were pre-weighted to calculate the amount of AngII necessary for 14 days peri- 
od. It was also assumed that each mouse gains 1-2g of weight each week to adjust the AngII dose. The calculated amount of AngII was diluted in saline and inserted to each pump with a filling tube. Flow moderators were carefully introduced, and the pumps were maintained in upright position until implanted into to the mice. The weights of pumps were measured before and after the filling to calculate the percentage of filling. Pumps filled with $95-100 \%$ of solution were then used for implantation.

A mouse was anesthetized by exposing it to $2-4 \%$ isoflurane (gas chamber), and anesthesia was maintained by facemask ventilation of 1-2\% isoflurane in oxygen to insert the pump. Body temperature was maintained at $37^{\circ} \mathrm{C}$ using a thermal pad. The mouse was then prone positioned. After removing hair from the neck, a small cut was made close to neck to insert the pump subcutaneously. Once the pump was inserted, the cut was fixed with suture. After surgery, ventilation was stopped, and facemask was removed carefully to allow the mouse breath normally. The mouse was monitored at least one hour to ensure its full recovery from anesthesia. As a control, OMP containing saline was inserted into another set of mice. All these mice were grouped and followed either two or six weeks of the implantation. During this time, heart function was monitored via echocardiography before and two weeks after the surgery. After two or six weeks, respectively, mice were either used for programmed electrical stimulation or directly sacrificed for protein or histology or other in vitro electrophysiological analysis.

\subsection{Blood pressure measurement}

The CODA mouse rat tail-cuff system (Kent Scientific), which uses volumepressure recording (VPR) sensor technology, was used to measure the mousetail blood pressure. The mouse was anesthetized with $2 \%$ isoflurane in 1.0 $\mathrm{L} / \mathrm{min} 100 \% \mathrm{O}_{2}$ and inserted into a clear acrylic tube with nose cone holder (Kent Scientific) to restrict its movements and to avoid artifacts from the cuff measurements. After that, the tube containing the mouse was mounted onto a heating plate, so that mouse could wake up and get used to with the tube. After its acclimatization to the tube for at least half an hour, the cuff was mounted on the mouse-tail. Initial readings, at least first 15 minutes of recording, were dis- 
carded to get more accurate measurements. Minimums of 20 recordings of systolic and diastolic blood pressure were averaged for each mouse. After measurement, the mouse was released from the small tube and monitored for an additional hour until it returned to the normal physiologic condition.

\subsection{In vivo echocardiography experiments}

Echocardiography was performed on an anesthetized mouse (1.5-2\% isoflurane maintained by face mask ventilation) at $37^{\circ} \mathrm{C}$ (heating plate, anal probe for feedback temperature control). After removal of fur by hair removing cream (Veet, Unilever), the ultrasound transducer (30 MHz center frequency) was placed on the chest to record M-mode and B-mode images and loops at parasternal long and short axis (Vevo 2100 system, Visualsonics) (fig. 2.12). Original B-mode data were used to analyze with Vevo Lab software. In this parasternal short axis view both papillary muscles were used for proper positioning of the mouse heart under transducer. After that, built-in indicators were applied to mark epi- and endo-cardium in both systole and diastole to calculate functional parameters (table.2.12). Moreover, length and diameter of the heart were also calculated from B-mode data. Once echocardiography was done, then the mouse was undergone other experimental procedures (like in vivo EPU experiments or survival analysis; in vitro electrophysiology experiments or western blot experiments).

Table 2.12. Representative echocardiographic parameters and their calculation formulas.

\section{Functional parameters:}

Fractional Area Shortening, \% Fractional Shortening, \% Volume in Systole, $\mu \mathrm{l}$

Volume in Diastole, $\mu \mathrm{l}$

Ejection Fraction, \% Stroke volume, $\mu \mathrm{l}$ Cardiac Output, $\mu \mathrm{l} / \mathrm{min}$ Cardiac index, $\mu \mathrm{l} / \mathrm{min} / \mathrm{kg}$

$\begin{array}{cl}\text { FAS } & \text { (Area d-Area s)/ Area d x } 100 \\ \text { FS } & \text { (LVID d-LVID s)/ LVID d x } 100 \\ \text { Vol s } & 5 / 6 \times \text { Area s x L s } \\ \text { Vol d } & 5 / 6 \times \text { Area d x L d } \\ \text { EF } & (\text { Vol d-Vol s)/Vol d x } 100 \\ \text { SV } & \text { Vol d-Vol s } \\ \text { CO } & \text { SV x HR } \\ \text { CI } & \text { CO/BW/1000 }\end{array}$

ELABORATION

$\mathrm{d}=$ diastole

$\mathrm{s}=$ systole

LVID= left ventricular inner diameter

$\mathrm{L}=$ length

Vol= volume

$\mathrm{HR}=$ heart rate

BW= body weight

\subsection{In vivo electrophysiological studies (EP studies)}

Programmed electrical stimulation at the right ventricular apex was performed in mice. After the onset of anesthesia, which was further maintained by facemask ventilation using $2 \%$ isoflurane in $1.0 \mathrm{~L} / \mathrm{min} 100 \% \mathrm{O}_{2}$, 27-gauge needle electrodes were subcutaneously placed on each limb of the head back supine posi- 
tioned mice to record the surface ECG. The bio-potentials were amplified and digitized by AD Instruments (Dunedin, New Zealand).

For the EP study, a cervical incision was made at midline. After careful preparation, the right jugular vein was exposed. Following distal ligature, an incision was made in the vein, and a 1.1F octapolar catheter (EPR-800; Millar Instruments) was introduced until its tip lead reached to the right ventricular (RV) apex close to the diaphragm. A proximal ligature was also made to the jugular vein to avoid bleeding. Optimal position was figured out using bipolar RV stimulation (between the tip lead and the lead next to it), which was monitored by simultaneous recording of surface ECG and intracardiac ECG from the remaining electrodes. Electrical changes due to depolarization of heart muscle during each beat show this type of tracing, where P-wave represents atrial depolarization, QRS complex represents ventricular depolarization, T-wave represents ventricular repolarization and U-wave represents papillary muscle repolarization. However, deformation in the pattern or duration of any of the segments represents cardiac abnormalities.

During this EP study, optimal catheter position was considered to be the lowest possible stimulation threshold. Firstly, ventricular pacing threshold was checked by applying $200 \mu \mathrm{A}$ current pulses for $2 \mathrm{~ms}$ with a basic cycle length (BCL) of 88 ms for 50 times to test for the consistency of stimulus capture.

After finding the appropriate position in RV, the simulation was performed by an electronic stimulator to check arrhythmic vulnerability (STG3008-FA, multichannel systems). Ventricular arrhythmias were induced by a decremental burst pacing protocol. Burst pacing started at a $40 \mathrm{~ms}$ cycle length and decreasing by 2 ms in every 2 seconds till a cycle length of $20 \mathrm{~ms}$ (Li and Wehrens, 2010). Burst pacing was repeated, for a total of three times in each mouse, one minute after the previous burst concluded or the termination of VT. VT was defined as the occurrence of rapid ventricular electrocardiograms with different QRS morphology (wider QRS complex) that lasted longer than 1 second. These arrhythmias could be monomorphic (QRS complex morphology does not change) or polymorphic 
(different QRS complex morphologies). If at least one burst or extra stimulation (out of 3) produced VT, the mouse was considered inducible.

\subsection{Data analysis}

All the numerical data are presented as Mean \pm standard error (SEM). Western blot, immunohistochemistry, immunocytochemistry and fluorescent images were analyzed using Image J v1.5 software (NIH). Epi-fluorescence data were analyzed using IonWizard v6.4 (IonOptix). Patch-clamp data were analyzed using PatchMaster v2 (HEKA Electronics). Programmed electrical stimulation data were analyzed using LabChart 7.0 software (AD Instruments). Echocardiographic data were analyzed using Vevolab v1.7 software (VisualSonic). All the data were transferred into Microsoft Excel 2011 and GraphPad Prism v6.0 software for graphical presentation and statistical analysis. For $\mathrm{I}_{\mathrm{Ca}}$ steady-state activation, fits were tested for significant difference using $\mathrm{F}$ tests. For longitudinal data, 2way repeated measures ANOVA was performed; where appropriate, one-way ANOVA with multiple comparison tests (Fishers least significant difference, LSD, test) was used. Otherwise, Student's unpaired t-test was considered. Two-sided $\mathrm{P}<0.05$ was considered as significant. 


\section{Chemicals used in this study}

2-Propanol

2,3-Butanedione monoxime

Albumin fraction $\mathrm{V}$

Ammonium persulfate

Angiotensin II

Antibody diluant

Boric acid

Bromphenol blue sodium salt

Caffeine

Calcium chloride solution

CellROX

Cesium chloride

Cesium hydroxide solution

Coomassie brilliant blue R250

D \pm Glucose

di-Sodium hydrogen phosphate dihydrate

Dimethyl sulfoxide

Direct CAMP ELISA kit

DirectPCR-Tail

dNTPs

DreamTaq Green Buffer (10X)

EDTA

EDTA $\mathrm{Na}_{2} 2 \mathrm{H}_{2} \mathrm{O}$

EGTA

Entellan Histomoun

Ethanol, >99,8\%

Ethidium bromide-solution $1 \%$

Forene

Fura-2-AM

Fluo-4-AM

GeneRuler 100 bp Plus DNA Ladder

Glycero

Glycine

Goat serum

GoTaq DNA Polymerase, 500U

$\mathrm{H}-89$ dihydrochloride hydrate

HEPES

Hydrochloride acid $37 \%$

L-Glutamic acid

Laminin

LE Agarose

Liberase DH

Magnesium chloride

Maleimide

Methanol

MitoSOX Red

$\mathrm{N}, \mathrm{N}, \mathrm{N}^{\prime}, \mathrm{N}^{\prime}$-Tetramethylethylenediamine

Phenol red sodium salt

Phenylmethanesulfonyl fluoride

PhosStop

Pierce BCA Protein Assay Kit

Ponceau S

Potassium bicarbonate

Potassium chloride

Potassium phosphate monobasic

Protease Inhibitor Cocktail

Protein Marker V

Reblot plus srong Antibody Stripping Solution Roti-Histofix 4\%

Rotiphorese Gel 30

Shandon-Harris Hematoxylin

Sodium bicarbonate

Sodium chloride

Sodium dihydrogen phosphate monohydrate

Sodium dodecyl sulfate

Sodium fluoride

Sodium hydroxide

Sodium hypochlorite solution

Sodium orthovanadate

Sodium phosphate monobasic dihydrate

Sodium sulfate

Taurine

Tetraethylammonium chloride

Tetramethylammonium chloride

TRIS

Tris-Glycine-SDS buffer, 10x

Triton-X 100

Trypsin 2,5\%

Tween-20

Vectashield mounting medium

$\beta$-Mercaptoethanol
MERCK Millipore, \# 109634

Sigma, \# B0753

Roth, \# 8076.4

Roth, 9178.1

Sigma, \# A9525

Dako, \# S0809

Roth, \# 6943.1

Applichem, \# A1120

Sigma, \# C0750

Sigma, \# 21115

life technologies, \# C10443

Sigma, \# C3032

Sigma, \# 232041

MERCK, \# 112553

Roth, \# HN06.3

MERCK Millipore, \# 119753

Sigma, \# 41640

Enzo Life Sciences, \# ADI-900-066

Peqlab, \# 31-102-T

Promega, \# U1240

ThermoFischer Scientific, \# B71

Roth, \# 8040

Sigma, \# E5134

Sigma, \# E4378

MERCK Millipore, \# 107960

Roth, \# 5054.4

Roth, \# 2218.3

Abbott

Invitrogen, \# F-1201

Invitrogen, \# F-1420

ThermoFischer Scientific, \# SM0324

Sigma, \# G8773

Roth, \# 3908.3

Dako, \# X0907

Promega, \# M3175

Sigma, \# B1427

Roth, \# 9105.3

Roth, \# X942.1

Sigma, \# G1251

Sigma, \# L2020

Biozym, \#840004

Roche, \# 05401054001

Roth, \# KK36.3

Sigma, \# 129585

Roth, \# 7342.1

life technologies, \# M36008

Roth, \# 2367.1

Sigma, \# P5530

Sigma, \# 7883

Roche, \# 04906837001

ThermoFischer Scientific, \# 23225

Sigma, \# P3504

Sigma, \# 60339

Sigma, \# P9333

Sigma, \# P5655

Roche, \# 11872580001

Peqlab, \#27-2211

MERCK Millipore, \# 2504

Roth, \# P087.5

Roth, \# 3029.1

ThermoFischer Scientific, \# 23225

Sigma, \# S7920

Roth, \# P029.1

MERCK Millipore, \# 106346

Roth, \# 2326.2

Sigma, \# S7920

Roth, \# 6771.3

Sigma, \# 71696.2

Sigma, \# S6508

Sigma, \# 71505

MERCK Millipore, \# 106649

Sigma, \# T0625

Sigma, \# T2265

Sigma, \# T19526

Roth, \# 5429.3

Bio Rad, \# 161-0772

Sigma, \# X100

Gibco, \#15090-046

Bio Rad, \# 170-65

Vector Laboratories, \# H-1400

Sigma, \# M3148 


\section{Instruments used in this study}

$1.5 \mathrm{ml}$ Eppendorf tubes

$15 \mathrm{ml}$ tubes

$2.0 \mathrm{ml}$ Eppendorf tubes

$50 \mathrm{ml}$ tubes

6-0 polyviolene suture

96 well plates

Biotek reader

C-DiGit blot scanner

Capillary tubues

Centrifuge

ChemiDoc MP system, scanner

Confocal microscope

DEWAR carrying flasks

Discofix-3 three-way valve

Eight-electrode catheter

Electrical stimulus generators

Homogenizer

Homogenizer

Laboratory balances

Light macroscope

Magnetic stirrers and heating Plate

Microscope slides

Microtom Leica RM 2165

Mini Protean TGX gels

Mini-PROTEAN electrophoresis system

MS-400 MicroScan transducer

Nunc Lab-Tek chamber slide

$\mathrm{pH}$ mete

Pipette

PowerLab 16/35

Powerpac HC

Prolene suture 6-0

Prolene suture, taper point, C-1, 30", Size 6-0

Protran nitrocellulose transfer membrane

Pump with independent channel control

Recirculating water bath

Research plus multi-channel pipette

Serological pipettes

Short plates for western blot

Spacer plates for western blot

Sterican 26-gauge needle

Sterican 27-gauge needle

Steriflips

PCR thermocycler

Thermomixer

Trans blot turbo transfer pack

Trans-Blot Turbo transfer system

U-40 Insulin Omnifix Solo

Vevo 2100

Vortex mixer

Water bath

WB power pack universal

Western blot HRP substrate

X-ray film

X-ray film processor SRX 101A

Epifluorescence microscope

PMT filters

Myo Cam S and power adapter

Fluorescence system interface

Myopacer

MyoPacer cell stimulator

Cairn 75W continuous xenon housing and power supply

Temperature control

cFlow V2.x 8-channel switch/flow control system

UPLSAPO 40x objective

Microscope

Console drives

Patch Clamp

Amplifier

Red Star headstage

Microscope

DMZ electrode puller

Thin wall capillary

Bath electrode, $1 \mathrm{~mm}$ Pin with $30 \mathrm{~cm} \mathrm{~L}, 26$ ga. Insulated Wire

$\mathrm{Ag} / \mathrm{AgCl}$ electrode pellets discs

Teflon coated silver wire

MicroFil

O-ring for pipette holder
Eppendorf, \# 0030123.328

Greiner bio-one, \# 188271

Eppendorf, \# 0030120.248

Greiner bio-one, \# 210261

Harvard Apparatus

Nunc, \# 167008

BIOTEK Instruments, \# Powerwave X

Licor, \#3600

Biozym, \# 13060022

Eppendorf, \# 5424R

Biorad

LSM Pascal, Axiovert 200M

KGW isotherm

Braun, \# 4095111

Millar, \# EPR-800

Multichannel systems, \# STG4002

Metabo, \# SBE850

Miccra, \# D-1

Sartorius

Olympus

Heidolph, \# 505-20000-00

Thermo Scientific, \# J1800AMNZ

Leica

Bio Rad, \# 456-1096

Biorad

Linear Array Technology

ThermoFischer Scientific, \# 177399

SI Analytics, \# Prolab1000

eppendorf research plus

AD Insruments

Biorad

Ethicon

Ethicon, \# 8889H

Whatman, \# 4018650

Ismatec, \# Reglo Icc

HAAKE, \# F3

Eppendorf

Eppendorf

Bio Rad, \#1653308

Bio Rad, \#1653311

Braun, \# 16010396E

Braun, \# 16035054E

Millipore, \# SCGP00525

Peqlab, \# peqSTAR

eppendorf, \# Thermomixer comfort

Bio Rad, \# 170-4156

Biorad

Braun, \# 9161309v

VisualSonics

Scientific industries, SI-0256

Julabo

Biorad

MERCK Millipore, \# WBKLS0500

Fujifilm, \# 4014403

Konica

CHROMA

IonOptix

IonOptix

IonOptix

IonOptix

Warner Instruments

Cell MicroControls

Olympus

Motic, AE31

Masterflex

HEKA EPC 10 USB

HEKA

Nikon, \#Diaphot300

Zeitz

WPI, \# TW150F-3

Warner Instruments, \# 64-1328

WPI, \# EP2

WPI, \# AGT1010

WPI, \# MF34G

HEKA, \# MTF011 


\section{Chapter3 Results}


Angiotensin II (AngII) and pressure overload both have been accused of arrhythmia and heart failure (Drake-Holland et al., 2001; Norton et al., 2002; Schillaci et al., 1996; Wang et al., 2014). Scientists have also reported the role of reactive oxygen species in these settings (Tsutsui et al., 2011). Moreover, AngII and pressure overload are also responsible for the production of reactive oxygen species (Dikalov and Nazarewicz, 2013; Schwarzer et al., 2014; Takimoto and Kass, 2007). Here in this thesis, it was hypothesized that AngII- and pressure overload-implicated these pathophysiological changes via the production of ROS, which ultimately altered its downstream targets, like PKA to mediate these pathological outcomes.

\subsection{AngII-induces cytosolic oxidant production}

Upon stressed conditions, cells produce AngII to initiate several downstream signaling to cope up with the stress (Sachse and Wolf, 2007). In order to test, whether this AngII can induce oxidant production on cardiomyocytes, $1 \mu \mathrm{M}$ AngII was added to the isolated ventricular myocytes, which robustly increased cytosolic ROS production as assessed by CellROX in a time-dependent manner (fig.3.1). Interestingly, there was no difference in ROS production between WT and KI cardiomyocytes.
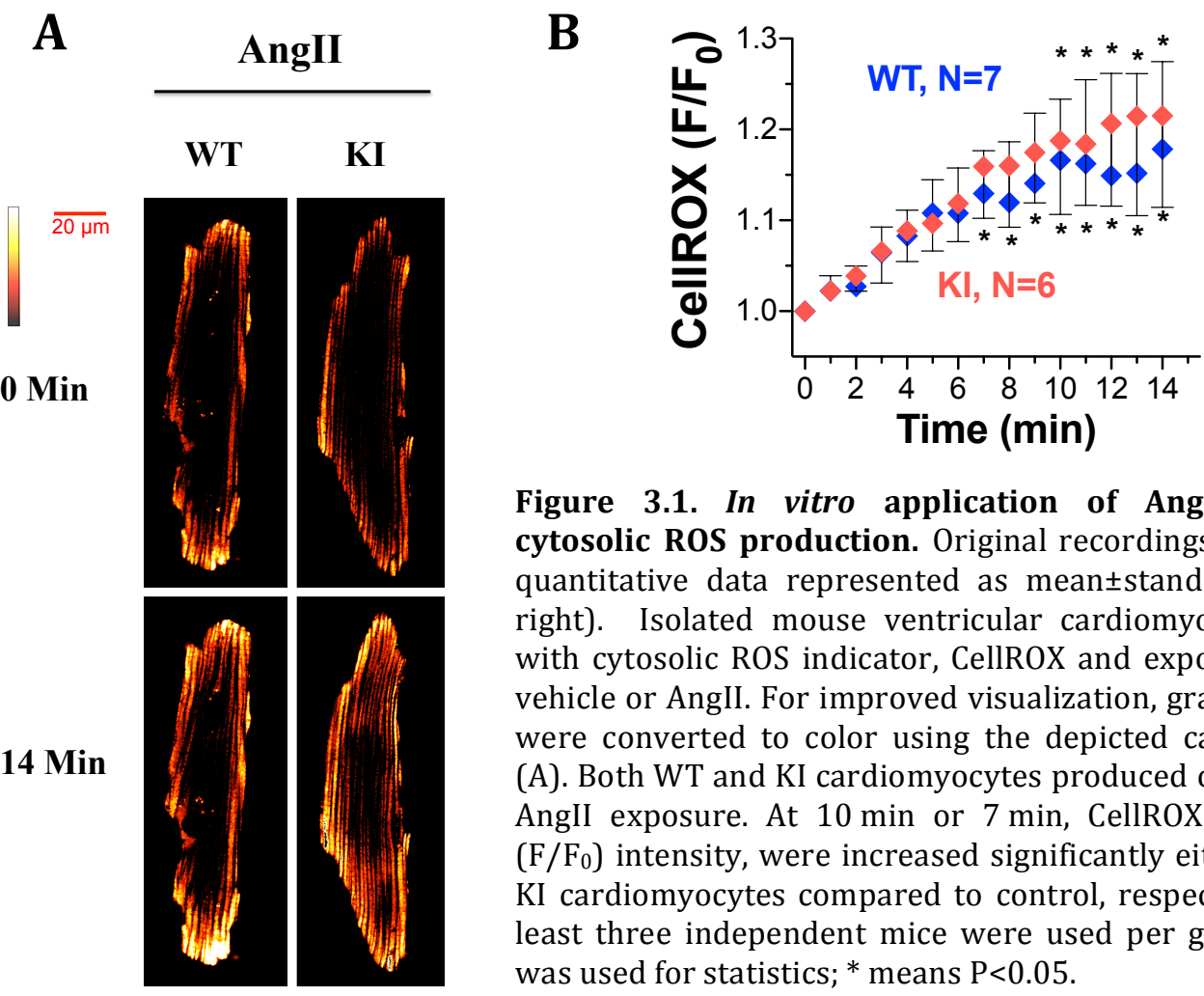

Figure 3.1. In vitro application of AngII increases cytosolic ROS production. Original recordings (A, left) and quantitative data represented as mean \pm standard error $(B$, right). Isolated mouse ventricular cardiomyocytes loaded with cytosolic ROS indicator, CellROX and exposed to either vehicle or AngII. For improved visualization, grayscale values were converted to color using the depicted calibration bar (A). Both WT and KI cardiomyocytes produced oxidants upon AngII exposure. At $10 \mathrm{~min}$ or $7 \mathrm{~min}$, CellROX fluorescence $\left(\mathrm{F} / \mathrm{F}_{0}\right)$ intensity, were increased significantly either in WT or KI cardiomyocytes compared to control, respectively (B). At least three independent mice were used per group; ANOVA was used for statistics; * means $\mathrm{P}<0.05$. 
Immediately after AngII application, WT cardiomyocytes started to produce ROS. Compared to baseline $(0 \mathrm{~min})$, CellROX fluorescence became significantly increased $7 \mathrm{~min}$ after initial exposure to AngII (to 1.13 \pm 0.05 , fig.3.1). Similarly, CellROX fluorescence gradually increased in KI with a significant difference to baseline at $10 \mathrm{~min}(1.19 \pm 0.08$, fig.3.1). There was no significant difference in the CellROX signal between WT and KI upon AngII.

\subsection{AngII-induces mitochondrial oxidant production via NOX2 enzyme}

Since it is known that increased amount of cytosolic ROS can diffuse into mitochondria to stimulate mitochondrial ROS production by a ROS-induced ROS release mechanism, mitochondrial ROS production was tested using MitoSOX. In order to test the effect of AngII on mitochondrial ROS production, isolated ventricular cardiomyocytes were exposed to AngII in the presence of MitoSOX. External application of $1 \mu \mathrm{M}$ AngII to isolated WT and KI ventricular cardiomyocytes resulted in a time-dependent increase in MitoSOX fluorescence (fig.3.2).
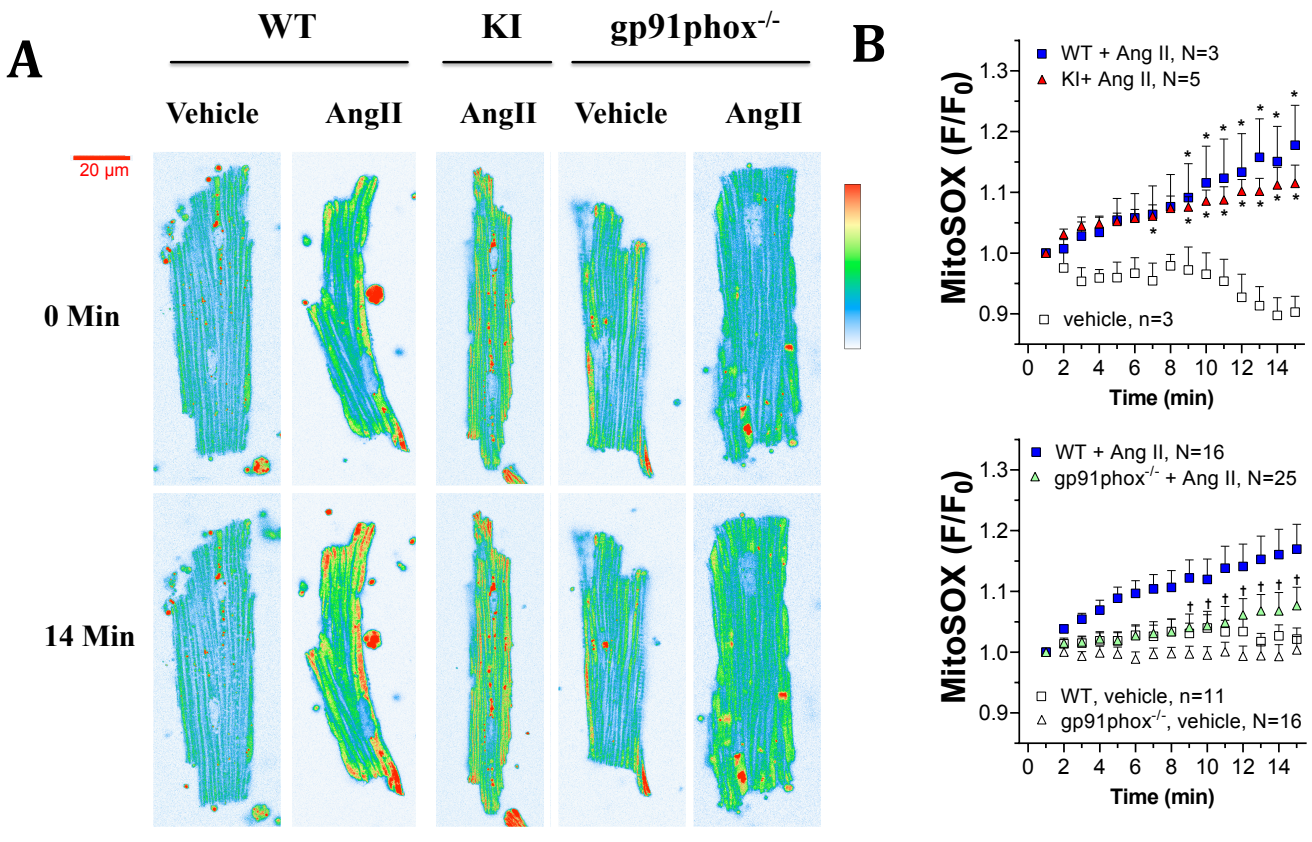

Figure 3.2. AngII-mediated mitochondrial ROS production. Original recordings (A, left) and quantitative data represented as mean \pm standard error (B, right). Isolated mouse ventricular cardiomyocytes loaded with mitochondrial ROS indicator, MitoSOX and exposed to either vehicle or AngII. For improved visualization, grayscale values were converted to color using the depicted calibration bar (A). Both WT and KI cardiomyocytes produced oxidants upon AngII exposure. At 9 min or $7 \mathrm{~min}$, MitoROX fluorescence $\left(\mathrm{F} / \mathrm{F}_{0}\right)$ intensity was increased significantly either in WT or KI cardiomyocytes compared to control, respectively (B). In gp91phox K0 cardiomyocytes, AngII could not produce ROS (bottom, right). At least three independent mice were used per group; ANOVA was used for statistics; * means $\mathrm{P}<0.05$. 
In WT, compare to vehicle-treated cardiomyocytes, AngII caused a significant increase in MitoSOX fluorescence as early as $9 \mathrm{~min}$ after initial exposure (Vehicle vs. Ang II, $0.97 \pm 0.04$ vs. $1.09 \pm 0.06$, fig.3.2). In KI, MitoSOX fluorescence started to be significantly increased at 7 min (Vehicle vs. AngII, $0.97 \pm 0.04$ vs. $1.06 \pm 0.02$, fig.3.2). Interestingly, this AngII-dependent increases in MitoSOX fluorescence was completely abolished in cardiomyocytes lacking the catalytic subunit of NADPH oxidase 2 (gp91phox knockout mice), suggesting that sarcolemmal NOX2-dependent ROS production is crucially involved (WT AngII vs. gp91phox KO AngII, $1.12 \pm 0.03$ vs. $1.04 \pm 0.02$, fig.3.2).

\subsection{Oxidative activation of type I PKA}

Brennan et al. showed that oxidants could induce an intermolecular dimer formation and activate type I PKA that ultimately phosphorylates downstream targets (Brennan et al., 2006). To test, whether AngII-dependent ROS are indeed capable of oxidizing PKA RI and could induce dimer formation, an in vitro dimer assay was performed.
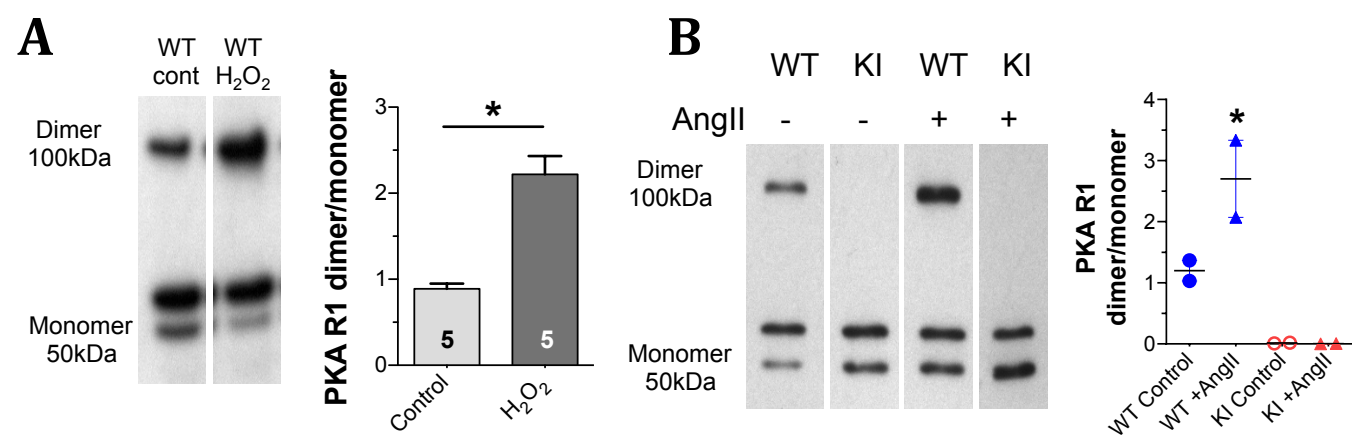

Figure 3.3. Oxidant-induced PKA RI dimer formation. Original recordings (A, left) and quantitative data represented as mean \pm standard error (B, right). In the presence of $\mathrm{H}_{2} \mathrm{O}_{2}$, PKA RI subunit of isolated mouse ventricular cardiomyocytes got oxidized ( $\mathrm{A}$, left) and form significantly higher intermolecular disulfide bond compared to control (A, right). Upon AngII perfusion to isolated whole heart, WT produced significantly higher PKA RI disulfide bonds compared to control (B, left). Interestingly KI mice, which had a cys17ser mutation, could not form the disulfide upon AngII perfusion (B, right). At least two independent mice were used per group; $t$ test and ANOVA were used for statistics, * means $\mathrm{P}<0.05$.

$\mathrm{H}_{2} \mathrm{O}_{2}$ is one of the robustly produced cytosolic ROS, which upon metabolism generates other ROS as well (superoxide, hydroxyl radicals, etc.) (Burdon, 1995). Figure 3.3 shows in WT cardiomyocytes; RI dimer formation was increased in the presence of $\mathrm{H}_{2} \mathrm{O}_{2}(100 \mu \mathrm{M})$. Densitometric analysis revealed that $\mathrm{H}_{2} \mathrm{O}_{2}$ mediated oxidation causes an increase in RI dimer/RI monomer ratio from 
$0.89 \pm 0.06$ to $2.22 \pm 0.21, \mathrm{P}<0.05$ (fig.3.3). Similar effects were observed from Langendorff-hearts perfused with AngII (1 $\mu \mathrm{M}, 10 \mathrm{~min}$, fig.3.3). AngII increased $\mathrm{RI}$ dimer/monomer ratios from $1.20 \pm 0.17$ to $2.70 \pm 0.63, \mathrm{P}<0.05$, fig.3.3). In contrast, no RI dimer formation was observed in KI mice exposed to AngII.

\subsection{Oxidation of PKA results in PKA-dependent LTCC phosphor- ylation}

Among all the PKA substrates of EC coupling, LTCC is critical because of its role in initiating EC coupling and also due to its role in maintaining the plateau phase of action potential. Moreover, scientists reported that LTCC could be the substrate of type I PKA rather than type II (Burton et al., 1997; Jones et al., 2012). In order to test, whether oxidized type I PKA phosphorylates the alpha subunit of LTCC (Cav1.2), I exposed Langendorff-perfused hearts to AngII (1 $\mu \mathrm{M})$ for 10 $\min$.

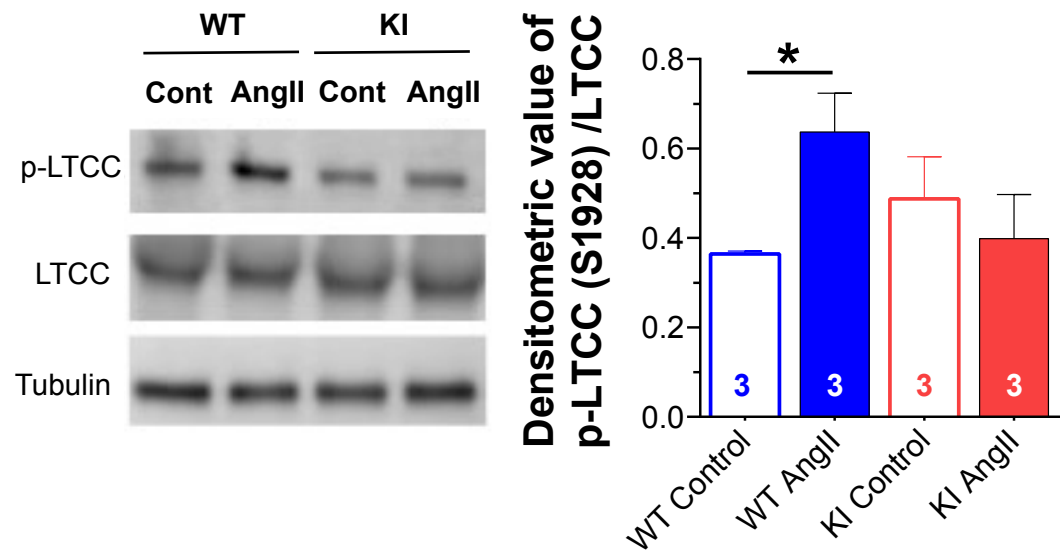

Figure 3.4. AngII-dependent LTCC phosphorylation is mediated by oxidative PKA activation. Original recordings (left) and quantitative data represented as mean \pm standard error (right). Whole heart perfusion with AngII-induced substrate phosphorylation at PKA site, as shown by the quantitative data. In WT, LTCC phosphorylation increased compared to KI upon AngII. At least three independent mice were used per group; ANOVA was used for statistics; * means $\mathrm{P}<0.05$.

Western blot analysis of ventricular tissue of these Langendorff-perfused hearts using a phospho-specific antibody revealed that Cav1.2 phosphorylation at serine 1928 is increased in the presence of AngII (fig.3.4). AngII increased densitometric values for $\mathrm{p}$-LTCC/LTCC from $0.36 \pm 0.01$ to $0.64 \pm 0.09, \mathrm{P}<0.05$. This increase was completely absent in KI cardiomyocytes (control vs. AngII, $0.49 \pm 0.09$ vs. $0.40 \pm 0.10$ ). 


\subsection{AngII-mediated cellular translocation of PKA RI subunits}

PKA type I is mostly located in the cytosol of the cardiomyocyte. However, upon stimulation, it can translocate to the membrane and nuclear compartments (Brennan et al., 2006). To test, whether AngII stimulation results in PKA type I translocation, immunocytochemical staining was performed on fixed mouse cardiomyocytes under basal conditions and upon exposure to AngII (1 $\mu \mathrm{M}, 10 \mathrm{~min})$. Figure 3.5 shows the subcellular distribution of PKA RI. Compared to baseline, AngII stimulation resulted in a significant increase in membrane-associated PKA RI. Region of interest (ROI) analysis of fluorescence intensity at T-tubules (TT), intercalated disc (ICD) and nucleus revealed that AngII significantly increased fluorescence intensity compared to baseline (fig.3.5) in these ROI suggesting increased association with possible target proteins. Quantitative data for ROIs for vehicle vs. AngII are: $1.25 \pm 0.09$ vs. $1.85 \pm 0.11$ (TT), $2.15 \pm 0.20$ vs. $3.26 \pm 0.18$ (ICD) and $0.74 \pm 0.12$ vs. $1.50 \pm 0.16$ (nucleus, all $\mathrm{P}<0.05$, fig. 3.5 ).

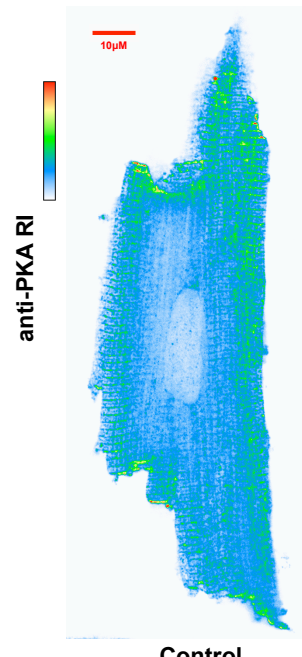

Control
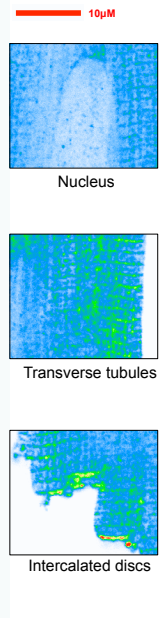

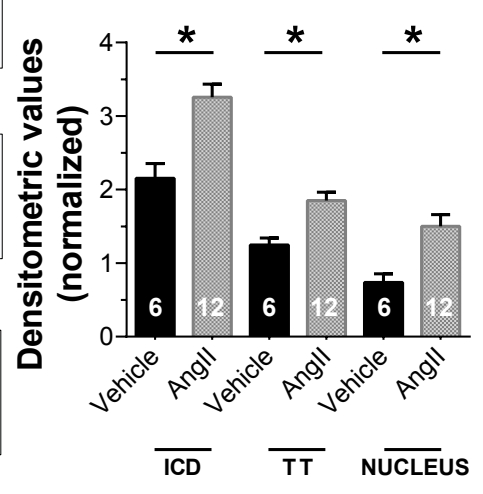

Figure 3.5. Oxidant-induced translocation of PKA RI subunit. Original recordings (A, left) and quantitative data represented as mean \pm standard error (B, right). Isolated mouse ventricular cardiomyocytes, in the presence of AngII $(1 \mu \mathrm{M})$, showed significantly higher membrane and nuclear translocation of PKA RI subunit. For improved visualization, grayscale values were converted to color using the depicted calibration bar. Mean data showed that, upon AngII, PKA RI predominantly translocate to the intercalated disc (ICD), T-tubules (TT) and the nucleus from the cytosol, where, PKA RI typically located. At least three independent mice were used per group; ANOVA was used for statistics; * means $\mathrm{P}<0.05$.

\subsection{Oxidized PKA RI is involved in AngII-dependent regulation of calcium signaling}

In order to test whether oxidized PKA type I is involved in the regulation of excitation-contraction coupling, $\mathrm{Ca}^{2+}$ transients of electrical stimulated $(0.5$ to $3 \mathrm{~Hz}$ ) 
Fura-2-loaded isolated ventricular cardiomyocytes was measured. In WT cardiomyocytes, exposure to AngII $(1 \mu \mathrm{M}, 10 \mathrm{~min})$ did not alter $\mathrm{Ca}^{2+}$ transient amplitude, or Ca transient decay characteristics compared to control (fig.3.6). Interestingly, exposure of AngII to KI cardiomyocytes resulted in significantly reduced $\mathrm{Ca}^{2+}$ transient amplitudes at all investigated stimulation frequencies (fig.3.6).

A

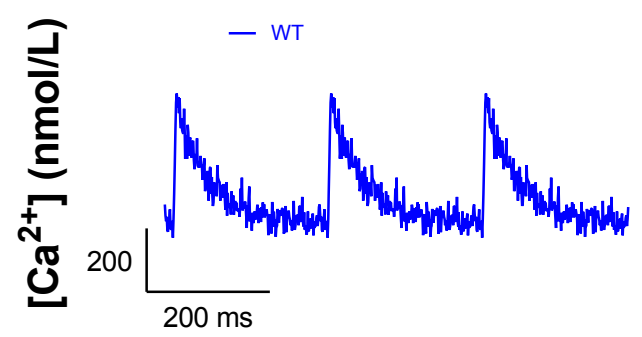

B

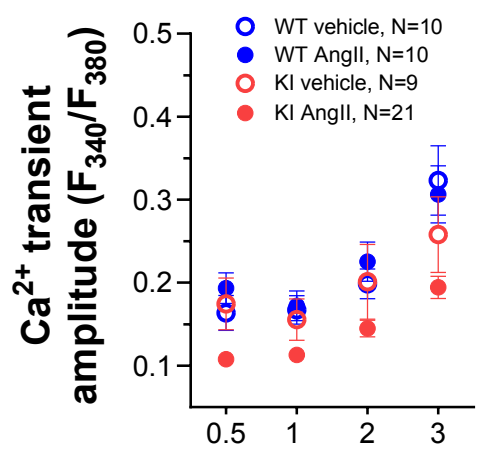

Frequency $(\mathrm{Hz})$

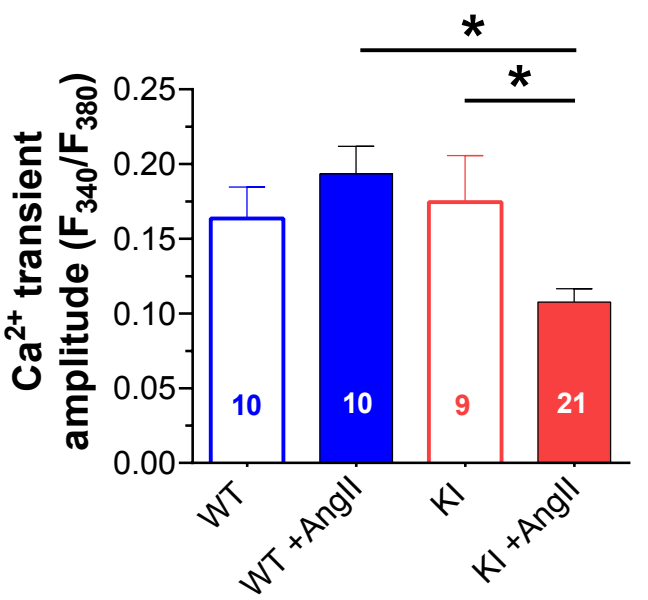

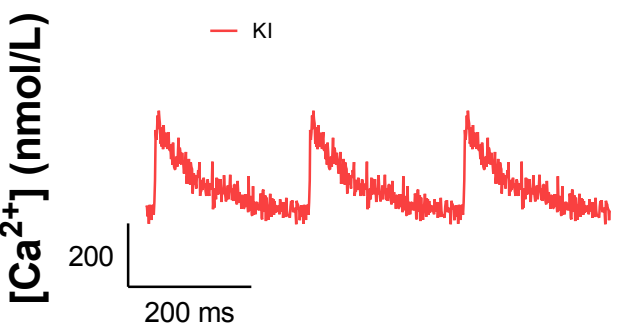

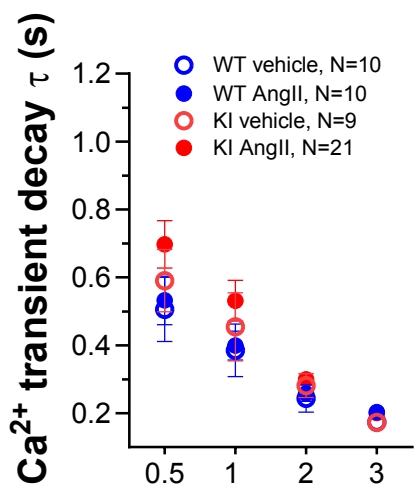

Frequency $(\mathbf{H z})$

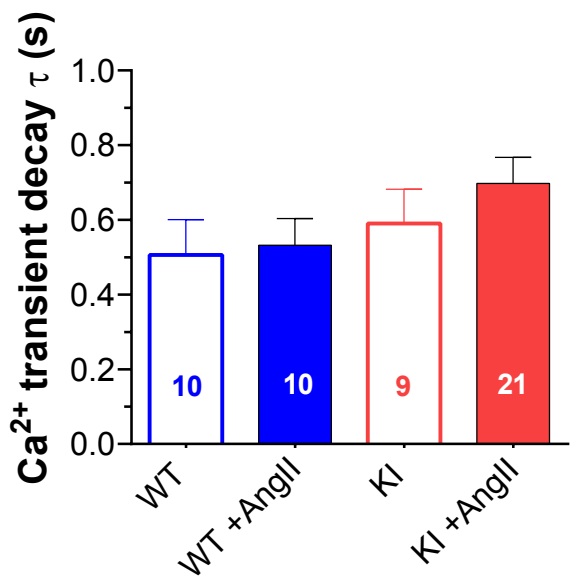

Figure 3.6. AngII maintains $\mathrm{Ca}^{2+}$ transients via oxidative PKA activation. Original data on top (A) and quantitative data represented as mean \pm standard error on the bottom panel (B). Original traces showing baseline calcium transients from WT and KI mice. Fura-2-loaded WT and KI cardiomyocytes were stimulated with different beating frequencies to evaluate their contractile ability (B, top). In the presence of AngII $(1 \mu \mathrm{M})$, WT cardiomyocytes can maintain $\mathrm{Ca}^{2+}$ transient amplitude compared to control (B, below, at $0.5 \mathrm{~Hz}$ ). Interestingly, KI mice could not maintain $\mathrm{Ca}^{2+}$ transient amplitude upon AngII. Moreover, KI took more time to reuptake the released $\mathrm{Ca}^{2+}$ from cytosol back to SR, compared to WT littermates. At least three independent mice were used per group; ANOVA was used for statistics; * means $\mathrm{P}<0.05$. 
Rapid caffeine application technique $(10 \mathrm{mM})$ was then used to induce complete $\mathrm{SR} \mathrm{Ca}^{2+}$ release, to further investigate the underlying mechanism of this reduced $\mathrm{Ca}^{2+}$ transient in KI upon AngII. The resulting caffeine-induced $\mathrm{Ca}^{2+}$ transient can be used as a measure of SR $\mathrm{Ca}^{2+}$ content. Interestingly, AngII dramatically reduced caffeine-induced $\mathrm{Ca}^{2+}$ transients in both WT and KI cardiomyocytes (fig.3.7). While there was a small difference in caffeine-transient amplitude between WT and KI cardiomyocytes at baseline (WT vs. KI; $1.41 \pm 0.11$ vs. $0.94 \pm 0.14, \mathrm{P}<0.05$ ), no difference was observed in the AngII-dependent reduction ( $0.43 \pm 0.06$ vs. $0.35 \pm 0.09$, fig.3.7). After correcting the baseline differences, it was found that AngII reduces caffeine-transient amplitude in WT and KI to a similar extent (fig.3.7). Therefore, reduced SR $\mathrm{Ca}^{2+}$ content cannot explain the difference in the AngII-stimulated $\mathrm{Ca}^{2+}$ transient amplitudes between WT and KI cardiomyocytes.
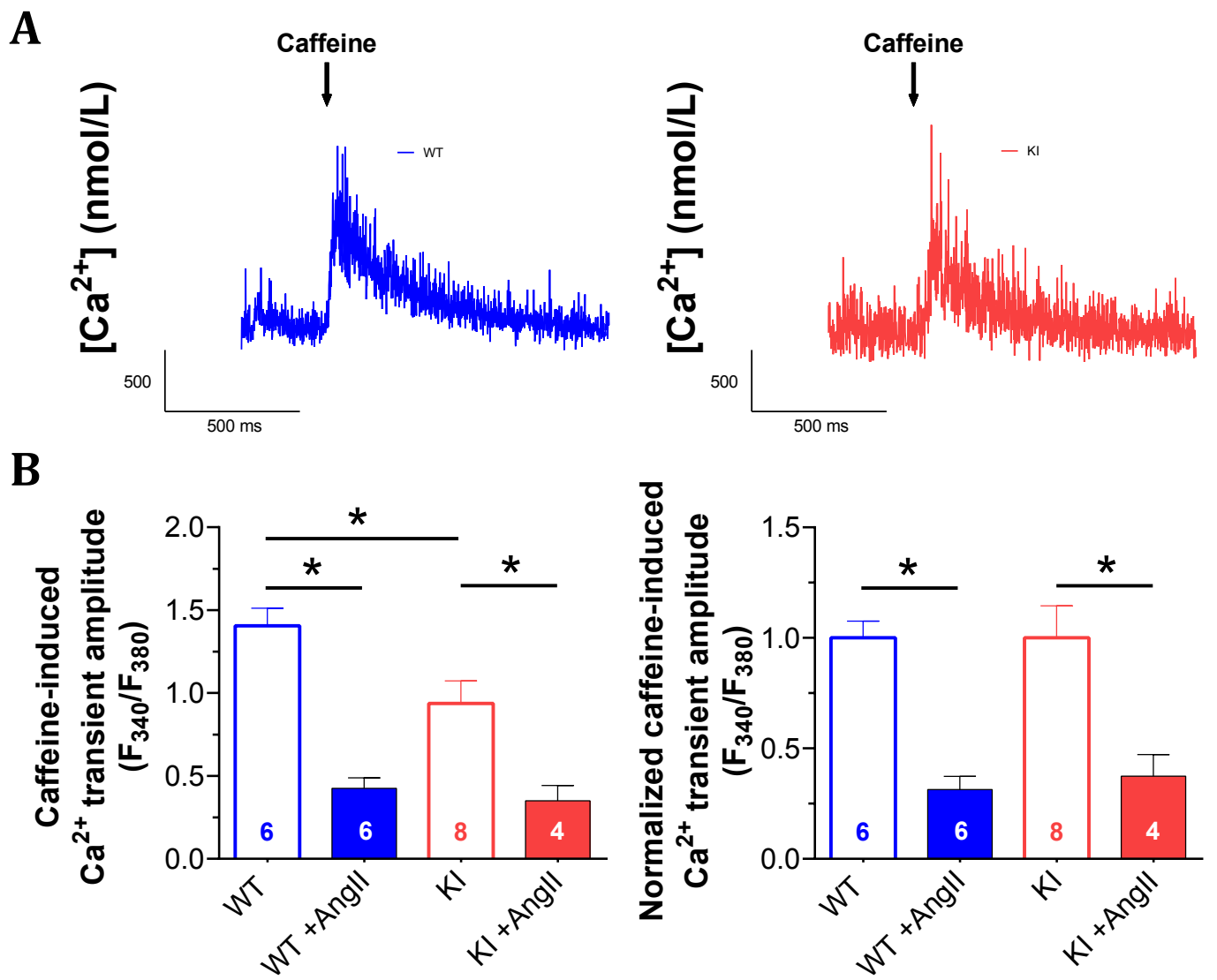

Figure 3.7. Reduction of SR $\mathrm{Ca}^{2+}$ contents upon AngII. Original data on top (A) and quantitative data represented as mean \pm standard error on the bottom panel (B). Fura-2-loaded WT and KI cardiomyocytes were stimulated with $0.5 \mathrm{~Hz}$ beating frequency to evaluate their SR $\mathrm{Ca}^{2+}$ content. At baseline, $\mathrm{KI}$ had less SR content (A). In the presence of AngII $(1 \mu \mathrm{M}), \mathrm{SR} \mathrm{Ca}^{2+}$ content was significantly reduced in both WT and KI (B, left). This decrease was further visualized upon correcting the baseline $(B$, right). At least three independent mice were used per group; ANOVA was used for statistics; * means $\mathrm{P}<0.05$. 
The RyR2 function was evaluated, to identify the dramatic AngII-dependent reduction in SR $\mathrm{Ca}^{2+}$ content. Because SR $\mathrm{Ca}^{2+}$ content is mainly determined by SR $\mathrm{Ca}^{2+}$ leak through ryanodine receptor and $\mathrm{Ca}^{2+}$ reuptake by SERCA2a. It has been reported that the kinetics of the $\mathrm{Ca}^{2+}$ transient decay in mice directly correlate with SERCA2a function (Bers, 2001). Therefore, $\mathrm{Ca}^{2+}$ transient decay characteristics were investigated by fitting the decay to a monophasic exponential equation. The time constant (tau) of the fit, which is a measure of $\mathrm{Ca}^{2+}$ transient decay, however, was not significantly altered by AngII exposure (fig.3.6), suggesting that increased $\mathrm{SR} \mathrm{Ca}{ }^{2+}$ leak, but not reduced SR $\mathrm{Ca}^{2+}$ reuptake, may account for the reduced $\mathrm{SR} \mathrm{Ca}^{2+}$ content.

Since elementary SR $\mathrm{Ca}^{2+}$ release events $\left(\mathrm{Ca}^{2+}\right.$ sparks) underlie the diastolic SR $\mathrm{Ca}^{2+}$ leak, $\mathrm{Ca}^{2+}$ sparks were measured in isolated ventricular cardiomyocytes loaded with Fluo-4 (10 $\mu \mathrm{M}$, fig.3.8). Original line scans and mean data for $\mathrm{Ca}^{2+}$ spark frequency $(\mathrm{CaSpF})$ revealed that AngII dramatically increased diastolic $\mathrm{Ca}^{2+}$ spark frequency as a measure of SR $\mathrm{Ca}^{2+}$ leak (fig.3.8). Compared to the baseline, CaSpF increased significantly from $0.77 \pm 0.09$ to $1.77 \pm 0.19$ in WT and from $0.81 \pm 0.11$ to $1.81 \pm 0.14$ in KI cardiomyocytes, $\mathrm{P}<0.05$, upon AngII.

A

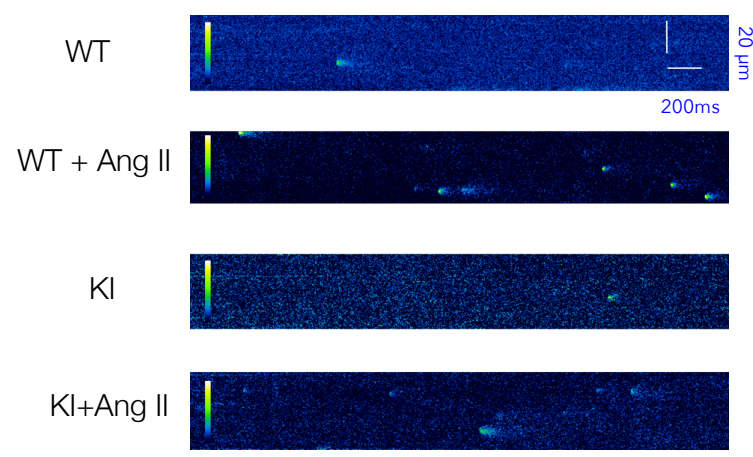

B

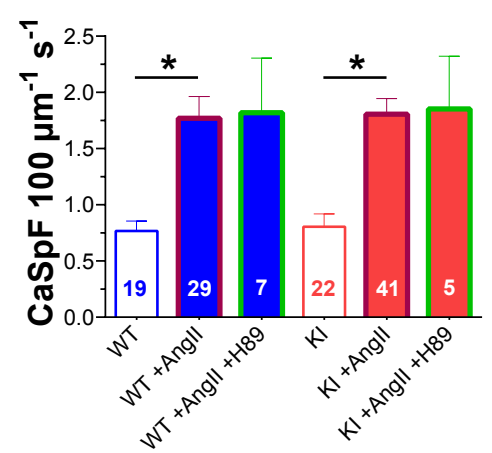

Figure 3.8. Spontaneous $\mathrm{Ca}^{2+}$ release does not depend on PKA oxidation. Fluo-4-loaded WT and $\mathrm{KI}$ cardiomyocytes were stimulated with $0.5 \mathrm{~Hz}$ beating frequencies to evaluate $\mathrm{Ca}^{2+}$ sparks (A, left). At baseline, both WT and KI had similar $\mathrm{Ca}^{2+}$ spark frequencies. In the presence of AngII $(1 \mu \mathrm{M})$, both WT and KI had significantly higher spark frequency (B, right). However, this increase in spark frequency is independent of PKA, as H89 (a PKA inhibitor) could not decrease the spark frequency (B). Quantitative data represented as mean \pm standard error. At least three independent mice were used per group; ANOVA was used for statistics; * means $\mathrm{P}<0.05$.

Interestingly, there was no difference in the AngII-dependent increase in $\mathrm{CaSpF}$ between WT and KI cardiomyocytes. Further evidence for a PKA-independent regulation of $\mathrm{Ca}^{2+}$ leak by AngII is provided by application of the PKA inhibitor 
H89. H89 could not decrease the spark frequency either in WT or KI cardiomyocytes (fig.3.8).

\subsection{Oxidized PKA RI is involved in the regulation of calcium channel gating}

As shown above, that oxidized PKA is involved in Cav1.2 phosphorylation at serine 1928 (see above), which could alter the LTCC-dependent $\mathrm{Ca}^{2+}$ influx $\left(\mathrm{I}_{\mathrm{Ca}}\right)$. Moreover, reduced SR $\mathrm{Ca}^{2+}$ content but unaltered $\mathrm{Ca}^{2+}$ transient amplitude upon AngII exposure may be explained by compensatory increases in $\mathrm{I}_{\mathrm{Ca}}$. Therefore, $\mathrm{I}_{\mathrm{Ca}}$ was measured using whole-cell patch clamp technique (ruptured-patch) in isolated ventricular cardiomyocytes exposed to AngII (fig.3.9).

A
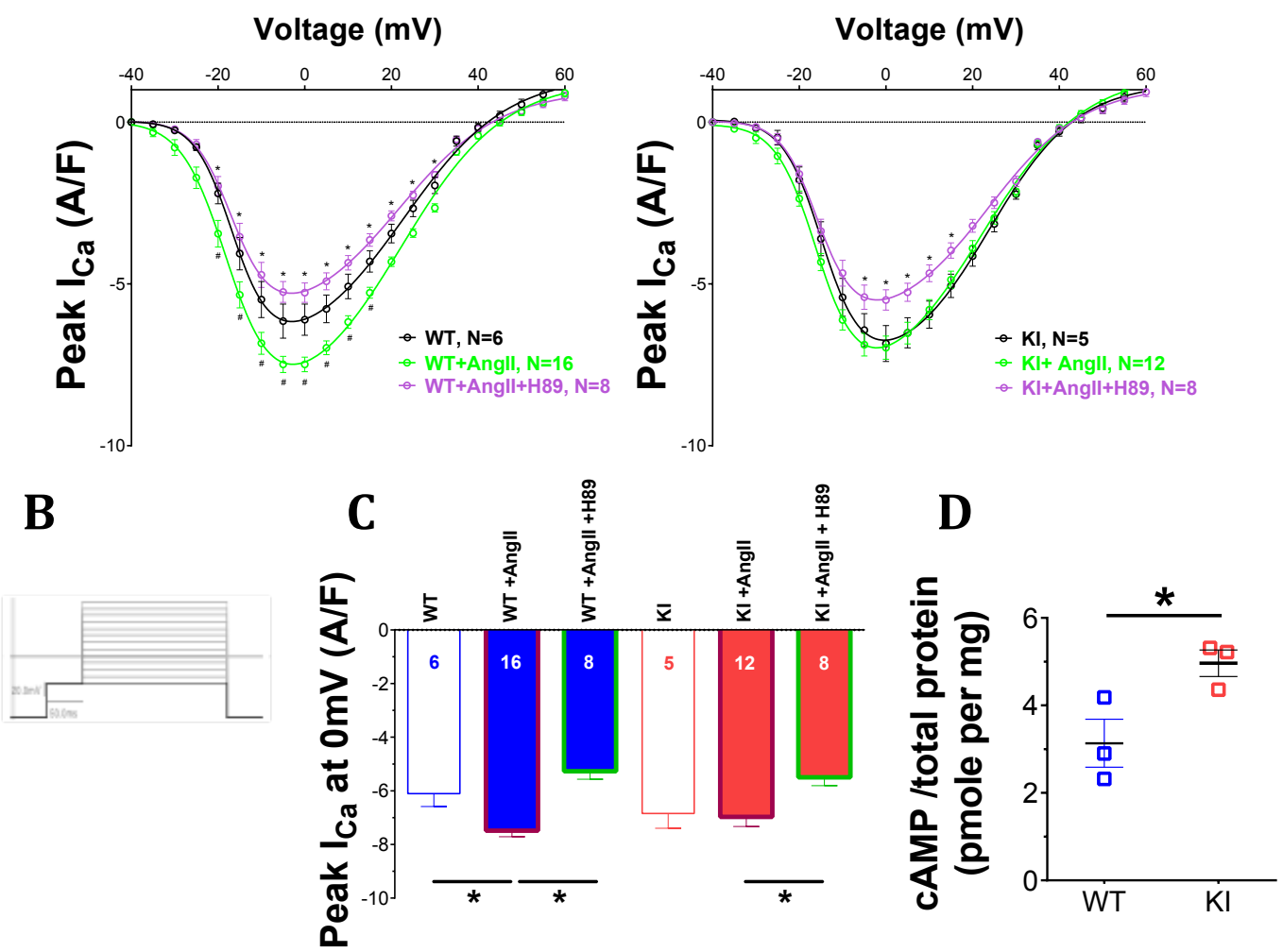

Figure 3.9. AngII increases $I_{C a}$ via PKA oxidation. Whole cell ruptured patch-clamp technique revealed that AngII increased the $\mathrm{I}_{\mathrm{Ca}}$. Current-voltage relationship (A), protocol (B) and peak $\mathrm{I}_{\mathrm{Ca}}$ at $0 \mathrm{mV}(\mathrm{C})$. I-V curve and peak data showed that both WT and KI had comparable peak $\mathrm{I}_{\mathrm{Ca}}$ at baseline, where KI had a tendency of higher $\mathrm{I}_{\mathrm{Ca}}$. Upon AngII, WT could increase the peak. This effect abolished by the use of H89, a PKA inhibitor (A). This AngII-mediated increase was absent in KI. The quantitative mean of $\mathrm{I}_{\mathrm{Ca}}$ at $0 \mathrm{mV}$ showed that AngII could increase $\mathrm{I}_{\mathrm{Ca}}$ and $\mathrm{H} 89$ returned that back to baseline in WT cardiomyocytes. However, in KI, AngII could not increase I $\mathrm{C}_{\mathrm{C}}$; but, H89 decreased $\mathrm{I}_{\mathrm{Ca}}$ from baseline level. D) Interestingly, cAMP levels were increased in KI vs. WT at baseline. Quantitative data represented as mean \pm standard error. At least three independent mice were used per group; ANOVA was used for statistics; in A:\# means $\mathrm{P}<0.05$ vs. vehicle, ${ }^{*}$ means $\mathrm{P}<0.05$ vs. AngII; in C \& D: * means $\mathrm{P}<0.05$. 
Analysis of current-voltage relationship by rectangular voltage steps from -40 to $+80 \mathrm{mV}$ (duration $200 \mathrm{~ms}$ ) revealed that AngII significantly increased peak amplitude $\mathrm{I}_{\mathrm{Ca}}$ in $\mathrm{WT}$ cardiomyocytes (fig.3.9). Peak $\mathrm{I}_{\mathrm{Ca}}$ at $0 \mathrm{mV}$ was $-6.11 \pm 0.48$ vs. $7.47 \pm 0.23 \mathrm{~A} / \mathrm{F}$ (vehicle vs. AngII, $\mathrm{P}<0.05$ ). Interestingly, the PKA inhibitor H89 $(10 \mu \mathrm{M})$ could completely abolish this AngII-dependent increase in I Ia $_{\text {(peak I }}$ at $0 \mathrm{mV}-5.27 \pm 0.30 \mathrm{~A} / \mathrm{F}, \mathrm{P}<0.05$ vs. AngII, fig.3.9). In sharp contrast, AngII exposure did not affect $\mathrm{I}_{\mathrm{Ca}}$ in cardiomyocytes expressing redox-dead type I PKA RI (KI, fig.3.9) suggesting that AngII-dependent impairment of $\mathrm{Ca}^{2+}$ transient amplitude in KI may indeed be due to lack of $\mathrm{I}_{\mathrm{Ca}}$ stimulation. In KI, peak $\mathrm{I}_{\mathrm{Ca}}$ at 0 $\mathrm{mV}$ was $-6.84 \pm 0.55$ vs. $-6.97 \pm 0.36 \mathrm{~A} / \mathrm{F}$ (vehicle vs. Ang II, $\mathrm{p}=\mathrm{N} . \mathrm{S}$.). Interestingly, at baseline, I observed an increased $\mathrm{I}_{\mathrm{Ca}}$ amplitude in $\mathrm{KI}$ cardiomyocytes (compared to WT, fig.3.9), which could be blocked by H89 (fig.3.9) suggesting a greater basal PKA activity in KI. Peak $\mathrm{I}_{\mathrm{Ca}}$ at $0 \mathrm{mV}$ was $-6.33 \pm 0.75 \mathrm{~A} / \mathrm{F}$ in the presence of H89 in KI cardiomyocytes.

cAMP levels were quantitatively measured by colorimetric competitive enzymelinked immunoassay to investigate this baseline difference in calcium channel function. Interestingly, compared to WT, cAMP levels were significantly higher in $\mathrm{KI}$ at baseline ( $3.14 \pm 0.55$ vs. $4.96 \pm 0.30 \mathrm{pmol} / \mathrm{mg}$ of total protein, $\mathrm{P}<0.05$, fig.3.9), suggesting that basal PKA activity may be more in KI cardiomyocytes.

A

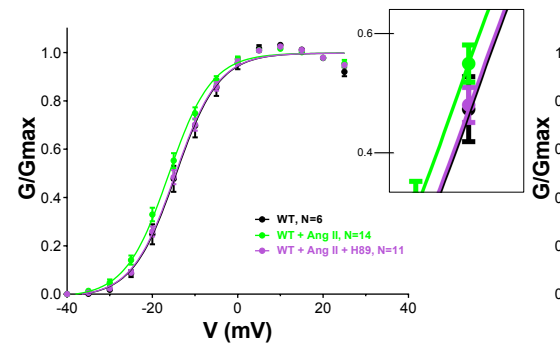

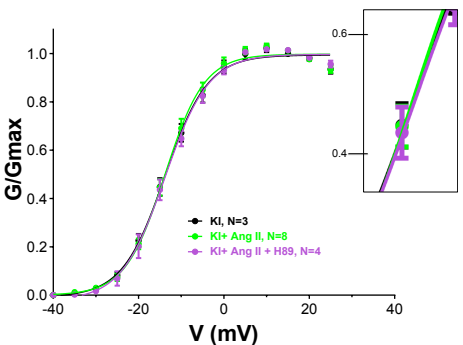

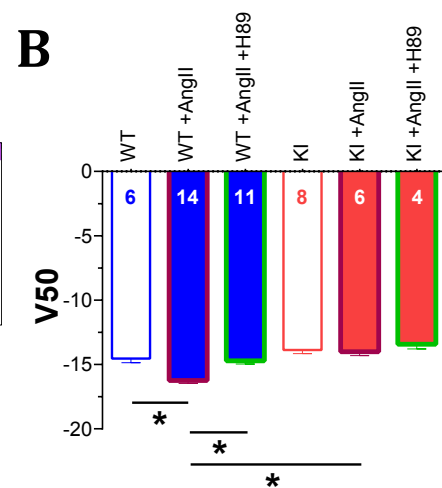

Figure 3.10. AngII increases open probability of calcium channel via oxidized PKA. $\mathrm{I}_{\mathrm{Ca}}$ activation curve (A), with relative conductance derived from maximal chord conductance and reversal potential (Erev) for each $\mathrm{I}-\mathrm{V}$, and peak $\mathrm{I}_{\mathrm{Ca}} /(\mathrm{Em}-\mathrm{Erev})$. The resulting conductance was normalized to the maximal chord conductance (usually between 10 and $20 \mathrm{mV}$ ). Data was fit to a standard Boltzmann equation. Panel B: Quantitative voltage data for half-activation (V50). AngII negatively-shifted the $\mathrm{I}_{\mathrm{Ca}}$ activation curve, and this was blocked with H89. However, this phenomenon was completely absent in KI. Quantitative data represented as mean \pm standard error. At least three independent mice were used per group; ANOVA was used for statistics; * means $\mathrm{P}<0.05$. 
$\mathrm{I}_{\mathrm{Ca}}$ steady-state activation gating was also investigated by measuring slope conductance at voltage-steps increasing from -40 to $80 \mathrm{mV}$, to further understand the mechanism of $\mathrm{I}_{\mathrm{Ca}}$ regulation by oxidized PKA. The slope conductance was then normalized to maximal conductance (G/Gmax, fig.3.10). Excitingly, in WT cardiomyocytes AngII significantly shifted the steady-state activation curve by about $2 \mathrm{mV}$ towards more negative potential. Therefore, reduced the voltage at half-maximal activation (V50) from $-14.54 \pm 0.32$ to $16.02 \pm 0.22 \mathrm{mV}(\mathrm{P}<0.05$, fig.3.10) in WT cardiomyocytes. This leftward shift in the activation curve was blocked by pre-incubating the cardiomyocytes with H89 (V50: $-14.68 \pm 0.24 \mathrm{mV}, \mathrm{P}<0.05$ vs. AngII). Interestingly, this AngII-dependent shift in the activation curve was completely absent in KI cardiomyocytes (V50 was $-13.88 \pm 0.27$ vs. $-14.01 \pm 0.31 \mathrm{mV}$, vehicle vs. AngII, P=N.S., fig.3.10), suggesting that oxidized PKA type I may be involved in $\mathrm{Ca}^{2+}$ channel gating. In accordance, H89 did not affect V50 in KI cardiomyocytes (V50 -13.43 $\pm 0.36 \mathrm{mV}$, fig.3.10).

\subsection{WT and KI have showed similar cardiac function in vivo}

In vitro contractile data showed no difference in WT and KI cardiomyocyte contractility at baseline. Therefore, the whole heart functional characterization was done by echocardiography, to investigate whether the contractility is similar at in vivo conditions.
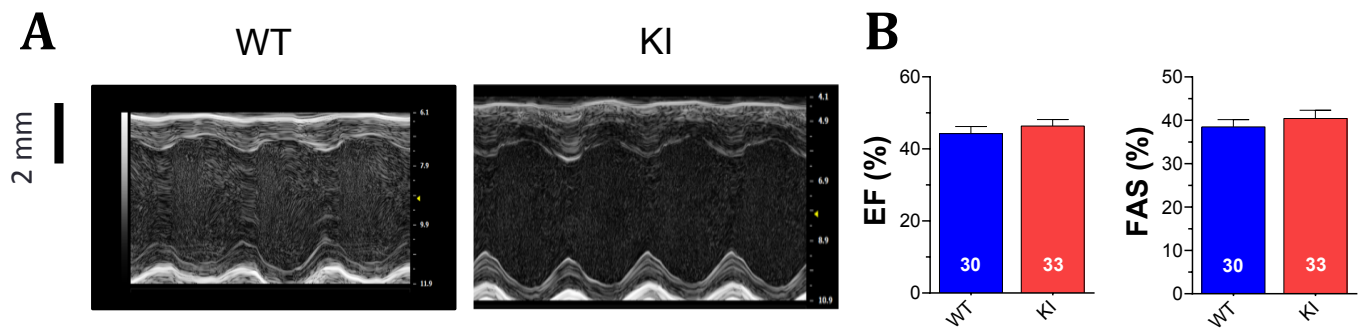

Figure 3.11. Redox-dead PKA mice have a similar cardiac contractile function like WT at baseline. Original recordings (A, left) and quantitative data represented as mean \pm standard error (B, right). M-mode echocardiography of WT and KI mice showed no difference in cardiac contraction (left). Quantitative data also showed no difference in EF and FAS of WT and KI mice (right). At least three independent mice were used per group.

Figure 3.11 shows original traces (M-mode) and quantitative analysis of ejection fraction (EF) and fractional area shortening (FAS). In accordance with the data from isolated ventricular cardiomyocytes, no differences in EF or FAS were ob- 
served. For WT vs. KI mice, EF were $44.25 \pm 1.96$ vs. $46.37 \pm 1.76 \%$; and FAS were $38.47 \pm 1.67$ vs. $40.41 \pm 1.91 \%$, P= N.S. Similarly, left ventricular internal diameters in diastole (LVIDd) or wall thicknesses were not different among the mice (table 3.1).

Table 3.1. Summary of echocardiographic parameters of WT and KI mice at baseline condition (mean \pm SEM).

\begin{tabular}{|l|c|c|}
\hline & WT & KI \\
\cline { 2 - 3 } Number & 30 & 33 \\
HR (bpm) & $407.667 \pm 11.176$ & $440.882 \pm 35.795$ \\
RR (min-1) & $119.267 \pm 8.031$ & $120.643 \pm 12.814$ \\
BT ( $\left.{ }^{\circ} \mathbf{C}\right)$ & $37.187 \pm 0.250$ & $37.350 \pm 0.314$ \\
BW (g) & $22.680 \pm 0.808$ & $21.594 \pm 0.699$ \\
FS (\%) & $22.983 \pm 1.228$ & $23.230 \pm 1.338$ \\
SV ( $\boldsymbol{\mu l}$ ) & $40.329 \pm 2.246$ & $38.225 \pm 1.982$ \\
CO (ml/min) & $16.389 \pm 0.989$ & $16.887 \pm 1.562$ \\
CI ( $\boldsymbol{\mu l} / \mathbf{m i n} / \mathbf{g})$ & $731.855 \pm 47.131$ & $787.962 \pm 76.519$ \\
LVIDd (mm) & $4.468 \pm 0.081$ & $4.330 \pm 0.069$ \\
LVIDs (mm) & $3.446 \pm 0.097$ & $3.327 \pm 0.085$ \\
Vol s ( $\boldsymbol{\mu l )}$ & $51.721 \pm 3.876$ & $44.476 \pm 2.438$ \\
Vol d ( $\boldsymbol{\mu l})$ & $92.050 \pm 4.985$ & $82.701 \pm 3.317$ \\
AWThd (mm) & $0.651 \pm 0.022$ & $0.618 \pm 0.026$ \\
PWThd (mm) & $0.550 \pm 0.022$ & $0.519 \pm 0.021$ \\
AWThs (mm) & $0.955 \pm 0.033$ & $0.918 \pm 0.033$ \\
PWThs (mm) & $0.786 \pm 0.023$ & $0.747 \pm 0.034$ \\
Ls (mm) & $7.035 \pm 0.116$ & $6.869 \pm 0.092$ \\
Ld (mm) & $7.799 \pm 0.145$ & $7.631 \pm 0.087$ \\
\hline
\end{tabular}

Analysis of basic surface electrocardiograms also revealed no differences in heart rate, intra-arterial, atrioventricular or intraventricular conduction or repolarization (table 3.2 ).

Table 3.2. Summary of electrocardiographic parameters of WT and KI mice at baseline condition (mean \pm SEM).

\begin{tabular}{|l|c|c|}
\hline & WT & KI \\
\cline { 2 - 3 } Number & 9 & 7 \\
RR Interval (s) & $0.124 \pm 0.0042$ & $0.123 \pm 0.0049$ \\
Heart Rate (BPM) & $488.933 \pm 17.5835$ & $491.186 \pm 18.7127$ \\
PR Interval (s) & $0.036 \pm 0.0013$ & $0.037 \pm 0.0015$ \\
P Duration (s) & $0.007 \pm 0.0005$ & $0.010 \pm 0.0017$ \\
QRS Interval (s) & $0.008 \pm 0.0004$ & $0.008 \pm 0.0005$ \\
QT Interval (s) & $0.019 \pm 0.0019$ & $0.019 \pm 0.0029$ \\
QTc (s) & $0.054 \pm 0.0055$ & $0.055 \pm 0.0084$ \\
JT Interval (s) & $0.011 \pm 0.0019$ & $0.011 \pm 0.0028$ \\
Tpeak Tend Interval (s) & $0.009 \pm 0.0018$ & $0.009 \pm 0.0029$ \\
P Amplitude (mV) & $0.138 \pm 0.0104$ & $0.101 \pm 0.0268$ \\
Q Amplitude (mV) & $-0.011 \pm 0.0083$ & $-0.020 \pm 0.0157$ \\
R Amplitude (mV) & $0.874 \pm 0.0863$ & $0.935 \pm 0.0694$ \\
S Amplitude (mV) & $-0.202 \pm 0.0351$ & $-0.246 \pm 0.0566$ \\
ST Height (mV) & $0.098 \pm 0.0187$ & $0.086 \pm 0.0101$ \\
T Amplitude (mV) & $0.182 \pm 0.0621$ & $0.160 \pm 0.0444$ \\
\hline
\end{tabular}

\subsection{AngII induces cytosolic oxidant production in vivo}

To test, whether AngII-, similar to the acute exposure in isolated ventricular cardiomyocytes, induced cytosolic ROS production, mouse ventricular cardiomyocytes were isolated two weeks after osmotic minipump mediated infusion of 


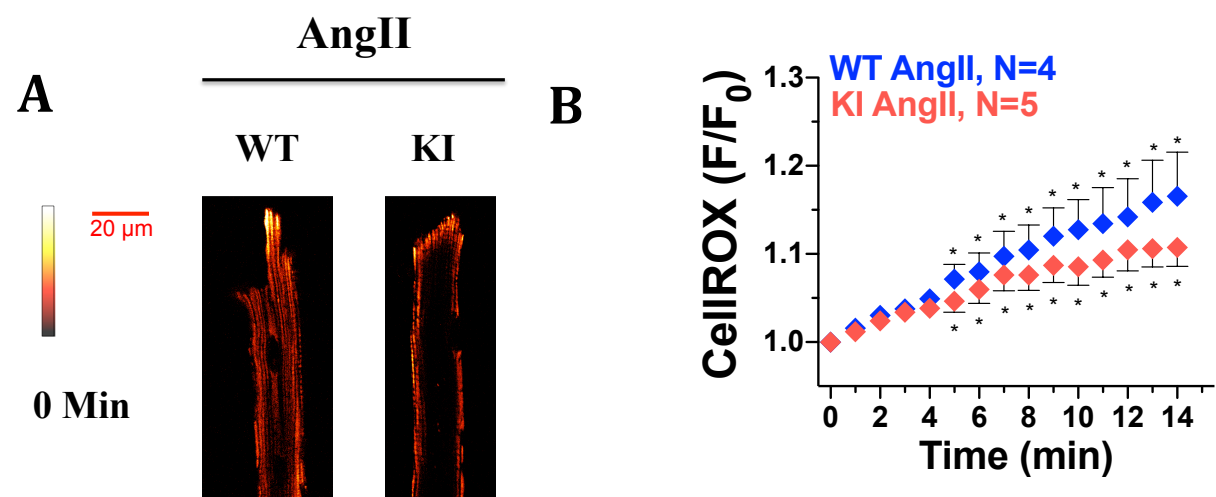

Figure 3.12. Chronic AngII infusion induces oxidant production both in WT and KI mice. Original recordings (A, left) and quantitative data represented as mean \pm standard error (B, right). Mice carried AngII containing OMP produced significantly higher oxidants as visualized by increased fluorescent signal from isolated ventricular cardiomyocytes loaded with cytosolic ROS indicator CellROX. For improved visualization, gray scale values were converted to color using the depicted calibration bar (A). Quantitative data also a showed similar pattern in WT and KI. At least two independent mice were used per group. ANOVA was used for statistics; * means $\mathrm{P}<0.05$.

AngII and loaded with CellROX $(2.5 \mu \mathrm{mol} / \mathrm{L}, 20 \mathrm{~min})$. Figure 3.12 shows original traces and quantitative data for CellROX fluorescence with a time-dependent increase indicative of steady-state ROS production. However, there was no significant difference between KI and WT mice upon chronic AngII infusion. At 14 min after the onset of measurement, CellROX fluorescence increased to $1.17 \pm 0.05$ vs. $1.11 \pm 0.02$ for WT vs. KI cardiomyocytes, P=N.S.

\subsection{AngII-induced LTCC phosphorylation via PKA is absent in}

\section{KI mice}

After two weeks of AngII infusion, mice hearts were analyzed to test whether chronic AngII infusion can cause PKA oxidation like in vitro experiments. Compared to vehicle infusion (control, CTRL), two weeks exposure to AngII significantly increased PKA RI dimer formation in WT hearts (fig.3.13). The ratios of PKA RI dimer to monomer were $0.17 \pm 0.07$ vs. $0.59 \pm 0.21(\mathrm{P}<0.05)$ for CTRL vs. AngII in WT mice. As expected this increased was completely absent in KI mice (fig.3.13). RI dimer/monomer ratios were $0.13 \pm 0.05$ vs. $0.10 \pm 0.04$ for CTRL vs. AngII ( $\mathrm{P}=\mathrm{N} . \mathrm{S}$.). Moreover, there was a strong trend toward reduced expression of PKA catalytic subunit (PKA C) upon long term AngII exposure (fig.3.13) with no 
difference between WT and KI mice. In WT, PKA C levels were $1.53 \pm 0.35$ vs. $1.22 \pm 0.34$ (CTRL vs. AngII, $\mathrm{P}=0.39$ ) and in KI levels were $1.51 \pm 0.21$ vs. $1.12 \pm 0.21$ (CTRL vs. AngII, $\mathrm{P}=0.31$ ).

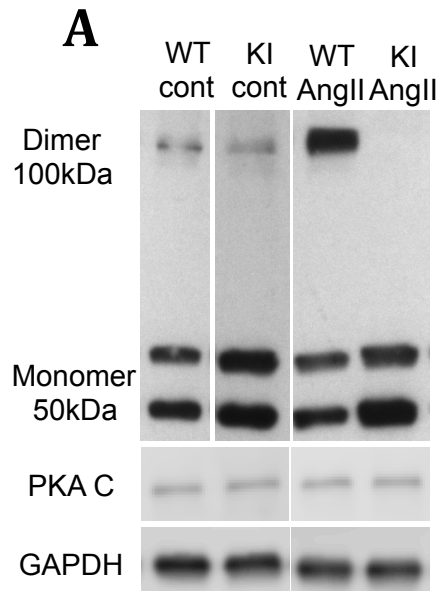

\section{B}
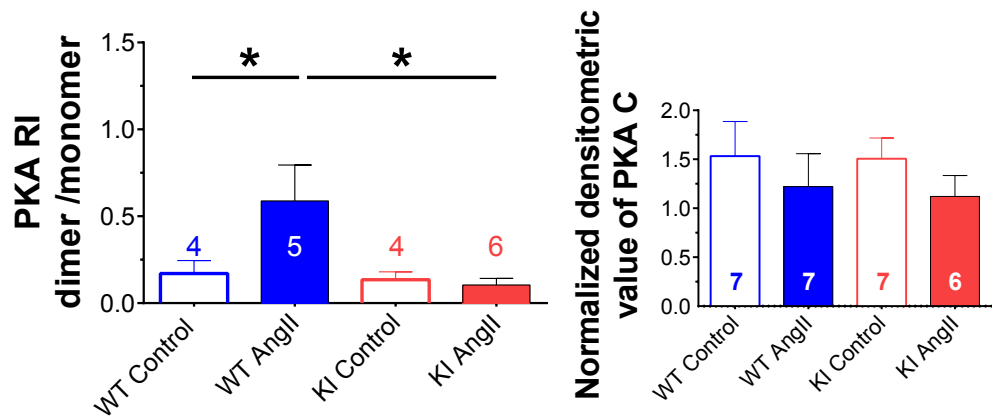

Figure 3.13. Chronic infusion of AngII induces PKA RI dimer formation. The amount of PKA RI dimer was increased in WT mice compared to KI upon two weeks of AngII infusion using osmotic minipumps (OMP) (A, left). Quantitative data represented as mean \pm standard error showing that amount of dimer were significantly higher in WT AngII group compared to control and KI group (B, right). However, the expression of the catalytic subunit of PKA decreased in both WT and KI upon AngII. At least three independent mice were used per group; ANOVA was used for statistics; * means $\mathrm{P}<0.05$.

LTCC phosphorylation was checked using western blot, to test whether PKAdependent LTCC phosphorylation (at Serine 1928) is also affected by two weeks AngII exposure. Compared to control, AngII exposure significantly increased pLTCC levels in WT mice (p-LTCC/LTCC was $2.28 \pm 0.27$ vs. $4.18 \pm 0.79$ vs. for CTRL vs. AngII, fig.3.14). Interestingly, this AngII-dependent increase in p-LTCC levels was completely absent in KI mice (fig.3.14). p-LTCC/LTCC levels were 2.91 \pm 0.32 vs. $2.41 \pm 0.34$ for CTRL vs. AngII in KI mice, P=N.S., fig.3.14).

Interestingly, there was a tendency towards reduced expression of LTCC upon AngII infusion in both WT and KI mice (fig.3.14). Since acute AngII exposure also affected SR $\mathrm{Ca}^{2+}$ content and SR $\mathrm{Ca}^{2+}$ leak, PKA-dependent phosphorylation of RyR2 and Phospholamban (PLB) was investigated in this chronic AngII infusion model. Figure 3.14 shows that RyR2 expression did not change upon AngII exposure neither in WT (CTRL vs. AngII, $1.12 \pm 0.09$ vs. $1.28 \pm 0.09$ ) nor KI mice (CTRL vs. AngII, $1.47 \pm 0.10$ vs. $1.43 \pm 0.10$; fig. 3.14 ). 


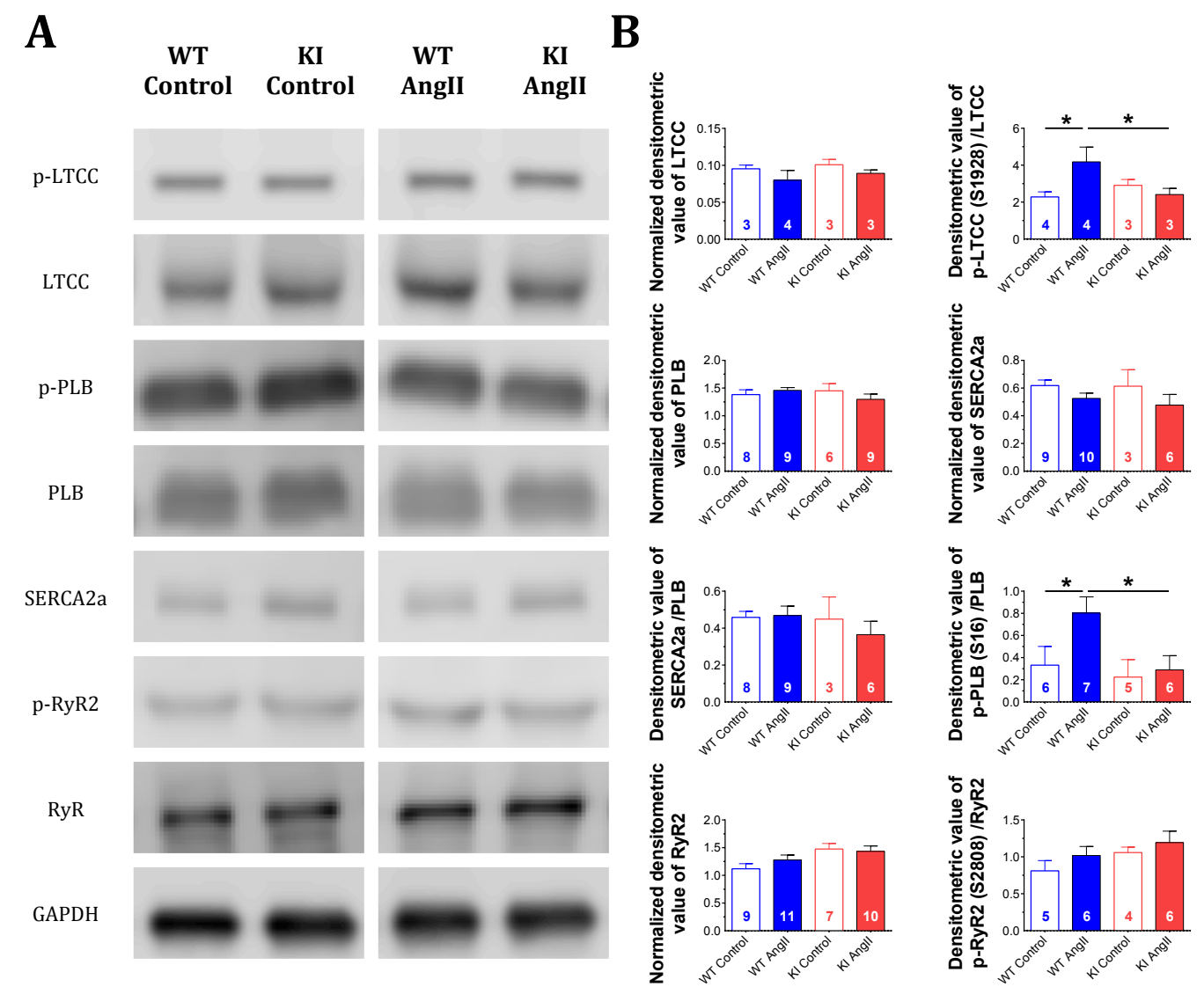

Figure 3.14. Chronic AngII-induced activation of PKA type I cause subsequent substrate phosphorylation. Oxidized PKA was considered as a phosphorylating agent for different substrates, like L-type $\mathrm{Ca}^{2+}$ Channel, Phospholamban, as shown in panel A. Although, the expressions were unchanged for LTCC, PLB, SERCA2a and RyR2; the PKA phosphorylation sites were significantly more phosphorylated in WT compared to control. KI mice could not show this phenomenon, indicating oxidized PKA is involved. RyR2 also did not show this oxidative PKAdependent phosphorylation, meaning it is not a site of oxidative modification (B). Quantitative data represented as mean \pm standard error. At least three independent mice were used per group; ANOVA was used for statistics; * means $\mathrm{P}<0.05$.

Moreover, although there was a tendency of RyR2 towards increased phosphorylation at S2808 upon AngII exposure, this increase was not significant for WT and KI mice (WT, CTRL vs. AngII, $0.81 \pm 0.14$ vs. $1.02 \pm 0.12$; KI, CTRL vs. AngII, $1.06 \pm 0.08$ vs. $1.19 \pm 0.16$; fig.3.14). In contrast, AngII exposure significantly increased PKA-dependent PLB phosphorylation at S16 in WT animals (CTRL vs. AngII, $0.33 \pm 0.17$ vs. $0.80 \pm 0.15, \mathrm{P}<0.05$, fig.3.14). This increase, however, was completely absent in KI mice (CTRL vs. AngII $0.22 \pm 0.16$ vs. $0.29 \pm 0.13$ ). Moreover, global SERCA2a and PLB expression and SERCA2a/PLB ratios were not different between these two mice upon chronic AngII (fig.3.14).

In order to test, whether other important serine/threonine kinases, which can 
regulate ion channels and transporters, are activated by two weeks AngII exposure, CaMKII expression and auto-phosphorylation (at threonine 287) of (indicative of CaMKII activity) were measured. Figure 3.15 show that AngII significantly increased CaMKII expression with no difference between WT and KI mice (fig.3.15). In WT animal compared to control, AngII increased CaMKII expression from $1.01 \pm 0.06$ to $1.33 \pm 0.10, \mathrm{P}<0.05$, fig.3.15). In KI mice CaMKII expression was $1.06 \pm 0.06$ vs. $1.44 \pm 0.07$, for CTRL vs. AngII, $\mathrm{P}<0.05$, fig.3.15). Analysis of pCaMKII/CaMKII level, however, revealed only a trend towards more CaMKII auto-phosphorylation with AngII exposure and this trend was similar for WT vs. KI mice (fig.3.15).
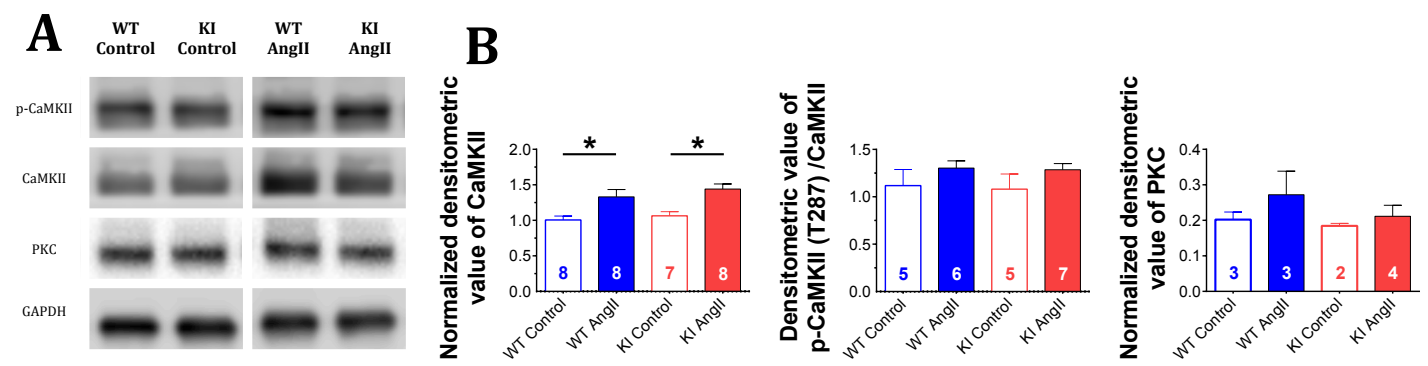

Figure 3.15. Effects of AngII on CaMKII and PKC expression. CaMKII and phospho-CaMKII levels were get increased in both WT and KI upon AngII (A: Original data, B: quantitative data represented as mean \pm standard error). However, the level of PKC expression was increased a bit upon AngII in both WT and KI (B). At least three independent mice were used per group; ANOVA was used for statistics; * means $\mathrm{P}<0.05$.

\subsection{AngII induces contractile dysfunction in mice lacking oxi- dative activation of PKA}

AngII infused mice were subjected to echocardiography to assess in vivo cardiac functions, to test whether AngII-induced PKA type I-dependent regulation of LTCC is essential for contractile function. Figure 3.16 shows original traces (Mmode) and mean data for echocardiography in mice after two weeks exposure to AngII. Similar to the acute exposure of AngII in ventricular cardiomyocytes, two weeks AngII infusion neither alters ejection fraction nor FAS in WT mice (fig.3.16, EF was $50.99 \pm 2.28$ vs. $48.86 \pm 2.39$ for CTRL vs. AngII, P=N.S.; FAS was $44.76 \pm 2.40$ vs. $43.90 \pm 2.16$ for CTRL vs. AngII, P=N.S.). In sharp contrast, FAS was significantly reduced in KI mice upon two weeks of AngII infusion (fig.3.16; FAS was $43.38 \pm 3.14$ vs. $36.22 \pm 2.33$, for CTRL vs. AngII, $\mathrm{P}<0.05$ ). Thus, the PKA- 
dependent LTCC phosphorylation may indeed be vital for contractile function, also upon long term AngII exposure.

A

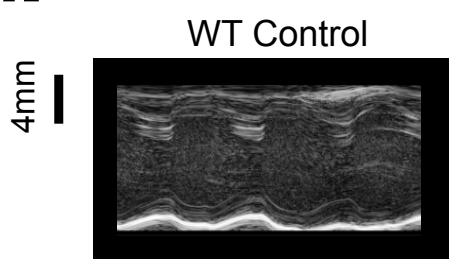

WT Angll

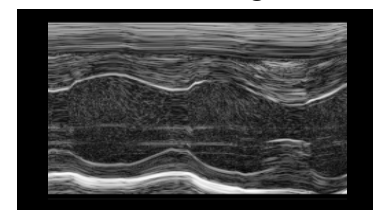

B

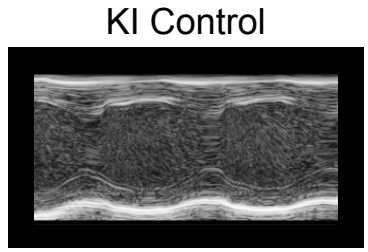

KI Angll
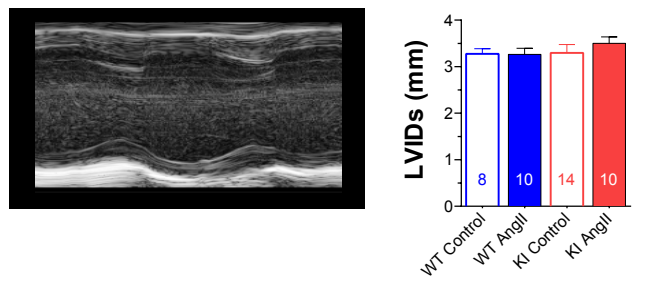
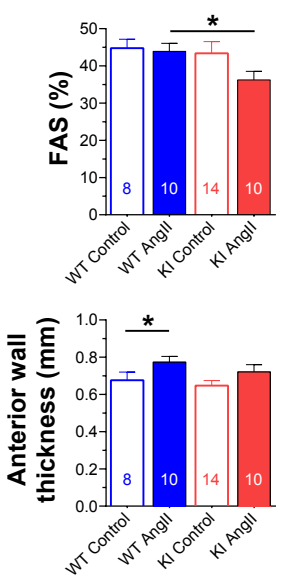

\section{C}
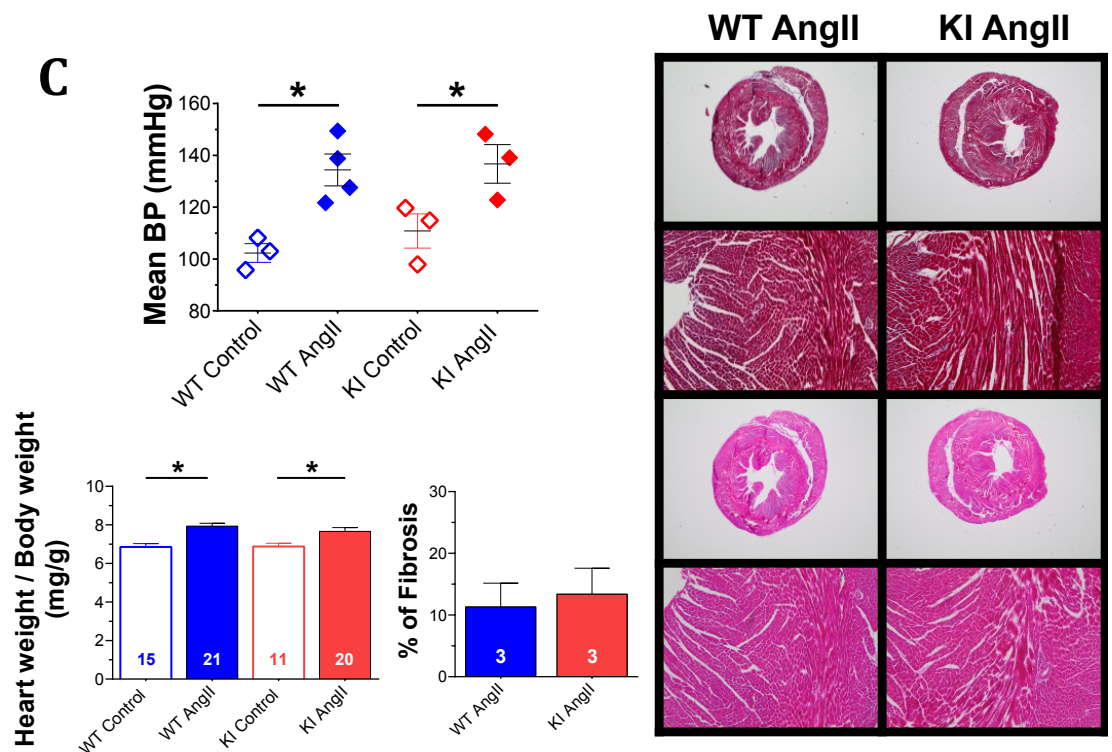

Figure 3.16. Chronic AngII infusion alters cardiac function. In vivo echocardiography reveled that KI had reduced cardiac function compared to WT and control (A). Quantitative data represented as meantstandard error showing less ejection fraction and fractional area shortening in KI compared to WT littermates (B). Upon two weeks of AngII infusion, both WT and $\mathrm{KI}$ had increased blood pressure compared to the control (C). MTC and Hematoxylin staining showed that similar morphology of whole heart sections upon AngII infusion in both groups of mice. Although WT showed more hypertrophy compared to KI as reveled by heart weight to body weight ratio $(C)$, both the mice had a similar level of fibrosis. At least three independent mice were used per group. ANOVA was used for statistics; * means $\mathrm{P}<0.05$.

Interestingly, AngII exposure also resulted in left ventricular hypertrophy with no difference between WT and KI mice. Compared to control, there was a strong trend for larger anterior wall thickness in both WT and KI animals $[0.68 \pm 0.04$ vs. $0.77 \pm 0.03 \mathrm{~mm}, \mathrm{P}<0.05$, for WT and $0.65 \pm 0.03$ vs. $0.72 \pm 0.04 \mathrm{~mm}, \mathrm{P}=0.12$, for $\mathrm{KI}$ ). There was also a tendency towards greater left ventricular inner diameter in KI 
mice upon AngII (WT vs. KI; $3.26 \pm 0.13$ vs. $3.50 \pm 0.14 \mathrm{~mm}$ ), which was higher in KI considered to WT mice.

The cause of left ventricular hypertrophy may be compensatory to increased arterial blood pressure in the presence of AngII. Figure 3.16 shows mean arterial blood pressure measured by tail-cuff measurements. AngII exposure significantly increased mean arterial blood pressure in both WT and KI animals. In WT mice, mean arterial blood pressure were $102.3 \pm 3.59$ vs. $140.0 \pm 7.43 \mathrm{mmHg}$ for CTRL vs. AngII, $\mathrm{P}<0.05$, fig.3.16). Similarly, in KI mice mean blood pressure were 110.8 \pm 6.61 vs. $136.7 \pm 7.43 \mathrm{mmHg}$ for CTRL vs. AngII, $\mathrm{P}<0.05$, fig.3.16).

Table 3.3. Summary of echocardiographic parameters of WT and KI mice after two weeks of saline or AngII infusion (mean \pm SEM).

\begin{tabular}{|c|c|c|c|c|}
\hline & WT Control & WT AngII & KI Control & KI AngII \\
\hline Number & 8 & 14 & 10 & 10 \\
\hline HR (bpm) & $430.000 \pm 21.707$ & $431.286 \pm 12.944$ & $445.300 \pm 12.957$ & $424.100 \pm 14.164$ \\
\hline RR (min-1) & $108.857 \pm 16.338$ & $123.417 \pm 8.800$ & $115.125 \pm 16.138$ & $124.500 \pm 6.544$ \\
\hline BT $\left({ }^{\circ} \mathrm{C}\right)$ & $37.250 \pm 0.290$ & $37.114 \pm 0.216$ & $37.240 \pm 0.327$ & $36.890 \pm 0.261$ \\
\hline BW (g) & $26.063 \pm 1.099$ & $26.457 \pm 0.934$ & $26.460 \pm 1.153$ & $28.600 \pm 1.105$ \\
\hline FS (\%) & $25.632 \pm 1.117$ & $25.417 \pm 1.652$ & $24.716 \pm 2.234$ & $21.259 \pm 1.634$ \\
\hline $\operatorname{SV}(\mu \mathrm{l})$ & $45.509 \pm 4.636$ & $43.223 \pm 2.410$ & $42.938 \pm 2.611$ & $39.819 \pm 2.462$ \\
\hline $\mathrm{CO}(\mathrm{ml} / \mathrm{min})$ & $19.798 \pm 2.517$ & $18.633 \pm 1.137$ & $19.207 \pm 1.411$ & $17.129 \pm 1.463$ \\
\hline $\mathrm{CI}(\mu \mathrm{l} / \mathrm{min} / \mathrm{g})$ & $747.175 \pm 69.978$ & $711.007 \pm 43.876$ & $729.862 \pm 53.645$ & $596.835 \pm 45.232$ \\
\hline LVIDd (mm) & $4.407 \pm 0.150$ & $4.364 \pm 0.117$ & $4.352 \pm 0.146$ & $4.433 \pm 0.103$ \\
\hline Vol s $(\mu \mathrm{l})$ & $43.752 \pm 4.303$ & $47.017 \pm 4.235$ & $46.404 \pm 5.500$ & $52.092 \pm 5.701$ \\
\hline Vol d $(\mu \mathrm{l})$ & $89.261 \pm 7.575$ & $90.240 \pm 5.414$ & $89.342 \pm 7.049$ & $91.911 \pm 6.660$ \\
\hline PWThd (mm) & $0.538 \pm 0.040$ & $0.608 \pm 0.029$ & $0.603 \pm 0.020$ & $0.636 \pm 0.042$ \\
\hline AWThs (mm) & $0.985 \pm 0.065$ & $1.068 \pm 0.038$ & $0.990 \pm 0.025$ & $1.012 \pm 0.043$ \\
\hline PWThs (mm) & $0.762 \pm 0.032$ & $0.861 \pm 0.038$ & $0.758 \pm 0.033$ & $0.825 \pm 0.048$ \\
\hline Ls (mm) & $7.089 \pm 0.174$ & $7.223 \pm 0.166$ & $7.059 \pm 0.175$ & $7.106 \pm 0.300$ \\
\hline Ld (mm) & $8.000 \pm 0.192$ & $8.092 \pm 0.130$ & $7.908 \pm 0.183$ & $8.112 \pm 0.230$ \\
\hline
\end{tabular}

Like the echo data, AngII infusion significantly increased heart weight (normalized to body weight) with no significant difference between WT and KI mice. Immunohistochemistry was performed to investigate whether altered cardiac function is due to their hypertrophic or fibrotic responses upon AngII. Masson's trichrome and H/E staining showed that both WT and KI animals had similar morphology after AngII infusion. Quantitative data also revealed that both WT and KI mice had a similar level of fibrosis upon AngII (WT vs. KI; $11.32 \pm 3.84$ vs. $13.38 \pm 4.21 \%$ Fibrosis).

\subsection{AngII induces calcium channel dysregulation in mice lack- ing oxidative activation of PKA}

In order to test, whether $\mathrm{Ca}^{2+}$ channel dysregulation indeed underlies the contractile dysfunction observed in KI mice upon AngII exposure, $\mathrm{Ca}^{2+}$ channel activities was measured using the whole cell patch clamp technique and compared 
with the $\mathrm{I}_{\mathrm{Ca}}$ of isolated ventricular cardiomyocytes from mice chronically infused with AngII. Figure 3.17 show original traces and quantitative data for $\mathrm{I}_{\mathrm{Ca}}$.

A
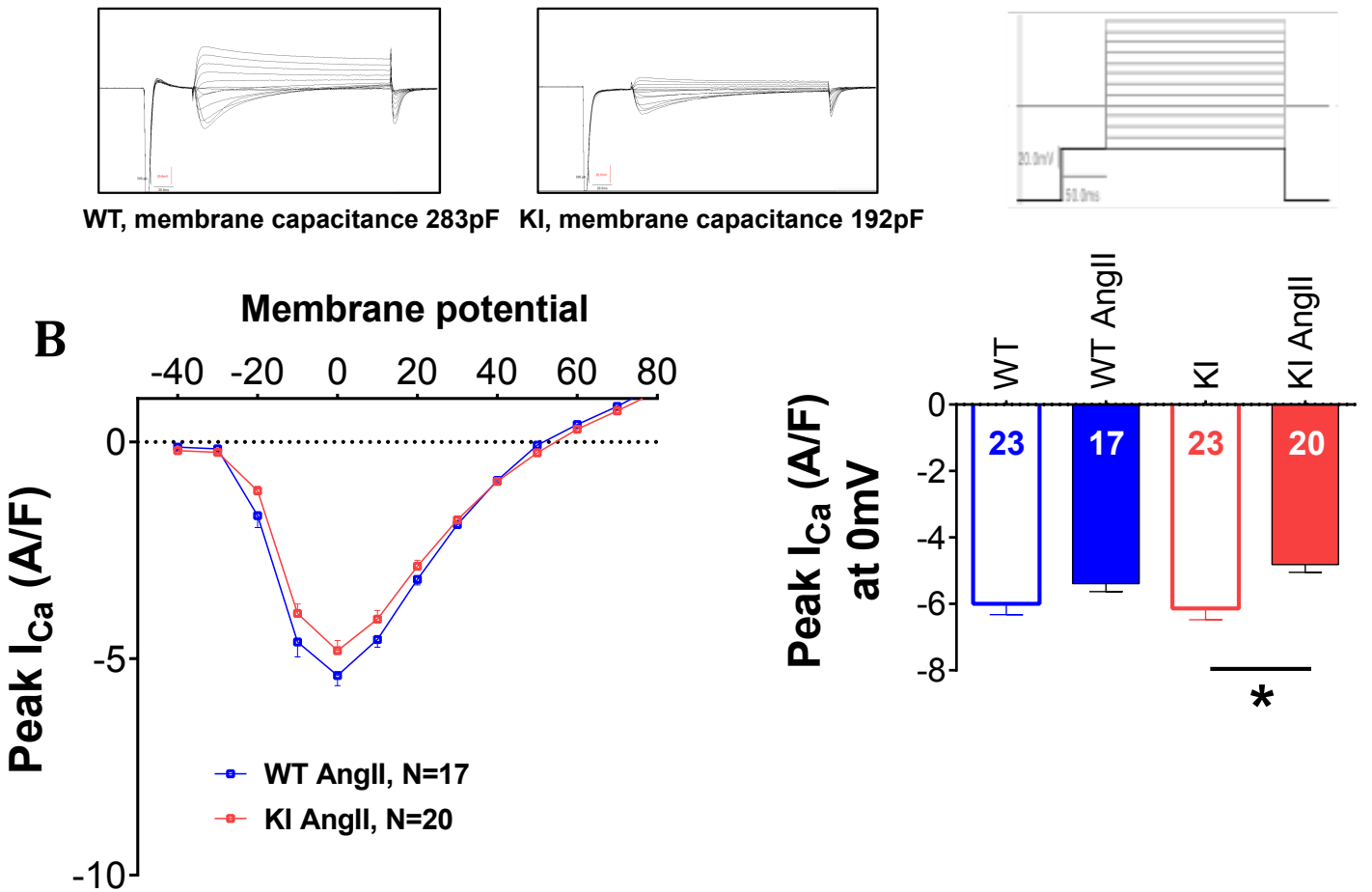

Figure 3.17. Oxidative PKA activation maintains $\mathrm{Ca}^{2+}$ channel function under chronic AngII infusion. Whole cell patch clamp revealed peak $\mathrm{Ca}^{2+}$ current amplitude was reduced in $\mathrm{KI}$ after two weeks of AngII exposure (A). In WT mice, peak $\mathrm{I}_{\mathrm{Ca}}$ was not altered. A) Original traces (left panel) and IV protocol (right panel) for current-voltage relationship B) Quantitative data represented as mean \pm standard error for IV curve and peak $\mathrm{I}_{\mathrm{Ca}}$ at $0 \mathrm{mV}$. At least three independent mice were used per group. ANOVA was used for statistics; * means $\mathrm{P}<0.05$.

Analysis of current-voltage relationship revealed no change in current amplitude in WT mice upon two weeks of AngII exposure (fig.3.17). However, exposure to AngII in KI mice resulted in a significantly reduced peak $\mathrm{Ca}^{2+}$ current amplitude. Since expression of LTCC was unchanged in both mice (see above), this reduction in current amplitude further confirms the altered regulation of LTCC, as revealed by western blot. Mean data for $\mathrm{I}_{\mathrm{Ca}}$ at $0 \mathrm{mV}$ were $-5.99 \pm 0.33$ vs. $-5.39 \pm 0.23$ (P=N.S.) A/F, for CTRL vs. AngII in WT, and $-6.14 \pm 0.34$ vs. $-4.82 \pm 0.23 \mathrm{~A} / \mathrm{F}$ $(\mathrm{P}<0.05)$ for CTRL vs. AngII in KI (fig.3.17).

Intracellular $\mathrm{Ca}^{2+}$ handling was investigated in isolated ventricular cardiomyocytes loaded with the $\mathrm{Ca}^{2+}$ indicator Fura-2 (10 $\left.\mu \mathrm{mol} / \mathrm{L}, 20 \mathrm{~min}\right)$, to understand further the mechanisms, which leads to contractile dysfunction in KI mice after two weeks of AngII exposure. In accordance with the echocardiographic data 
recorded in vivo, there was no difference in $\mathrm{Ca}^{2+}$ transient amplitude upon two weeks AngII exposure to WT cardiomyocytes (fig.3.18). In sharp contrast, AngII exposure dramatically reduced $\mathrm{Ca}^{2+}$ transient amplitude in $\mathrm{KI}$ cardiomyocytes (fig.3.18). At $0.5 \mathrm{~Hz}$, mean $\mathrm{Ca}^{2+}$ transient amplitudes were $0.20 \pm 0.03$ vs. $0.24 \pm 0.03$ for CTRL vs. AngII (P=N.S.) in WT, and $0.21 \pm 0.03$ vs. $0.12 \pm 0.01$ for CTRL vs. AngII $(\mathrm{P}<0.05)$ in KI cardiomyocytes (fig.3.18).

Despite increases in serine 16 phosphorylation of PLB (see above), there was no significant difference in $\mathrm{Ca}^{2+}$ transient decay kinetics after two weeks of AngII exposure both in WT and KI cardiomyocytes (fig.3.18). Nevertheless, in WT cardiomyocytes there was a trend toward faster $\mathrm{Ca}^{2+}$ transient decay after AngII exposure. At $0.5 \mathrm{~Hz}$, the time constant for $\mathrm{Ca}^{2+}$ transient decay tau decreased with AngII from $0.38 \pm 0.10$ to $0.35 \pm 0.07 \mathrm{sec}(\mathrm{P}=0.80)$ in WT cardiomyocytes. In KI cardiomyocytes, however, tau was unchanged (for CTRL vs. AngII $0.47 \pm 0.06$ vs. $0.437 \pm 0.07$ sec, $P=$ N.S., fig.3.18).

Interestingly, despite unchanged $\mathrm{Ca}^{2+}$ transient amplitude, a dramatically reduced SR $\mathrm{Ca}^{2+}$ content in WT cardiomyocytes was found by caffeine exposure upon chronic AngII exposure. In WT, AngII exposure significantly reduced caffeine-transient amplitude from $0.77 \pm 0.16$ to $0.42 \pm 0.07$, $\mathrm{P}<0.05$, fig.3.18). In $\mathrm{KI}$ cardiomyocytes, however, as shown above, caffeine-transient amplitude was already reduced at baseline (to $0.46 \pm 0.04, \mathrm{P}<0.05$ vs. WT, fig.3.18). In addition to this reduction, two weeks AngII-exposure was further reduced caffeine-transient amplitude in KI cardiomyocytes to $0.32 \pm 0.03$ ( $\mathrm{P}<0.05$ vs. KI CTRL and WT AngII, fig.3.18).

Thus, the unchanged $\mathrm{Ca}^{2+}$ transient amplitude in WT cardiomyocytes, despite an AngII-dependent reduction in SR $\mathrm{Ca}^{2+}$ content, may be due to increasing fractional $\mathrm{Ca}^{2+}$ release from the SR $\left(\mathrm{Ca}^{2+}\right.$ transient amplitude normalized to $\mathrm{Ca}^{2+}$ current). AngII exposure strongly increased fractional release from 2.289 to $3.49 \pm 0.72 \%, \mathrm{P}<0.05$, fig.3.18). In $\mathrm{KI}$, however, fractional $\mathrm{Ca}^{2+}$ release was not able to compensate (for CTRL vs. AngII $1.85 \pm 0.32$ vs. $1.71 \pm 0.18 \%, \mathrm{P}=\mathrm{N} . \mathrm{S}$., 
fig.3.18). Therefore, reduced $\mathrm{Ca}^{2+}$ influx through LTCC resulting in dramatically impaired $\mathrm{Ca}^{2+}$ transient amplitude leading to disturbed contractile function.
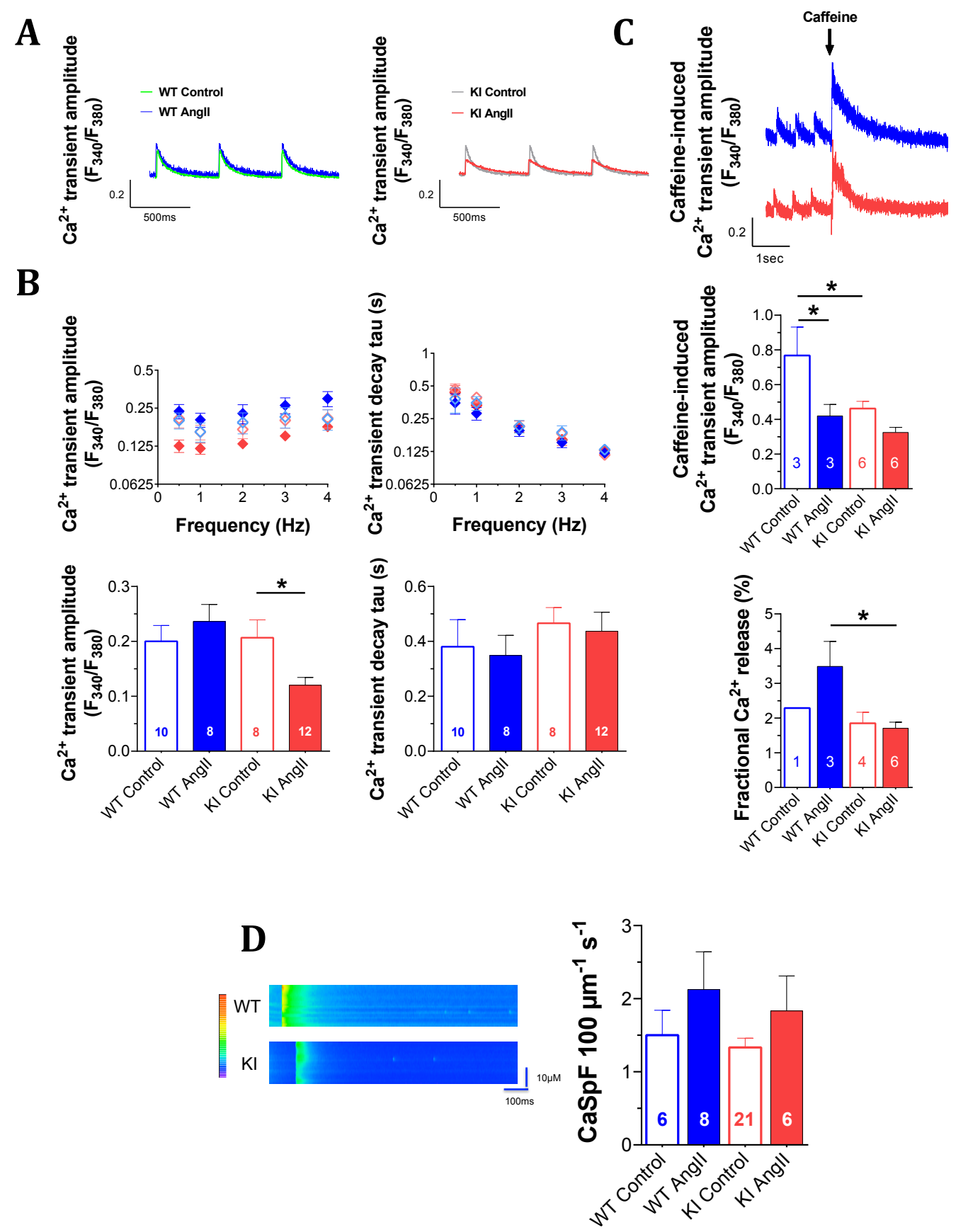

Figure 3.18. Oxidative PKA activation modulates $\mathrm{Ca}^{2+}$ transient but not $\mathrm{Ca}^{2+}$ spark upon chronic AngII infusion. Top panel showed original contractile traces of Fura-2-loaded cardiomyocytes stimulated at $0.5 \mathrm{~Hz}$ from two weeks AngII infused mice (A). Upon increasing the frequency, both WT and KI mice increased $\mathrm{Ca}^{2+}$ transient amplitude. However, quantitative data represented as mean \pm standard error showing that at $0.5 \mathrm{~Hz}$ the amplitude was less and the $\mathrm{Ca}^{2+}$ reuptake was slower in KI mice exposing to AngII (B). Representative caffeine-induced $\mathrm{Ca}^{2+}$ transients showed both WT and KI had reduced SR calcium content upon AngII, but KI had significantly lower $\mathrm{Ca}^{2+}$ content compared to WT and control (C, bottom). Fractional release data also showed that WT cardiomyocytes increased fractional release upon AngII but KI could not. Interestingly, both the WT and KI showed increased spark production upon AngII (D). At least three independent mice were used per group, ANOVA and t-test were used for statistics, * means $\mathrm{P}<0.05$. 
Moreover, diastolic SR $\mathrm{Ca}^{2+}$ leak was investigated to understand the mechanisms of reduced SR $\mathrm{Ca}^{2+}$ content further by measuring spontaneous elementary $\mathrm{Ca}^{2+}$ release events (i.e. $\mathrm{Ca}^{2+}$ sparks) using confocal microscopy. Figure 3.18 show that AngII strongly increased $\mathrm{Ca}^{2+}$ spark frequency with no difference between WT and KI cardiomyocytes. AngII increased $\mathrm{Ca}^{2+}$ spark frequency from $1.50 \pm 0.34$ to $2.13 \pm 0.521 / 100 \mu \mathrm{m}^{-1} \mathrm{~s}^{-1}(\mathrm{P}=0.22)$ in $\mathrm{WT}$, and from $1.33 \pm 0.13$ to $1.83 \pm 0.481 / 100$ $\mu \mathrm{m}^{-1} \mathrm{~s}^{-1}(\mathrm{P}=0.25)$ in KI cardiomyocytes (fig.3.18). Since PKA-dependent phosphorylation of RyR2 was unchanged, it is highly unlikely that altered PKA activity is responsible for this AngII-mediated effect.

\subsection{Chronic AngII exposure does not increase the propensity}

\section{for ventricular arrhythmias in mice}

Electrocardiogram (ECG) and programmed electrical stimulations were performed on chronically AngII infused WT and KI mice, to test whether AngIIdependent changes in $\mathrm{Ca}^{2+}$ handling would translate into increased propensity for arrhythmias. Two weeks exposure to AngII did not influence surface ECG parameters for conduction and repolarization of mice as visualized by original ECG recordings (fig.3.19) and also in the mean data (table 3.4). Burst stimulation of the right ventricular apex did not induce ventricular arrhythmias in either WT or KI mice after two weeks of AngII exposure (6 mice per group), which was also revealed by Fishers exact test 0 of 6 in WT vs. 1 of 6 in KI, P=1.0 (fig.3.19).

A

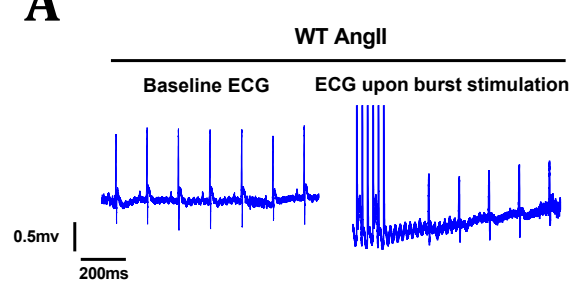

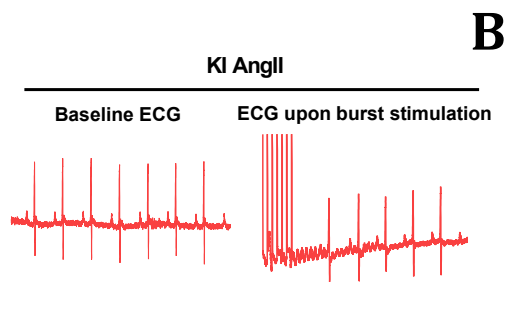

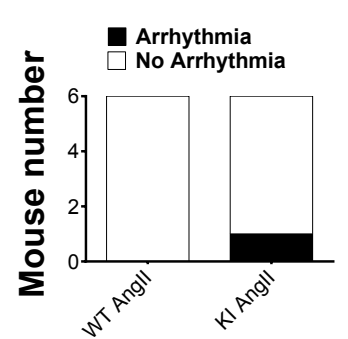

Figure 3.19. Chronic infusion of AngII to WT and KI mice do not induce arrhythmia. Original ECG traces showed similar ECG parameters in WT and KI mice after two weeks of AngII exposure (A, left). Moreover, in vivo programmed electrical burst stimulation could not induce a significant number of arrhythmias in any group of mice (B). At least three independent mice were used per group. 
Table 3.4. Summary of electrocardiographic parameters of WT and KI mice after two weeks of saline or AngII infusion (mean \pm SEM).

\begin{tabular}{|l|c|c|c|c|}
\hline & WT Control & WT AngII & KI Control & KI AngII \\
\cline { 2 - 5 } Number & 2 & 2 & 3 & 2 \\
RR Interval (s) & $0.122 \pm 0.0147$ & $0.125 \pm 0.0175$ & $0.132 \pm 0.0077$ & $0.114 \pm 0.0016$ \\
Heart Rate (BPM) & $499.100 \pm 60.3001$ & $488.700 \pm 68.3000$ & $456.733 \pm 28.2006$ & $525.850 \pm 7.1499$ \\
PR Interval (s) & $0.041 \pm 0.0049$ & $0.034 \pm 0.0001$ & $0.038 \pm 0.0032$ & $0.033 \pm 0.0004$ \\
P Duration (s) & $0.010 \pm 0.0011$ & $0.010 \pm 0.0008$ & $0.008 \pm 0.0008$ & $0.009 \pm 0.0014$ \\
QRS Interval (s) & $0.011 \pm 0.0039$ & $0.009 \pm 0.0003$ & $0.010 \pm 0.0015$ & $0.009 \pm 0.0001$ \\
QT Interval (s) & $0.019 \pm 0.0024$ & $0.025 \pm 0.0003$ & $0.020 \pm 0.0022$ & $0.018 \pm 0.0010$ \\
QTc (s) & $0.055 \pm 0.00347$ & $0.069 \pm 0.0057$ & $0.055 \pm 0.0079$ & $0.053 \pm 0.0033$ \\
JT Interval (s) & $0.008 \pm 0.0015$ & $0.016 \pm 0.0001$ & $0.009 \pm 0.0008$ & $0.009 \pm 0.0009$ \\
Tpeak Tend Interval (s) & $0.006 \pm 0.001848$ & $0.013 \pm 0.0008$ & $0.007 \pm 0.0004$ & $0.007 \pm 0.0008$ \\
P Amplitude (mV) & $0.114 \pm 0.0398$ & $0.163 \pm 0.0350$ & $0.139 \pm 0.0068$ & $0.189 \pm 0.0311$ \\
Q Amplitude (mV) & $-0.063 \pm 0.0277$ & $-0.033 \pm 0.0084$ & $0.000 \pm 0.0088$ & $-0.021 \pm 0.0181$ \\
R Amplitude (mV) & $1.003 \pm 0.1748$ & $1.103 \pm 0.1020$ & $1.097 \pm 0.2003$ & $0.942 \pm 0.1657$ \\
S Amplitude (mV) & $-0.349 \pm 0.0303$ & $-0.298 \pm 0.1295$ & $-0.393 \pm 0.0523$ & $-0.495 \pm 0.1518$ \\
T Amplitude (mV) & $0.085 \pm 0.0495$ & $0.201 \pm 0.0209$ & $0.139 \pm 0.0444$ & $0.126 \pm 0.0084$ \\
\hline
\end{tabular}

\subsection{Oxidized PKA and heart failure}

ROS production is well documented in AngII-mediated hypertrophic conditions and heart failure situations (Heymes et al., 2003). To investigate the involvement of redox-active PKA in heart failure conditions, I have used transverse aortic constriction model (TAC) in redox-dead PKA mice as well as their WT littermates. In this procedure constriction of aorta increases afterload-mediated ventricular wall stress and ultimately hypertrophy results. However, this ventricular hypertrophy along with numerous other structural adaptations may lead to ventricular dysfunction and, eventually, heart failure.

\subsection{TAC surgery induces cytosolic oxidant production}

In order to test, if chronic pressure overload by transverse aortic constriction may result in increased generation of reactive oxygen species, isolated ventricular cardiomyocytes from TAC-operated mice were investigated in the presence of CellROX. Upon 6 weeks of TAC, isolated cardiomyocytes from both KI and WT mice showed increased CellROX fluorescence. Similar to the data obtained in mice having CTRL OMP, sham-surgery did not affect ROS production (fig.3.20). CellROX fluorescence changed only minimally in WT and KI cardiomyocytes after sham surgery (fig.3.20). In contrast, after TAC, there was a time-dependent increase in CellROX fluorescence in both WT and KI cardiomyocytes, which is an indicative of increased ROS production (fig.3.20). At 14 min of measurement, CellROX fluorescence was $1.05 \pm 0.04$ vs. $1.15 \pm 0.05(\mathrm{P}<0.05)$ for Sham vs. TAC in WT; and $1.11 \pm 0.06$ vs. $1.13 \pm 0.02(\mathrm{P}<0.05)$ for Sham vs. TAC in KI (fig.3.20). No 
difference in CellROX fluorescence between WT and KI cardiomyocytes were found after TAC.

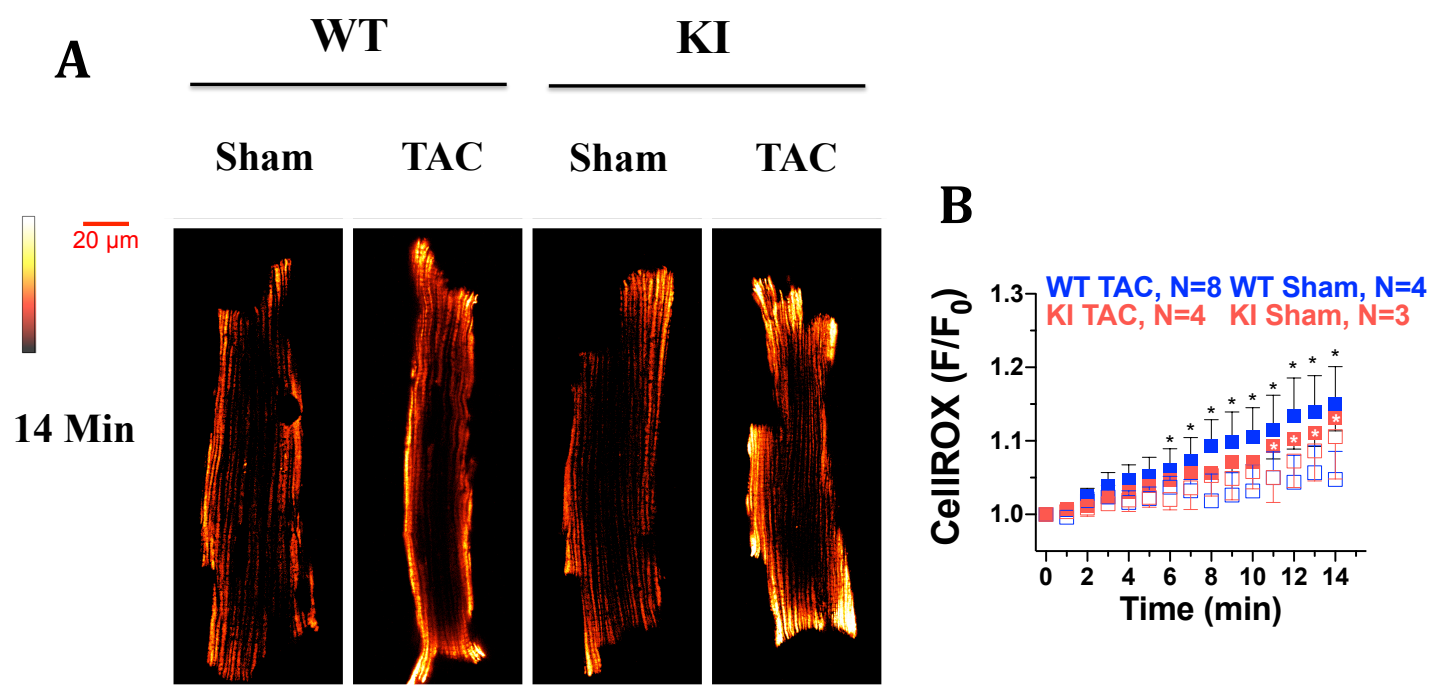

Figure 3.20. Pressure overload induces ROS production in cardiomyocytes. Original recordings (A, left) and quantitative data represented as mean \pm standard error (B, right). Isolated ventricular cardiomyocytes from TAC- or sham-operated WT and KI mice loaded with cytosolic ROS indicator CellROX, were monitored for 14 minutes (A). For improved visualization, grayscale values were converted to color using the depicted calibration bar (A). Both WT and KI cardiomyocytes produced oxidants after TAC operation. At $7 \mathrm{~min}$, CellROX fluorescence $\left(\mathrm{F} / \mathrm{F}_{0}\right)$ intensity, were increased significantly in WT cardiomyocytes and also showed a similar tendency in $\mathrm{KI}$ cardiomyocytes at $11 \mathrm{~min}$ (B). At least three independent mice were used per group, ANOVA was used for statistics; * means $\mathrm{P}<0.05$ vs. 0 min data.

\subsection{Type I PKA increases substrate phosphorylation upon pressure overload}

Pressure overload stress could initiate oxidative PKA activation pathway. In order to test, if increased ROS upon TAC would result in oxidative PKA activation, PKA RI dimer formation was assessed. Figure 3.20 show that RI dimer/monomer ratio increased significantly in WT mice after TAC (Sham vs. TAC, 0.319 \pm 0.134 vs. 1.156 \pm 0.413$)$. In sharp contrast, similar to data obtained upon AngII exposure, TAC could not increase PKA RI oxidation in KI mice (fig.3.21) (Sham vs. TAC, $0.02 \pm 0.01$ vs. $0.14 \pm 0.04$ ).

In order to test, if increased oxidized PKA upon TAC would result in increased phosphorylation of LTCC, PKA-dependent phosphorylation was analyzed by western blotting. Figure 3.22 shows that TAC surgery led to increasing in $\alpha 1_{c}$ phosphorylation at serine 1928 in WT mice (p-LTCC/LTCC levels were Sham vs. TAC, $1.28 \pm 0.04$ vs. $3.21 \pm 0.68, \mathrm{P}<0.05$, fig.3.22). In accordance with abolished ox- 
idative PKA RI dimer, no significant increase in p-LTCC/LTCC levels was found in KI mice after TAC (Sham vs. TAC, $1.348 \pm 0.245$ vs. $2.115 \pm 0.029$, P=N.S.). Interestingly, expression of LTCC was similar in WT and KI, even after TAC OP, which is in accordance with previously published data (see discussion). In WT mice, LTCC levels were $0.21 \pm 0.06$ vs. $0.27 \pm 0.05$ (P=N.S.) for Sham vs. TAC; and in KI animals $0.27 \pm 0.04$ vs. $0.26 \pm 0.04$ (P=N.S.) for Sham vs. TAC.

A

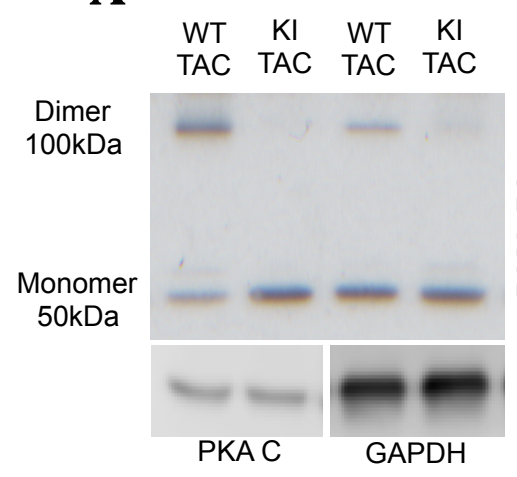

B

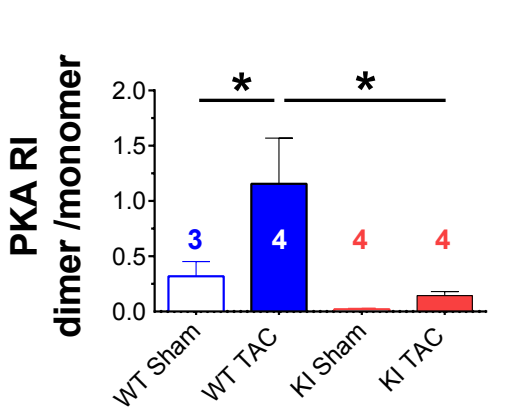

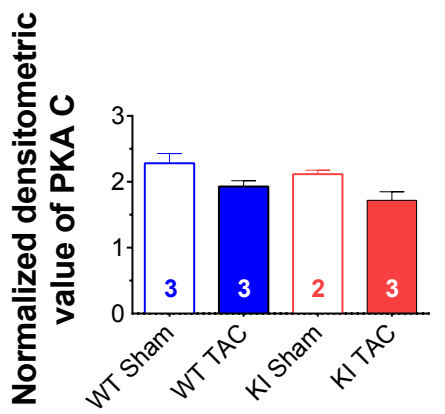

Figure 3.21. Pressure overload increases PKA RI dimer only in WT but not in KI mice. Oxidized type I PKA causes subsequent intermolecular RI dimer formation (A). Upon TAC operation, increased oxidants could modify more PKA RI subunits to form dimer compared to KI (B). However, the catalytic subunit of PKA got reduced upon TAC (C). Quantitative data represented as mean \pm standard error. At least three independent mice were used per group, ANOVA was used for statistics; * means $\mathrm{P}<0.05$.

The effects of TAC on expression and posttranslational modifications of other essential $\mathrm{Ca}^{2+}$ regulatory proteins were also investigated by western blotting (fig.3.22). Interestingly, TAC resulted in an increased PLB phosphorylation at serine 16 and reduced PLB expression in both WT and KI mice. Compared to control, TAC increased phospho-PLB/PLB from $0.66 \pm 0.13$ to $1.15 \pm 0.11(\mathrm{P}<0.05)$ in WT and from $0.38 \pm 0.08$ vs. $0.91 \pm 0.08(\mathrm{P}<0.05$, fig.3.22) in KI mice. In parallel, PLB expression was reduced from $1.86 \pm 0.17$ vs. $0.79 \pm 0.13(\mathrm{P}<0.05)$ in WT, and from $1.53 \pm 0.51$ to $1.08 \pm 0.13(\mathrm{P}=0.17$, fig.3.22) in KI. Since SERCA2a expression was unchanged, SERCA2a/PLB ratio was increased in WT (fig.3.22) suggesting that SERCA2a activity may be increased. Compared to control, SERCA2a/PLB ratio increased in WT from $0.21 \pm 0.02$ to $0.42 \pm 0.02$ ( $\mathrm{P}<0.05$, fig.3.21). In contrast, in KI mice SERCA2a/PLB levels were already increased under sham condition ( $\mathrm{P}=0.06$, fig.3.22) and did not increase further upon TAC. In KI mice, SERCA2a/PLB levels were $0.31 \pm 0.07$ vs. $0.29 \pm 0.01$ for Sham vs. TAC (P=N.S. fig.3.22). 


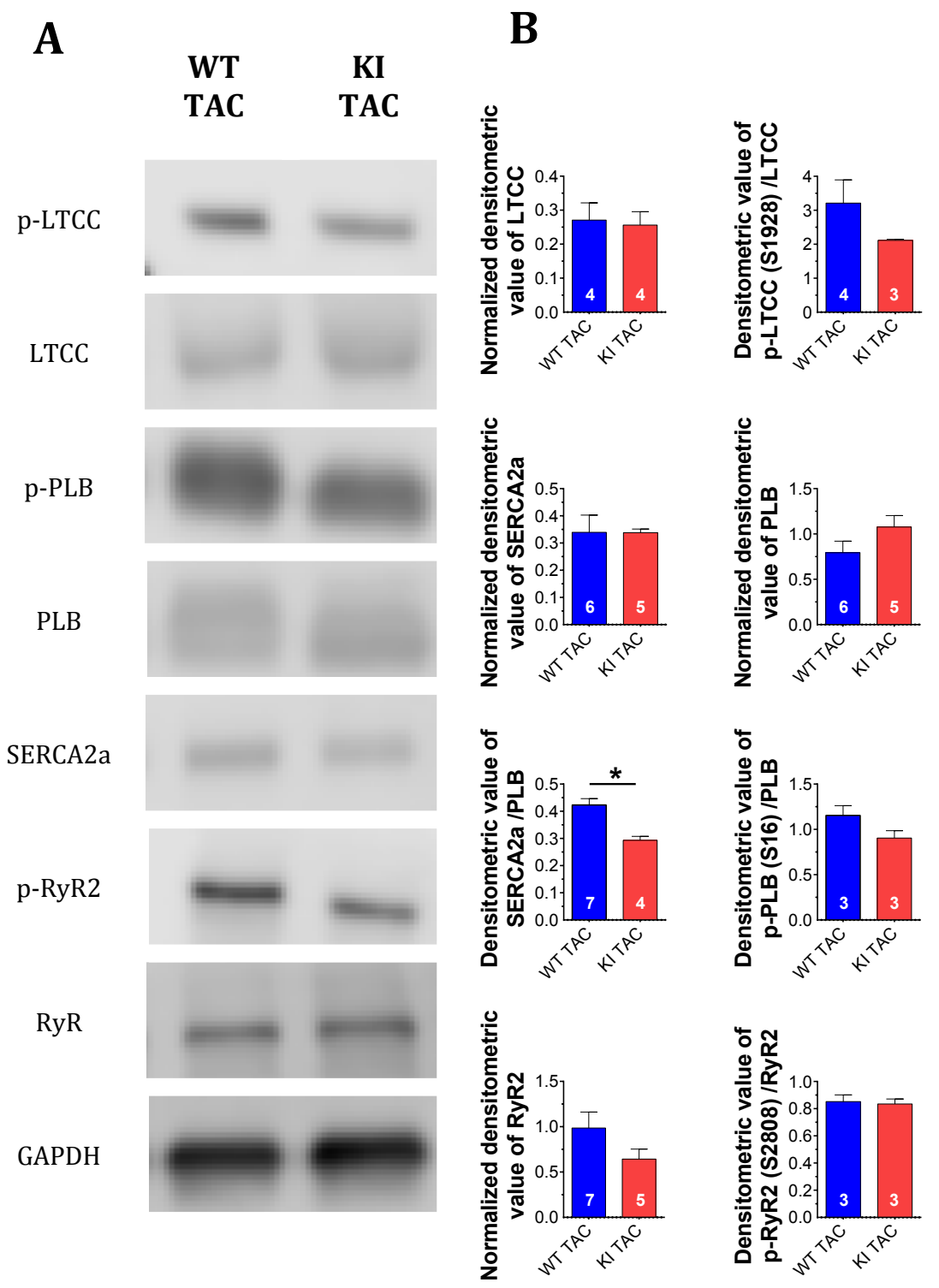

Figure 3.22. Pressure overload-mediated PKA oxidation induces phosphorylation of downstream targets. Oxidized type I PKA-mediated substrate phosphorylation after six weeks of TAC operation (A). After six weeks of TAC, expression of LTCC and SERCA2a were unchanged (B). In contrast, PLB and RyR2 were decreased. Interestingly, oxidized PKA could phosphorylate only LTCC and PLB but not RyR2. These oxidation-mediated activation of LTCC and PLB were absent in KI. Quantitative data represented as mean \pm standard error. At least three independent mice were used per group, ANOVA was used for statistics; * means $\mathrm{P}<0.05$.

Surprisingly, while RyR2 expression was unchanged after TAC in WT, it decreased significantly in KI mice (fig.3.22). RyR2 levels were $1.26 \pm 0.16$ vs. $0.99 \pm 0.18$ for Sham vs. TAC in WT (P=N.S., fig.3.22), but decreased from $1.23 \pm 0.15$ to $0.64 \pm 0.11$ in $\mathrm{KI}$ mice $(\mathrm{P}<0.05$, fig.3.22).

Interestingly, there was a significant increase in serine 2808 phosphorylation a RyR2 in WT mice ( $0.65 \pm 0.09$ vs. $0.85 \pm 0.05$, Sham vs. TAC, $P<0.05$, fig.3.22). On 
the other hand, similar to SERCA2a/PLB expression ratio, RyR2 serine 2808 phosphorylation was increased in KI already under control conditions and did not change upon TAC (fig.3.22). p-RyR2/RyR2 levels were $0.88 \pm 0.02$ vs. $0.84 \pm 0.04$ for Sham vs. TAC in KI (fig.3.22). Although there was a trend toward reduced expression of the catalytic subunit of PKA in both WT and KI mice after TAC, it did not reach statistical significance (fig.3.21). In WT, PKA C levels were $2.28 \pm 0.15$ vs. $1.93 \pm 0.09$, Sham vs. TAC, $\mathrm{P}=0.07$, and in KI mice the levels were $2.12 \pm 0.06$ vs. $1.7 \pm 0.13(\mathrm{P}=0.07$, fig.3.21).

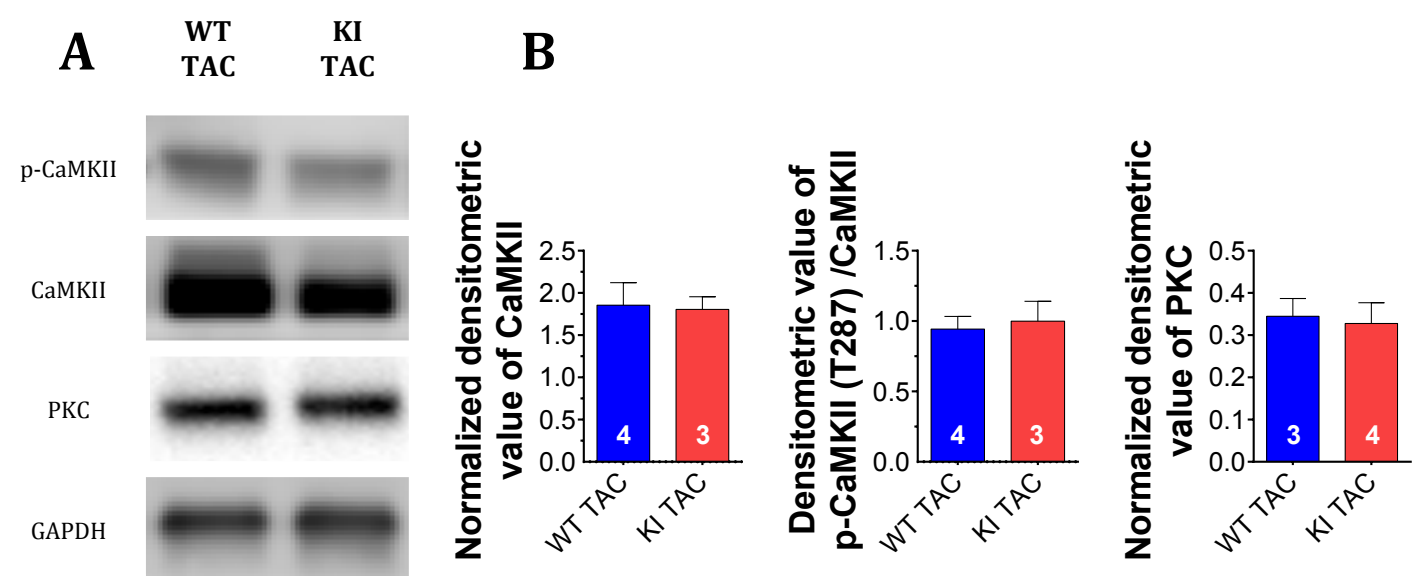

Figure 3.23. CaMKII and PKC expressions are not different in mice upon pressure overload. After six weeks of TAC, expression of CaMKII was increased; however, PKC level stayed same (A). However, autophosphorylation of CaMKII was also increased in both WT and KI TAC groups (B). Quantitative data represented as mean \pm standard error. At least three independent mice were used per group, ANOVA was used for statistics; * means $\mathrm{P}<0.05$.

In order to test if other kinases, like multifunctional CaMKII or PKC, which can also modulate EC coupling, played any role in this setting, their expression, and post-translational modifications were investigated. In accordance with previously published data, TAC increased CaMKII expression and auto-phosphorylation at threonine 287 resulting in increased CaMKII activity. In WT mice, TAC increased CaMKII levels from $1.16 \pm 0.15$ to $1.85 \pm 0.27(\mathrm{P}<0.05$, fig.3.23) and $\mathrm{p}$ CaMKII/CaMKII from $0.35 \pm 0.08$ to $0.94 \pm 0.09$, Sham vs. TAC, $\mathrm{P}<0.05$. This increase was also observed in KI mice. TAC increased CaMKII levels from $1.19 \pm 0.11$ to $1.81 \pm 0.15$ ( $\mathrm{P}=0.11$, fig.3.23) and p-CaMKII/CaMKII from $0.58 \pm 0.14$ vs. $0.99 \pm 0.14$, Sham vs. TAC $(\mathrm{P}<0.05$, fig.3.23) in $\mathrm{KI}$, although the increase in CaMKII expression did not reach statistical significance. Moreover, the expression of PKC was also not different in these mice after the TAC op (Sham vs. TAC; WT, $0.44 \pm 0.05$ vs. $0.34 \pm 0.04$; KI, $0.38 \pm 0.05$ vs. $0.33 \pm 0.05$, fig.3.23). 


\subsection{TAC surgery severely impair contractile function in mice}

\section{lacking oxidative activation of PKA}

It is apparent from chronic AngII exposure model that, lack of phosphorylation and subsequent activation of LTCC may result in aggravated contractile function upon TAC; therefore, in vivo cardiac contractility was measured using the echocardiography technique. Figure 3.24 shows original traces and quantitative mean echocardiographic data for mice that underwent TAC surgery. Doppler analysis of pressure gradients showed equal increases in afterload after TAC in both WT and KI mice (in WT, Sham vs. TAC, $3.34 \pm 1.01$ vs. $59.46 \pm 3.76 \mathrm{mmHg}, \mathrm{P}<0.05$; and KI Sham vs. TAC, $2.71 \pm 0.58$ vs. $66.13 \pm 5.48 \mathrm{mmHg}, \mathrm{P}<0.05$, fig.3.24).

A

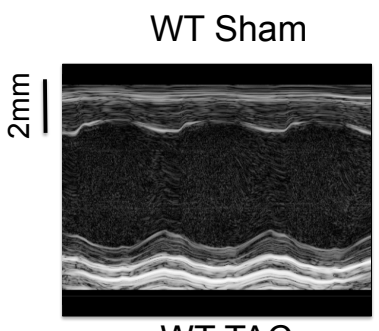

WT TAC

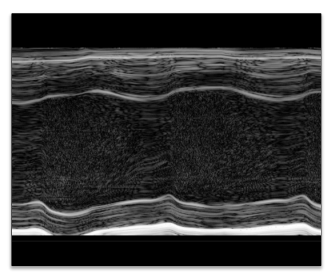

C

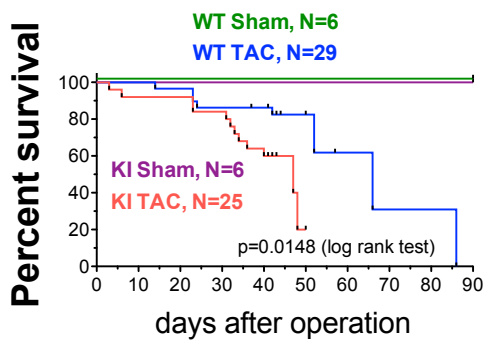

$\mathrm{KI} \mathrm{TAC}$
B
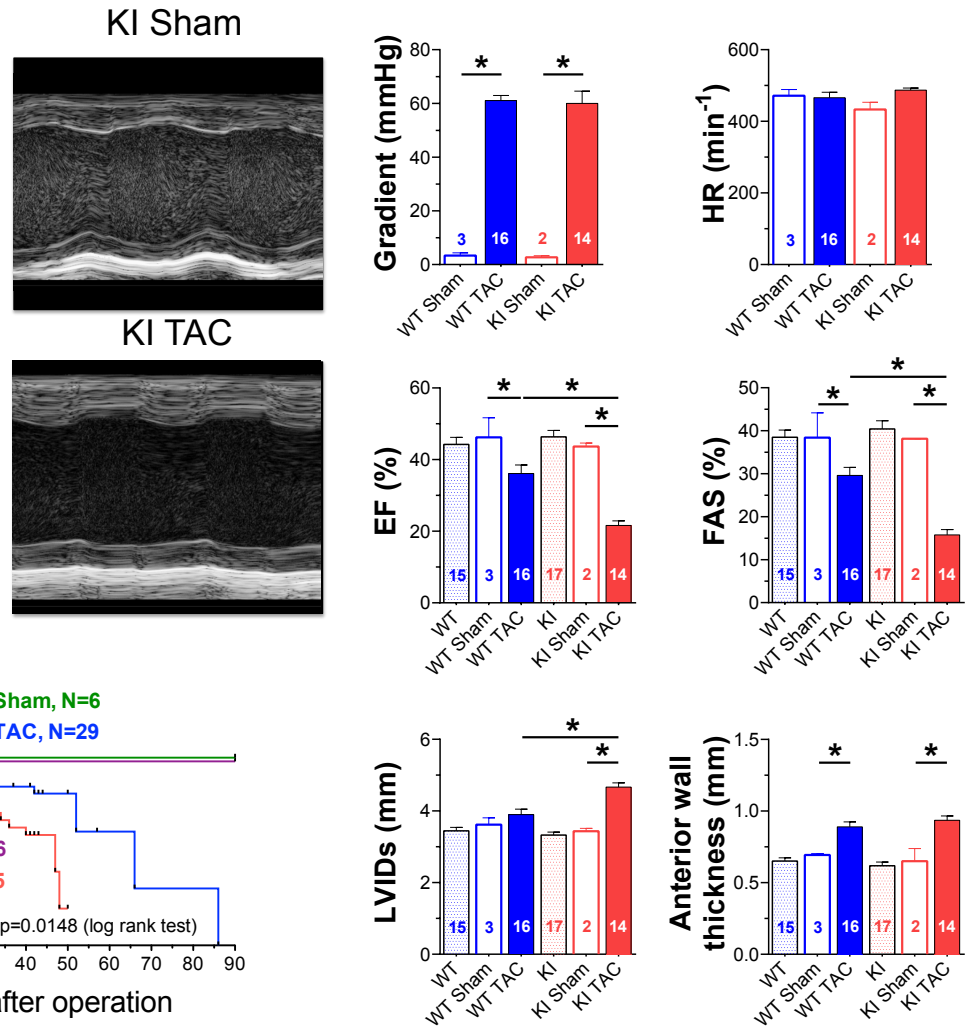

Figure 3.24. In vivo echocardiography reveal impaired cardiac contraction upon pressure overload. Representative M-mode Echocardiography revealed a similar pattern of cardiac dysfunction between WT and KI after TAC surgery, as also shown in B-mode data (A). Doppler mode echo analysis showed TAC induces sufficient pressure gradient at the site of constriction (B, top). Quantitative B-mode echo data demonstrated that both the WT and KI had reduced ejection fraction and fractional shortening upon six weeks of TAC. However, KI showed a significant reduction in cardiac function compared to control and WT mice. KI also had more severe dilatation compared to other groups as revealed by systolic left ventricular inner diameter (B, middle and lower panel). This contractile dysfunction might responsible for significantly higher mortality in KI compared to WT upon TAC (C). Quantitative data represented as mean \pm standard error. At least three independent mice were used per group, ANOVA was used for statistics; * means $\mathrm{P}<0.05$. 
In accordance with previous reports, six weeks after TAC WT mice develop an impairment of contractile function compared to sham-operated mice (fig.3.24). Both ejection fraction and fractional area shortening were significantly reduced. EF was $46.22 \pm 5.44$ vs. $36.11 \pm 2.37$, Sham vs. TAC, $\mathrm{P}<0.05$; and FAS was $38.39 \pm 5.76$ vs. $29.60 \pm 1.87$, Sham vs. TAC, $\mathrm{P}<0.05$. This TAC-dependent impairment of contractile function, however, was significantly more severe in mice lacking the oxidative activation of PKA (KI, fig.3.24). TAC decreased EF to $21.62 \pm 1.27$ vs. $43.64 \pm 0.99$ (Sham, $\mathrm{P}<0.05$ ) in $\mathrm{KI}$ mice and this reduction was significantly more pronounced compared to WT TAC $(\mathrm{P}<0.05)$. Similarly, FAS was significantly more reduced in KI upon TAC to $15.77 \pm 1.22$ ( $\mathrm{P}<0.05$ vs. WT TAC, fig.3.24). Importantly, lack of redox-dependent PKA activation increased the TAC-dependent mortality in these mice. Compared to WT mice, survival after TAC was significantly reduced in KI (fig.3.24).
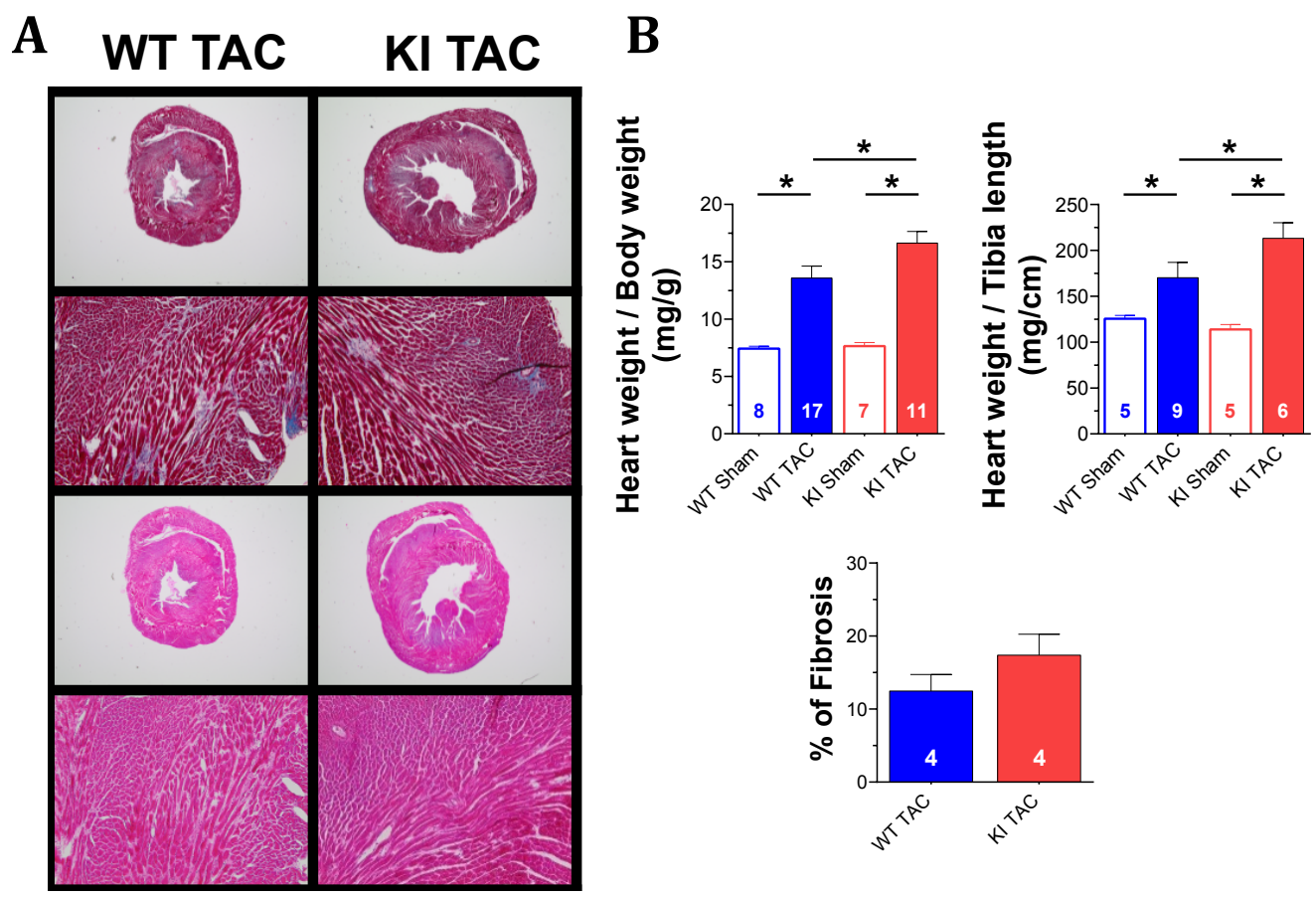

Figure 3.25. Pressure overload induces morphologic changes in the heart. Representative MTC and Hematoxylin staining of whole heart sections showed that KI had more hypertrophy than WT upon six weeks of TAC (A, left). Heart weight to body weight or heart weight to tibia length ratio also indicated a similar pattern (B). Immunohistochemical analysis showed that KI has a tendency of having more fibrosis after pressure overload compared to WT. Quantitative data represented as mean \pm standard error. At least three independent mice were used per group, ANOVA was used for statistics; * means $\mathrm{P}<0.05$.

Structural remodeling in the KI hearts were more pronounced compared to WT animals, which matches with more severe heart failure phenotype of KI mice. KI 
hearts showed significantly more dilatation of the left ventricle upon TAC. Compared to WT animals, LVIDs increased in KI upon TAC to $4.67 \pm 0.12$ vs. $3.89 \pm 0.15$ $\mathrm{mm}(\mathrm{P}<0.05)$. Also, heart weight $(\mathrm{HW})$ normalized to body weight $(\mathrm{BW})$, or tibia length (TL) was significantly more increased in KI compared to WT mice. TAC increased HW/BW to $16.63 \pm 1.01$ vs. $13.57 \pm 1.05 \mathrm{mg} / \mathrm{g}(\mathrm{KI}$ vs. WT, $\mathrm{P}<0.05)$ and HW/TL to $213.2 \pm 16.99$ vs. $170.2 \pm 16.70 \mathrm{mg} / \mathrm{cm}$ (KI vs. WT, $\mathrm{P}<0.05$, fig.3.25).

Table 3.5. Summary of echocardiographic parameters of WT and KI mice after six weeks sham- and TAC-surgery (mean \pm SEM).

\begin{tabular}{|c|c|c|c|c|}
\hline & WT Sham & WT TAC & KI Sham & KI TAC \\
\hline Number & 3 & 16 & 2 & 14 \\
\hline HR (bpm) & $471.333 \pm 17.372$ & $466.063 \pm 15.193$ & $433.000 \pm 20.000$ & $486.857 \pm 5.969$ \\
\hline BW (g) & $26.800 \pm 2.312$ & $23.825 \pm 0.833$ & $24.150 \pm 3.150$ & $22.521 \pm 0.956$ \\
\hline FS (\%) & $21.061 \pm 2.123$ & $16.055 \pm 1.425$ & $20.204 \pm 0.723$ & $6.860 \pm 0.846$ \\
\hline $\operatorname{sv}(\mu \mathrm{l})$ & $41.755 \pm 3.449$ & $37.759 \pm 2.106$ & $34.544 \pm 4.366$ & $30.553 \pm 1.675$ \\
\hline $\mathrm{CO}(\mathrm{ml} / \mathrm{min})$ & $19.799 \pm 2.321$ & $17.796 \pm 1.360$ & $14.870 \pm 1.199$ & $14.888 \pm 0.857$ \\
\hline $\mathrm{CI}(\mu \mathrm{l} / \mathrm{min} / \mathrm{g})$ & $734.812 \pm 29.460$ & $759.974 \pm 66.419$ & $619.811 \pm 31.174$ & $663.146 \pm 30.723$ \\
\hline LVIDd (mm) & $4.581 \pm 0.169$ & $4.628 \pm 0.120$ & $4.304 \pm 0.140$ & $5.007 \pm 0.102$ \\
\hline Vol s $(\mu \mathrm{l})$ & $49.731 \pm 7.733$ & $70.712 \pm 6.832$ & $44.428 \pm 3.843$ & $114.266 \pm 7.616$ \\
\hline Vol d $(\mu \mathrm{l})$ & $91.486 \pm 6.458$ & $108.470 \pm 7.125$ & $78.972 \pm 8.209$ & $144.819 \pm 8.136$ \\
\hline PWThd (mm) & $0.609 \pm 0.032$ & $0.752 \pm 0.044$ & $0.540 \pm 0.048$ & $0.722 \pm 0.031$ \\
\hline AWThs (mm) & $0.946 \pm 0.167$ & $1.161 \pm 0.048$ & $0.913 \pm 0.058$ & $1.088 \pm 0.044$ \\
\hline PWThs (mm) & $0.748 \pm 0.026$ & $0.886 \pm 0.055$ & $0.662 \pm 0.076$ & $0.802 \pm 0.030$ \\
\hline Ls (mm) & $7.140 \pm 0.187$ & $7.658 \pm 0.202$ & $7.059 \pm 0.151$ & $8.938 \pm 0.183$ \\
\hline Ld (mm) & $8.190 \pm 0.124$ & $8.461 \pm 0.153$ & $7.754 \pm 0.304$ & $9.607 \pm 0.191$ \\
\hline
\end{tabular}

In contrast, TAC increased left ventricular anterior wall thickness to similar extents in WT and KI mice suggesting that redox-dependent PKA is not involved in the regulation of left ventricular hypertrophy upon increased afterload. Compared to sham, TAC increased wall thickness to $0.89 \pm 0.03 \mathrm{~mm}$ (vs. Sham $0.69 \pm 0.01 \mathrm{~mm}, \mathrm{P}<0.05$ ) in $\mathrm{WT}$, and to $0.94 \pm 0.03 \mathrm{~mm}$ (Sham $0.65 \pm 0.09 \mathrm{~mm}$, $\mathrm{P}<0.05)$ in $\mathrm{KI}(\mathrm{P}=\mathrm{N} . \mathrm{S}$. vs. WT TAC, fig.3.24). Besides, immunohistochemical analysis showed that KI mice have a tendency to have more fibrosis compared to WT after six weeks of TAC op (WT vs. KI; $12.47 \pm 2.27$ vs. $17.38 \pm 2.86 \%$ Fibrosis, $\mathrm{P}=$ N.S., fig.25).

\subsection{Calcium channel function is severely impaired in KI mice after TAC surgery}

In order to test, if the impairment of contractile function is indeed a consequence of disturbed $\mathrm{Ca}^{2+}$ channel function, $\mathrm{I}_{\mathrm{Ca}}$ was measured in isolated ventricular cardiomyocytes isolated from mice six weeks after TAC. Analysis of the whole cell patch clamp data is shown in figure 3.26. Original traces (A) and quantitative data for current-voltage relationship revealed that TAC did not affect peak $\mathrm{I}_{\mathrm{Ca}}$ in WT cardiomyocytes. 
A
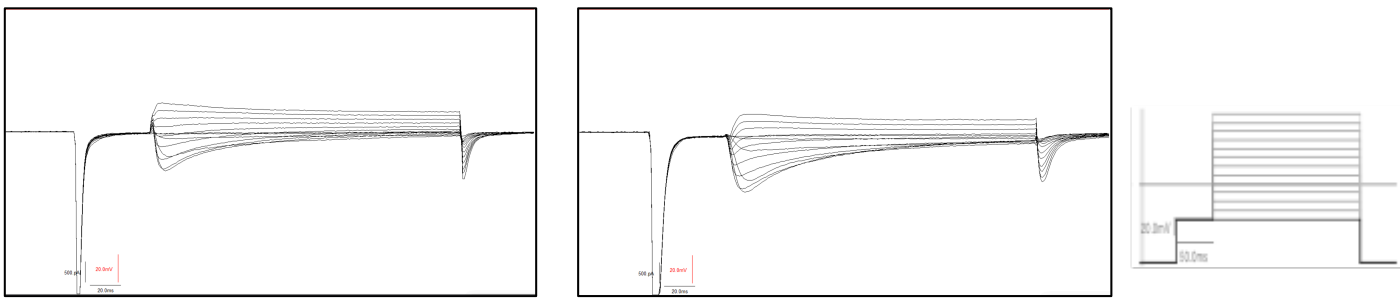

WT, membrane capacitance $160 \mathrm{pF} \mathrm{KI}$, membrane capacitance $312 \mathrm{pF}$

B

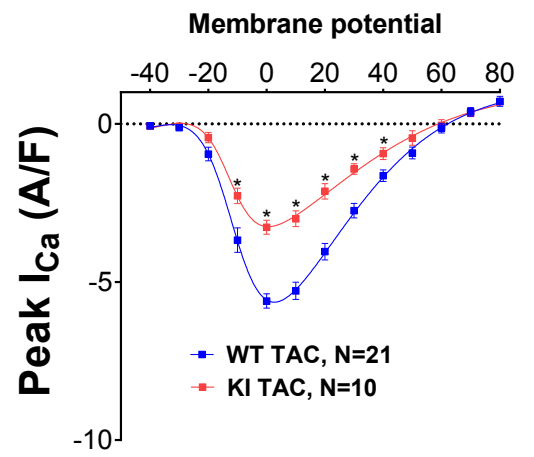

C

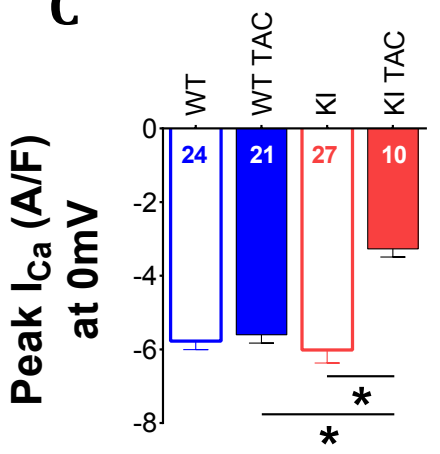

D

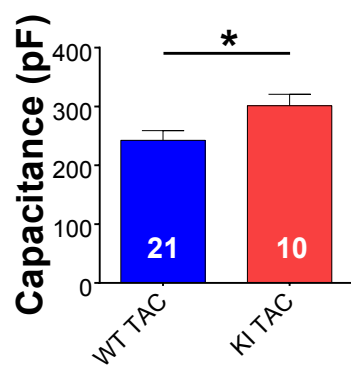

Figure 3.26. Redox-dead PKA mice have compromised calcium channel function after TAC surgery. Original whole cell patch clamp traces of WT and KI isolated ventricular cardiomyocytes upon six weeks of TAC (A). Even though KI had a large cell compared to WT, as expressed by high capacitance, the current density was lower in KI (A). Graphical representation of current-voltage protocol used in this experiment (A, right). Upon six weeks of TAC, WT maintained $\mathrm{I}_{\mathrm{Ca}}$ but KI could not (B). Compared to WT and control, KI had significantly reduced peak current at $0 \mathrm{mV}$ (C). Moreover, capacitance analysis showed that KI had high capacitance, meaning bigger cells than WT mice (D). Quantitative data represented as mean \pm standard error. At least two independent mice were used per group, ANOVA was used for statistics; * means $\mathrm{P}<0.05$.

At $0 \mathrm{mV}$, peak $\mathrm{I}_{\mathrm{Ca}}$ was $-5.77 \pm 0.24$ vs. $-5.60 \pm 0.23$ A/F (Sham vs. TAC, P=N.S., fig.3.26). In sharp contrast, TAC significantly reduced peak $\mathrm{I}_{\mathrm{Ca}}$ in cardiomyocytes lacking oxidative activation of PKA (fig.3.24). In KI mice, peak $\mathrm{I}_{\mathrm{Ca}}$ was significantly reduced from $-6.01 \pm 0.35$ to $-3.27 \pm 0.22$ A/F (Sham vs. TAC, $\mathrm{P}<0.05$, fig.3.24). This reduction, however, may be partly explained by increases in cell size. There was a significant increase in membrane capacitance in KI mice upon TAC (WT vs. KI, 201.7 \pm 8.9 vs. $295.2 \pm 14.8$ pF, $\mathrm{P}<0.05$, fig.3.26).

Surprisingly, heterozygous cys17ser PKA RI mice showed intermediate peak $\mathrm{I}_{\mathrm{Ca}}$ after six weeks of TAC compared to WT and KI TAC mice (-4.44 \pm 0.33$)$. This $\mathrm{I}_{\mathrm{Ca}}$ is significantly reduced compared to WT mice but substantially higher than KI TAC mice. Indicating that redox PKA is involved in LTCC regulation. As expected, this mice had similar baseline current $(-5.50 \pm 0.27)$, which further supports the idea 
that redox PKA is crucial under stress but not in normal conditions. Moreover, these mice also showed significantly higher survival in Kaplan-Maier analysis compared to KI TAC mice but not to WT TAC mice.

\subsection{Pressure overload reduces the force of contraction of car- diomyocytes}

In order to test, if $\mathrm{Ca}^{2+}$ channel dysregulation indeed underlies the contractile dysfunction observed in KI mice, calcium transients were measured in cardiomyocytes isolated from TAC-operated mice. There was a strong trend towards reduced $\mathrm{Ca}^{2+}$ transient amplitude in ventricular cardiomyocytes isolated from mice six weeks after TAC (fig.3.27). At $0.5 \mathrm{~Hz}$ stimulation, $\mathrm{Ca}^{2+}$ transient amplitude decreased in WT to $0.15 \pm 0.01$ (Sham $0.21 \pm 0.02$, $\mathrm{P}<0.05$, fig.3.27). Surprisingly, although there was a trend, there was no significant difference in $\mathrm{Ca}^{2+}$ transient amplitude between WT and KI cardiomyocytes after TAC. In KI cardiomyocytes, $\mathrm{Ca}^{2+}$ transient amplitude decreased to $0.12 \pm 0.03$ (Sham $0.18 \pm 0.02$, $\mathrm{P}=0.056$ vs. Sham; $\mathrm{P}=0.33$ vs. WT TAC, fig.3.27).

Interestingly, TAC slowed $\mathrm{Ca}^{2+}$ transient decay, which is a measure of SERCA2a activity. In WT cardiomyocytes, the time constant of decay, tau, increased with TAC from $0.39 \pm 0.03$ (Sham) to $0.51 \pm 0.04 \mathrm{~s}$ (P=0.24, fig.3.27). Similarly, TAC increased tau in KI from $0.45 \pm 0.05$ (Sham) to $0.75 \pm 0.13 \mathrm{~s}(\mathrm{P}<0.05$, fig.3.27). Importantly, compared to WT, the TAC-dependent increase in tau was significantly more pronounced in KI cardiomyocytes ( $\mathrm{P}<0.05$ vs. WT TAC). One reason may be KI with its significantly less favorable SERCA2a/PLB ratio, need more time to reuptake the cytosolic $\mathrm{Ca}^{2+}$ back to SR upon TAC (see above).

In accordance with reduced contractile function upon TAC, caffeine-induced $\mathrm{Ca}^{2+}$ transient amplitude (a measure of SR $\mathrm{Ca}^{2+}$ content) was significantly reduced in WT cardiomyocytes (Sham vs. TAC, $1.29 \pm 0.24$ vs. $0.79 \pm 0.11, \mathrm{P}<0.05$, fig.3.27). In accordance with baseline data (see above), caffeine-induced $\mathrm{Ca}^{2+}$ transients were reduced in KI vs. WT, and the difference was abolished after TAC surgery. Caffeine-transient amplitude in KI cardiomyocytes was $1.01 \pm 0.17$ (Sham, $\mathrm{P}=0.20$ vs. WT Sham) and was further reduced by TAC to $0.54 \pm 0.06$ (P=N.S. vs. WT TAC, 
fig.3.27). Thus, the more severely impaired contractile function of KI mice upon TAC cannot be explained by changes in SR $\mathrm{Ca}^{2+}$ content but may be due to impaired LTCC function.

\section{A}
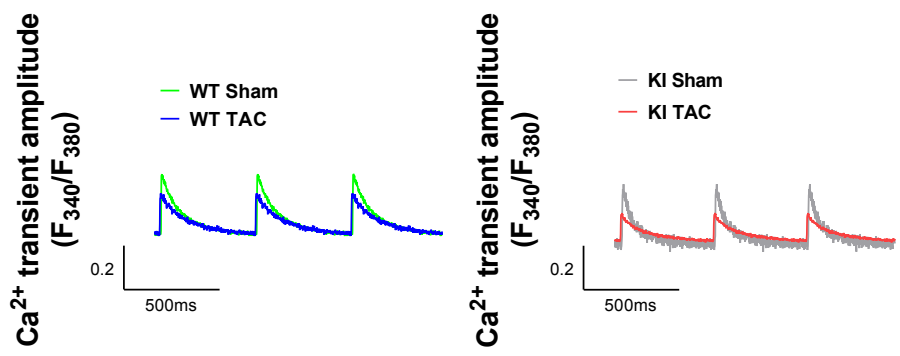

B
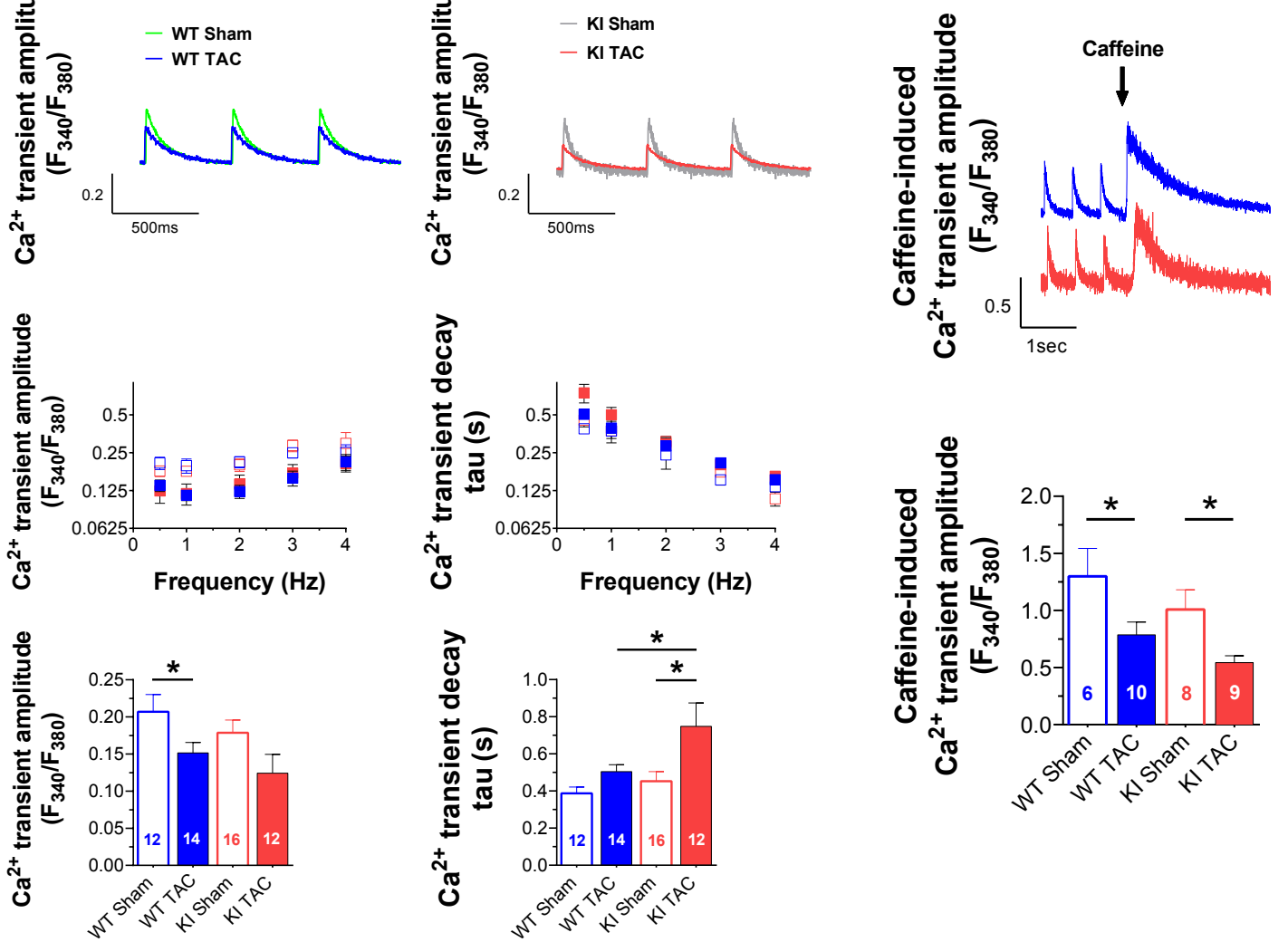

Figure 3.27. Pressure overload impairs calcium handling of isolated cardiomyocytes. Original traces and quantitative analysis of $\mathrm{Ca}^{2+}$ transients (A, top). Representative transients at $0.5 \mathrm{~Hz}$ showed that both WT and KI had reduced $\mathrm{Ca}^{2+}$ transient amplitude and delayed reuptake of $\mathrm{Ca}^{2+}$ to the SR upon six weeks of TAC (A, bottom). Quantitative mean data showed that the reduction in $\mathrm{Ca}^{2+}$ transient amplitude was more pronounced in $\mathrm{KI}$ compared to WT and control groups. In addition, reuptake of $\mathrm{Ca}^{2+}$ back to SR was much slower in KI compared to WT (A, below). This was further reflected from the caffeine-induced $\mathrm{Ca}^{2+}$ transient data, where $\mathrm{KI}$ showed less SR Ca ${ }^{2+}$ content compared to controls and also to WT TAC mice (B). Quantitative data represented as mean \pm standard error. At least three independent mice were used per group, ANOVA was used for statistics; * means $\mathrm{P}<0.05$.

\subsection{KI mice have more arrhythmic propensity upon TAC sur- gery compared to WT}

In order to test, if the more severely impaired left ventricular remodeling of KI mice after TAC also translates into increased propensity for ventricular arrhythmias, programmed electrical stimulation was used. Baseline ECG parameters are shown in Figure 3.28 and Table 3.6. 
A

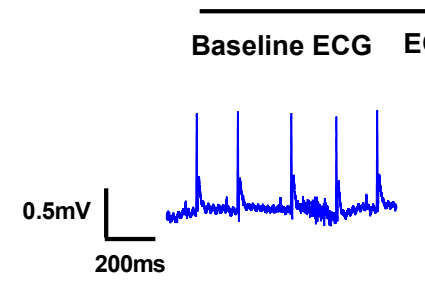

B

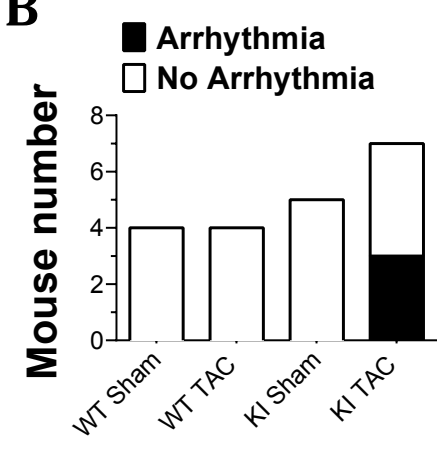

WT TAC

ECG upon burst stimulation

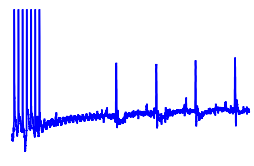

KI TAC

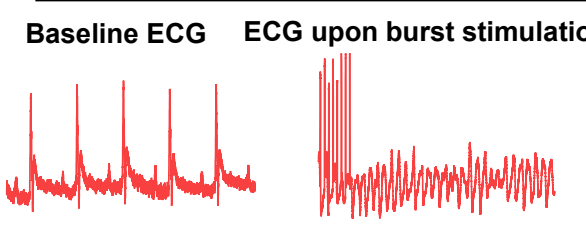

C

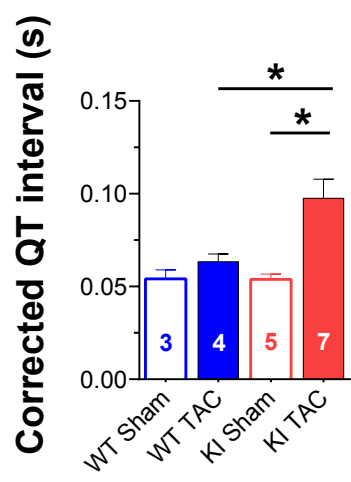

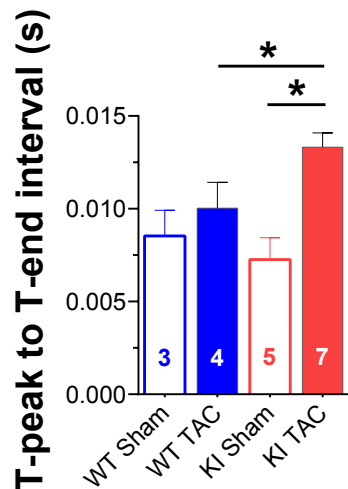

Figure 3.28. Pressure overload increases the arrhythmogenic vulnerability in KI mice. Original ECG traces during programmed electrical stimulation in vivo showed altered ECG parameters in KI after six weeks of TAC compared to WT (A, left). Interestingly, in TAC-operated WT mice, burst stimulation did not induce arrhythmias (A, right). In contrast, in TAC-operated KI mice, the same protocol induced polymorphic VT. Colum graph showed numbers of mice showing arrhythmia upon programmed electrical stimulation (B). Resting ECG parameter (corrected QT [QTc] interval, T peak T end [TT]) showed KI had significantly higher QTc and TT interval compared to WT TAC and control groups (C). At least three independent mice were used per group, ANOVA was used for statistics; * means $\mathrm{P}<0.05$.

Mean data showed that TAC increased QTc in both WT (Sham vs. TAC, $54.10 \pm 4.84$ vs. $63.33 \pm 4.18 \mathrm{~ms}, \mathrm{P}=0.52$ ) and KI mice (Sham vs. TAC, $53.83 \pm 2.92$ vs. 97.57 $\pm 10.29 \mathrm{~ms}, \mathrm{P}<0.05$, fig.3.28). However, the increase in QTc was significantly more pronounced in KI mice ( $\mathrm{P}<0.05$ vs. WT, fig.3.28). A similar tendency was observed for the T peak-Tend interval, which was increased after TAC in both WT (Sham vs. TAC, $8.54 \pm 1.39$ vs. $10.03 \pm 1.40 \mathrm{~ms}, \mathrm{P}=0.43$ ) and KI mice (Sham vs. TAC, $7.26 \pm 1.18$ vs. $13.32 \pm 0.76 \mathrm{~ms}, \mathrm{P}<0.05$ vs. Sham and $\mathrm{P}<0.05$ vs. WT TAC, fig.3.28).

Besides, programmed electrical RV apex stimulation using a burst protocol could only induce ventricular tachyarrhythmia in KI but not in WT mice upon TAC. Interestingly, none of the sham-operated mice showed any arrhythmia after programmed electrical stimulation. 
Table 3.6. Summary of electrocardiographic parameters of WT and KI mice after six weeks of sham- and TAC-surgery (mean \pm SEM).

\begin{tabular}{|l|c|c|c|c|}
\hline & WT Sham & WT TAC & KI Sham & KI TAC \\
\cline { 2 - 5 } Number & 3 & 4 & 5 & 7 \\
RR Interval (s) & $0.116 \pm 0.0049$ & $0.137 \pm 0.0107$ & $0.127 \pm 0.0054$ & $0.122 \pm 0.0033$ \\
Heart Rate (BPM) & $521.167 \pm 21.6738$ & $447.525 \pm 33.3085$ & $476.800 \pm 20.4684$ & $495.814 \pm 13.4287$ \\
PR Interval (s) & $0.037 \pm 0.0015$ & $0.041 \pm 0.0015$ & $0.037 \pm 0.0024$ & $0.035 \pm 0.0014$ \\
P Duration (s) & $0.008 \pm 0.0011$ & $0.008 \pm 0.0011$ & $0.008 \pm 0.0007$ & $0.008 \pm 0.0006$ \\
QRS Interval (s) & $0.007 \pm 0.0005$ & $0.010 \pm 0.0003$ & $0.009 \pm 0.0008$ & $0.012 \pm 0.0019$ \\
QT Interval (s) & $0.018 \pm 0.0013$ & $0.023 \pm 0.0017$ & $0.019 \pm 0.0008$ & $0.034 \pm 0.0033$ \\
JT Interval (s) & $0.011 \pm 0.0017$ & $0.014 \pm 0.0019$ & $0.010 \pm 0.0011$ & $0.022 \pm 0.0018$ \\
P Amplitude (mV) & $0.140 \pm 0.0272$ & $0.138 \pm 0.0049$ & $0.103 \pm 0.0169$ & $0.137 \pm 0.0161$ \\
Q Amplitude (mV) & $-0.026 \pm 0.0115$ & $-0.035 \pm 0.0085$ & $-0.014 \pm 0.0097$ & $-0.011 \pm 0.0058$ \\
R Amplitude (mV) & $0.850 \pm 0.1297$ & $0.889 \pm 0.0364$ & $0.889 \pm 0.115$ & $1.324 \pm 0.3049$ \\
S Amplitude (mV) & $-0.258 \pm 0.0968$ & $-0.105 \pm 0.0743$ & $-0.335 \pm 0.0594$ & $-0.167 \pm 0.0668$ \\
T Amplitude (mV) & $0.201 \pm 0.0456$ & $0.237 \pm 0.0322$ & $0.127 \pm 0.0380$ & $0.006 \pm 0.1271$ \\
\hline
\end{tabular}

\subsection{Human diseased patients show oxidative activation of PKA}

In order to test, if oxidative activation of PKA is also observed in diseased human hearts, PKA RI dimer formation was measured in right atrial appendage biopsies from patients who underwent coronary artery bypass grafting.

A

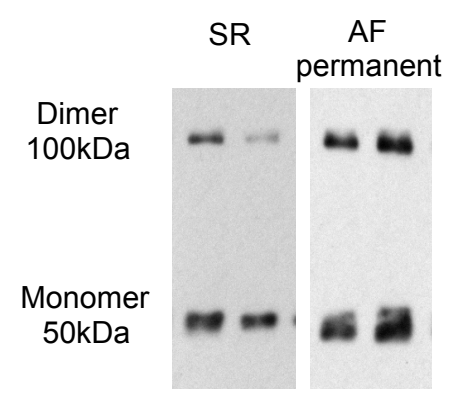

B

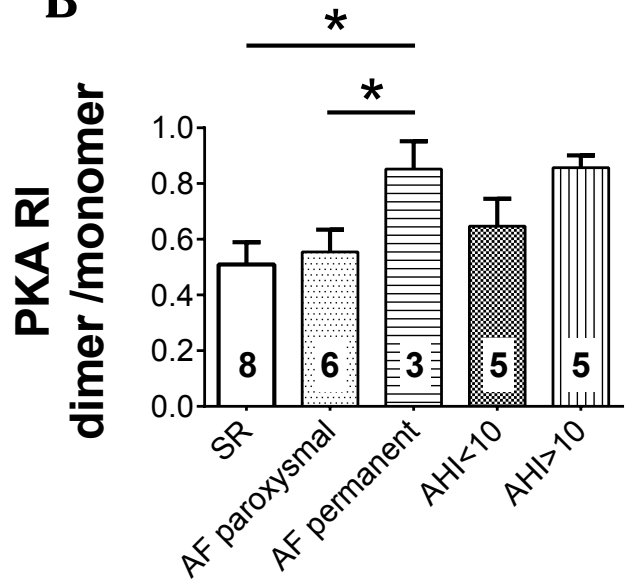

Figure 3.29. Oxidation of PKA regulatory subunit at different disease stages. Original western blot showed PKA RI dimer formation in sinus rhythms (SR) and permanent atrial fibrilation (AF) patient samles (A). In sinus rhythms and mild disease conditions like paroxysmal AF or mild sleep apnea patient body maintain a certain level of oxidized PKA. Permanent AF or severe sleep apnea patient produced significantly higher oxidized PKA compared to sinus rhythm (B). Quantitative data represented as mean \pm standard error. At least three independent patient samples were used per group; ANOVA was used for statistics; * means $\mathrm{P}<0.05$.

Figure 3.29 shows that RI dimer/monomer ratios were significantly increased in patients with permanent atrial fibrillation or sleep-disordered breathing, indicated by increased apnea-hypopnea index (AHI greater than 10), compared to patients with sinus rhythm and no history of sleep-disordered breathing, respectively. Surprisingly, despite previous data showing that patients with paroxysmal atrial fibrillation have increased ROS levels in the atrium, no increases RI dimer formation was observed in this cohort of patients (fig.3.29). 


\section{Chapter4 Discussion}


In this study, the physiologic and pathophysiologic role of redox-activated PKA for cardiac excitation-contraction coupling has been evaluated. I show here that

1) AngII- and TAC-mediated ROS can cause type I PKA dimer formation and subsequent PKA activation,

2) Oxidized PKA can specifically phosphorylate LTCC and modulate its function,

3) Mice lacking this pathway display normal basal phenotype, and

4) Inability to maintain this particular pathway leads to severe contractile dysfunction and arrhythmia under chronic AngII infusion and upon TAC.

These results suggest that redox-activated PKA may be an important novel drug target for the treatment of patients with arrhythmias and heart failure.

\subsection{Redox-dead PKA mice have no distinct phenotype at base- line}

Brennan and colleagues showed that type I PKA can be oxidatively activated without the help of its classical activator cAMP. This activation involves intermolecular dimer formation of PKA regulatory subunits, which subsequently release catalytic subunits to mediate substrate phosphorylation for ultimate physiologic effects. However, the relevance of this pathway for the cardiac function was completely unknown. Thus, a novel mouse line has been used in this study, which has only a single amino-acid substitution compared to its WT littermates. This point mutation changes cysteine at position 17 to serine. This alteration has no effect on protein structure, conformation or conventional agonist (cAMP) binding for downstream signaling (Brennan et al., 2006; Burgoyne et al., 2015). However, these mice are only unable to activate type I PKA upon oxidation via intermolecular dimer formation. Our group recently showed that these mice are incapable of maintaining angiogenesis upon hind limb ischemia, although exhibiting normal phenotype at baseline condition (Burgoyne et al., 2015). Since PKA is an important regulator of cardiac function under physiologic conditions and during heart failure development (Bers, 2001), this novel mouse model could reveal functional significance of redox-activated PKA in the heart. 
Similar to Burgoyne's report, in this project isolated ventricular cardiomyocytes from KI mice show comparable cardiac contractile parameters, such as $\mathrm{Ca}^{2+}$ transient, spontaneous SR $\mathrm{Ca}^{2+}$ release or $\mathrm{Ca}^{2+}$ transient decay, compared to cardiomyocyte expressing WT PKA at baseline condition. However, at baseline, KI cardiomyocytes have lower SR calcium content and higher peak $\mathrm{I}_{\mathrm{Ca}}$ compared to WT cardiomyocytes. These results are in agreement with the previous report, where WT had similar $\mathrm{I}_{\mathrm{Ca}}$, around $-6 \mathrm{~A} / \mathrm{F}$ at baseline (Leroy et al., 2011). Thus, KI mice with increased $\mathrm{I}_{\mathrm{Ca}}$ partially compensate $\mathrm{Ca}^{2+}$ transients. Interestingly, results from this study show that at baseline KI cardiomyocytes maintained a higher level of intracellular cAMP compared to WT cardiomyocytes, implicating the role of oxidized PKA in baseline $\mathrm{Ca}^{2+}$ handling. Bryant et al. reported the role of PKA also in maintaining $\mathrm{I}_{\mathrm{Ca}}$ (Bryant et al., 2014). However, higher cAMP concentrations in KI PKA correlates with baseline LTCC phosphorylation, where KI mice showed more phosphorylation compared to WT animals. So, KI animals maintain a higher level of cAMP, which may compensate its inability to activate PKA via oxidation. All these data strengthen the idea that at physiologic condition both cAMP and oxidized PKA is required to maintain $\mathrm{I}_{\mathrm{Ca}}$ and $\mathrm{Ca}^{2+}$ transients. This is further confirmed by the findings of Andersson et al., who showed that antioxidant $\mathrm{N}$-acetyl cysteine, NAC, can decrease the $\mathrm{I}_{\mathrm{Ca}}$ and $\mathrm{Ca}^{2+}$ transients compared to their baseline level (Andersson et al., 2011). However, as a result of this compensated cAMP response, no apparent baseline contractile abnormalities are detected in WT and KI mice with echocardiography.

\subsection{Stress induces similar oxidant production in both WT and KI mice}

Stress conditions induce oxidant production in the cardiomyocytes. However, under chronic stress situation, an excess oxidant may cause structural and electrical remodeling of the heart, contributing to cardiomyopathy, fibrillation, and arrhythmia (Youn et al., 2013). It has been reported that level of AngII is getting higher under stress conditions (Bers, 2001). Thus, AngII has been used in this study to mimic stressed conditions to the cell and also to the whole heart. Upon acute exposure, AngII induces a significantly higher amount of oxidant (ROS) under in vitro conditions. Recent reports confirmed this finding, where AngII can 
increase oxidant production in the vascular smooth muscle cell and also in cardiomyocytes (Seshiah et al., 2002; Yasunari et al., 2002). AngII-induces significantly higher mitochondrial oxidant production compared to control cardiomyocytes in acute settings, as revealed by MitoSOX, a superoxide detector. This result shows that AngII-mediated oxidant production is dependent on mitochondria. Maack et al. proposed two possible mechanisms for this oxidant production: (1) increased superoxide generation at the mitochondrial electron transport chain, ETC; and (2) decreased the elimination of oxidants in the mitochondrial matrix (Maack and Bohm, 2011). Interestingly, this mitochondrial oxidant production is also dependent on the membrane-bound NOX2 enzyme, as shown by gp91phox KO mice. These mice could not produce mitochondrial oxidant upon AngII exposure. Thus, it may implicate that cytosolic ROS is responsible for mitochondrial ROS production and vice versa. Brandes et al. and Zorov et al. termed this type of phenomenon as ROS-induced ROS release (RIRR) event (Brandes, 2005; Zorov et al., 2000). They found that upon stimulation, mitochondrial ROS could induce cytosolic ROS production and vice versa. Wen et al. summarized three possible mechanisms for RIRR: (1) ROS-mediated mitochondrial depolarization via activation of the mitochondrial permeability transition pore (MPTP) generates a burst of ROS from ETC; (2) increased ROS triggers opening of the mitochondrial membrane anion channels, which causes a brief increase in mitochondrial ETCderived ROS; and (3) cytosolic ROS stimulates the opening of mitochondrial ATPsensitive potassium (mitoKATP) channels and induces ROS production (Brady et al., 2006; Daiber, 2010; Wen et al., 2012; Zorov et al., 2006). It has been reported that AngII can trigger RIRS via all these three mechanisms (Zhang et al., 2007).

A cytosolic oxidant detector, CellROX is used in this study to correlate cytosolic ROS production to this increased mitochondrial oxidants production. Experiments with CellROX show similar results, where AngII induced significantly higher oxidant production upon acute exposure. In a similar study, Wagner et al. presented AngII-mediated cytosolic ROS production using an adenoviral construct (Wagner et al., 2014). Many reports indicated NOX, with its different isoforms, as a primary source of this cytosolic oxidant production (Brandes and Kreuzer, 2005). Among the NOX isoforms, NOX2 and 4 are predominant in the 
cardiomyocytes. Brandes et al. showed, either NOX2 or 4 alone or in combination are sufficient to produce enough cytosolic oxidants. Hence, it may well be that both NOX2 and 4 along with the mitochondrial electron transport chain are involved in AngII-mediated oxidant production (Brandes and Kreuzer, 2005; Rajagopalan et al., 1996; Xiao et al., 2002).

In vivo implantation of osmotic minipumps containing AngII show comparable results as found in ex vivo condition. Infusions of AngII (1mg/kg/day) for two weeks increase blood pressure significantly in both WT and KI mice. Results show that upon in vivo AngII infusion both WT and KI animals produced significantly higher oxidant compared to baseline. Similar to these findings, it has been reported that AngII-mediated increase in systolic blood pressure and upregulation of vascular ROS production, can be blocked by losartan, an AngII receptor inhibitor (Rajagopalan et al., 1996). Besides, Virdis et al. showed AngIIdependent effects in small resistant arteries could be prevented by the NADPH oxidase inhibitor apocynin (Virdis et al., 2004). Laursen et al. showed in rat that this AngII-induced hypertension and increased in blood pressure are due to degradation of endothelium-derived NO, possibly by increased superoxide levels. However, treatment with liposome-encapsulated SOD abolished this increase in blood pressure, indicating the involvement of AngII-induced ROS (Laursen et al., 1997).

Moreover, transverse aortic constriction (TAC) can also increase cytosolic oxidant production as visualized by CellROX. After six weeks of TAC, cardiomyocytes from both WT and KI animals produced a high amount of oxidant compared to baseline and sham-operated mice, which correlate well with previous reports (Schwarzer et al., 2014). Thus, although the role of PKA in controlling cellular ROS has been reported previously, the results listed above indicate that redox-active PKA is not involved in AngII- and TAC-induced ROS production (Chaves et al., 2009; Kim et al., 2007). 


\subsection{Oxidant-induced PKA activation is absent in KI mice}

Oxidative activation of PKA is relative to intermolecular dimer formation of PKA type I regulatory subunits. Isolated cardiomyocytes expose to $\mathrm{H}_{2} \mathrm{O}_{2}$, show increased number of RI dimers compared to control, which demonstrates oxidation of type I PKA. A similar pattern of dimer formation is also observed upon perfusion of AngII to whole mice hearts. AngII induces significantly higher dimer formation in WT mice compared to control. As expected, KI mice cannot produce this dimer because of its absence of $\mathrm{C} 17$ residue. These results indicate that $\mathrm{KI}$ mice are the appropriate models to identify functional significance of redoxactive PKA in heart. To test this hypothesis WT and KI mice are used for in vivo AngII infusion and TAC surgery to evaluate oxidation-induced dimer formation. AngII- and pressure overload-induce similar amount of oxidant in both the mice, which causes, however, significantly higher dimer formation in WT but not in KI compared to their control littermates. Burgoyne et al. reported similar results, where VEGF-induced oxidant can mediate dimer formation only in WT but not in KI (Burgoyne et al., 2015). Moreover, they have reported that VEGF-mediated oxidative activation of PKA can phosphorylate and activate ERK signaling in aortic endothelial cells. Interestingly, results from this study indicate that AngII- or TAC-induced oxidation can increase PKA activation and downstream LTCC phosphorylation in cardiomyocytes. These implicated the involvement of different signaling pathways downstream to oxidative PKA activation; for example, in vascular endothelial cells, oxidative PKA mediates its role via ERK, whereas, in cardiomyocytes, via LTCC modulation.

\subsection{Oxidant-induced modulation of cytoplasmic $\mathrm{Ca}^{2+}$ transients}

In this study, it is found that both chronic AngII infusion and pressure overload induces sufficient oxidant production in WT and KI mice, which ultimately impairs cardiac contraction. As reveal by $\mathrm{Ca}^{2+}$ transient analysis, both acute exposure and chronic infusion of AngII can increase $\mathrm{Ca}^{2+}$ transient amplitude in WT cardiomyocytes but lowers $\mathrm{Ca}^{2+}$ transient amplitude in KI mice compare to control. Whole cell patch clamp technique reveals that KI mice have lower peak $\mathrm{I}_{\mathrm{Ca}}$ compared to WT upon AngII, which may explain the redundancy in the $\mathrm{Ca}^{2+}$ transients. Moreover, stress-induced (via AngII) I $\mathrm{Ca}_{\mathrm{Ca}}$ change in WT and KI cardiomyo- 
cytes are gone with H89, showing PKA is involved here. Interestingly, baseline increase in the cAMP level of KI is diminished upon AngII, supporting the inability of KI to maintain even the baseline $\mathrm{I}_{\mathrm{Ca}}$ level. However, both the WT and KI cardiomyocytes have reduced SR $\mathrm{Ca}^{2+}$ content upon acute and chronic AngII exposure, with almost unaltered $\mathrm{Ca}^{2+}$ transient decay. Thus, WT cardiomyocytes with its ability to increase $\mathrm{I}_{\mathrm{Ca}}$ upon AngII-mediated oxidative stress can maintain the $\mathrm{Ca}^{2+}$ transients whereas $\mathrm{KI}$ is unable to do so, maybe because of their inability to oxidatively active PKA. Spark analysis reveals that both WT and KI cardiomyocytes have similar spark frequency upon AngII, indicating that RyR2 does not play a prominent role in these settings. This result is in agreement with our previous report showing the involvement of CaMKII in spark regulation (Wagner et al., 2014). Western blot analyses also showed that WT mice could increase stress-induced LTCC phosphorylation but KI animals could not. Involvement of PKA type I in LTCC regulation was documented long ago by Burton et al., who showed that type I could attach and modulate LTCC even in the absence of type II PKA (Burton et al., 1997). Although reports have been shown that LTCC can be phosphorylated by both PKA and PKC at S1928 position (Aiello and Cingolani, 2001), Ichiyanagi et al. showed a PKC-independent increase in I $I_{C a}$ upon AngII via AT1 receptor (Ichiyanagi et al., 2002). Our data indicated that this PKCindependent increase might be mediated by oxidized PKA. Bers and his colleagues mentioned that upon phosphorylation the open probability of pore forming $\alpha_{1 \mathrm{c}}$ subunit gets higher as well as the activation potential shifted towards more negative membrane potentials (Bers, 2001). Thus, at a certain time, more calcium channels can be activated for a longer period of time. In accordance, acute AngII exposures to WT cardiomyocytes show a negative shift of the activation curve but not in KI. Therefore, this increase in LTCC phosphorylation by PKA may help WT mice to maintain enough $\mathrm{I}_{\mathrm{Ca}}$ and $\mathrm{Ca}^{2+}$ transient for sufficient force generation.

Moreover, WT animals have significantly higher phosphorylation of PLB at S16 compared to control and KI mice upon AngII treatment, meaning that the $\mathrm{Ca}^{2+}$ transient decay is faster in WT compared to KI mice. However, this is not the case as revealed by the tau analysis. Higher $\mathrm{Ca}^{2+}$ transients and $\mathrm{I}_{\mathrm{Ca}}$ in WT mice 
can explain this discrepancy, indicating that a higher amount of $\mathrm{Ca}^{2+}$ in the cytoplasm requires more time for its reuptake by the SR. This is further supported by the fact that both WT and KI mice had similar SERCA2a to PLB ratio, indicating both have equally efficient systems for $\mathrm{Ca}^{2+}$ reuptake.

However, upon pressure overload-mediated stress both WT and KI mice show reduced $\mathrm{Ca}^{2+}$ transient amplitude, less SR content but an increase in $\mathrm{Ca}^{2+}$ decay time. Interestingly, upon TAC surgery, $\mathrm{Ca}^{2+}$ transients, and SR $\mathrm{Ca}^{2+}$ contents were even further reduced in KI animals compared to WT. These results correlate with $\mathrm{I}_{\mathrm{Ca}}$ data, which show that KI has significantly reduced $\mathrm{I}_{\mathrm{Ca}}$ compared to control and WT mice. Besides, KI animals have a more compromised $\mathrm{Ca}^{2+}$ reuptake capacity, tau, compared to control. All these $\mathrm{Ca}^{2+}$ handling inabilities are correlated with western blot analysis, which shows WT mice can increase LTCC phosphorylation to maintain the transients. The inability of KI animals to phosphorylate LTCC along with reduced RyR2 may be responsible for reduced $\mathrm{Ca}^{2+}$ transients in $\mathrm{KI}$ mice upon TAC. Furthermore, WT expressing more SERCA2a, along with an increase in PKA-mediated PLB phosphorylation, can reuptake the cytoplasmic $\mathrm{Ca}^{2+}$ more quickly after TAC surgery. In contrast to chronic AngII model, KI mice who have less SERCA2a but moderate PLB phosphorylation, showing severe $\mathrm{Ca}^{2+}$ handling impairment compared to WT mice after TAC, which already indicates a heart failure phenotype in KI animals (Beuckelmann et al., 1992; He et al., 2001).

\subsection{KI mice show decreased cardiac contractility upon stress}

All these in vitro $\mathrm{Ca}^{2+}$ handling impairments suggest that $\mathrm{KI}$ mice could have compromised cardiac function upon stress compared to WT mice. Data from other groups showed that AngII can mediate several effects via directly modifying cardiomyocytes or, indirectly, increasing blood pressure (Mazzolai et al., 2000). AngII significantly increases blood pressure in both WT and KI mice and induces similar morphological changes as visualized by MTC and H/E staining. Echocardiography shows KI animals have much compromised cardiac function compared to WT upon AngII exposure. While WT mice could maintain similar EF, FAS, CO and CI compared to control, the heart function of KI animals declined significantly compared to control. Dai et al. found that upon four weeks AngII in- 
fusion WT mice had only a mild increase in FS compared to control, which is comparable to our study (Dai et al., 2011b). However, EF and FAS are more reduced in KI animals compared to WT mice, although, not significant. A similar trend is also observed in cardiac output and cardiac index, which are much lower in KI mice compared to WT animals. However, both WT and KI animals have similar dilatation upon AngII; whereas WT have more hypertrophy than KI mice. The ratio of heart weight and body weight also show that WT mice had more hypertrophy than KI animals upon chronic AngII infusion. This AngII-induced hypertrophy is correlated well with previous data showing that AngII could increase the cell size, gene transcription and translation of cardiomyocytes under cell culture conditions (Miyata and Haneda, 1994; Sadoshima and Izumo, 1993a, b). Moreover, it has been reported that mice overexpressing catalase targeted to mitochondria were resistant to cardiac hypertrophy, fibrosis and mitochondrial damage induced by AngII (Dai et al., 2011a; Dai et al., 2011b). This evidence revealed that AngII-mediated ROS is involved in hypertension-induced cardiac hypertrophy. KI mice, which cannot oxidize PKA upon these increased oxidants, have less hypertrophy to compensate for the AngII-mediated stress, meaning that ROS may induce hypertrophy via oxidative PKA signaling pathway.

In contrast, pressure overload-induce more hypertrophy in KI compared to WT animals as shown in heart weight to body weight ratio and heart weight to tibia length ratio. Reports have been shown that several components of the reninangiotensin system, including angiotensinogen, angiotensin-converting enzyme (ACE), and AngII, were increased in the ventricle in response to pressure overload (Dostal et al., 1992; Schunkert et al., 1990). Moreover, Baker et al. showed that pressure overload-induced left ventricular hypertrophy could be ameliorated by feeding the animal with ACE inhibitor Enalapril maleate using rat in vivo model (Baker et al., 1990). Other reports also showed that not only the reninangiotensin system but also multiple pathways, for example, CaMKII, MAPK, HDAC are additionally involved in the pressure overload-induced hypertrophic process. This perception is in agreement with the reports, which have shown that abdominal aortic constriction in $\mathrm{AT}_{1} \mathrm{~A}$ receptor knockout mice produced cardiac hypertrophy independent of the $\mathrm{AT}_{1}$ receptor (Hamawaki et al., 1998; 
Harada et al., 1998). Thus, the additional pathways involved in pressure overload may help to explain the differences in the hypertrophic response between WT and KI mice in these two models.

The enhanced hypertrophy in KI mice upon TAC matches well with $\mathrm{Ca}^{2+}$ transient and $\mathrm{I}_{\mathrm{Ca}}$ data, which showed that KI mice have impairment in $\mathrm{Ca}^{2+}$ handling. Goonasekara et al. also demonstrated that reduced LTCC activity is associated with cardiac hypertrophy, which is mediated by neurohormonal stress and NFAT-signaling (Goonasekera et al., 2012). Moreover, echocardiography revealed that KI mice have severely reduced contractile function as shown by ejection fraction and fractional area shortening. Thus, under stress conditions, KI animals with their less $\mathrm{Ca}^{2+}$ handling capability, initiate severe hypertrophic signaling to maintain sufficient cardiac contractions, but ultimately fail. These data provide further proof that redox-activated PKA is required to maintain proper cardiac contraction upon stress.

\subsection{Activation of CaMKII is similar in both WT and KI mice upon oxidative stress}

CaMKII is a well-reported enzyme for cardiac pathophysiology. Moreover, it has been reported that activation of CaMKII via the $G_{q}$ signaling pathway contributed to this hypertrophic growth and gene expression (Anderson et al., 2011; Colomer et al., 2003). Upon activation, CaMKII can increase histone deacetylase (HDAC) phosphorylation and nuclear export, which results in myocyte enhancer factor 2driven transcriptions. Therefore, alter the expression of a range of hypertrophyassociated genes, which can be prevented by the inhibition of CaMKII (Ramirez et al., 1997; Sei et al., 1991; Zhu et al., 2000). Results from this study show that CaMKII expression and phosphorylation both get increased upon chronic AngII treatment and pressure overload. Thus, may contribute equally to both WT and KI mice. However, the difference in the expression of catalytic subunit along with oxidative PKA activation may be the ultimate determinate for hypertrophy. Because in AngII model, WT animal show hypertrophy as expected, but KI mice with its similar PKA catalytic subunit (PKA C) expression to WT animals, could produce a comparable level of hypertrophy. This may help the mice to cope the 
stress. Thus KI can show similar in vivo contractility. In contrast, upon pressure overload, WT animals had much-controlled hypertrophy because it has sufficient PKA C, as same as control mice and also has oxidative PKA activation pathway. KI animals, however, with their less PKA C and inability to activate PKA oxidatively, showed a severe hypertrophic response to compensate contractile dysfunction. Supporting our findings, Ha et al. described a PKA-mediated mechanism regulating cardiac hypertrophy, where they have shown that PKA-dependent phosphorylation of HDAC5 at S280, inhibit its nuclear export leading to inhibition of myocyte enhancer factor 2 (MEF2) mediated fetal gene expression and hypertrophy (Ha et al., 2010). Thus, in WT mice CaMKII-mediated hypertrophy is counterbalanced via PKA, which is not possible in KI animals because of their inability to activate redox-active PKA.

\subsection{Pressure overload increases arrhythmogenic propensity in KI mice}

Cardiac arrhythmias are instigated due to atypical rate and rhythm of the heartbeat caused by disturbed electrophysiology of the myocardium (Youn et al., 2013). Interestingly, anti-arrhythmic drugs including $\beta$-blockers, Amiodarone, Dronedarone, Dofetilide, and Sotalol have been widely used for the treatment of atrial fibrillation (AF) by blocking $\beta$-adrenergic receptors or ion channels (Patel et al., 2011). Although therapy is beneficial, the absence of long-term efficacy, offtarget side effects, or drug-induced pro-arrhythmic effects has been repeatedly reported for these drugs (Sanguinetti and Bennett, 2003). In addition to $\beta$ receptor dysregulation, both loss and gain of function mutations of LTCC have been reported to be involved in ventricular arrhythmia (Burashnikov et al., 2010; Splawski et al., 2005). As found in this study, decreased $\mathrm{I}_{\mathrm{Ca}}$ current in $\mathrm{KI}$ mice, upon AngII and pressure overload-induced stress, may also be proarrhythmogenic. This finding is further supported by Koncz et al. who reported that early repolarization (ER) due to decrease in $I_{C a}$ could induce a severe arrhythmia in an animal model of ER syndrome (Koncz et al., 2014).

Moreover, increased levels of ROS such as superoxide and $\mathrm{H}_{2} \mathrm{O}_{2}$ have been found to be associated with AF (Zhang et al., 2012). It has been reported that either ex- 
ogenously applied ROS $\left(\mathrm{H}_{2} \mathrm{O}_{2}\right)$ or endogenously generated ROS by AngII stimulation can induce ventricular arrhythmias via the CaMKII pathway. Treatment with the CaMKII inhibitor KN-93 attenuated the occurrence of AngII-induced EADs (Xie et al., 2009; Zhao et al., 2011). Pereira et al. showed that $\beta_{1}$-AR activates Epac2 to induce SR Ca ${ }^{2+}$ leak via $\mathrm{CaMKII}_{\delta}$-dependent phosphorylation of RyR2S2814, which could induce arrhythmia; in contrast to $\beta_{2}$, where LTCC and PKA localize (Pereira et al., 2013). Moreover, a rabbit model of HF by aortic insufficiency followed by aortic constriction, SR calcium leak, and arrhythmia was prevented using CaMKII inhibitor but not by PKA inhibitors (Ai et al., 2005). Excitingly, in vivo data of this study show that even though AngII and pressure overload increased CaMKII expression and auto-phosphorylation in both WT and KI animals, EPU can only induce an arrhythmia in KI mice after six weeks of TAC. Leroy et al. showed a similar result where burst pacing could not induce an arrhythmia in WT animals even after a high dose of isoprenaline injection (Leroy et al., 2011). Prolonged QTc and TT interval of KI mice, which may involve in shortening of the action potential (Laurita and Katra, 2005), supports the finding that KI mice have more propensity to arrhythmia after TAC than WT and shamoperated mice. Moreover, expression of the catalytic subunit of PKA, which was decreased upon pressure overload and mildly decreased upon AngII, additionally supports the idea that reduced PKA activity is associated with increased arrhythmia in KI mice. In summary, CaMKII-mediated detrimental functions are opposed by PKA. That's why KI with its similar PKA C can cope against arrhythmia upon AngII exposure. However, in pressure overload, reduction in PKA C, as well as the inability to oxidative PKA activation, cannot protect KI mice against arrhythmia. Therefore, CaMKII may potentiate arrhythmogenic behavior only if the beneficial role of PKA is absent or reduced in these settings.

\subsection{KI animals exhibit higher mortality rate upon TAC operation}

Cardiac hypertrophy is one of the physiological adaptive responses of the heart to volume or pressure overload. It involves, for example, cardiomyocyte enlargement, cardiac fibroblast proliferation and collagen formation, extensive remodeling of ion channels, gap junctions, calcium handling, and the cytoskeleton. Nonetheless, persistent hypertrophy due to chronic hypertension, valvular dis- 
eases or aortic stenosis consequently reduce the excitability of cardiac myocytes, induce arrhythmias and eventually cause heart failure (Kahan and Bergfeldt, 2005). These findings are in agreement with this study where all these contractile abnormalities, hypertrophy, and increased arrhythmogenic propensity make KI mice more prone to heart failure compared to WT littermates. It is found that KI died significantly earlier than WT littermates after six weeks of TAC. Interestingly, heterozygous C17S mice show longer survival than KI but shorter than WT TAC mice. $I_{\mathrm{Ca}}$ data show a similar intermediate pattern. Thus, it can well be that redox-active PKA type I modulates LTCC function and provides a mechanism to cope with the stress conditions to WT and Het mice, which is absent in KI animals. However, no death of WT or KI mice were recorded in AngII infusion model, implying that KI animals can survive mild stress upon changing different contractile parameters, which is not possible under extreme stress condition.

\subsection{Diseased patients show oxidative activation of PKA}

It has been well documented that oxidative stress is associated with disease conditions (Bers, 2001). This increase in oxidants may activate oxidative PKA signaling pathway. Human atrial samples from atrial fibrillation (AF) and obstructive sleep apnea (OSA) patients show analogous results, where permanent AF and severe OSA patients (apnea-hypopnea index, AHI>10) show a significant increase in PKA dimer formation compared to their sinus rhythm counterparts. Interestingly, mild AF patients (paroxysmal AF) or mild OSA $(\mathrm{AHI}<10)$ show a comparable amount of dimer as like sinus rhythm patients. It may well be that at extremely diseased conditions produce more ROS to initiate special signaling, like oxidative PKA signaling pathway, to protect the heart. However, when this fails, organ damage happens.

\subsection{Failure of antioxidant trials in providing protection against CVD}

Although oxidative stress is implicated as a causative agent for cardiovascular diseases, most of the large clinical trials of antioxidant failed to show any significant protection against cardiovascular diseases (1999; de Gaetano and 
Collaborative Group of the Primary Prevention, 2001; Greenberg et al., 1996; Heart Protection Study Collaborative, 2002; Hennekens et al., 1996; Hodis et al., 2002; Lonn et al., 2002; Virtamo et al., 1998; Yusuf et al., 2000). For example, application of vitamin E, beta-carotene or antioxidant cocktails could not give any advantage to patients with different heart diseases, like myocardial infarction (MI), stroke or CVD associated death. Moreover, few clinical studies even showed severe adverse effects such as death upon the antioxidant therapy. As found in ATBC (deal with nonsmokers) or CARET (deal with Asbestos workers) study, where antioxidant therapy induces a severe hemorrhagic stroke or all-cause mortality, respectively (1994; Brown et al., 2001; Omenn et al., 1996; Waters et al., 2002). Although few studies showed beneficial effects of antioxidant treatment (Boaz et al., 2000; Rapola et al., 1997; Salonen et al., 2000; Stephens et al., 1996), it is still in doubt that whether antioxidant could be an option for therapy. In addition to these human trial data, a recent report in a mouse model showed similar results, where cardiac-specific overexpression of an antioxidant protein, Hsp27, increased cardiomyopathy significantly compared to control (Zhang et al., 2010). All these data strengthened the findings of this study that in addition to having beneficial roles, antioxidants might have some adverse effects on the physiologic system as well, and in our hand, this may be due to inhibition of oxidative activation of PKA and other proteins along with their downstream signaling pathways.

\subsection{Conclusion}

Catecholamine-mediated cardiac inotropic, chronotropic, and lusitropic effects are mostly maintained by $\beta$-AR/PKA-cAMP signaling pathway. Many reports showed alteration of this signaling pathway during various heart diseases including heart failure (Bristow et al., 1982; Ungerer et al., 1993). The severity of the disease depends on the degree of receptor down regulation and the functional loss of the remaining receptors (Brodde et al., 1989; Engelhardt et al., 1996). It has been proposed that restoration of $\beta$-adrenergic receptor responsiveness or PKA activity might be a strategy to improve cardiac function (Zakhary et al., 1999). Interestingly, overexpression of either the catalytic subunit of PKA (Antos et al., 2001) or $\beta_{1}$-receptor (Bisognano et al., 2000; Engelhardt et al., 1999) or 
$\mathrm{G}_{\alpha \mathrm{s}}$-overexpression (Iwase et al., 1996) in animal models showed cardiomyocyte hypertrophy, fibrosis, and a progressive decline in cardiac function resulting ultimate death from cardiac failure; suggesting detrimental role of $\beta$-AR/PKAcAMP signaling pathway. It may well be that overexpression of PKA catalytic subunit or $\beta_{1}$ or $G_{\alpha s}$ may not be tolerable for the cell. Because overexpression models are not entirely physiologic, as overexpressed protein might have altered localization and interaction with partner proteins, which can also influence the deleterious outcome for the heart. It may also elicit an excessive response, even at physiologic catecholamine concentration, which can damage the cell (Lohse and Engelhardt, 2001).

In this study, I report that under such stressed conditions, oxidant could modulate the $\beta$-AR/PKA-cAMP signaling pathway via oxidative activation of PKA. Upon stress condition, excess oxidant activates PKA and phosphorylates downstream targets specially LTCC, to maintain inotropic effects. Oxidative PKA activation is necessary for this stress condition because $\beta$-AR receptors and its downstream effector cAMP are down regulated upon stress, thus oxidized PKA can modulate cellular function without the need of receptors and compensate in this setting to maintain contraction. This result is in agreement with the findings that, mice that are lacking $\beta$-adrenergic receptors have a substantially normal basal cardiac function (Rohrer et al., 1996), which could be mediated by this basal oxidative PKA activation, as also mentioned in this study. Similarly, it has been found that overexpression of adenylyl cyclase in the heart results in enhancement of adrenergically stimulated cAMP levels, which could improve cardiac function (Gao et al., 1999; Roth et al., 1999), thus implicating the supportive role of cAMP and oxidants to each other.

Another point is that, $\beta_{1}$ and $\beta_{2}$ receptors maintain their own compartment of cAMP in the cell (Di Benedetto et al., 2008). LTCC has been reported to be mostly located in $\beta_{2}$ compartments, which is concentrated on T-tubules. Interestingly, $\beta$ adrenergic signaling activated more $\mathrm{I}_{\mathrm{Ca}}$ response in T-tubules than in surface membrane, where $\beta_{1}$ located (Chase et al., 2010). Thus, overexpression of $\beta_{1}$ may be unable to produce sufficient cAMP to activate PKA over another compartment. 
On the other side, overexpression of $\beta_{2}$-adrenergic receptors is beneficial for the cell similar to adenylyl cyclase overexpression. The reason is that both these pathways may produce enough cAMP to activate PKA and LTCC (Liggett et al., 2000; Milano et al., 1994). In this scenario, it is also mentionable that PKA type I is well distributed and freely migrating in the cytoplasm (Cummings et al., 1996). This could also explain another beneficial role of PKA, by which it can get activated in a different compartment, may be via oxidation and activate proteins in other compartments if needed. This type of situation may arise in heart failure, where T-tubule numbers get decreased but oxidants get increased (Nikolaev et al., 2010). Thus, PKA may switch from its classical activation to oxidative activation pathway.

Nonetheless, like the overexpression of $\beta_{1}$ or $G_{\alpha s}$, excess oxidation of PKA may be detrimental for the cell. This idea can explain the heart failure phenotype where excess oxidant cannot contribute to inotropic effects rather cause damage to the cell. WT pressure overloaded mice are an example in this setting, where they showed a significant increase in survival compared to KI, but not $100 \%$ rescue of the heart failure phenotype. 


\section{References}


1. (1994). The effect of vitamin $\mathrm{E}$ and beta carotene on the incidence of lung cancer and other cancers in male smokers. The Alpha-Tocopherol, Beta Carotene Cancer Prevention Study Group. The New England journal of medicine 330, 1029-1035.

2. (1999). Dietary supplementation with $\mathrm{n}-3$ polyunsaturated fatty acids and vitamin $\mathrm{E}$ after myocardial infarction: results of the GISSI-Prevenzione trial. Gruppo Italiano per lo Studio della Sopravvivenza nell'Infarto miocardico. Lancet 354, 447-455.

3. Abramson, J.J., and Salama, G. (1989). Critical sulfhydryls regulate calcium release from sarcoplasmic reticulum. J Bioenerg Biomembr 21, 283-294.

4. Ago, T., Kitazono, T., Ooboshi, H., Iyama, T., Han, Y.H., Takada, J., Wakisaka, M., Ibayashi, S., Utsumi, H., and Iida, M. (2004). Nox4 as the major catalytic component of an endothelial NAD(P)H oxidase. Circulation 109, 227-233.

5. Ai, X., Curran, J.W., Shannon, T.R., Bers, D.M., and Pogwizd, S.M. (2005). $\mathrm{Ca} 2+/$ calmodulin-dependent protein kinase modulates cardiac ryanodine receptor phosphorylation and sarcoplasmic reticulum Ca2+ leak in heart failure. Circ Res 97, 1314-1322.

6. Aiello, E.A., and Cingolani, H.E. (2001). Angiotensin II stimulates cardiac L-type $\mathrm{Ca}(2+)$ current by a $\mathrm{Ca}(2+)$ - and protein kinase C-dependent mechanism. Am J Physiol Heart Circ Physiol 280, H1528-1536.

7. Aller, I., Rouhier, N., and Meyer, A.J. (2013). Development of roGFP2-derived redox probes for measurement of the glutathione redox potential in the cytosol of severely glutathionedeficient rml1 seedlings. Frontiers in plant science 4, 506.

8. Ambrosio, G., Becker, L.C., Hutchins, G.M., Weisman, H.F., and Weisfeldt, M.L. (1986). Reduction in experimental infarct size by recombinant human superoxide dismutase: insights into the pathophysiology of reperfusion injury. Circulation 74, 1424-1433.

9. Amieux, P.S., and McKnight, G.S. (2002). The essential role of RI alpha in the maintenance of regulated PKA activity. Annals of the New York Academy of Sciences 968, 75-95.

10. An, M., and Zamponi, G. (2000-2013). Voltage-Dependent Inactivation of Voltage Gated Calcium Channels (Austin (TX): Landes Bioscience).

11. Anderson, M.E., Brown, J.H., and Bers, D.M. (2011). CaMKII in myocardial hypertrophy and heart failure. J Mol Cell Cardiol 51, 468-473.

12. Andersson, D.C., Fauconnier, J., Yamada, T., Lacampagne, A., Zhang, S.J., Katz, A., and Westerblad, H. (2011). Mitochondrial production of reactive oxygen species contributes to the beta-adrenergic stimulation of mouse cardiomycytes. The Journal of physiology 589, 1791-1801.

13. Antos, C.L., Frey, N., Marx, S.O., Reiken, S., Gaburjakova, M., Richardson, J.A., Marks, A.R., and Olson, E.N. (2001). Dilated cardiomyopathy and sudden death resulting from constitutive activation of protein kinase a. Circ Res 89, 997-1004.

14. Anversa, P., and Kajstura, J. (1998). Ventricular myocytes are not terminally differentiated in the adult mammalian heart. Circ Res 83, 1-14.

15. Anversa, P., Kajstura, J., and Olivetti, G. (1996). Myocyte death in heart failure. Current opinion in cardiology 11, 245-251.

16. Anzai, K., Ogawa, K., Kuniyasu, A., Ozawa, T., Yamamoto, H., and Nakayama, H. (1998). Effects of hydroxyl radical and sulfhydryl reagents on the open probability of the purified cardiac ryanodine receptor channel incorporated into planar lipid bilayers. Biochem Biophys Res Commun 249, 938-942.

17. Baker, K.M., Chernin, M.I., Wixson, S.K., and Aceto, J.F. (1990). Renin-angiotensin system involvement in pressure-overload cardiac hypertrophy in rats. The American journal of physiology 259, H324-332.

18. Beebe, S.J., Oyen, O., Sandberg, M., Froysa, A., Hansson, V., and Jahnsen, T. (1990). Molecular cloning of a tissue-specific protein kinase (C gamma) from human testis--representing a third isoform for the catalytic subunit of cAMP-dependent protein kinase. Molecular endocrinology 4, 465-475. 
19. Belevych, A.E., Terentyev, D., Terentyeva, R., Ho, H.T., Gyorke, I., Bonilla, I.M., Carnes, C.A., Billman, G.E., and Gyorke, S. (2012). Shortened Ca2+ signaling refractoriness underlies cellular arrhythmogenesis in a postinfarction model of sudden cardiac death. Circ Res 110, 569-577.

20. Bendall, J.K., Cave, A.C., Heymes, C., Gall, N., and Shah, A.M. (2002). Pivotal role of a gp91(phox)-containing NADPH oxidase in angiotensin II-induced cardiac hypertrophy in mice. Circulation 105, 293-296.

21. Berjukow, S., Marksteiner, R., Sokolov, S., Weiss, R.G., Margreiter, E., and Hering, S. (2001). Amino acids in segment IVS6 and beta-subunit interaction support distinct conformational changes during Ca(v)2.1 inactivation. J Biol Chem 276, 17076-17082.

22. Berry, C.E., and Hare, J.M. (2004). Xanthine oxidoreductase and cardiovascular disease: molecular mechanisms and pathophysiological implications. The Journal of physiology 555, 589606.

23. Bers, D.M. (2001). Excitation-Contraction Coupling and Cardiac Contractile Force, Vol 237 (Springer Netherlands).

24. Bers, D.M. (2002). Cardiac excitation-contraction coupling. Nature 415, 198-205.

25. Bers, D.M. (2008). Calcium cycling and signaling in cardiac myocytes. Annual review of physiology 70, 23-49.

26. Bers, D.M., Eisner, D.A., and Valdivia, H.H. (2003). Sarcoplasmic reticulum Ca2+ and heart failure: roles of diastolic leak and Ca2+ transport. Circ Res 93, 487-490.

27. Bers, D.M., and Guo, T. (2005). Calcium signaling in cardiac ventricular myocytes. Annals of the New York Academy of Sciences 1047, 86-98.

28. Beuckelmann, D.J., Nabauer, M., and Erdmann, E. (1992). Intracellular calcium handling in isolated ventricular myocytes from patients with terminal heart failure. Circulation 85, 10461055.

29. Bishop, J.E. (1998). Regulation of cardiovascular collagen deposition by mechanical forces. Molecular medicine today 4, 69-75.

30. Bisognano, J.D., Weinberger, H.D., Bohlmeyer, T.J., Pende, A., Raynolds, M.V., Sastravaha, A., Roden, R., Asano, K., Blaxall, B.C., Wu, S.C., et al. (2000). Myocardial-directed overexpression of the human beta(1)-adrenergic receptor in transgenic mice. J Mol Cell Cardiol 32, 817-830.

31. Bjelakovic, G., Nikolova, D., Gluud, L.L., Simonetti, R.G., and Gluud, C. (2007). Mortality in randomized trials of antioxidant supplements for primary and secondary prevention: systematic review and meta-analysis. Jama 297, 842-857.

32. Boaz, M., Smetana, S., Weinstein, T., Matas, Z., Gafter, U., Iaina, A., Knecht, A., Weissgarten, Y., Brunner, D., Fainaru, M., et al. (2000). Secondary prevention with antioxidants of cardiovascular disease in endstage renal disease (SPACE): randomised placebo-controlled trial. Lancet 356, 1213-1218.

33. Bodi, I., Mikala, G., Koch, S.E., Akhter, S.A., and Schwartz, A. (2005). The L-type calcium channel in the heart: the beat goes on. The Journal of clinical investigation 115, 3306-3317.

34. Bohm, M., and Lohse, M.J. (1994). Quantification of beta-adrenoceptors and betaadrenoceptor kinase on protein and mRNA levels in heart failure. European heart journal 15 Suppl D, 30-34.

35. Boraso, A., and Williams, A.J. (1994). Modification of the gating of the cardiac sarcoplasmic reticulum $\mathrm{Ca}(2+)$-release channel by $\mathrm{H} 2 \mathrm{O} 2$ and dithiothreitol. The American journal of physiology 267, H1010-1016.

36. Brady, N.R., Hamacher-Brady, A., Westerhoff, H.V., and Gottlieb, R.A. (2006). A wave of reactive oxygen species (ROS)-induced ROS release in a sea of excitable mitochondria. Antioxidants \& redox signaling 8, 1651-1665.

37. Brandes, R.P. (2005). Triggering mitochondrial radical release: a new function for NADPH oxidases. Hypertension 45, 847-848. 
38. Brandes, R.P., and Kreuzer, J. (2005). Vascular NADPH oxidases: molecular mechanisms of activation. Cardiovasc Res 65, 16-27.

39. Brennan, J.P., Bardswell, S.C., Burgoyne, J.R., Fuller, W., Schroder, E., Wait, R., Begum, S., Kentish, J.C., and Eaton, P. (2006). Oxidant-induced activation of type I protein kinase A is mediated by RI subunit interprotein disulfide bond formation. J Biol Chem 281, 21827-21836.

40. Brilla, C.G., Pick, R., Tan, L.B., Janicki, J.S., and Weber, K.T. (1990). Remodeling of the rat right and left ventricles in experimental hypertension. Circ Res 67, 1355-1364.

41. Bristow, M.R., Ginsburg, R., Minobe, W., Cubicciotti, R.S., Sageman, W.S., Lurie, K., Billingham, M.E., Harrison, D.C., and Stinson, E.B. (1982). Decreased catecholamine sensitivity and beta-adrenergic-receptor density in failing human hearts. The New England journal of medicine 307, 205-211.

42. Brodde, O.E., Zerkowski, H.R., Doetsch, N., Motomura, S., Khamssi, M., and Michel, M.C. (1989). Myocardial beta-adrenoceptor changes in heart failure: concomitant reduction in beta 1and beta 2-adrenoceptor function related to the degree of heart failure in patients with mitral valve disease. J Am Coll Cardiol 14, 323-331.

43. Brown, B.G., Zhao, X.Q., Chait, A., Fisher, L.D., Cheung, M.C., Morse, J.S., Dowdy, A.A., Marino, E.K., Bolson, E.L., Alaupovic, P., et al. (2001). Simvastatin and niacin, antioxidant vitamins, or the combination for the prevention of coronary disease. The New England journal of medicine $345,1583-1592$.

44. Brown, H.F., Kimura, J., Noble, D., Noble, S.J., and Taupignon, A. (1984). The slow inward current, isi, in the rabbit sino-atrial node investigated by voltage clamp and computer simulation. Proceedings of the Royal Society of London Series B, Biological sciences 222, 305-328.

45. Bryant, S., Kimura, T.E., Kong, C.H., Watson, J.J., Chase, A., Suleiman, M.S., James, A.F., and Orchard, C.H. (2014). Stimulation of ICa by basal PKA activity is facilitated by caveolin-3 in cardiac ventricular myocytes. J Mol Cell Cardiol 68, 47-55.

46. Bunemann, M., Lee, K.B., Pals-Rylaarsdam, R., Roseberry, A.G., and Hosey, M.M. (1999). Desensitization of G-protein-coupled receptors in the cardiovascular system. Annual review of physiology 61, 169-192.

47. Burashnikov, E., Pfeiffer, R., Barajas-Martinez, H., Delpon, E., Hu, D., Desai, M., Borggrefe, M., Haissaguerre, M., Kanter, R., Pollevick, G.D., et al. (2010). Mutations in the cardiac L-type calcium channel associated with inherited J-wave syndromes and sudden cardiac death. Heart rhythm : the official journal of the Heart Rhythm Society 7, 1872-1882.

48. Burdon, R.H. (1995). Superoxide and hydrogen peroxide in relation to mammalian cell proliferation. Free radical biology \& medicine 18, 775-794.

49. Burgoyne, J.R., and Eaton, P. (2009). Transnitrosylating nitric oxide species directly activate type I protein kinase A, providing a novel adenylate cyclase-independent cross-talk to beta-adrenergic-like signaling. J Biol Chem 284, 29260-29268.

50. Burgoyne, J.R., Mongue-Din, H., Eaton, P., and Shah, A.M. (2012). Redox signaling in cardiac physiology and pathology. Circ Res 111, 1091-1106.

51. Burgoyne, J.R., Rudyk, O., Cho, H.J., Prysyazhna, O., Hathaway, N., Weeks, A., Evans, R., Ng, T., Schroder, K., Brandes, R.P., et al. (2015). Deficient angiogenesis in redox-dead Cys17Ser PKARIalpha knock-in mice. Nature communications 6, 7920.

52. Burton, K.A., Johnson, B.D., Hausken, Z.E., Westenbroek, R.E., Idzerda, R.L., Scheuer, T., Scott, J.D., Catterall, W.A., and McKnight, G.S. (1997). Type II regulatory subunits are not required for the anchoring-dependent modulation of Ca2+ channel activity by cAMP-dependent protein kinase. Proceedings of the National Academy of Sciences of the United States of America 94, 11067-11072.

53. Cai, H., Davis, M.E., Drummond, G.R., and Harrison, D.G. (2001). Induction of endothelial NO synthase by hydrogen peroxide via a $\mathrm{Ca}(2+) /$ calmodulin-dependent protein kinase II/janus kinase 2-dependent pathway. Arteriosclerosis, thrombosis, and vascular biology 21, 1571-1576. 
54. Campbell, D.J. (1987). Circulating and tissue angiotensin systems. The Journal of clinical investigation 79, 1-6.

55. Cappola, T.P., Kass, D.A., Nelson, G.S., Berger, R.D., Rosas, G.O., Kobeissi, Z.A., Marban, E., and Hare, J.M. (2001). Allopurinol improves myocardial efficiency in patients with idiopathic dilated cardiomyopathy. Circulation 104, 2407-2411.

56. Carmichael, D.F., Geahlen, R.L., Allen, S.M., and Krebs, E.G. (1982). Type II regulatory subunit of cAMP-dependent protein kinase. Phosphorylation by casein kinase II at a site that is also phosphorylated in vivo. J Biol Chem 257, 10440-10445.

57. Chan, E.D., Riches, D.W., and White, C.W. (2001). Redox paradox: effect of Nacetylcysteine and serum on oxidation reduction-sensitive mitogen-activated protein kinase signaling pathways. American journal of respiratory cell and molecular biology 24, 627-632.

58. Chase, A., Colyer, J., and Orchard, C.H. (2010). Localised Ca channel phosphorylation modulates the distribution of L-type Ca current in cardiac myocytes. J Mol Cell Cardiol 49, 121131.

59. Chaves, M.M., Costa, D.C., de Oliveira, B.F., Rocha, M.I., and Nogueira-Machado, J.A. (2009). Role PKA and p38 MAPK on ROS production in neutrophil age-related: Lack of IL-10 effect in older subjects. Mechanisms of ageing and development 130, 588-591.

60. Cho, J., Won, K., Wu, D., Soong, Y., Liu, S., Szeto, H.H., and Hong, M.K. (2007). Potent mitochondria-targeted peptides reduce myocardial infarction in rats. Coronary artery disease 18 , 215-220.

61. Choi, D.W. (1988). Calcium-mediated neurotoxicity: relationship to specific channel types and role in ischemic damage. Trends in neurosciences 11, 465-469.

62. Chow, C.K., Ibrahim, W., Wei, Z., and Chan, A.C. (1999). Vitamin E regulates mitochondrial hydrogen peroxide generation. Free radical biology \& medicine 27, 580-587.

63. Clegg, C.H., Ran, W., Uhler, M.D., and McKnight, G.S. (1989). A mutation in the catalytic subunit of protein kinase A prevents myristylation but does not inhibit biological activity. J Biol Chem 264, 20140-20146.

64. Cohen, N.M., and Lederer, W.J. (1987). Calcium current in isolated neonatal rat ventricular myocytes. The Journal of physiology 391, 169-191.

65. Colomer, J.M., Mao, L., Rockman, H.A., and Means, A.R. (2003). Pressure overload selectively up-regulates $\mathrm{Ca} 2+$ /calmodulin-dependent protein kinase II in vivo. Molecular endocrinology 17, 183-192.

66. Colucci, W.S. (1997). Molecular and cellular mechanisms of myocardial failure. The American journal of cardiology 80, 15L-25L.

67. Corbin, J.D., Sugden, P.H., Lincoln, T.M., and Keely, S.L. (1977). Compartmentalization of adenosine $3^{\prime}: 5$ '-monophosphate and adenosine 3 ':5'-monophosphate-dependent protein kinase in heart tissue. J Biol Chem 252, 3854-3861.

68. Cummings, D.E., Brandon, E.P., Planas, J.V., Motamed, K., Idzerda, R.L., and McKnight, G.S. (1996). Genetically lean mice result from targeted disruption of the RII beta subunit of protein kinase A. Nature 382, 622-626.

69. Dai, D.F., Chen, T., Szeto, H., Nieves-Cintron, M., Kutyavin, V., Santana, L.F., and Rabinovitch, P.S. (2011a). Mitochondrial targeted antioxidant Peptide ameliorates hypertensive cardiomyopathy. J Am Coll Cardiol 58, 73-82.

70. Dai, D.F., Hsieh, E.J., Chen, T., Menendez, L.G., Basisty, N.B., Tsai, L., Beyer, R.P., Crispin, D.A., Shulman, N.J., Szeto, H.H., et al. (2013). Global proteomics and pathway analysis of pressureoverload-induced heart failure and its attenuation by mitochondrial-targeted peptides. Circulation Heart failure 6, 1067-1076.

71. Dai, D.F., Johnson, S.C., Villarin, J.J., Chin, M.T., Nieves-Cintron, M., Chen, T., Marcinek, D.J., Dorn, G.W., 2nd, Kang, Y.J., Prolla, T.A., et al. (2011b). Mitochondrial oxidative stress mediates 
angiotensin II-induced cardiac hypertrophy and Galphaq overexpression-induced heart failure. Circ Res 108, 837-846.

72. Daiber, A. (2010). Redox signaling (cross-talk) from and to mitochondria involves mitochondrial pores and reactive oxygen species. Biochimica et biophysica acta 1797, 897-906.

73. Davis, B.A., Schwartz, A., Samaha, F.J., and Kranias, E.G. (1983). Regulation of cardiac sarcoplasmic reticulum calcium transport by calcium-calmodulin-dependent phosphorylation. J Biol Chem 258, 13587-13591.

74. de Gaetano, G., and Collaborative Group of the Primary Prevention, P. (2001). Low-dose aspirin and vitamin $\mathrm{E}$ in people at cardiovascular risk: a randomised trial in general practice. Collaborative Group of the Primary Prevention Project. Lancet 357, 89-95.

75. De Jongh, K.S., Murphy, B.J., Colvin, A.A., Hell, J.W., Takahashi, M., and Catterall, W.A. (1996). Specific phosphorylation of a site in the full-length form of the alpha 1 subunit of the cardiac L-type calcium channel by adenosine $3^{\prime}, 5^{\prime}$-cyclic monophosphate-dependent protein kinase. Biochemistry 35, 10392-10402.

76. Desseyn, J.L., Burton, K.A., and McKnight, G.S. (2000). Expression of a nonmyristylated variant of the catalytic subunit of protein kinase A during male germ-cell development. Proceedings of the National Academy of Sciences of the United States of America 97, 6433-6438.

77. Di Benedetto, G., Zoccarato, A., Lissandron, V., Terrin, A., Li, X., Houslay, M.D., Baillie, G.S., and Zaccolo, M. (2008). Protein kinase A type I and type II define distinct intracellular signaling compartments. Circ Res 103, 836-844.

78. Dikalov, S.I., and Nazarewicz, R.R. (2013). Angiotensin II-induced production of mitochondrial reactive oxygen species: potential mechanisms and relevance for cardiovascular disease. Antioxidants \& redox signaling 19, 1085-1094.

79. Dipla, K., Mattiello, J.A., Margulies, K.B., Jeevanandam, V., and Houser, S.R. (1999). The sarcoplasmic reticulum and the $\mathrm{Na}+\mathrm{Ca} 2+$ exchanger both contribute to the $\mathrm{Ca} 2+$ transient of failing human ventricular myocytes. Circ Res 84, 435-444.

80. Diskar, M., Zenn, H.M., Kaupisch, A., Kaufholz, M., Brockmeyer, S., Sohmen, D., Berrera, M., Zaccolo, M., Boshart, M., Herberg, F.W., et al. (2010). Regulation of cAMP-dependent protein kinases: the human protein kinase $\mathrm{X}$ ( $\mathrm{PrKX})$ reveals the role of the catalytic subunit alphaHalphaI loop. J Biol Chem 285, 35910-35918.

81. Dixon, I.M., Hata, T., and Dhalla, N.S. (1992). Sarcolemmal Na(+)-K(+)-ATPase activity in congestive heart failure due to myocardial infarction. The American journal of physiology 262, C664-671.

82. Doerries, C., Grote, K., Hilfiker-Kleiner, D., Luchtefeld, M., Schaefer, A., Holland, S.M., Sorrentino, S., Manes, C., Schieffer, B., Drexler, H., et al. (2007). Critical role of the NAD(P)H oxidase subunit p47phox for left ventricular remodeling/dysfunction and survival after myocardial infarction. Circ Res 100, 894-903.

83. Donath, S., Li, P., Willenbockel, C., Al-Saadi, N., Gross, V., Willnow, T., Bader, M., Martin, U., Bauersachs, J., Wollert, K.C., et al. (2006). Apoptosis repressor with caspase recruitment domain is required for cardioprotection in response to biomechanical and ischemic stress. Circulation $113,1203-1212$.

84. Dorn, G.W., 2nd (2009). Apoptotic and non-apoptotic programmed cardiomyocyte death in ventricular remodelling. Cardiovasc Res 81, 465-473.

85. Dorn, G.W., 2nd, and Force, T. (2005). Protein kinase cascades in the regulation of cardiac hypertrophy. The Journal of clinical investigation 115, 527-537.

86. Dostal, D.E., Rothblum, K.N., Chernin, M.I., Cooper, G.R., and Baker, K.M. (1992). Intracardiac detection of angiotensinogen and renin: a localized renin-angiotensin system in neonatal rat heart. The American journal of physiology 263, C838-850.

87. Drake-Holland, A.J., Noble, M.I., and Lab, M.J. (2001). Acute pressure overload cardiac arrhythmias are dependent on the presence of myocardial tissue catecholamines. Heart 85, 576. 
88. Durell, S.R., Hao, Y., and Guy, H.R. (1998). Structural models of the transmembrane region of voltage-gated and other $\mathrm{K}+$ channels in open, closed, and inactivated conformations. Journal of structural biology 121, 263-284.

89. Eager, K.R., and Dulhunty, A.F. (1998). Activation of the cardiac ryanodine receptor by sulfhydryl oxidation is modified by Mg2+ and ATP. The Journal of membrane biology 163, 9-18.

90. Ekelund, U.E., Harrison, R.W., Shokek, O., Thakkar, R.N., Tunin, R.S., Senzaki, H., Kass, D.A., Marban, E., and Hare, J.M. (1999). Intravenous allopurinol decreases myocardial oxygen consumption and increases mechanical efficiency in dogs with pacing-induced heart failure. Circ Res 85, 437-445.

91. Engelhardt, S., Bohm, M., Erdmann, E., and Lohse, M.J. (1996). Analysis of beta-adrenergic receptor mRNA levels in human ventricular biopsy specimens by quantitative polymerase chain reactions: progressive reduction of beta 1-adrenergic receptor mRNA in heart failure. J Am Coll Cardiol 27, 146-154.

92. Engelhardt, S., Hein, L., Wiesmann, F., and Lohse, M.J. (1999). Progressive hypertrophy and heart failure in beta1-adrenergic receptor transgenic mice. Proceedings of the National Academy of Sciences of the United States of America 96, 7059-7064.

93. Erickson, J.R., Joiner, M.L., Guan, X., Kutschke, W., Yang, J., Oddis, C.V., Bartlett, R.K., Lowe, J.S., O'Donnell, S.E., Aykin-Burns, N., et al. (2008). A dynamic pathway for calcium-independent activation of CaMKII by methionine oxidation. Cell 133, 462-474.

94. Eschenhagen, T. (2010). Is ryanodine receptor phosphorylation key to the fight or flight response and heart failure? The Journal of clinical investigation 120, 4197-4203.

95. Eu, J.P., Sun, J., Xu, L., Stamler, J.S., and Meissner, G. (2000). The skeletal muscle calcium release channel: coupled 02 sensor and NO signaling functions. Cell 102, 499-509.

96. Fan, D., Wannenburg, T., and de Tombe, P.P. (1997). Decreased myocyte tension development and calcium responsiveness in rat right ventricular pressure overload. Circulation 95, 2312-2317.

97. Faraci, F.M., and Didion, S.P. (2004). Vascular protection: superoxide dismutase isoforms in the vessel wall. Arteriosclerosis, thrombosis, and vascular biology 24, 1367-1373.

98. Favero, T.G., Zable, A.C., and Abramson, J.J. (1995). Hydrogen peroxide stimulates the $\mathrm{Ca} 2+$ release channel from skeletal muscle sarcoplasmic reticulum. J Biol Chem 270, 2555725563.

99. Fearon, I.M., Palmer, A.C., Balmforth, A.J., Ball, S.G., Varadi, G., and Peers, C. (1999). Modulation of recombinant human cardiac L-type $\mathrm{Ca} 2+$ channel alpha1C subunits by redox agents and hypoxia. The Journal of physiology 514 ( Pt 3), 629-637.

100. Feldman, A.M., Weinberg, E.O., Ray, P.E., and Lorell, B.H. (1993). Selective changes in cardiac gene expression during compensated hypertrophy and the transition to cardiac decompensation in rats with chronic aortic banding. Circ Res 73, 184-192.

101. Fill, M., and Copello, J.A. (2002). Ryanodine receptor calcium release channels. Physiol Rev 82, 893-922.

102. Fitzpatrick, D.B., and Karmazyn, M. (1984). Comparative effects of calcium channel blocking agents and varying extracellular calcium concentration on hypoxia/reoxygenation and ischemia/reperfusion-induced cardiac injury. The Journal of pharmacology and experimental therapeutics 228, 761-768.

103. Foo, R.S., Chan, L.K., Kitsis, R.N., and Bennett, M.R. (2007). Ubiquitination and degradation of the anti-apoptotic protein ARC by MDM2. J Biol Chem 282, 5529-5535.

104. Foteinou, P.T., Greenstein, J.L., and Winslow, R.L. (2015). Mechanistic Investigation of the Arrhythmogenic Role of Oxidized CaMKII in the Heart. Biophysical journal 109, 838-849.

105. Fukuda, K., Davies, S.S., Nakajima, T., Ong, B.H., Kupershmidt, S., Fessel, J., Amarnath, V., Anderson, M.E., Boyden, P.A., Viswanathan, P.C., et al. (2005). Oxidative mediated lipid 
peroxidation recapitulates proarrhythmic effects on cardiac sodium channels. Circ Res 97, 12621269.

106. Gao, M.H., Lai, N.C., Roth, D.M., Zhou, J., Zhu, J., Anzai, T., Dalton, N., and Hammond, H.K. (1999). Adenylylcyclase increases responsiveness to catecholamine stimulation in transgenic mice. Circulation 99, 1618-1622.

107. Gill, J.S., McKenna, W.J., and Camm, A.J. (1995). Free radicals irreversibly decrease Ca2+ currents in isolated guinea-pig ventricular myocytes. European journal of pharmacology 292, 337-340.

108. Goldhaber, J.I. (1996). Free radicals enhance $\mathrm{Na}+\mathrm{Ca} 2+$ exchange in ventricular myocytes. The American journal of physiology 271, H823-833.

109. Goldhaber, J.I., Ji, S., Lamp, S.T., and Weiss, J.N. (1989). Effects of exogenous free radicals on electromechanical function and metabolism in isolated rabbit and guinea pig ventricle. Implications for ischemia and reperfusion injury. The Journal of clinical investigation 83, 18001809.

110. Goonasekera, S.A., Hammer, K., Auger-Messier, M., Bodi, I., Chen, X., Zhang, H., Reiken, S., Elrod, J.W., Correll, R.N., York, A.J., et al. (2012). Decreased cardiac L-type Ca(2)(+) channel activity induces hypertrophy and heart failure in mice. The Journal of clinical investigation 122 , 280-290.

111. Gopalakrishna, R., and Anderson, W.B. (1987). Susceptibility of protein kinase C to oxidative inactivation: loss of both phosphotransferase activity and phorbol diester binding. FEBS letters 225, 233-237.

112. Gopalakrishna, R., and Anderson, W.B. (1989). Ca2+- and phospholipid-independent activation of protein kinase $\mathrm{C}$ by selective oxidative modification of the regulatory domain. Proceedings of the National Academy of Sciences of the United States of America 86, 6758-6762.

113. Gorrini, C., Harris, I.S., and Mak, T.W. (2013). Modulation of oxidative stress as an anticancer strategy. Nature reviews Drug discovery 12, 931-947.

114. Greenberg, E.R., Baron, J.A., Karagas, M.R., Stukel, T.A., Nierenberg, D.W., Stevens, M.M., Mandel, J.S., and Haile, R.W. (1996). Mortality associated with low plasma concentration of beta carotene and the effect of oral supplementation. Jama 275, 699-703.

115. Griendling, K.K., and FitzGerald, G.A. (2003). Oxidative stress and cardiovascular injury: Part II: animal and human studies. Circulation 108, 2034-2040.

116. Grimm, M., and Brown, J.H. (2010). Beta-adrenergic receptor signaling in the heart: role of CaMKII. J Mol Cell Cardiol 48, 322-330.

117. Grueter, C.E., Abiria, S.A., Dzhura, I., Wu, Y., Ham, A.J., Mohler, P.J., Anderson, M.E., and Colbran, R.J. (2006). L-type Ca2+ channel facilitation mediated by phosphorylation of the beta subunit by CaMKII. Molecular cell 23, 641-650.

118. Gyorke, I., Hester, N., Jones, L.R., and Gyorke, S. (2004). The role of calsequestrin, triadin, and junctin in conferring cardiac ryanodine receptor responsiveness to luminal calcium. Biophysical journal 86, 2121-2128.

119. Ha, C.H., Kim, J.Y., Zhao, J., Wang, W., Jhun, B.S., Wong, C., and Jin, Z.G. (2010). PKA phosphorylates histone deacetylase 5 and prevents its nuclear export, leading to the inhibition of gene transcription and cardiomyocyte hypertrophy. Proceedings of the National Academy of Sciences of the United States of America 107, 15467-15472.

120. Hamawaki, M., Coffman, T.M., Lashus, A., Koide, M., Zile, M.R., Oliverio, M.I., DeFreyte, G., Cooper, G.t., and Carabello, B.A. (1998). Pressure-overload hypertrophy is unabated in mice devoid of AT1A receptors. The American journal of physiology 274, H868-873.

121. Hamilton, M.A., Stevenson, L.W., Luu, M., and Walden, J.A. (1990). Altered thyroid hormone metabolism in advanced heart failure. J Am Coll Cardiol 16, 91-95.

122. Hanatani, A., Yoshiyama, M., Kim, S., Omura, T., Toda, I., Akioka, K., Teragaki, M., Takeuchi, K., Iwao, H., and Takeda, T. (1995). Inhibition by angiotensin II type 1 receptor 
antagonist of cardiac phenotypic modulation after myocardial infarction. J Mol Cell Cardiol 27, 1905-1914.

123. Harada, K., Komuro, I., Shiojima, I., Hayashi, D., Kudoh, S., Mizuno, T., Kijima, K., Matsubara, H., Sugaya, T., Murakami, K., et al. (1998a). Pressure overload induces cardiac hypertrophy in angiotensin II type 1A receptor knockout mice. Circulation 97, 1952-1959.

124. Harada, K., Komuro, I., Zou, Y., Kudoh, S., Kijima, K., Matsubara, H., Sugaya, T., Murakami, K., and Yazaki, Y. (1998b). Acute pressure overload could induce hypertrophic responses in the heart of angiotensin II type 1a knockout mice. Circ Res 82, 779-785.

125. Hashambhoy, Y.L., Winslow, R.L., and Greenstein, J.L. (2011). CaMKII-dependent activation of late INa contributes to cellular arrhythmia in a model of the cardiac myocyte. Conference proceedings : Annual International Conference of the IEEE Engineering in Medicine and Biology Society IEEE Engineering in Medicine and Biology Society Annual Conference 2011, 4665-4668.

126. Haywood, G.A., Tsao, P.S., von der Leyen, H.E., Mann, M.J., Keeling, P.J., Trindade, P.T., Lewis, N.P., Byrne, C.D., Rickenbacher, P.R., Bishopric, N.H., et al. (1996). Expression of inducible nitric oxide synthase in human heart failure. Circulation 93, 1087-1094.

127. He, J., Conklin, M.W., Foell, J.D., Wolff, M.R., Haworth, R.A., Coronado, R., and Kamp, T.J. (2001). Reduction in density of transverse tubules and L-type $\mathrm{Ca}(2+)$ channels in canine tachycardia-induced heart failure. Cardiovasc Res 49, 298-307.

128. Heart Protection Study Collaborative, G. (2002). MRC/BHF Heart Protection Study of antioxidant vitamin supplementation in 20,536 high-risk individuals: a randomised placebocontrolled trial. Lancet 360, 23-33.

129. Hennekens, C.H., Buring, J.E., Manson, J.E., Stampfer, M., Rosner, B., Cook, N.R., Belanger, C., LaMotte, F., Gaziano, J.M., Ridker, P.M., et al. (1996). Lack of effect of long-term supplementation with beta carotene on the incidence of malignant neoplasms and cardiovascular disease. The New England journal of medicine 334, 1145-1149.

130. Heymes, C., Bendall, J.K., Ratajczak, P., Cave, A.C., Samuel, J.L., Hasenfuss, G., and Shah, A.M. (2003). Increased myocardial NADPH oxidase activity in human heart failure. J Am Coll Cardiol 41, 2164-2171.

131. Hingtgen, S.D., Tian, X., Yang, J., Dunlay, S.M., Peek, A.S., Wu, Y., Sharma, R.V., Engelhardt, J.F., and Davisson, R.L. (2006). Nox2-containing NADPH oxidase and Akt activation play a key role in angiotensin II-induced cardiomyocyte hypertrophy. Physiological genomics 26, 180-191.

132. Hirano, Y., Moscucci, A., and January, C.T. (1992). Direct measurement of L-type Ca2+ window current in heart cells. Circ Res 70, 445-455.

133. Hiraoka, Y., Kishimoto, C., Takada, H., Nakamura, M., Kurokawa, M., Ochiai, H., and Shiraki, K. (1996). Nitric oxide and murine coxsackievirus B3 myocarditis: aggravation of myocarditis by inhibition of nitric oxide synthase. J Am Coll Cardiol 28, 1610-1615.

134. Hirotani, S., Otsu, K., Nishida, K., Higuchi, Y., Morita, T., Nakayama, H., Yamaguchi, O., Mano, T., Matsumura, Y., Ueno, H., et al. (2002). Involvement of nuclear factor-kappaB and apoptosis signal-regulating kinase 1 in G-protein-coupled receptor agonist-induced cardiomyocyte hypertrophy. Circulation 105, 509-515.

135. Hodis, H.N., Mack, W.J., LaBree, L., Mahrer, P.R., Sevanian, A., Liu, C.R., Liu, C.H., Hwang, J., Selzer, R.H., Azen, S.P., et al. (2002). Alpha-tocopherol supplementation in healthy individuals reduces low-density lipoprotein oxidation but not atherosclerosis: the Vitamin E Atherosclerosis Prevention Study (VEAPS). Circulation 106, 1453-1459.

136. Holmgren, A. (1995). Thioredoxin structure and mechanism: conformational changes on oxidation of the active-site sulfhydryls to a disulfide. Structure 3, 239-243.

137. Hu, H., Chiamvimonvat, N., Yamagishi, T., and Marban, E. (1997). Direct inhibition of expressed cardiac L-type Ca2+ channels by S-nitrosothiol nitric oxide donors. Circ Res 81, 742752. 
138. Hudmon, A., Schulman, H., Kim, J., Maltez, J.M., Tsien, R.W., and Pitt, G.S. (2005). CaMKII tethers to L-type $\mathrm{Ca} 2+$ channels, establishing a local and dedicated integrator of $\mathrm{Ca} 2+$ signals for facilitation. The Journal of cell biology 171, 537-547.

139. Ichiyanagi, O., Ishii, K., and Endoh, M. (2002). Angiotensin II increases L-type Ca2+ current in gramicidin D-perforated adult rabbit ventricular myocytes: comparison with conventional patch-clamp method. Pflugers Archiv : European journal of physiology 444, 107116.

140. Ide, T., Tsutsui, H., Kinugawa, S., Suematsu, N., Hayashidani, S., Ichikawa, K., Utsumi, H., Machida, Y., Egashira, K., and Takeshita, A. (2000). Direct evidence for increased hydroxyl radicals originating from superoxide in the failing myocardium. Circ Res 86, 152-157.

141. Ide, T., Tsutsui, H., Kinugawa, S., Utsumi, H., Kang, D., Hattori, N., Uchida, K., Arimura, K., Egashira, K., and Takeshita, A. (1999). Mitochondrial electron transport complex I is a potential source of oxygen free radicals in the failing myocardium. Circ Res 85, 357-363.

142. Iwase, M., Bishop, S.P., Uechi, M., Vatner, D.E., Shannon, R.P., Kudej, R.K., Wight, D.C., Wagner, T.E., Ishikawa, Y., Homcy, C.J., et al. (1996). Adverse effects of chronic endogenous sympathetic drive induced by cardiac GS alpha overexpression. Circ Res 78, 517-524.

143. January, C.T., and Riddle, J.M. (1989). Early afterdepolarizations: mechanism of induction and block. A role for L-type Ca2+ current. Circ Res 64, 977-990.

144. Jastroch, M., Divakaruni, A.S., Mookerjee, S., Treberg, J.R., and Brand, M.D. (2010). Mitochondrial proton and electron leaks. Essays in biochemistry 47, 53-67.

145. Johnston, C.I. (1992). Franz Volhard Lecture. Renin-angiotensin system: a dual tissue and hormonal system for cardiovascular control. Journal of hypertension Supplement : official journal of the International Society of Hypertension 10, S13-26.

146. Jones, B.W., Brunet, S., Gilbert, M.L., Nichols, C.B., Su, T., Westenbroek, R.E., Scott, J.D., Catterall, W.A., and McKnight, G.S. (2012). Cardiomyocytes from AKAP7 knockout mice respond normally to adrenergic stimulation. Proceedings of the National Academy of Sciences of the United States of America 109, 17099-17104.

147. Jorgensen, A.O., Shen, A.C., Arnold, W., McPherson, P.S., and Campbell, K.P. (1993). The Ca2+-release channel/ryanodine receptor is localized in junctional and corbular sarcoplasmic reticulum in cardiac muscle. The Journal of cell biology 120, 969-980.

148. Josephson, I.R., Sanchez-Chapula, J., and Brown, A.M. (1984). A comparison of calcium currents in rat and guinea pig single ventricular cells. Circ Res 54, 144-156.

149. Kahan, T., and Bergfeldt, L. (2005). Left ventricular hypertrophy in hypertension: its arrhythmogenic potential. Heart 91, 250-256.

150. Kaludercic, N., Takimoto, E., Nagayama, T., Feng, N., Lai, E.W., Bedja, D., Chen, K., Gabrielson, K.L., Blakely, R.D., Shih, J.C., et al. (2010). Monoamine oxidase A-mediated enhanced catabolism of norepinephrine contributes to adverse remodeling and pump failure in hearts with pressure overload. Circ Res 106, 193-202.

151. Kato, M., and Kako, K.J. (1988). Na+/Ca2+ exchange of isolated sarcolemmal membrane: effects of insulin, oxidants and insulin deficiency. Molecular and cellular biochemistry 83, 15-25.

152. Kawakami, M., and Okabe, E. (1998). Superoxide anion radical-triggered Ca2+ release from cardiac sarcoplasmic reticulum through ryanodine receptor $\mathrm{Ca} 2+$ channel. Mol Pharmacol 53, 497-503.

153. Khafizov, K., Lattanzi, G., and Carloni, P. (2009). G protein inactive and active forms investigated by simulation methods. Proteins 75, 919-930.

154. Kim, C., Xuong, N.H., and Taylor, S.S. (2005). Crystal structure of a complex between the catalytic and regulatory (RIalpha) subunits of PKA. Science 307, 690-696.

155. Kim, J.S., Diebold, B.A., Babior, B.M., Knaus, U.G., and Bokoch, G.M. (2007). Regulation of Nox1 activity via protein kinase A-mediated phosphorylation of NoxA1 and 14-3-3 binding. J Biol Chem 282, 34787-34800. 
156. Kim, M.S., and Akera, T. (1987). 02 free radicals: cause of ischemia-reperfusion injury to cardiac Na+-K+-ATPase. The American journal of physiology 252, H252-257.

157. Kim, S., Ohta, K., Hamaguchi, A., Yukimura, T., Miura, K., and Iwao, H. (1995). Angiotensin II induces cardiac phenotypic modulation and remodeling in vivo in rats. Hypertension 25, 12521259.

158. Kim, S.Y., Kim, S.J., Kim, B.J., Rah, S.Y., Chung, S.M., Im, M.J., and Kim, U.H. (2006). Doxorubicin-induced reactive oxygen species generation and intracellular $\mathrm{Ca} 2+$ increase are reciprocally modulated in rat cardiomyocytes. Experimental \& molecular medicine 38, 535-545.

159. Kloner, R.A., Hale, S.L., Dai, W., Gorman, R.C., Shuto, T., Koomalsingh, K.J., Gorman, J.H., 3rd, Sloan, R.C., Frasier, C.R., Watson, C.A., et al. (2012). Reduction of ischemia/reperfusion injury with bendavia, a mitochondria-targeting cytoprotective Peptide. Journal of the American Heart Association 1, e001644.

160. Koncz, I., Gurabi, Z., Patocskai, B., Panama, B.K., Szel, T., Hu, D., Barajas-Martinez, H., and Antzelevitch, C. (2014). Mechanisms underlying the development of the electrocardiographic and arrhythmic manifestations of early repolarization syndrome. J Mol Cell Cardiol 68, 20-28.

161. Kornfeld, O.S., Hwang, S., Disatnik, M.H., Chen, C.H., Qvit, N., and Mochly-Rosen, D. (2015). Mitochondrial reactive oxygen species at the heart of the matter: new therapeutic approaches for cardiovascular diseases. Circ Res 116, 1783-1799.

162. Koval, O.M., Guan, X., Wu, Y., Joiner, M.L., Gao, Z., Chen, B., Grumbach, I.M., Luczak, E.D., Colbran, R.J., Song, L.S., et al. (2010). CaV1.2 beta-subunit coordinates CaMKII-triggered cardiomyocyte death and afterdepolarizations. Proceedings of the National Academy of Sciences of the United States of America 107, 4996-5000.

163. Koval, O.M., Snyder, J.S., Wolf, R.M., Pavlovicz, R.E., Glynn, P., Curran, J., Leymaster, N.D., Dun, W., Wright, P.J., Cardona, N., et al. (2012). Ca2+/calmodulin-dependent protein kinase IIbased regulation of voltage-gated $\mathrm{Na}+$ channel in cardiac disease. Circulation 126, 2084-2094.

164. Krall, J., Tasken, K., Staheli, J., Jahnsen, T., and Movsesian, M.A. (1999). Identification and quantitation of cAMP-dependent protein kinase $\mathrm{R}$ subunit isoforms in subcellular fractions of failing human myocardium. J Mol Cell Cardiol 31, 971-980.

165. Kukreja, R.C., Okabe, E., Schrier, G.M., and Hess, M.L. (1988). Oxygen radical-mediated lipid peroxidation and inhibition of Ca2+-ATPase activity of cardiac sarcoplasmic reticulum. Archives of biochemistry and biophysics 261, 447-457.

166. Kukreja, R.C., Weaver, A.B., and Hess, M.L. (1990). Sarcolemmal Na(+)-K(+)-ATPase: inactivation by neutrophil-derived free radicals and oxidants. The American journal of physiology 259, H1330-1336.

167. Lacampagne, A., Duittoz, A., Bolanos, P., Peineau, N., and Argibay, J.A. (1995). Effect of sulfhydryl oxidation on ionic and gating currents associated with L-type calcium channels in isolated guinea-pig ventricular myocytes. Cardiovasc Res 30, 799-806.

168. Lassegue, B., San Martin, A., and Griendling, K.K. (2012). Biochemistry, physiology, and pathophysiology of NADPH oxidases in the cardiovascular system. Circ Res 110, 1364-1390.

169. Laurita, K.R., and Katra, R.P. (2005). Delayed after depolarization-mediated triggered activity associated with slow calcium sequestration near the endocardium. Journal of cardiovascular electrophysiology 16, 418-424.

170. Laursen, J.B., Rajagopalan, S., Galis, Z., Tarpey, M., Freeman, B.A., and Harrison, D.G. (1997). Role of superoxide in angiotensin II-induced but not catecholamine-induced hypertension. Circulation 95, 588-593.

171. Le Peuch, C.J., Haiech, J., and Demaille, J.G. (1979). Concerted regulation of cardiac sarcoplasmic reticulum calcium transport by cyclic adenosine monophosphate dependent and calcium--calmodulin-dependent phosphorylations. Biochemistry 18, 5150-5157.

172. Lefer, A.M., Tsao, P., Aoki, N., and Palladino, M.A., Jr. (1990). Mediation of cardioprotection by transforming growth factor-beta. Science 249, 61-64. 
173. Lefkowitz, R.J. (1998). G protein-coupled receptors. III. New roles for receptor kinases and beta-arrestins in receptor signaling and desensitization. J Biol Chem 273, 18677-18680.

174. Leroy, J., Richter, W., Mika, D., Castro, L.R., Abi-Gerges, A., Xie, M., Scheitrum, C., Lefebvre, F., Schittl, J., Mateo, P., et al. (2011). Phosphodiesterase 4B in the cardiac L-type Ca(2)(+) channel complex regulates $\mathrm{Ca}(2)(+)$ current and protects against ventricular arrhythmias in mice. The Journal of clinical investigation 121, 2651-2661.

175. Li, N., and Wehrens, X.H. (2010). Programmed electrical stimulation in mice. Journal of visualized experiments : JoVE.

176. Li, Y., Huang, T.T., Carlson, E.J., Melov, S., Ursell, P.C., Olson, J.L., Noble, L.J., Yoshimura, M.P., Berger, C., Chan, P.H., et al. (1995). Dilated cardiomyopathy and neonatal lethality in mutant mice lacking manganese superoxide dismutase. Nature genetics 11, 376-381.

177. Liggett, S.B., Tepe, N.M., Lorenz, J.N., Canning, A.M., Jantz, T.D., Mitarai, S., Yatani, A., and Dorn, G.W., 2nd (2000). Early and delayed consequences of beta(2)-adrenergic receptor overexpression in mouse hearts: critical role for expression level. Circulation 101, 1707-1714.

178. Liu, G., Abramson, J.J., Zable, A.C., and Pessah, I.N. (1994). Direct evidence for the existence and functional role of hyperreactive sulfhydryls on the ryanodine receptor-triadin complex selectively labeled by the coumarin maleimide 7-diethylamino-3-(4'maleimidylphenyl)-4-methylcoumarin. Mol Pharmacol 45, 189-200.

179. Liu, Y., Jurman, M.E., and Yellen, G. (1996). Dynamic rearrangement of the outer mouth of a K+ channel during gating. Neuron $16,859-867$.

180. Lohse, M.J., and Engelhardt, S. (2001). Protein kinase a transgenes: the many faces of cAMP. Circ Res 89, 938-940.

181. Lombardi, D., Gordon, K.L., Polinsky, P., Suga, S., Schwartz, S.M., and Johnson, R.J. (1999). Salt-sensitive hypertension develops after short-term exposure to Angiotensin II. Hypertension 33, 1013-1019.

182. Lonn, E., Yusuf, S., Hoogwerf, B., Pogue, J., Yi, Q., Zinman, B., Bosch, J., Dagenais, G., Mann, J.F., Gerstein, H.C., et al. (2002). Effects of vitamin E on cardiovascular and microvascular outcomes in high-risk patients with diabetes: results of the HOPE study and MICRO-HOPE substudy. Diabetes care 25, 1919-1927.

183. Looi, Y.H., Grieve, D.J., Siva, A., Walker, S.J., Anilkumar, N., Cave, A.C., Marber, M., Monaghan, M.J., and Shah, A.M. (2008). Involvement of Nox2 NADPH oxidase in adverse cardiac remodeling after myocardial infarction. Hypertension 51, 319-325.

184. Lowenstein, C.J., Hill, S.L., Lafond-Walker, A., Wu, J., Allen, G., Landavere, M., Rose, N.R., and Herskowitz, A. (1996). Nitric oxide inhibits viral replication in murine myocarditis. The Journal of clinical investigation 97, 1837-1843.

185. Lutz, S., Mura, R., Baltus, D., Movsesian, M., Kubler, W., and Niroomand, F. (2001). Increased activity of membrane-associated nucleoside diphosphate kinase and inhibition of cAMP synthesis in failing human myocardium. Cardiovasc Res 49, 48-55.

186. Maack, C., and Bohm, M. (2011). Targeting mitochondrial oxidative stress in heart failure throttling the afterburner. J Am Coll Cardiol 58, 83-86.

187. Madamanchi, N.R., Vendrov, A., and Runge, M.S. (2005). Oxidative stress and vascular disease. Arteriosclerosis, thrombosis, and vascular biology 25, 29-38.

188. Maier, L.S., and Bers, D.M. (2002). Calcium, calmodulin, and calcium-calmodulin kinase II: heartbeat to heartbeat and beyond. J Mol Cell Cardiol 34, 919-939.

189. Maier, L.S., and Bers, D.M. (2007). Role of Ca2+/calmodulin-dependent protein kinase (CaMK) in excitation-contraction coupling in the heart. Cardiovasc Res 73, 631-640.

190. Maier, L.S., Zhang, T., Chen, L., DeSantiago, J., Brown, J.H., and Bers, D.M. (2003). Transgenic CaMKIIdeltaC overexpression uniquely alters cardiac myocyte $\mathrm{Ca} 2+$ handling: reduced SR Ca2+ load and activated SR Ca2+ release. Circ Res 92, 904-911. 
191. Mari, M., Morales, A., Colell, A., Garcia-Ruiz, C., and Fernandez-Checa, J.C. (2009). Mitochondrial glutathione, a key survival antioxidant. Antioxidants \& redox signaling 11, 26852700.

192. Marx, S.O., Reiken, S., Hisamatsu, Y., Jayaraman, T., Burkhoff, D., Rosemblit, N., and Marks, A.R. (2000). PKA phosphorylation dissociates FKBP12.6 from the calcium release channel (ryanodine receptor): defective regulation in failing hearts. Cell 101, 365-376.

193. Mates, J.M., Perez-Gomez, C., and Nunez de Castro, I. (1999). Antioxidant enzymes and human diseases. Clinical biochemistry 32, 595-603.

194. Maxwell, S.R. (1995). Prospects for the use of antioxidant therapies. Drugs 49, 345-361.

195. Mazzolai, L., Pedrazzini, T., Nicoud, F., Gabbiani, G., Brunner, H.R., and Nussberger, J. (2000). Increased cardiac angiotensin II levels induce right and left ventricular hypertrophy in normotensive mice. Hypertension 35, 985-991.

196. Mellin, V., Isabelle, M., Oudot, A., Vergely-Vandriesse, C., Monteil, C., Di Meglio, B., Henry, J.P., Dautreaux, B., Rochette, L., Thuillez, C., et al. (2005). Transient reduction in myocardial free oxygen radical levels is involved in the improved cardiac function and structure after long-term allopurinol treatment initiated in established chronic heart failure. European heart journal 26, 1544-1550.

197. Melov, S. (2002). Animal models of oxidative stress, aging, and therapeutic antioxidant interventions. The international journal of biochemistry \& cell biology 34, 1395-1400.

198. Mery, P.F., Pavoine, C., Belhassen, L., Pecker, F., and Fischmeister, R. (1993). Nitric oxide regulates cardiac $\mathrm{Ca} 2+$ current. Involvement of cGMP-inhibited and cGMP-stimulated phosphodiesterases through guanylyl cyclase activation. J Biol Chem 268, 26286-26295.

199. Milano, C.A., Allen, L.F., Rockman, H.A., Dolber, P.C., McMinn, T.R., Chien, K.R., Johnson, T.D., Bond, R.A., and Lefkowitz, R.J. (1994). Enhanced myocardial function in transgenic mice overexpressing the beta 2-adrenergic receptor. Science 264, 582-586.

200. Minamisawa, S., Hoshijima, M., Chu, G., Ward, C.A., Frank, K., Gu, Y., Martone, M.E., Wang, Y., Ross, J., Jr., Kranias, E.G., et al. (1999). Chronic phospholamban-sarcoplasmic reticulum calcium ATPase interaction is the critical calcium cycling defect in dilated cardiomyopathy. Cell 99, 313-322.

201. Miyamoto, M.I., del Monte, F., Schmidt, U., DiSalvo, T.S., Kang, Z.B., Matsui, T., Guerrero, J.L., Gwathmey, J.K., Rosenzweig, A., and Hajjar, R.J. (2000). Adenoviral gene transfer of SERCA2a improves left-ventricular function in aortic-banded rats in transition to heart failure. Proceedings of the National Academy of Sciences of the United States of America 97, 793-798.

202. Miyata, S., and Haneda, T. (1994). Hypertrophic growth of cultured neonatal rat heart cells mediated by type 1 angiotensin II receptor. The American journal of physiology 266, H24432451.

203. Mochizuki, M., Yano, M., Oda, T., Tateishi, H., Kobayashi, S., Yamamoto, T., Ikeda, Y., Ohkusa, T., Ikemoto, N., and Matsuzaki, M. (2007). Scavenging free radicals by low-dose carvedilol prevents redox-dependent $\mathrm{Ca} 2+$ leak via stabilization of ryanodine receptor in heart failure. J Am Coll Cardiol 49, 1722-1732.

204. Molkentin, J.D. (2000). Calcineurin and beyond: cardiac hypertrophic signaling. Circ Res 87, 731-738.

205. Molkentin, J.D., Lu, J.R., Antos, C.L., Markham, B., Richardson, J., Robbins, J., Grant, S.R., and Olson, E.N. (1998). A calcineurin-dependent transcriptional pathway for cardiac hypertrophy. Cell 93, 215-228.

206. Morris, T.E., and Sulakhe, P.V. (1997). Sarcoplasmic reticulum Ca(2+)-pump dysfunction in rat cardiomyocytes briefly exposed to hydroxyl radicals. Free radical biology \& medicine 22, 37-47.

207. Mukherjee, R., and Spinale, F.G. (1998). L-type calcium channel abundance and function with cardiac hypertrophy and failure: a review. J Mol Cell Cardiol 30, 1899-1916. 
208. Mulrooney, D.A., Yeazel, M.W., Kawashima, T., Mertens, A.C., Mitby, P., Stovall, M., Donaldson, S.S., Green, D.M., Sklar, C.A., Robison, L.L., et al. (2009). Cardiac outcomes in a cohort of adult survivors of childhood and adolescent cancer: retrospective analysis of the Childhood Cancer Survivor Study cohort. Bmj 339, b4606.

209. Murphy, B.J., Rogers, J., Perdichizzi, A.P., Colvin, A.A., and Catterall, W.A. (1996). cAMPdependent phosphorylation of two sites in the alpha subunit of the cardiac sodium channel. J Biol Chem 271, 28837-28843.

210. Nagy, N., Kormos, A., Kohajda, Z., Szebeni, A., Szepesi, J., Pollesello, P., Levijoki, J., Acsai, K., Virag, L., Nanasi, P.P., et al. (2014). Selective $\mathrm{Na}(+) / \mathrm{Ca}(2+)$ exchanger inhibition prevents $\mathrm{Ca}(2+)$ overload-induced triggered arrhythmias. British journal of pharmacology 171, 5665-5681.

211. Newlon, M.G., Roy, M., Morikis, D., Carr, D.W., Westphal, R., Scott, J.D., and Jennings, P.A. (2001). A novel mechanism of PKA anchoring revealed by solution structures of anchoring complexes. The EMBO journal 20, 1651-1662.

212. Nicholls, M.G., Robertson, J.I., and Inagami, T. (2001). The renin-angiotensin system in the twenty-first century. Blood pressure 10, 327-343.

213. Nikolaev, V.O., Moshkov, A., Lyon, A.R., Miragoli, M., Novak, P., Paur, H., Lohse, M.J., Korchev, Y.E., Harding, S.E., and Gorelik, J. (2010). Beta2-adrenergic receptor redistribution in heart failure changes cAMP compartmentation. Science 327, 1653-1657.

214. Nishida, M., Tanabe, S., Maruyama, Y., Mangmool, S., Urayama, K., Nagamatsu, Y., Takagahara, S., Turner, J.H., Kozasa, T., Kobayashi, H., et al. (2005). G alpha 12/13- and reactive oxygen species-dependent activation of c-Jun NH2-terminal kinase and p38 mitogen-activated protein kinase by angiotensin receptor stimulation in rat neonatal cardiomyocytes. J Biol Chem $280,18434-18441$.

215. Norton, G.R., Woodiwiss, A.J., Gaasch, W.H., Mela, T., Chung, E.S., Aurigemma, G.P., and Meyer, T.E. (2002). Heart failure in pressure overload hypertrophy. The relative roles of ventricular remodeling and myocardial dysfunction. J Am Coll Cardiol 39, 664-671.

216. Nowycky, M.C., Fox, A.P., and Tsien, R.W. (1985). Three types of neuronal calcium channel with different calcium agonist sensitivity. Nature 316, 440-443.

217. Ogielska, E.M., Zagotta, W.N., Hoshi, T., Heinemann, S.H., Haab, J., and Aldrich, R.W. (1995). Cooperative subunit interactions in C-type inactivation of K channels. Biophysical journal 69, 2449-2457.

218. Ojamaa, K., Kenessey, A., Shenoy, R., and Klein, I. (2000). Thyroid hormone metabolism and cardiac gene expression after acute myocardial infarction in the rat. American journal of physiology Endocrinology and metabolism 279, E1319-1324.

219. Olson, E.N., and Molkentin, J.D. (1999). Prevention of cardiac hypertrophy by calcineurin inhibition: hope or hype? Circ Res 84, 623-632.

220. Omenn, G.S., Goodman, G.E., Thornquist, M.D., Balmes, J., Cullen, M.R., Glass, A., Keogh, J.P., Meyskens, F.L., Valanis, B., Williams, J.H., et al. (1996). Effects of a combination of beta carotene and vitamin A on lung cancer and cardiovascular disease. The New England journal of medicine 334, 1150-1155.

221. Orellana, S.A., and McKnight, G.S. (1990). The S49 Kin- cell line transcribes and translates a functional mRNA coding for the catalytic subunit of cAMP-dependent protein kinase. J Biol Chem 265, 3048-3053.

222. Orrenius, S., McConkey, D.J., Bellomo, G., and Nicotera, P. (1989). Role of Ca2+ in toxic cell killing. Trends in pharmacological sciences 10, 281-285.

223. Orrenius, S., and Nicotera, P. (1994). The calcium ion and cell death. Journal of neural transmission Supplementum 43, 1-11.

224. Owusu-Ansah, E., and Banerjee, U. (2009). Reactive oxygen species prime Drosophila haematopoietic progenitors for differentiation. Nature 461, 537-541. 
225. Pacher, P., Beckman, J.S., and Liaudet, L. (2007). Nitric oxide and peroxynitrite in health and disease. Physiol Rev 87, 315-424.

226. Padayatty, S.J., Katz, A., Wang, Y., Eck, P., Kwon, O., Lee, J.H., Chen, S., Corpe, C., Dutta, A., Dutta, S.K., et al. (2003). Vitamin C as an antioxidant: evaluation of its role in disease prevention. Journal of the American College of Nutrition 22, 18-35.

227. Palomeque, J., Rueda, O.V., Sapia, L., Valverde, C.A., Salas, M., Petroff, M.V., and Mattiazzi, A. (2009). Angiotensin II-induced oxidative stress resets the $\mathrm{Ca} 2+$ dependence of $\mathrm{Ca} 2+-$ calmodulin protein kinase II and promotes a death pathway conserved across different species. Circ Res 105, 1204-1212.

228. Patel, C., Salahuddin, M., Jones, A., Patel, A., Yan, G.X., and Kowey, P.R. (2011). Atrial fibrillation: pharmacological therapy. Current problems in cardiology 36, 87-120.

229. Pelzmann, B., Schaffer, P., Bernhart, E., Lang, P., Machler, H., Rigler, B., and Koidl, B. (1998). L-type calcium current in human ventricular myocytes at a physiological temperature from children with tetralogy of Fallot. Cardiovasc Res 38, 424-432.

230. Pereira, L., Cheng, H., Lao, D.H., Na, L., van Oort, R.J., Brown, J.H., Wehrens, X.H., Chen, J., and Bers, D.M. (2013). Epac2 mediates cardiac beta1-adrenergic-dependent sarcoplasmic reticulum Ca2+ leak and arrhythmia. Circulation 127, 913-922.

231. Perez, N.G., Hashimoto, K., McCune, S., Altschuld, R.A., and Marban, E. (1999). Origin of contractile dysfunction in heart failure: calcium cycling versus myofilaments. Circulation 99, $1077-1083$

232. Petroff, M.G., Kim, S.H., Pepe, S., Dessy, C., Marban, E., Balligand, J.L., and Sollott, S.J. (2001). Endogenous nitric oxide mechanisms mediate the stretch dependence of Ca2+ release in cardiomyocytes. Nature cell biology 3, 867-873.

233. Pham-Huy, L.A., He, H., and Pham-Huy, C. (2008). Free radicals, antioxidants in disease and health. International journal of biomedical science : IJBS 4, 89-96.

234. Phillips, M.I., Speakman, E.A., and Kimura, B. (1993). Levels of angiotensin and molecular biology of the tissue renin angiotensin systems. Regulatory peptides 43, 1-20.

235. Picht, E., Zima, A.V., Blatter, L.A., and Bers, D.M. (2007). SparkMaster: automated calcium spark analysis with ImageJ. American journal of physiology Cell physiology 293, C1073-1081.

236. Pieske, B., Maier, L.S., Piacentino, V., 3rd, Weisser, J., Hasenfuss, G., and Houser, S. (2002). Rate dependence of $[\mathrm{Na}+] \mathrm{i}$ and contractility in nonfailing and failing human myocardium. Circulation 106, 447-453.

237. Pogwizd, S.M., Hoyt, R.H., Saffitz, J.E., Corr, P.B., Cox, J.L., and Cain, M.E. (1992). Reentrant and focal mechanisms underlying ventricular tachycardia in the human heart. Circulation 86, 1872-1887.

238. Pogwizd, S.M., McKenzie, J.P., and Cain, M.E. (1998). Mechanisms underlying spontaneous and induced ventricular arrhythmias in patients with idiopathic dilated cardiomyopathy. Circulation 98, 2404-2414.

239. Poole, L.B., and Nelson, K.J. (2008). Discovering mechanisms of signaling-mediated cysteine oxidation. Current opinion in chemical biology 12, 18-24.

240. Poteet-Smith, C.E., Shabb, J.B., Francis, S.H., and Corbin, J.D. (1997). Identification of critical determinants for autoinhibition in the pseudosubstrate region of type I alpha cAMPdependent protein kinase. J Biol Chem 272, 379-388.

241. Prosser, B.L., Ward, C.W., and Lederer, W.J. (2011). X-ROS signaling: rapid mechanochemo transduction in heart. Science 333, 1440-1445.

242. Przyklenk, K., and Kloner, R.A. (1989). "Reperfusion injury" by oxygen-derived free radicals? Effect of superoxide dismutase plus catalase, given at the time of reperfusion, on myocardial infarct size, contractile function, coronary microvasculature, and regional myocardial blood flow. Circ Res 64, 86-96. 
243. Puett, D.W., Forman, M.B., Cates, C.U., Wilson, B.H., Hande, K.R., Friesinger, G.C., and Virmani, R. (1987). Oxypurinol limits myocardial stunning but does not reduce infarct size after reperfusion. Circulation 76, 678-686.

244. Purohit, A., Rokita, A.G., Guan, X., Chen, B., Koval, O.M., Voigt, N., Neef, S., Sowa, T., Gao, Z., Luczak, E.D., et al. (2013). Oxidized $\mathrm{Ca}(2+) /$ calmodulin-dependent protein kinase II triggers atrial fibrillation. Circulation 128, 1748-1757.

245. Rajagopalan, S., Kurz, S., Munzel, T., Tarpey, M., Freeman, B.A., Griendling, K.K., and Harrison, D.G. (1996). Angiotensin II-mediated hypertension in the rat increases vascular superoxide production via membrane NADH/NADPH oxidase activation. Contribution to alterations of vasomotor tone. The Journal of clinical investigation 97, 1916-1923.

246. Ramirez, M.T., Zhao, X.L., Schulman, H., and Brown, J.H. (1997). The nuclear deltaB isoform of $\mathrm{Ca} 2+/$ calmodulin-dependent protein kinase II regulates atrial natriuretic factor gene expression in ventricular myocytes. J Biol Chem 272, 31203-31208.

247. Ramirez-Correa, G.A., Cortassa, S., Stanley, B., Gao, W.D., and Murphy, A.M. (2010). Calcium sensitivity, force frequency relationship and cardiac troponin I: critical role of PKA and PKC phosphorylation sites. J Mol Cell Cardiol 48, 943-953.

248. Rapola, J.M., Virtamo, J., Ripatti, S., Huttunen, J.K., Albanes, D., Taylor, P.R., and Heinonen, O.P. (1997). Randomised trial of alpha-tocopherol and beta-carotene supplements on incidence of major coronary events in men with previous myocardial infarction. Lancet 349, 1715-1720.

249. Reeves, J.P., Bailey, C.A., and Hale, C.C. (1986). Redox modification of sodium-calcium exchange activity in cardiac sarcolemmal vesicles. J Biol Chem 261, 4948-4955.

250. Reichel, H., and Bleichert, A. (1959). Excitation-contraction coupling in heart muscle. Nature 183, 826-827.

251. Remondino, A., Kwon, S.H., Communal, C., Pimentel, D.R., Sawyer, D.B., Singh, K., and Colucci, W.S. (2003). Beta-adrenergic receptor-stimulated apoptosis in cardiac myocytes is mediated by reactive oxygen species/c-Jun NH2-terminal kinase-dependent activation of the mitochondrial pathway. Circ Res 92, 136-138.

252. Reuter, H., and Scholz, H. (1977). A study of the ion selectivity and the kinetic properties of the calcium dependent slow inward current in mammalian cardiac muscle. The Journal of physiology 264, 17-47.

253. Robinson, K.M., Janes, M.S., and Beckman, J.S. (2008). The selective detection of mitochondrial superoxide by live cell imaging. Nature protocols 3, 941-947.

254. Rockman, H.A., Ross, R.S., Harris, A.N., Knowlton, K.U., Steinhelper, M.E., Field, L.J., Ross, J., Jr., and Chien, K.R. (1991). Segregation of atrial-specific and inducible expression of an atrial natriuretic factor transgene in an in vivo murine model of cardiac hypertrophy. Proceedings of the National Academy of Sciences of the United States of America 88, 8277-8281.

255. Rohrer, D.K., Desai, K.H., Jasper, J.R., Stevens, M.E., Regula, D.P., Jr., Barsh, G.S., Bernstein, D., and Kobilka, B.K. (1996). Targeted disruption of the mouse beta1-adrenergic receptor gene: developmental and cardiovascular effects. Proceedings of the National Academy of Sciences of the United States of America 93, 7375-7380.

256. Roth, D.M., Gao, M.H., Lai, N.C., Drumm, J., Dalton, N., Zhou, J.Y., Zhu, J., Entrikin, D., and Hammond, H.K. (1999). Cardiac-directed adenylyl cyclase expression improves heart function in murine cardiomyopathy. Circulation 99, 3099-3102.

257. Rowe, G.T., Eaton, L.R., and Hess, M.L. (1984). Neutrophil-derived, oxygen free radicalmediated cardiovascular dysfunction. J Mol Cell Cardiol 16, 1075-1079.

258. Ruiz-Ortega, M., Lorenzo, O., Ruperez, M., Esteban, V., Suzuki, Y., Mezzano, S., Plaza, J.J., and Egido, J. (2001). Role of the renin-angiotensin system in vascular diseases: expanding the field. Hypertension 38, 1382-1387.

259. Saavedra, W.F., Paolocci, N., St John, M.E., Skaf, M.W., Stewart, G.C., Xie, J.S., Harrison, R.W., Zeichner, J., Mudrick, D., Marban, E., et al. (2002). Imbalance between xanthine oxidase and 
nitric oxide synthase signaling pathways underlies mechanoenergetic uncoupling in the failing heart. Circ Res 90, 297-304.

260. Sachse, A., and Wolf, G. (2007). Angiotensin II-induced reactive oxygen species and the kidney. Journal of the American Society of Nephrology : JASN 18, 2439-2446.

261. Sadoshima, J., and Izumo, S. (1993a). Molecular characterization of angiotensin II-induced hypertrophy of cardiac myocytes and hyperplasia of cardiac fibroblasts. Critical role of the AT1 receptor subtype. Circ Res 73, 413-423.

262. Sadoshima, J., and Izumo, S. (1993b). Signal transduction pathways of angiotensin II-induced c-fos gene expression in cardiac myocytes in vitro. Roles of phospholipid-derived second messengers. Circ Res 73, 424-438.

263. Sag, C.M., Kohler, A.C., Anderson, M.E., Backs, J., and Maier, L.S. (2011). CaMKIIdependent SR Ca leak contributes to doxorubicin-induced impaired Ca handling in isolated cardiac myocytes. J Mol Cell Cardiol 51, 749-759.

264. Sag, C.M., Wadsack, D.P., Khabbazzadeh, S., Abesser, M., Grefe, C., Neumann, K., Opiela, M.K., Backs, J., Olson, E.N., Brown, J.H., et al. (2009). Calcium/calmodulin-dependent protein kinase II contributes to cardiac arrhythmogenesis in heart failure. Circulation Heart failure 2, 664-675.

265. Sag, C.M., Wolff, H.A., Neumann, K., Opiela, M.K., Zhang, J., Steuer, F., Sowa, T., Gupta, S., Schirmer, M., Hunlich, M., et al. (2013). Ionizing radiation regulates cardiac Ca handling via increased ROS and activated CaMKII. Basic research in cardiology 108, 385.

266. Sakmann, B., and Neher, E. (1984). Patch clamp techniques for studying ionic channels in excitable membranes. Annual review of physiology 46, 455-472.

267. Salonen, J.T., Nyyssonen, K., Salonen, R., Lakka, H.M., Kaikkonen, J., Porkkala-Sarataho, E., Voutilainen, S., Lakka, T.A., Rissanen, T., Leskinen, L., et al. (2000). Antioxidant Supplementation in Atherosclerosis Prevention (ASAP) study: a randomized trial of the effect of vitamins E and C on 3-year progression of carotid atherosclerosis. Journal of internal medicine 248, 377-386.

268. Sanguinetti, M.C., and Bennett, P.B. (2003). Antiarrhythmic drug target choices and screening. Circ Res 93, 491-499.

269. Santacruz-Toloza, L., Ottolia, M., Nicoll, D.A., and Philipson, K.D. (2000). Functional analysis of a disulfide bond in the cardiac $\mathrm{Na}(+)-\mathrm{Ca}(2+)$ exchanger. J Biol Chem 275, 182-188.

270. Scherer, N.M., and Deamer, D.W. (1986). Oxidative stress impairs the function of sarcoplasmic reticulum by oxidation of sulfhydryl groups in the Ca2+-ATPase. Archives of biochemistry and biophysics 246, 589-601.

271. Scheuer, T. (2011). Regulation of sodium channel activity by phosphorylation. Seminars in cell \& developmental biology 22, 160-165.

272. Schillaci, G., Verdecchia, P., Borgioni, C., Ciucci, A., Zampi, I., Battistelli, M., Gattobigio, R., Sacchi, N., and Porcellati, C. (1996). Association between persistent pressure overload and ventricular arrhythmias in essential hypertension. Hypertension 28, 284-289.

273. Scholten, A., van Veen, T.A., Vos, M.A., and Heck, A.J. (2007). Diversity of cAMP-dependent protein kinase isoforms and their anchoring proteins in mouse ventricular tissue. Journal of proteome research 6, 1705-1717.

274. Schriner, S.E., Linford, N.J., Martin, G.M., Treuting, P., Ogburn, C.E., Emond, M., Coskun, P.E., Ladiges, W., Wolf, N., Van Remmen, H., et al. (2005). Extension of murine life span by overexpression of catalase targeted to mitochondria. Science 308, 1909-1911.

275. Schulman, H., and Greengard, P. (1978). Stimulation of brain membrane protein phosphorylation by calcium and an endogenous heat-stable protein. Nature 271, 478-479.

276. Schunkert, H., Dzau, V.J., Tang, S.S., Hirsch, A.T., Apstein, C.S., and Lorell, B.H. (1990). Increased rat cardiac angiotensin converting enzyme activity and mRNA expression in pressure overload left ventricular hypertrophy. Effects on coronary resistance, contractility, and relaxation. The Journal of clinical investigation 86, 1913-1920. 
277. Schwarzer, M., Osterholt, M., Lunkenbein, A., Schrepper, A., Amorim, P., and Doenst, T. (2014). Mitochondrial reactive oxygen species production and respiratory complex activity in rats with pressure overload-induced heart failure. The Journal of physiology 592, 3767-3782.

278. Schwinger, R.H., Wang, J., Frank, K., Muller-Ehmsen, J., Brixius, K., McDonough, A.A., and Erdmann, E. (1999). Reduced sodium pump alpha1, alpha3, and beta1-isoform protein levels and $\mathrm{Na}+, \mathrm{K}+-$ ATPase activity but unchanged $\mathrm{Na}+\mathrm{Ca} 2+$ exchanger protein levels in human heart failure. Circulation 99, 2105-2112.

279. Schworer, C.M., Colbran, R.J., and Soderling, T.R. (1986). Reversible generation of a Ca2+independent form of $\mathrm{Ca} 2+$ (calmodulin)-dependent protein kinase II by an autophosphorylation mechanism. J Biol Chem 261, 8581-8584.

280. Sei, C.A., Irons, C.E., Sprenkle, A.B., McDonough, P.M., Brown, J.H., and Glembotski, C.C. (1991). The alpha-adrenergic stimulation of atrial natriuretic factor expression in cardiac myocytes requires calcium influx, protein kinase $\mathrm{C}$, and calmodulin-regulated pathways. J Biol Chem 266, 15910-15916.

281. Semb, S.O., Lunde, P.K., Holt, E., Tonnessen, T., Christensen, G., and Sejersted, O.M. (1998). Reduced myocardial $\mathrm{Na}+, \mathrm{K}(+)$-pump capacity in congestive heart failure following myocardial infarction in rats. J Mol Cell Cardiol 30, 1311-1328.

282. Seshiah, P.N., Weber, D.S., Rocic, P., Valppu, L., Taniyama, Y., and Griendling, K.K. (2002). Angiotensin II stimulation of NAD(P)H oxidase activity: upstream mediators. Circ Res 91, 406413.

283. Shao, D., Oka, S., Brady, C.D., Haendeler, J., Eaton, P., and Sadoshima, J. (2012). Redox modification of cell signaling in the cardiovascular system. J Mol Cell Cardiol 52, 550-558.

284. Shattock, M.J., and Matsuura, H. (1993). Measurement of $\mathrm{Na}(+)-\mathrm{K}+$ pump current in isolated rabbit ventricular myocytes using the whole-cell voltage-clamp technique. Inhibition of the pump by oxidant stress. Circ Res 72, 91-101.

285. Shlafer, M., Myers, C.L., and Adkins, S. (1987). Mitochondrial hydrogen peroxide generation and activities of glutathione peroxidase and superoxide dismutase following global ischemia. J Mol Cell Cardiol 19, 1195-1206.

286. Simmerman, H.K., Collins, J.H., Theibert, J.L., Wegener, A.D., and Jones, L.R. (1986). Sequence analysis of phospholamban. Identification of phosphorylation sites and two major structural domains. J Biol Chem 261, 13333-13341.

287. Song, Y.H., Cho, H., Ryu, S.Y., Yoon, J.Y., Park, S.H., Noh, C.I., Lee, S.H., and Ho, W.K. (2010). L-type $\mathrm{Ca}(2+)$ channel facilitation mediated by $\mathrm{H}(2) \mathrm{O}(2)$-induced activation of CaMKII in rat ventricular myocytes. J Mol Cell Cardiol 48, 773-780.

288. Soonpaa, M.H., and Field, L.J. (1998). Survey of studies examining mammalian cardiomyocyte DNA synthesis. Circ Res 83, 15-26.

289. Soriano, F.X., Baxter, P., Murray, L.M., Sporn, M.B., Gillingwater, T.H., and Hardingham, G.E. (2009). Transcriptional regulation of the AP-1 and Nrf2 target gene sulfiredoxin. Molecules and cells $27,279-282$.

290. Sossalla, S., Maurer, U., Schotola, H., Hartmann, N., Didie, M., Zimmermann, W.H., Jacobshagen, C., Wagner, S., and Maier, L.S. (2011). Diastolic dysfunction and arrhythmias caused by overexpression of CaMKIIdelta(C) can be reversed by inhibition of late $\mathrm{Na}(+)$ current. Basic research in cardiology 106, 263-272.

291. Splawski, I., Timothy, K.W., Decher, N., Kumar, P., Sachse, F.B., Beggs, A.H., Sanguinetti, M.C., and Keating, M.T. (2005). Severe arrhythmia disorder caused by cardiac L-type calcium channel mutations. Proceedings of the National Academy of Sciences of the United States of America 102, 8089-8096; discussion 8086-8088.

292. Sprang, S.R., Chen, Z., and Du, X. (2007). Structural basis of effector regulation and signal termination in heterotrimeric Galpha proteins. Advances in protein chemistry 74, 1-65. 
293. Steichen, J.M., Iyer, G.H., Li, S., Saldanha, S.A., Deal, M.S., Woods, V.L., Jr., and Taylor, S.S. (2010). Global consequences of activation loop phosphorylation on protein kinase A. J Biol Chem 285, 3825-3832.

294. Stephens, N.G., Parsons, A., Schofield, P.M., Kelly, F., Cheeseman, K., and Mitchinson, M.J. (1996). Randomised controlled trial of vitamin E in patients with coronary disease: Cambridge Heart Antioxidant Study (CHAOS). Lancet 347, 781-786.

295. Stotz, S.C., Hamid, J., Spaetgens, R.L., Jarvis, S.E., and Zamponi, G.W. (2000). Fast inactivation of voltage-dependent calcium channels. A hinged-lid mechanism? J Biol Chem 275, 24575-24582.

296. Stotz, S.C., and Zamponi, G.W. (2001). Identification of inactivation determinants in the domain IIS6 region of high voltage-activated calcium channels. J Biol Chem 276, 33001-33010.

297. Studer, R., Reinecke, H., Bilger, J., Eschenhagen, T., Bohm, M., Hasenfuss, G., Just, H., Holtz, J., and Drexler, H. (1994). Gene expression of the cardiac $\mathrm{Na}(+)-\mathrm{Ca} 2+$ exchanger in end-stage human heart failure. Circ Res 75, 443-453.

298. Stull, L.B., Leppo, M.K., Szweda, L., Gao, W.D., and Marban, E. (2004). Chronic treatment with allopurinol boosts survival and cardiac contractility in murine postischemic cardiomyopathy. Circ Res 95, 1005-1011.

299. Summers, R.J., Kompa, A., and Roberts, S.J. (1997). Beta-adrenoceptor subtypes and their desensitization mechanisms. Journal of autonomic pharmacology 17, 331-343.

300. Susic, D., Nunez, E., Frohlich, E.D., and Prakash, O. (1996). Angiotensin II increases left ventricular mass without affecting myosin isoform mRNAs. Hypertension 28, 265-268.

301. Sussman, M.A., Lim, H.W., Gude, N., Taigen, T., Olson, E.N., Robbins, J., Colbert, M.C., Gualberto, A., Wieczorek, D.F., and Molkentin, J.D. (1998). Prevention of cardiac hypertrophy in mice by calcineurin inhibition. Science 281, 1690-1693.

302. Swaminathan, P.D., Purohit, A., Soni, S., Voigt, N., Singh, M.V., Glukhov, A.V., Gao, Z., He, B.J., Luczak, E.D., Joiner, M.L., et al. (2011). Oxidized CaMKII causes cardiac sinus node dysfunction in mice. The Journal of clinical investigation 121, 3277-3288.

303. Swynghedauw, B. (1998). [Left ventricular remodeling. A complex biological problem around three simple paradigms]. Bulletin de l'Academie nationale de medecine 182, 665-682; discussion 683.

304. Takac, I., Schroder, K., Zhang, L., Lardy, B., Anilkumar, N., Lambeth, J.D., Shah, A.M., Morel, F., and Brandes, R.P. (2011). The E-loop is involved in hydrogen peroxide formation by the NADPH oxidase Nox4. J Biol Chem 286, 13304-13313.

305. Takimoto, E., and Kass, D.A. (2007). Role of oxidative stress in cardiac hypertrophy and remodeling. Hypertension 49, 241-248.

306. Taylor, S.S., Buechler, J.A., and Yonemoto, W. (1990). cAMP-dependent protein kinase: framework for a diverse family of regulatory enzymes. Annual review of biochemistry 59, 9711005.

307. Terentyev, D., Gyorke, I., Belevych, A.E., Terentyeva, R., Sridhar, A., Nishijima, Y., de Blanco, E.C., Khanna, S., Sen, C.K., Cardounel, A.J., et al. (2008). Redox modification of ryanodine receptors contributes to sarcoplasmic reticulum Ca2+ leak in chronic heart failure. Circ Res 103, 1466-1472.

308. Theeuwes, F., and Yum, S.I. (1976). Principles of the design and operation of generic osmotic pumps for the delivery of semisolid or liquid drug formulations. Annals of biomedical engineering 4, 343-353.

309. Thurmann, P.A., Kenedi, P., Schmidt, A., Harder, S., and Rietbrock, N. (1998). Influence of the angiotensin II antagonist valsartan on left ventricular hypertrophy in patients with essential hypertension. Circulation 98, 2037-2042.

310. Timolati, F., Ott, D., Pentassuglia, L., Giraud, M.N., Perriard, J.C., Suter, T.M., and Zuppinger, C. (2006). Neuregulin-1 beta attenuates doxorubicin-induced alterations of 
excitation-contraction coupling and reduces oxidative stress in adult rat cardiomyocytes. J Mol Cell Cardiol 41, 845-854.

311. Tipton, K.F., Boyce, S., O'Sullivan, J., Davey, G.P., and Healy, J. (2004). Monoamine oxidases: certainties and uncertainties. Current medicinal chemistry 11, 1965-1982.

312. Toischer, K., Rokita, A.G., Unsold, B., Zhu, W., Kararigas, G., Sossalla, S., Reuter, S.P., Becker, A., Teucher, N., Seidler, T., et al. (2010). Differential cardiac remodeling in preload versus afterload. Circulation 122, 993-1003.

313. Tokarska-Schlattner, M., Zaugg, M., Zuppinger, C., Wallimann, T., and Schlattner, U. (2006). New insights into doxorubicin-induced cardiotoxicity: the critical role of cellular energetics. J Mol Cell Cardiol 41, 389-405.

314. Toyofuku, T., Curotto Kurzydlowski, K., Narayanan, N., and MacLennan, D.H. (1994). Identification of Ser38 as the site in cardiac sarcoplasmic reticulum $\mathrm{Ca}(2+)$-ATPase that is phosphorylated by Ca2+/calmodulin-dependent protein kinase. J Biol Chem 269, 26492-26496.

315. Tripathy, A., and Meissner, G. (1996). Sarcoplasmic reticulum lumenal Ca2+ has access to cytosolic activation and inactivation sites of skeletal muscle Ca2+ release channel. Biophysical journal 70, 2600-2615.

316. Tsutsui, H., Kinugawa, S., and Matsushima, S. (2011). Oxidative stress and heart failure. Am J Physiol Heart Circ Physiol 301, H2181-2190.

317. Ungerer, M., Bohm, M., Elce, J.S., Erdmann, E., and Lohse, M.J. (1993). Altered expression of beta-adrenergic receptor kinase and beta 1-adrenergic receptors in the failing human heart. Circulation 87, 454-463.

318. Ushio-Fukai, M. (2006). Redox signaling in angiogenesis: role of NADPH oxidase. Cardiovasc Res 71, 226-235.

319. Vinson, G.P., Ho, M.M., and Puddefoot, J.R. (1995). The distribution of angiotensin II type 1 receptors, and the tissue renin-angiotensin systems. Molecular medicine today 1, 35-39.

320. Virdis, A., Neves, M.F., Amiri, F., Touyz, R.M., and Schiffrin, E.L. (2004). Role of NAD(P)H oxidase on vascular alterations in angiotensin II-infused mice. Journal of hypertension 22, 535542.

321. Virtamo, J., Rapola, J.M., Ripatti, S., Heinonen, O.P., Taylor, P.R., Albanes, D., and Huttunen, J.K. (1998). Effect of vitamin $E$ and beta carotene on the incidence of primary nonfatal myocardial infarction and fatal coronary heart disease. Archives of internal medicine 158, 668-675.

322. Wagner, S., Dantz, C., Flebbe, H., Azizian, A., Sag, C.M., Engels, S., Mollencamp, J., Dybkova, N., Islam, T., Shah, A.M., et al. (2014). NADPH oxidase 2 mediates angiotensin II-dependent cellular arrhythmias via PKA and CaMKII. J Mol Cell Cardiol 75, 206-215.

323. Wagner, S., Dybkova, N., Rasenack, E.C., Jacobshagen, C., Fabritz, L., Kirchhof, P., Maier, S.K., Zhang, T., Hasenfuss, G., Brown, J.H., et al. (2006). Ca2+/calmodulin-dependent protein kinase II regulates cardiac Na+ channels. The Journal of clinical investigation 116, 3127-3138.

324. Wagner, S., Maier, L.S., and Bers, D.M. (2015). Role of sodium and calcium dysregulation in tachyarrhythmias in sudden cardiac death. Circ Res 116, 1956-1970.

325. Wagner, S., Rokita, A.G., Anderson, M.E., and Maier, L.S. (2013). Redox regulation of sodium and calcium handling. Antioxidants \& redox signaling 18, 1063-1077.

326. Wagner, S., Ruff, H.M., Weber, S.L., Bellmann, S., Sowa, T., Schulte, T., Anderson, M.E., Grandi, E., Bers, D.M., Backs, J., et al. (2011). Reactive oxygen species-activated Ca/calmodulin kinase IIdelta is required for late I(Na) augmentation leading to cellular $\mathrm{Na}$ and Ca overload. Circ Res 108, 555-565.

327. Wang, J., Liu, X., Sentex, E., Takeda, N., and Dhalla, N.S. (2003). Increased expression of protein kinase $\mathrm{C}$ isoforms in heart failure due to myocardial infarction. Am J Physiol Heart Circ Physiol 284, H2277-2287. 
328. Wang, R., Wang, Y., Lin, W.K., Zhang, Y., Liu, W., Huang, K., Terrar, D.A., Solaro, R.J., Wang, $\mathrm{X} ., \mathrm{Ke}, \mathrm{Y}$., et al. (2014). Inhibition of angiotensin II-induced cardiac hypertrophy and associated ventricular arrhythmias by a p21 activated kinase 1 bioactive peptide. PloS one 9, e101974.

329. Wang, W., Zhu, W., Wang, S., Yang, D., Crow, M.T., Xiao, R.P., and Cheng, H. (2004). Sustained beta1-adrenergic stimulation modulates cardiac contractility by $\mathrm{Ca}+/$ calmodulin kinase signaling pathway. Circ Res 95, 798-806.

330. Waters, D.D., Alderman, E.L., Hsia, J., Howard, B.V., Cobb, F.R., Rogers, W.J., Ouyang, P., Thompson, P., Tardif, J.C., Higginson, L., et al. (2002). Effects of hormone replacement therapy and antioxidant vitamin supplements on coronary atherosclerosis in postmenopausal women: a randomized controlled trial. Jama 288, 2432-2440.

331. Weber, K.T., Brilla, C.G., and Janicki, J.S. (1993). Myocardial fibrosis: functional significance and regulatory factors. Cardiovasc Res 27, 341-348.

332. Wehrens, X.H., Lehnart, S.E., Reiken, S.R., and Marks, A.R. (2004). Ca2+/calmodulindependent protein kinase II phosphorylation regulates the cardiac ryanodine receptor. Circ Res 94, e61-70.

333. Wen, H., Gwathmey, J.K., and Xie, L.H. (2012). Oxidative stress-mediated effects of angiotensin II in the cardiovascular system. World journal of hypertension 2, 34-44.

334. Werns, S.W., Shea, M.J., Driscoll, E.M., Cohen, C., Abrams, G.D., Pitt, B., and Lucchesi, B.R. (1985). The independent effects of oxygen radical scavengers on canine infarct size. Reduction by superoxide dismutase but not catalase. Circ Res 56, 895-898.

335. Wood, Z.A., Schroder, E., Robin Harris, J., and Poole, L.B. (2003). Structure, mechanism and regulation of peroxiredoxins. Trends in biochemical sciences 28, 32-40.

336. Xiao, L., Pimentel, D.R., Wang, J., Singh, K., Colucci, W.S., and Sawyer, D.B. (2002). Role of reactive oxygen species and $\mathrm{NAD}(\mathrm{P}) \mathrm{H}$ oxidase in alpha(1)-adrenoceptor signaling in adult rat cardiac myocytes. American journal of physiology Cell physiology 282, C926-934.

337. Xie, L.H., Chen, F., Karagueuzian, H.S., and Weiss, J.N. (2009). Oxidative-stress-induced afterdepolarizations and calmodulin kinase II signaling. Circ Res 104, 79-86.

338. Xie, Z.J., Wang, Y.H., Askari, A., Huang, W.H., Klaunig, J.E., and Askari, A. (1990). Studies on the specificity of the effects of oxygen metabolites on cardiac sodium pump. J Mol Cell Cardiol 22, 911-920.

339. Xu, K.Y., Zweier, J.L., and Becker, L.C. (1997). Hydroxyl radical inhibits sarcoplasmic reticulum $\mathrm{Ca}(2+)$-ATPase function by direct attack on the ATP binding site. Circ Res 80, 76-81.

340. Xu, L., Eu, J.P., Meissner, G., and Stamler, J.S. (1998a). Activation of the cardiac calcium release channel (ryanodine receptor) by poly-S-nitrosylation. Science 279, 234-237.

341. Xu, L., Tripathy, A., Pasek, D.A., and Meissner, G. (1998b). Potential for pharmacology of ryanodine receptor/calcium release channels. Annals of the New York Academy of Sciences 853, 130-148.

342. Yamaguchi, O., Higuchi, Y., Hirotani, S., Kashiwase, K., Nakayama, H., Hikoso, S., Takeda, T., Watanabe, T., Asahi, M., Taniike, M., et al. (2003). Targeted deletion of apoptosis signalregulating kinase 1 attenuates left ventricular remodeling. Proceedings of the National Academy of Sciences of the United States of America 100, 15883-15888.

343. Yang, L., Liu, G., Zakharov, S.I., Morrow, J.P., Rybin, V.O., Steinberg, S.F., and Marx, S.O. (2005). Ser1928 is a common site for Cav1.2 phosphorylation by protein kinase C isoforms. J Biol Chem 280, 207-214.

344. Yano, M., Okuda, S., Oda, T., Tokuhisa, T., Tateishi, H., Mochizuki, M., Noma, T., Doi, M., Kobayashi, S., Yamamoto, T., et al. (2005). Correction of defective interdomain interaction within ryanodine receptor by antioxidant is a new therapeutic strategy against heart failure. Circulation $112,3633-3643$. 
345. Yasunari, K., Maeda, K., Nakamura, M., and Yoshikawa, J. (2002). Pressure promotes angiotensin II--mediated migration of human coronary smooth muscle cells through increase in oxidative stress. Hypertension 39, 433-437.

346. Youn, J.Y., Zhang, J., Zhang, Y., Chen, H., Liu, D., Ping, P., Weiss, J.N., and Cai, H. (2013). Oxidative stress in atrial fibrillation: an emerging role of NADPH oxidase. J Mol Cell Cardiol 62, 72-79.

347. Yusuf, S., Dagenais, G., Pogue, J., Bosch, J., and Sleight, P. (2000). Vitamin E supplementation and cardiovascular events in high-risk patients. The Heart Outcomes Prevention Evaluation Study Investigators. The New England journal of medicine 342, 154-160.

348. Zable, A.C., Favero, T.G., and Abramson, J.J. (1997). Glutathione modulates ryanodine receptor from skeletal muscle sarcoplasmic reticulum. Evidence for redox regulation of the $\mathrm{Ca} 2+$ release mechanism. J Biol Chem 272, 7069-7077.

349. Zakhary, D.R., Moravec, C.S., and Bond, M. (2000). Regulation of PKA binding to AKAPs in the heart: alterations in human heart failure. Circulation 101, 1459-1464.

350. Zakhary, D.R., Moravec, C.S., Stewart, R.W., and Bond, M. (1999). Protein kinase A (PKA)dependent troponin-I phosphorylation and PKA regulatory subunits are decreased in human dilated cardiomyopathy. Circulation 99, 505-510.

351. Zankov, D.P., Omatsu-Kanbe, M., Isono, T., Toyoda, F., Ding, W.G., Matsuura, H., and Horie, M. (2006). Angiotensin II potentiates the slow component of delayed rectifier K+ current via the AT1 receptor in guinea pig atrial myocytes. Circulation 113, 1278-1286.

352. Zhang, G.X., Lu, X.M., Kimura, S., and Nishiyama, A. (2007). Role of mitochondria in angiotensin II-induced reactive oxygen species and mitogen-activated protein kinase activation. Cardiovasc Res 76, 204-212.

353. Zhang, J., Youn, J.Y., Kim, A.Y., Ramirez, R.J., Gao, L., Ngo, D., Chen, P., Scovotti, J., Mahajan, A., and Cai, H. (2012). NOX4-Dependent Hydrogen Peroxide Overproduction in Human Atrial Fibrillation and HL-1 Atrial Cells: Relationship to Hypertension. Frontiers in physiology 3, 140.

354. Zhang, J.F., Ellinor, P.T., Aldrich, R.W., and Tsien, R.W. (1994). Molecular determinants of voltage-dependent inactivation in calcium channels. Nature 372, 97-100.

355. Zhang, M., Perino, A., Ghigo, A., Hirsch, E., and Shah, A.M. (2013). NADPH oxidases in heart failure: poachers or gamekeepers? Antioxidants \& redox signaling 18, 1024-1041.

356. Zhang, T., Johnson, E.N., Gu, Y., Morissette, M.R., Sah, V.P., Gigena, M.S., Belke, D.D., Dillmann, W.H., Rogers, T.B., Schulman, H., et al. (2002). The cardiac-specific nuclear delta(B) isoform of $\mathrm{Ca} 2+/$ calmodulin-dependent protein kinase II induces hypertrophy and dilated cardiomyopathy associated with increased protein phosphatase 2A activity. J Biol Chem 277, 1261-1267.

357. Zhang, T., Maier, L.S., Dalton, N.D., Miyamoto, S., Ross, J., Jr., Bers, D.M., and Brown, J.H. (2003). The deltaC isoform of CaMKII is activated in cardiac hypertrophy and induces dilated cardiomyopathy and heart failure. Circ Res 92, 912-919.

358. Zhang, X., Min, X., Li, C., Benjamin, I.J., Qian, B., Zhang, X., Ding, Z., Gao, X., Yao, Y., Ma, Y., et al. (2010). Involvement of reductive stress in the cardiomyopathy in transgenic mice with cardiac-specific overexpression of heat shock protein 27. Hypertension 55, 1412-1417.

359. Zhao, Z., Fefelova, N., Shanmugam, M., Bishara, P., Babu, G.J., and Xie, L.H. (2011). Angiotensin II induces afterdepolarizations via reactive oxygen species and calmodulin kinase II signaling. J Mol Cell Cardiol 50, 128-136.

360. Zhao, Z., Xie, Y., Wen, H., Xiao, D., Allen, C., Fefelova, N., Dun, W., Boyden, P.A., Qu, Z., and Xie, L.H. (2012). Role of the transient outward potassium current in the genesis of early afterdepolarizations in cardiac cells. Cardiovasc Res 95, 308-316.

361. Zhu, W., Zou, Y., Shiojima, I., Kudoh, S., Aikawa, R., Hayashi, D., Mizukami, M., Toko, H., Shibasaki, F., Yazaki, Y., et al. (2000). Ca2+/calmodulin-dependent kinase II and calcineurin play critical roles in endothelin-1-induced cardiomyocyte hypertrophy. J Biol Chem 275, 1523915245. 
362. Zhu, W.Z., Wang, S.Q., Chakir, K., Yang, D., Zhang, T., Brown, J.H., Devic, E., Kobilka, B.K., Cheng, H., and Xiao, R.P. (2003). Linkage of beta1-adrenergic stimulation to apoptotic heart cell death through protein kinase A-independent activation of $\mathrm{Ca} 2+/$ calmodulin kinase II. The Journal of clinical investigation 111, 617-625.

363. Zorov, D.B., Filburn, C.R., Klotz, L.O., Zweier, J.L., and Sollott, S.J. (2000). Reactive oxygen species (ROS)-induced ROS release: a new phenomenon accompanying induction of the mitochondrial permeability transition in cardiac myocytes. The Journal of experimental medicine $192,1001-1014$.

364. Zorov, D.B., Juhaszova, M., and Sollott, S.J. (2006). Mitochondrial ROS-induced ROS release: an update and review. Biochimica et biophysica acta 1757, 509-517.

365. Zweier, J.L., and Talukder, M.A. (2006). The role of oxidants and free radicals in reperfusion injury. Cardiovasc Res 70, 181-190. 


\section{Currúcutum vitae}




\section{M.M.TOWHIDUL ISLAM}

Kiesseestrasse 17,

37083 Göttingen, Germany

Mobile: +49015163406834

E-mail : mmtowhidul.islam@stud.uni-goettingen.de \&

towhidbmb@du.ac.bd

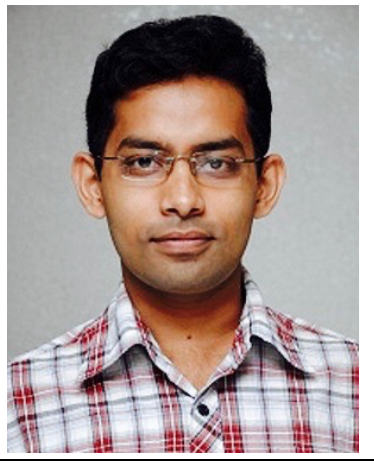

Present status Lecturer, Department of Biochemistry and Molecular biology (from 01.03.12 to present)

\section{Education}

Degree

\begin{tabular}{lccc}
\cline { 2 - 4 } & Institution & Year & Result \\
\hline M.S in & Dhaka & 2009 & 1 st class 2 \\
Biochemistry and & University & & $76.67 \%$ \\
Molecular biology & & &
\end{tabular}

\begin{tabular}{|c|c|c|c|}
\hline B.Sc in & Dhaka & 2008 & 1 st class $1^{\text {st }}$ \\
\hline Biochemistry and & University & & $75.44 \%$ \\
\hline
\end{tabular}

\begin{tabular}{lccc}
\hline $\begin{array}{l}\text { Higher Secondary } \\
\text { Certificate (HSC) }\end{array}$ & $\begin{array}{c}\text { Sylhet cadet } \\
\text { College }\end{array}$ & 2004 & $\begin{array}{c}\mathbf{5 . 0 0} \\
\text { (out of 5.00) } \\
\text { with } 4^{\text {th }} \text { subject }\end{array}$ \\
\hline $\begin{array}{l}\text { Secondary School } \\
\text { Certificate (SSC) }\end{array}$ & $\begin{array}{c}\text { Sylhet cadet } \\
\text { College }\end{array}$ & 2002 & $\begin{array}{c}\mathbf{5 . 0 0} \\
\text { (out of 5.00) } \\
\text { with } 4^{\text {th }} \text { subject }\end{array}$
\end{tabular}

\section{Working}

Experiences
- Research Fellow at ICDDR,B (International Centre for Diarrhoeal Disease Research, Bangladesh) from 2009 till 2011

- Research Officer at ICDDR,B (International Centre for Diarrhoeal Disease Research, Bangladesh) from 2011 till 2012

- Teacher at Department of Biochemistry and Molecular Biology in University of Dhaka from 2012 till now

- PhD fellow in Department of Cardiology and Pneumology, University Medical Center, George August University Göttingen from 2013 till now 
Identifying Antibiotics posing potential Health Risk: Microbial Resistance Scenario in Bangladesh. (2015) Atai Rabby, Rasel Al Mahmud, Towhidul MM Islam, Yearul Kabir, Md. Rakibul Islam. International Journal of Medical and Health Sciences,

4, 90-97.

NADPH oxidase 2 mediates angiotensin II-dependent cellular arrhythmias via PKA and CaMKII. (2014) Stefan Wagner, Christian Dantz, Hannah Flebbe, Azadeh Azizian, Can Martin Sag, Susanne Engels, Johanna Möllencamp, Nataliya Dybkova, Towhidul Islam, Ajay M. Shah, Lars S. Maier. Journal of Molecular and Cellular Cardiology, 75, 206-215.

Th1 and Th17 Responses to Helicobacter pylori in Bangladeshi Infants, Children and Adults. (2014) Taufiqur R. Bhuiyan, M M. Towhidul Islam, Taher Uddin, Mohiul I. Chowdhury, Anders Janzon, Jenni Adamsson, Samuel B. Lundin, Firdausi Qadri, Anna Lundgren. Plos one, 9, 1-9.

Food Functionality of Popular and Commonly Consumed Indigenous Vegetables and Fruits from Bangladesh. (2013) M. M. Towhidul Islam, Arnab Talukder, Taibur Rahman, Jahid M. M. Islam, Shahdat Hossain, Hossain Uddin Shekhar. Food and Nutrition Sciences, 4, 741-747.

Identification of Genes of CRISPR Associated Proteins CAS in the Genomes of Enteric Microorganisms. (2013) Md. Atai Rabby, Towhidul MM Islam, Md. Hasibur Rahman, Md. Rakibul Islam. Biomirror, 4, 13-16.

Oxidative stress and human health. (2012) Taibur Rahman, Md. Ismail Hosen, M M Towhidul Islam, Hossain Uddin Shekhar. Advances in Bioscience and Biotechnology, 3, 997-1019.

Book Chapter: Impact of Oxidative Stress on Human Health. (2014) M. M. Towhidul Islam, Hossain Uddin Shekhar. Free Radicals in Human Health and Disease, Springer Link, 59-73.

CASK is an important regulator of cardiac excitation-contraction coupling. J. Mustroph, S. Gupta, A. Dietz, F. Bähr, T. Islam, A. El-Armouche, L.S. Maier, S. Wagner. (manuscript under preparation)

Sleep-disordered breathing is associated with increased CaMKII-dependent SR $\mathrm{Ca}^{2+}$ leak in human Atrial Cardiomyocytes. M.A. Drzymalski, S. Ripfel, S. Meindl, A. Biedermann, M. Durczok, T. Islam, S. Katz, M. Mendl, T. Weizenegger, B. Flörchinger, D. Camboni, S. Wittmann, J. Backs, C. Schmid, L.S. Maier, M. Arzt, S. Wagner. (manuscript under preparation) 
Relationship between postmenopausal obesity and CaMKII via p38MAP kinase pathway in the atrium. T. Tsuneda, S. Neef, T. Islam, L. S. Maier (manuscript under preparation)

\section{Abstract presented as posters in International meetings:}

- Disruption of oxidative PKA activation contributes to ventricular remodeling upon increased afterload. T. Islam, C. Dantz, H. Daniel, J. Moellencamp, A. ElArmouche, P. Eaton, LS. Maier, S. Wagner.

European Society of Cardiology Congress 2014, Barcelona, Spain. 30.08.1403.09.14.

- Disruption of oxidative PKA activation prevents Ang II-dependent hypertrophy. T. Islam, C. Dantz, H. Daniel, J. Moellencamp, A. El-Armouche, P. Eaton, LS. Maier, S. Wagner.

Heart Rhythm Congress 2015, Boston, Massachusetts, USA. Wednesday, May 13, 2015 - Saturday, May 16, 2015

- Th1 and Th17 responses to Helicobacter pylori in Bangladeshi children and adults. Taufiqur Rahman Bhuiyan, Towhid Islam, Samuel B Lundin, Anna Lundgren, Firdausi Qadri.

$15^{\text {th }}$ International Congress of Mucosal Immunology (ICMI 2011), Paris, France, from 5 to 9 July 2011.

\section{Abstract presented in International meetings:}

Regulation of Cardiac Ca channel gating by reactive oxygen species

- Molecular Medicine Retreat, Braunlage, Germany. 11.09.14-12.09.14

- International Research Training Group Retreat, Uslar, Germany. 21.10.1322.10 .13

- Training on Animal Use and care, modules include Guidelines, Legislation and Regulations, Ethics in Animal, Experimentation, Three Rs of Humane Animal Experimentation, Research Issues, Basic Animal Care etc

- Training on Good Scientific Practice (GSP), modules include How to avoid plagiarism, Publishing Papers in the Natural Sciences, Research Data Management etc

- Training on FRET microscopy, modules include Principles of Fluorescence and Fluorescence Microscopy, Image processing and presentation, Contrast enhancement in transmitted light etc.

- Training on Electrophysiology and Patch Clamp, modules include Principles and mechanisms, Intracellular recording, Extracellular recording, patch-clamp technique, Other Optical electrophysiological techniques etc.

- Training on Mass Spectrophotometry, modules include Introduction to different parts of a mass spectrometer, How to create ions for MS, Mass selection, Detectors and how they work etc.

- Training on Mouse Echocardiography, modules include What is Echocardiography, How it can be used for Rodents, why it is important to 
detect Heart diseases etc

- Training on Bio-safety that includes safe laboratory design, general laboratory safety, chemical safety and waste management.

- Training on Good Clinical Practice (GCP) and topics covering were GCP overview, investigator responsibilities, IRB/IEC, study product accountability, informed consent, adverse events/serious adverse events, essential documents, source documentation, quality management, unanticipated problems.

\section{Computer Skills}

- Programming Language: C.

- Adobe Photoshop, Leica QWin, Sigma-Stat, Sigma-Plot, CellQuest Pro, FlowJo, Graph pad, Image J, Vevo lab, LabChart

- Operating Systems: Windows 7, Mac OX, Ubuntu

- Expert in Office Applications like Microsoft Word, Microsoft Excel and Microsoft Power point.

\section{Academic} Achievements
- Achieved "Deans Award" given by the Dean of Faculty of Biological Sciences, University of Dhaka for excellent performance in B.Sc Honors level.

- Awarded by Nippon Foundation, Japan and Bangladesh Scholarship Council for excellent academic results during B.Sc Honors.

- Awarded by Dhaka Bank limited for excellent academic results in B.Sc Honors final exam.

- Combinedly awarded as "Best Student-2008" by Scholar Bangladesh and Ethics Club Bangladesh for outstanding performance in B.Sc Honors level.

- Awarded "Dr. Motiur Rahman Memorial Trust Scholarship" by Department of Biochemistry and Molecular Biology, University of Dhaka for excellent academic results and extracurricular activities.

- Awarded scholarships for achievement of good grades in both S.S.C and H.S.C examinations by Sylhet Education Board and Sylhet Cadet College authority in 2002 and 2004 respectively.

- Received Primary School Scholarship in first grade on the basis of good results in 1996.

\section{Membership}

- Former Publication Editor of Science Magazine named "Biowatch"

- Member of Graduate Biochemist Association (GBA)

- Member of American Society for Microbiology (ASM)

- Member of the Journal Club of ICDDR,B

- Member of DZHK (young scientist, German Centre for Cardiovascular Research)

- Member of European Society of Cardiology

- Member of Heart Rhythm Society, USA 
- Name: M.M. Towhidul Islam

- Present Address: Kiesseestrasse 17, 37083 Göttingen, Germany

- Permanent Address: 464, South Monipur, Mirpur, Dhaka.

- Date of Birth: November 15, 1986.

- Religion: Islam.

- Nationality: Bangladeshi.

- Marital status: Married.

I, hereby declare that the above information is true to the best of my knowledge and belief. 